\title{
1997 Report on \\ Hanford Site Land \\ Disposal Restrictions \\ for Mixed Waste
}

D. G. Black

Date Published

April 1997

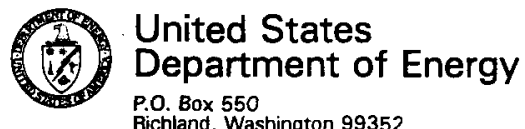

Richland, Washington 99352 
DOE/RL-97-14

This page intentionally left blank. 
LEGAL DISCLAME

This repoit was propaicd an an account of work ppongorad by

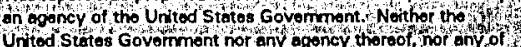
thot onployeos, nor any of thoir contractors, subcontractors

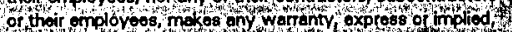

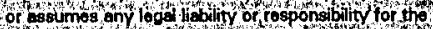
acouracy completenos, of any third party uso or the yesults of auch use of any information epperotus, produch or process

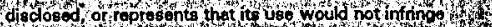

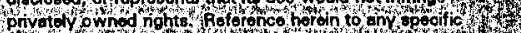

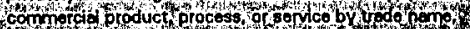

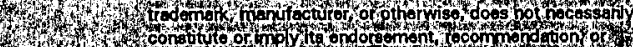

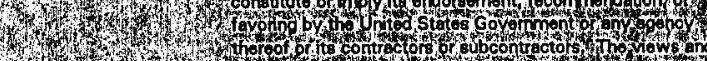

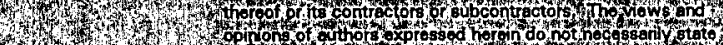

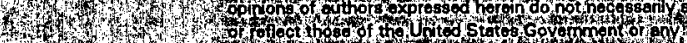

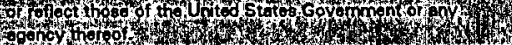

1
3

Hot

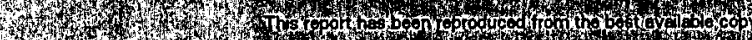

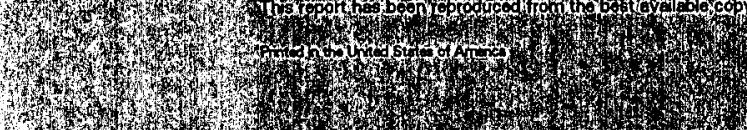

\begin{aligned} \hline \\ \hline\end{aligned}

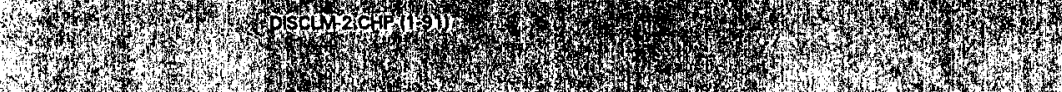
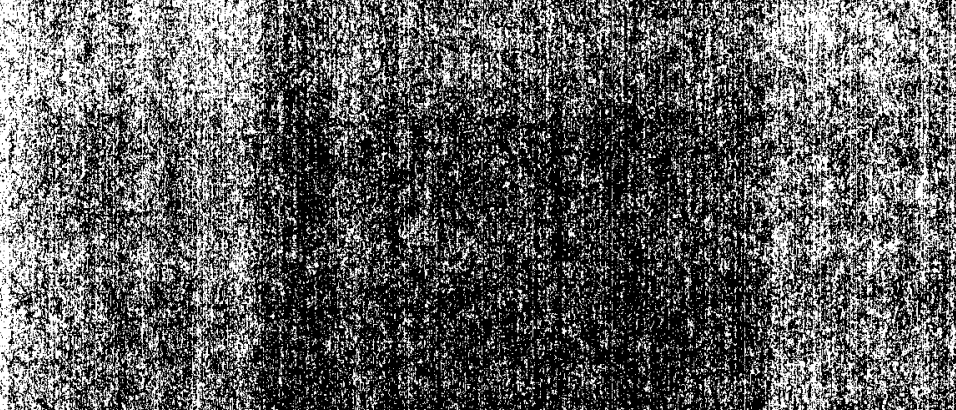

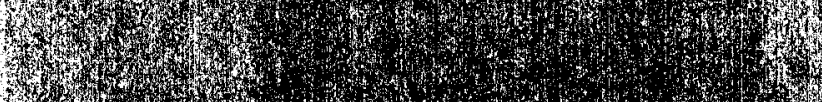

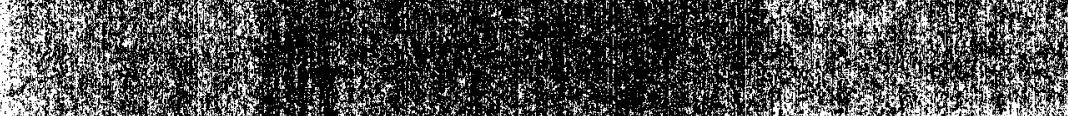

*4.

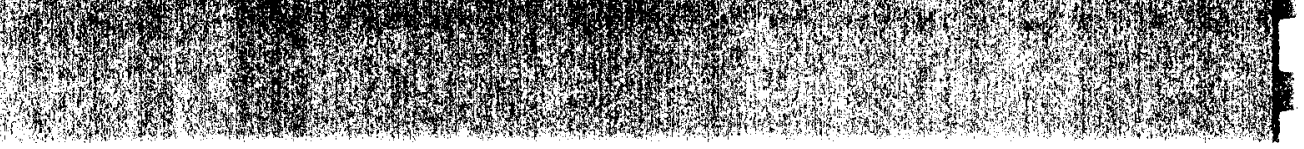


T! i... IILANK 


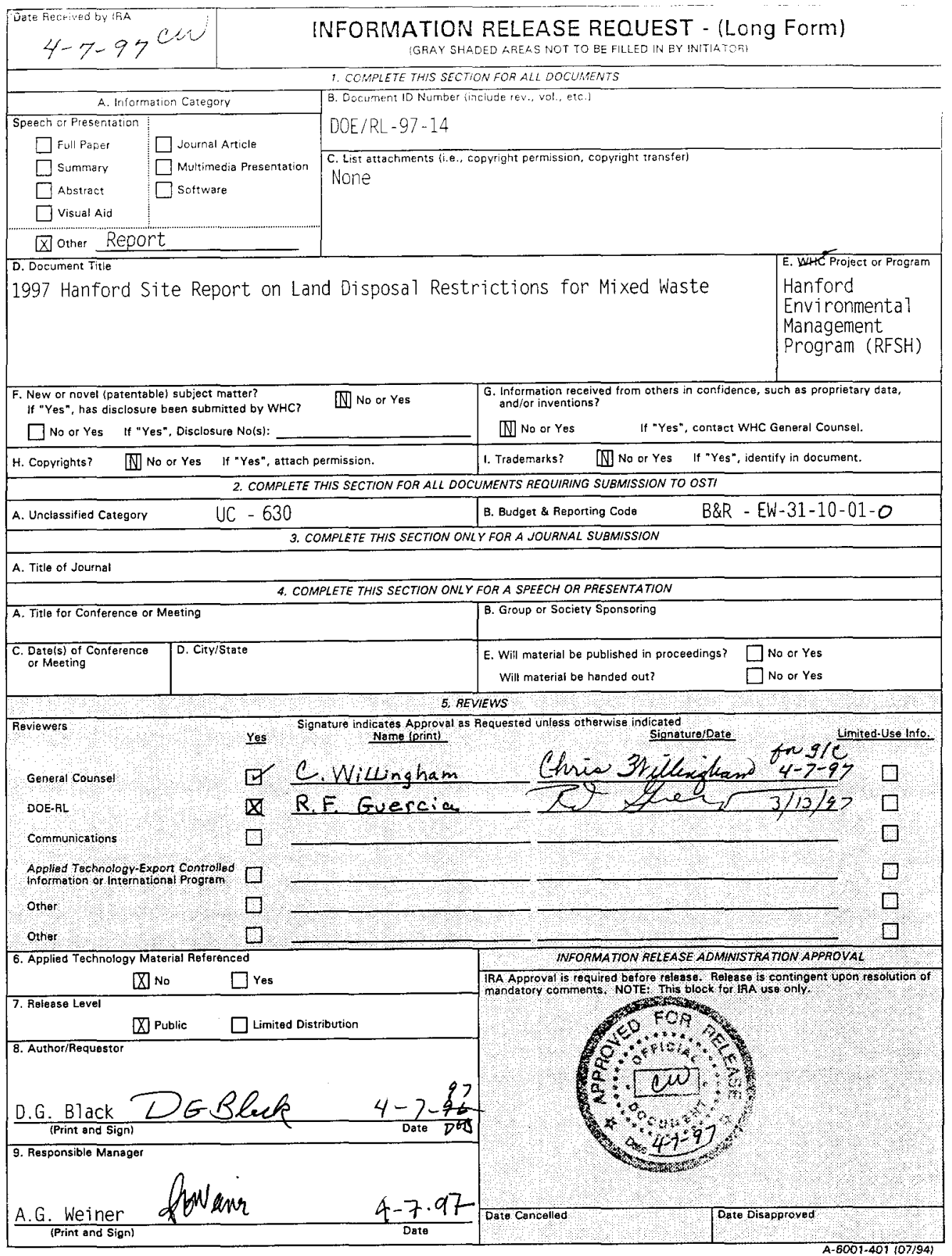


THIS IUITENTONALGY 1... '' BLANK

\section{1}




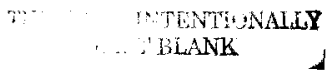




\section{EXECUTIVE SUMMARY}

This report was submitted to meet the requirements of Hanford Federal Facility I Agreement and Consent Order ${ }^{1}$ Milestone M-26-01G. This milestone requires the preparation of an annual report that covers characterization, treatment, storage, minimization, and other aspects of land-disposal-restricted mixed waste management at the Hanford Site.

The U.S. Department of Energy, its predecessors, and contractors at the Hanford Site were involved in the production and purification of nuclear defense materials from the early 1940 s to the late 1980s. These production activities have generated large quantities of liquid and solid radioactive mixed waste. This waste is subject to regulation under authority of both the Resource Conservation and Recovery Act of $1976^{2}$ and the Atomic Energy Act of $1954^{3}$ This report covers mixed waste only.

The Washington State Department of Ecology, U.S. Environmental Protection Agency, and U.S. Department of Energy have entered into an agreement, the Hanford Federal Facility Agreement and Consent Order ${ }^{1}$ (commonly referred to as the Tri-Party Agreement) (Ecology et al. 1989) to bring the Hanford Site operations into compliance with dangerous waste regulations. The Tri-Party Agreement required development of the original land disposal restrictions (LDRs) plan and its annual updates to comply with LDR requirements for radioactive mixed waste. This report is the seventh update of the plan first issued in 1990.

Tri-Party Agreement negotiations completed and approved in 1996 added new milestones to the schedule. These milestones are related to the acquisition and modification of facilities for the storage, treatment, and disposal of tank waste, low-level waste, transuranic waste, and other categories of waste. Changes related to these new milestones are incorporated into this report.

The Tri-Party Agreement requires, and the baseline plan and annual update reports provide, the information that follows.

- Waste Characterization Information--Provides information regarding the characterizing of each LDR mixed waste. The sampling and analysis methods and protocols, past characterization results, and, where available, a schedule for providing the characterization information are discussed.

- Storage Data-Identifies and describes the mixed waste at the Hanford Site, including the following: the Resource Conservation and Recovery Act of 1976 dangerous waste code(s), generator process knowledge necessary to identify the

${ }^{1}$ Ecology, EPA, and DOE, 1989, Hanford Federal Facility Agreement and Consent Order, Vol. 1 and 2, as updated by the sixth amendment dated February 1996, Washington State Department of Ecology, U.S. Environmental Protection Agency, and U.S. Department of Energy, Olympia, Washington.

${ }^{2}$ Resource Conservation and Recovery Act of 1976, as amended, 42 U.S.C. 6901, et seq.

${ }^{9}$ Atomic Energy Act of 1954, as amended, 42 U.S.C. 2011. 
waste and make LDR determinations, quantities stored, generation rates, location and method of storage, an assessment of storage-unit compliance status, storage capacity, and the bases and assumptions used in making the estimates.

- Treatment Information-Identifies the current treatment processes, plans, and schedules for developing treatment technologies that meet LDR treatment standards. Also includes discussions of treatment alternatives and accelerated treatment.

- Waste Reduction Information-Identifies methods for reducing the generation of land-disposal-restricted waste. Includes treatment methods and process changes made or planned to reduce the generation of LDR waste, methods to minimize the volume of LDR waste, and methods to minimize the toxicity of newly generated waste.

- Schedule - Provides schedules depicting the events necessary to achieve compliance with LDR requirements, including planned or completed variances, treatment equivalencies, or time extensions necessary to achieve LDRs compliance.

- Progress-Identifies progress made in achieving compliance since the previous LDRs report.

The Hanford Site waste primarily resulted from defense materials production. Usable defense materials were separated from fission products waste through precipitation and solvent extraction processes. Large quantities of liquid waste resulted from these separation processes and were stored in underground single-shell tanks (SST) and double-shell tanks (DST)

Additional waste volumes resulted from the following:

- Nuclear fuel fabrication activities

- Process laboratory activities

- Equipment and structure cleaning and decontamination

- Process and storage unit closure

- Research and development activities, such as Fast Flux Test Facility operation.

Total projected annual generation rates for the streams covered in this report, after waste reduction, range from a low of 9,720 cubic meters in 2001 to a high of 33,021 cubic meters in 1998 .

The waste addressed in this report includes mixed waste (i.e., hazardous waste that contains radionuclides) designated as characteristic dangerous waste; designated as toxic or persistent by the Washington State criteria; and listed waste because it contains small amounts of spent solvents and discarded pure chemical products. The waste consists of liquid, sludges, hard crystalline material (salt cake), and materials such as contaminated equipment, paper, and rags. Much is already known about the waste characteristics from process knowledge and sampling and analysis programs. Action schedules have been developed to further characterize the waste. 
The waste currently is stored in underground SSTs and DSTs, in containers placed in storage units such as the Central Waste Complex, caissons, and retrievable storage units. A surface impoundment, the Liquid Effluent Retention Facility, has been constructed to store large quantities of waste water that contain radionuclide concentrations low enough to allow surface storage. The waste is removed from these storage units, treated to meet LDR standards, and sent to final disposal in accordance with schedules established in Tri-Party Agreement milestones.

Total storage capacity for LDR waste at Hanford Site facilities is approximately 579,700 cubic meters. About 373,000 cubic meters of this capacity are in units such as SSTs that no longer receive waste. Approximately 226,700 cubic meters of waste are currentiy in storage. The DSTs are expected to be full by 1998 under the current planning baseline. To alleviate the space shortages, DST contents will be concentrated through the 242-A Evaporator. This has allowed DOE to indefinitely postpone the construction of new DSTs. The 242-A Evaporator processed 4,800 cubic meters of waste into the Liquid Effluent Retention Facility basins (included in total storage capacity) in 1996. The storage space currently available at the Central Waste Complex was anticipated to be filled in 1996; however, with the addition of three storage buildings coming into service in 1997, this has been delayed until at least 2001. Also, a shift in programmatic requirements has postponed reaching the forecast waste volume. (Volumes have not been reduced for outyears. Additional buildings will be constructed as required to store waste generated in the future.)

The waste treatment processes for these wastes include the current treatment processes to reduce corrosion of storage tanks and planned treatment processes to reduce waste toxicity and immobilize waste constituents (DSTs). Current waste treatment consists of adjusting $\mathrm{pH}$ and adding corrosion inhibitors (DSTs), and using sorbents and solidifying agents (requisites placed on generators before receiving the waste into the Central Waste Complex). Surface decontamination (i.e., washing, grit blasting, dry ice pellet blasting) is performed at T Plant facilities. Planned waste treatment processes include developing neutralization and toxic constituent destruction processes; developing waste separation, pretreatment, and stabilization processes (commercial facilities); and separating tank waste (pretreatment) into low-activity waste and high-level waste fractions, both of which will be vitrified. The low-level fraction will be disposed of on site. The high-level fraction will be sent to an offsite geologic repository for disposal.

The Hanford Site developed a sitewide waste minimization plan that sets minimization goals and establishes processes for measuring progress toward these goals. Each plant or process has a program in place to implement the sitewide goals.

The continued storage of land disposal restricted wastes until sufficient treatment and disposal capacity is available was negotiated as part of the Tri-Party Agreement. Schedules to implement the dangerous waste management compliance activities until treatment capacity is available are described in the Tri-Party Agreement. Any newly identified compliance actions will be scheduled in accordance with procedures established in the agreement. 
The Hanford Facility is the only DOE site with a preexisting agreement (Tri-Party Agreement) that meets the legal requirements specified under the Federal Facilities Compliance I Act. Having this agreement exempts the Hanford Site from having to develop a site treatment

plan, although other activities under the Act were still required. This exemption is supported by written exemptions from the Washington State Department of Ecology and the U.S.

Environmental Protection Agency. Both agencies determined that this Hanford Site Land Disposal Restrictions Report for Mixed Waste, required by the Tri-Party Agreement, meets the requirements of a site treatment plan. 


\section{ACKNOWLEDGMENTS}

The efforts of many people across the Hanford Site were instrumental in preparing and issuing this report. Direct major support was provided by the following:

Report Lead Author and Coordinator:

D. G. Black Rust Federal Services Hanford Inc.

Technical Editing and Document Services:

M. P. Curry Lockheed Martin Services, Inc.

Report Input and Contractor Review:

T. L. Baker Rust Federal Services Hanford Inc

T. G. Beam B\&W Hanford Co.

W. R. Brown Fluor Daniel Hanford, Inc.

F. M. Coony Rust Federal Services Hanford Inc.

A. E. Hill B\&W Hanford Co.

W. S. Josephson Rust Federal Services Hanford Inc.

O. S. Kramer Fluor Daniel Hanford, Inc.

S. S. Lowe Rust Federal Services Hanford Inc.

M. W. McCoy Pacific Northwest National Laboratory

A. G. Miskho Fluor Daniel Hanford, Inc.

D. E. Nestor Rust Federal Services Hanford Inc.

D. H. Nichols Fluor Daniel Hanford, Inc.

P. A. Powell Lockheed Martin Hanford Co.

G. S. Robinson Bechtel Hanford Inc.

J. N. Strode Lockheed Martin Hanford Co.

C.N. Villalobos B\&W Hanford Co.

P. J. Weaver B\&W Hanford Co.

U.S. Department of Energy, Richland Operations Office management review, coordination, and approval:

K. D. Bazzell

R. F. Guercia 
DOE/RL-97-14

This page intentionally left blank. 


\section{ACRONYMS AND ABBREVIATIONS}

\begin{tabular}{ll} 
ALARA & as low as reasonably achievable \\
BDAT & best demonstrated available technology \\
BEMR & baseline environmental management report \\
CAW & current acid waste \\
CCW & constituent concentrations in waste \\
CCWE & constituent concentration in the waste extract \\
CERCLA & Comprehensive Environmental Response Compensation \\
& and Liability Act of I980 \\
CFR & Code of Federal Regulations \\
CUU & CU column aqueous waste stream \\
CWC & Central Waste Complex \\
CXP & CX column aqueous waste stream \\
D2EHP & di(2-ethylhexyl) phosphoric acid \\
D\&AL & Development and Analytical Laboratories \\
D\&D & Deactivation and decommissioning \\
DBP & di-butyl phosphate \\
DOE & U.S. Department of Energy \\
DOE-HQ & U.S. Department of Energy-Headquarters \\
DSS & double-shell slurry \\
DSSF & double-shell slurry feed \\
DST & double-shell tank \\
Ecology & Washington State Department of Ecology \\
EIS & Environmental Impact Statement \\
EPA & U.S. Environmental Protection Agency \\
ETF & Effluent Treatment Facility \\
FFCAct & Federal Facilities Compliance Act of 1992 \\
FFTF & Fast Flux Test Facility \\
FR & Federal Register \\
FY & fiscal year \\
GTF & Grout Treatment Facility \\
HEPA & high-efficiency particulate air (filter) \\
HLV & high-level vault \\
HLW & high-level waste \\
HOC & halogenated organic carbon \\
HSW & high-salt waste \\
HWVP & Hanford Waste Vitrification Plant \\
IEMC & Interim Examination and Maintenance Cell \\
LBNL & Lawrence Berkeley National Laboratory \\
INEL & Idaho National Engineering Laboratory \\
LAW & low-activity waste \\
LDR & land disposal restriction \\
& \\
\hline
\end{tabular}




\section{ACRONYMS AND ABBREVIATIONS (cont)}

LERF

LLBG

LLW

LSA

LSW

MBP

NA

National Report

NCAW

NCRW

NEPA

NPH

ORNL

PCB

PFP

I PHMC

PNNL

PRF

PUREX

RCRA

REC

RL

RMC

RMW

SALDS

SRS

SST

TBD

TBP

TCLP

TOC

TOX

Tri-Party Agreement

TRU

TRUEX

TRUPACT

TRUSAF

TSCA

TWRS

WAC

WERF
Liquid Effluent Retention Facility

low-level burial grounds

low-level waste

low specific activity

low-salt waste

mono-butyl phosphate

not applicable

National Report on Prohibited Wastes and Treatment Options

(DOE 1990)

neutralized current acid waste

neutralized cladding removal waste

National Environmental Policy Act of 1969

normal paraffin hydrocarbon

Oak Ridge National Laboratory

polychlorinated biphenyl

Plutonium Finishing Plant

Project Hanford Management Contract

Pacific Northwest National Laboratory

Plutonium Reclamation Facility

Plutonium-Uranium Extraction (Plant)

Resource Conservation and Recovery Act of 1976

Radiochemical Engineering Cells

U.S. Department of Energy, Richland Operations Office

Remote Mechanical "C" Line

radioactive mixed waste

state-approved land-disposal structure

Savannah River Site

single-shell tank

to be determined

tri-butyl phosphate

toxic characteristic leach procedure

total organic carbon

total organic halide

Hanford Federal Facility Agreement and Consent Order

transuranic

transuranic extraction

transuranic package transporter

Transuranic Waste Storage and Assay Facility

Toxic Substances Control Act of 1976

Tank Waste Remediation System

Washington Administrative Code

Waste Experimental Reduction Facility 
DOE/RL-97-14

\section{ACRONYMS AND ABBREVIATIONS (cont)}

WESF

WIPP

I WRAP
Waste Encapsulation and Storage Facility

Waste Isolation Pilot Plant

Waste Receiving and Processing (Facility) (2336-W) 


\section{CONTENTS}

1.0 INTRODUCTION . . . . . . . . . . . . . . . . . . . . . . .

1.1 BACKGROUND AND PURPOSE $\ldots \ldots \ldots \ldots \ldots \ldots \ldots \ldots \ldots \ldots \ldots, 1-2$

1.2 ASSUMPTIONS $\ldots \ldots \ldots \ldots \ldots \ldots \ldots \ldots \ldots \ldots \ldots \ldots \ldots \ldots \ldots \ldots \ldots, 1-4$

1.3 SCHEDULE AND MECHANICS OF PLAN UPDATE $\ldots \ldots \ldots \ldots \ldots \ldots 1-6$

1.4 MILESTONE PLANNING PROCESS $\ldots \ldots \ldots \ldots \ldots \ldots \ldots \ldots \ldots \ldots, 1-7$

1.5 ACTIVITIES AND ACHIEVEMENTS $\ldots \ldots \ldots \ldots \ldots \ldots \ldots \ldots \ldots$

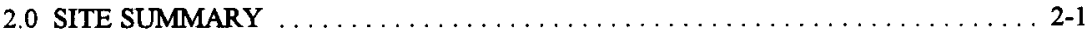

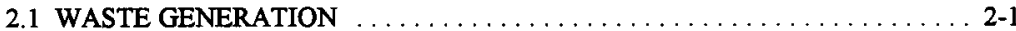

2.2 WASTE CHARACTERIZATION $\ldots \ldots \ldots \ldots \ldots \ldots \ldots \ldots \ldots \ldots \ldots \ldots \ldots, 2-2$

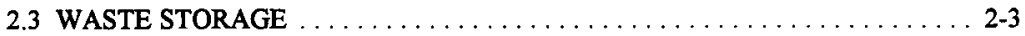

2.4 WASTE TREATMENT $\ldots \ldots \ldots \ldots \ldots \ldots \ldots \ldots \ldots \ldots \ldots \ldots \ldots \ldots \ldots \ldots \ldots \ldots, 4$

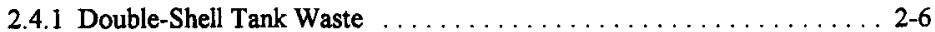

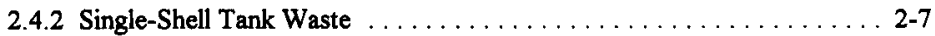

2.4.3 242-A Evaporator Process Condensate ............... 2-7

2.4.4 PUREX Storage Tunnels 1 and 2 and PUREX Containment Building Waste . . . . . . . . . .

2.4.5 Central Waste Complex Stored Low-Level, Transuranic, and Polychlorinated Biphenyl Waste; 224-T TRUSAF Stored Waste; and Suspect-Transuranic, Transuranic, and Other Regulated LLBG Waste $\ldots \ldots \ldots \ldots \ldots \ldots \ldots \ldots \ldots$

2.4.6 324 REC Waste . . . . . . . . . . . . . . . . . . . . 2-8

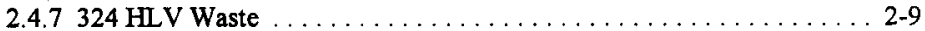

2.4.8 B Plant Cell 4 Waste . . . . . . . . . . . . . . . . . . . 2-9

2.4.9 B Plant Containment Building Storage $\ldots \ldots \ldots \ldots \ldots \ldots \ldots \ldots$ 2-9

2.4.10 B Plant Organic Waste $\ldots \ldots \ldots \ldots \ldots \ldots \ldots \ldots \ldots \ldots . . \ldots \ldots$

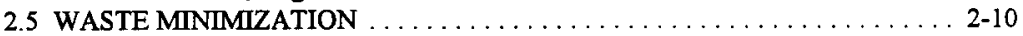

2.5.1 Waste Minimization Program Elements . . . . . . . . . 2-10

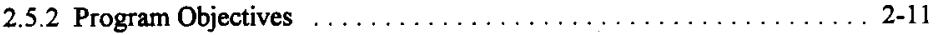

2.5.3 Facility-Specific Waste Minimization ............... 2-12

2.6 VARIANCES, SURFACE IMPOUNDMENT EXEMPTIONS, TREATMENT EQUIVALENCY PETITIONS, AND TIME EXTENSIONS $\ldots \ldots \ldots \ldots \ldots .2-13$ 
DOE/RL-97-14

CONTENTS (cont)

3.0 INDIVIDUAL WASTE STREAM INFORMATION $\ldots \ldots \ldots \ldots \ldots \ldots \ldots \ldots, 3-1$

3.1 DOUBLE-SHELL TANK WASTE $\ldots \ldots \ldots \ldots \ldots \ldots \ldots \ldots \ldots \ldots \ldots \ldots \ldots, 1$

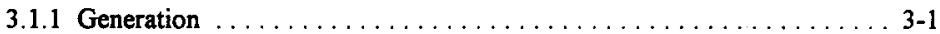

3.1 .2 Characterization $\ldots \ldots \ldots \ldots \ldots \ldots \ldots \ldots \ldots \ldots \ldots \ldots, 3-5$

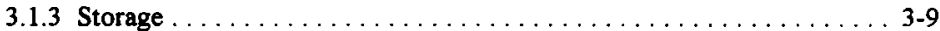

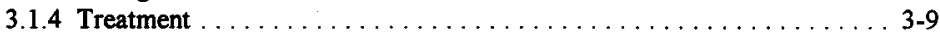

3.1.5 Waste Reduction ......................... 3-11

3.1.6 Variances, Equivalency Treatment Petitions, Time Extensions . . . . . 3-11

3.2 SINGLE-SHELL TANK WASTE $\ldots \ldots \ldots \ldots \ldots \ldots \ldots \ldots \ldots \ldots \ldots, 3-12$

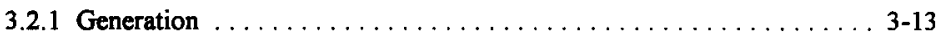

3.2.2 Characterization . . . . . . . . . . . . . . . . . . . . . . 3.14

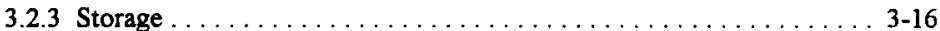

3.2 .4 Treatment .......... . . . . . . . . . . . . . . . . . 3-17

3.2.5 Waste Reduction . . . . . . . . . . . . . . . . . . . 3-18

3.2.6 Variances, Treatment Equivalency Petitions, Time Extensions . . . . . 3-18

3.3 242-A EVAPORATOR PROCESS CONDENSATE $\ldots \ldots \ldots \ldots \ldots \ldots \ldots$ 3-19

3.3.1 Generation . . . . . . . . . . . . . . . . . . . . . . . . .

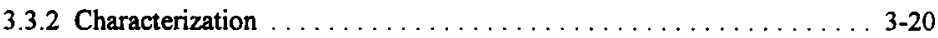

3.3.3 Storage $\ldots \ldots \ldots \ldots \ldots \ldots \ldots \ldots \ldots \ldots \ldots \ldots \ldots \ldots \ldots, 3-20$

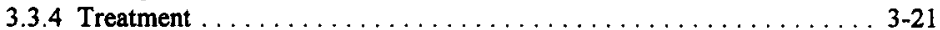

3.3.5 Waste Reduction . . . . . . . . . . . . . . . . . . . . . . . 3-22

3.3.6 Variances, Treatment Equivalency Petitions, Time Extensions $\ldots \ldots$ 3-22

3.4 PUREX STORAGE TUNNELS WASTE $\ldots \ldots \ldots \ldots \ldots \ldots \ldots \ldots \ldots, \mathbf{3 - 2 2}$

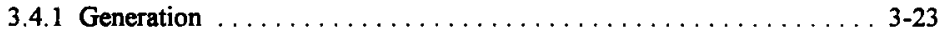

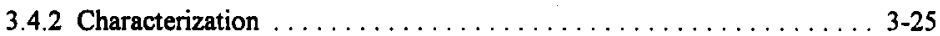

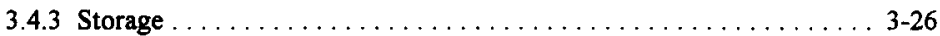

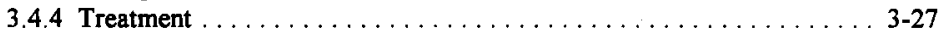

3.4.5 Waste Reduction ........................ 3-28

3.4.6 Variances, Treatment Equivalency Petitions, Time Extensions . . . . . 3-28

3.5 PUREX CONTAINMENT BUILDING (LEAD, CHROMIUM,

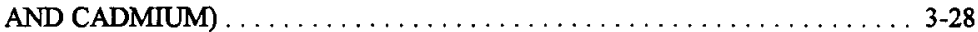

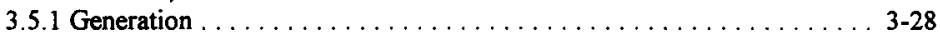

3.5.2 Characterization . . . . . . . . . . . . . . . . . . . . . 3-29

3.5.3 Storage $\ldots \ldots \ldots \ldots \ldots \ldots \ldots \ldots \ldots \ldots \ldots \ldots \ldots \ldots \ldots, 3-29$

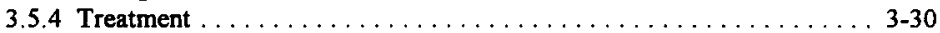

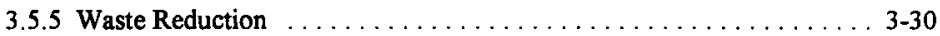

3.5.6 Variances, Treatment Equivalency Petitions, Time Extensions ..... 3-30 


\section{CONTENTS (cont)}

\subsection{CENTRAL WASTE COMPLEX STORED LOW-LEVEL, TRANSURANIC,} AND POLYCHLORINATED BIPHENYL WASTE $\ldots \ldots \ldots \ldots \ldots \ldots, 3-30$

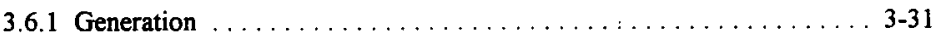

3.6.2 Characterization $\ldots \ldots \ldots \ldots \ldots \ldots \ldots \ldots \ldots \ldots \ldots \ldots \ldots \ldots \ldots, 3-33$

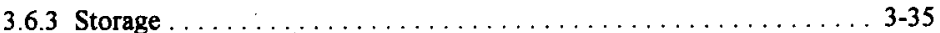

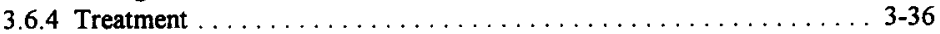

3.6.5 Waste Reduction $\ldots \ldots \ldots \ldots \ldots \ldots \ldots \ldots \ldots \ldots \ldots \ldots \ldots \ldots .3 .39$

3.6.6 Variances, Treatment Equivalency Petitions, Time Extensions . . . . . . 3-39

3.7 SUSPECT-TRANSURANIC AND TRANSURANIC WASTE AND OTHER REGULATED LLBG WASTE $\ldots \ldots \ldots \ldots \ldots \ldots \ldots \ldots \ldots, \mathbf{3 - 4 0}$

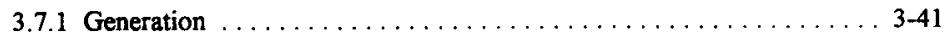

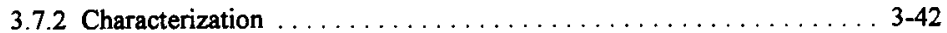

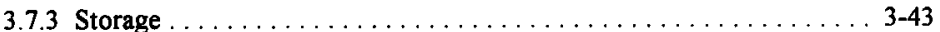

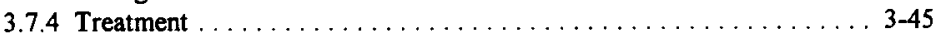

3.7.5 Waste Reduction .......................... 3-45

3.7.6 Variances, Treatment Equivalency Petitions, Time Extensions . . . . . 3-45

3.8 TRANSURANIC WASTE STORAGE AND ASSAY FACILITY

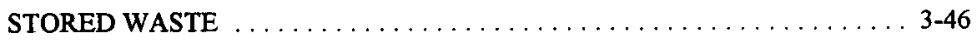

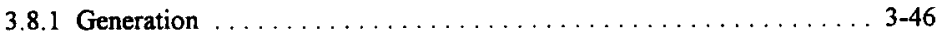

3.8.2 Characterization . . . . . . . . . . . . . . . . . . . . . 34

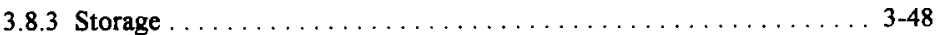

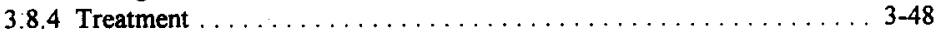

3.8.5 Waste Reduction . . . . . . . . . . . . . . . . . . . . 3-49

3.8.6 Variances, Treatment Equivalency Petitions, Time Extensions . . . . . 3-49

3.9324 RADIOCHEMICAL ENGINEERING CELLS WASTE $\ldots \ldots \ldots \ldots \ldots .3-49$

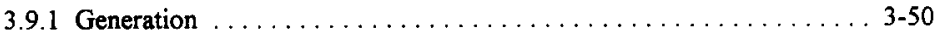

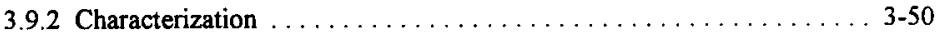

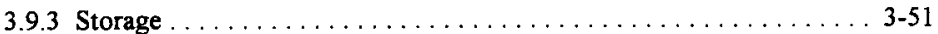

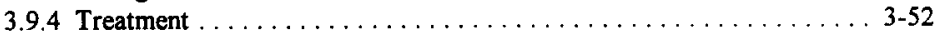

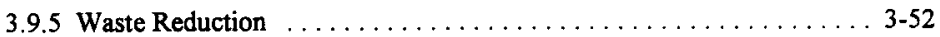

3.10324 HIGH-LEVEL VAULT TANK WASTE $\ldots \ldots \ldots \ldots \ldots \ldots \ldots \ldots, 3-52$

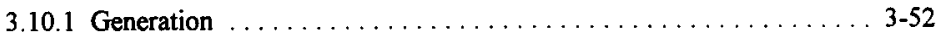

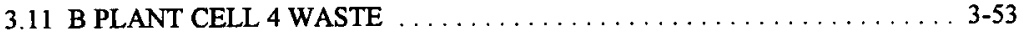

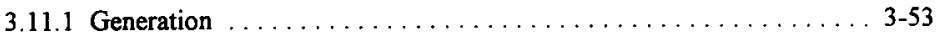

3.11.2 Characterization $\ldots \ldots \ldots \ldots \ldots \ldots \ldots \ldots \ldots \ldots \ldots \ldots \ldots \ldots \ldots \ldots \ldots \ldots, 3-53$

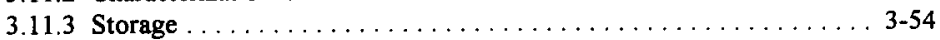

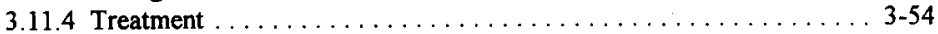

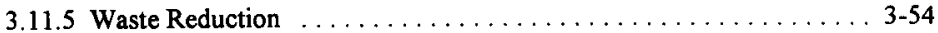

3.11.6 Variances, Treatment Equivalency Petitions, Time Extensions . . . . 3-54 
DOE/RL-97-14

CONTENTS (cont)

3.12 B PLANT CONTAINMENT BUILDING STORAGE $\ldots \ldots \ldots \ldots \ldots \ldots .3-55$

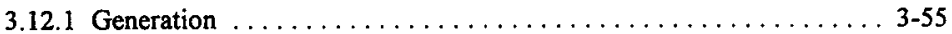

3.12.2 Characterization $\ldots \ldots \ldots \ldots \ldots \ldots \ldots \ldots \ldots \ldots \ldots \ldots \ldots, 3-55$

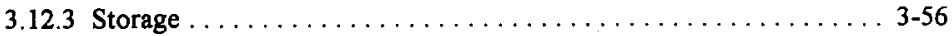

3.12 .4 Treatment ................................. 3-56

3.12.5 Waste Reduction ......................... 3-57

3.12.6 Variances, Treatment Equivalency Petitions, Time Extensions . . . . 3 3-57

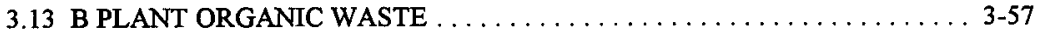

3.13.1 Generation .............................. 3-57

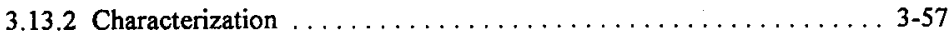

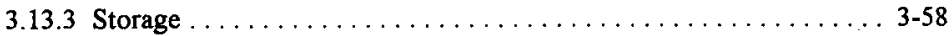

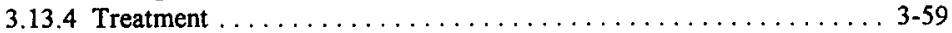

3.13.5 Waste Reduction ........................ 3-59

3.13.6 Variances, Treatment Equivalency Petitions, Time Extensions . . . . 3-59

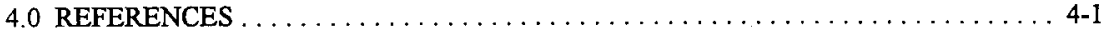

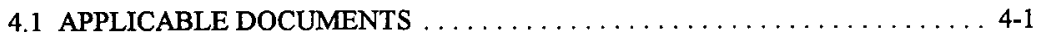

4.2 CODE OF FEDERAL REGULATIONS AND FEDERAL REGISTER $\ldots \ldots \ldots$ 4-4

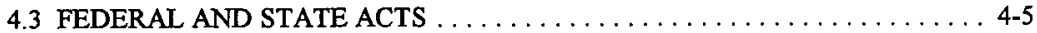

4.4 WASHINGTON STATE REGULATIONS $\ldots \ldots \ldots \ldots \ldots \ldots \ldots \ldots \ldots$ 4-5

APPENDIX

A. DATA IN NATIONAL DATABASE $\ldots \ldots \ldots \ldots \ldots \ldots \ldots \ldots \ldots \ldots \ldots \ldots \ldots \ldots$ 


\section{LIST OF FIGURES}

2-1 Operating Schedules for Units Managing Land Disposal Restricted Waste . . . . . F2-1.1

2-2 Hanford Tank Waste Remediation System . . . . . . . . . . . . . . . F2-2.1

I 2-3 Central Waste Complex Stored Waste and Retrievably Stored Waste

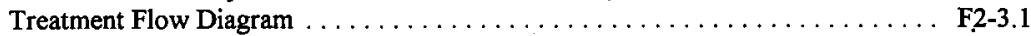

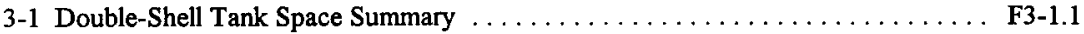

3-2 Waste Pretreatment Simplified Process Flow Diagram. . . . . . . . . . . . . F 3-2.1

3-3 Relative Proportions of Supernatant, Sludge, and

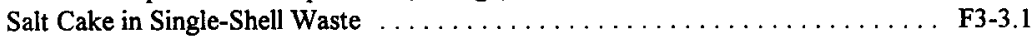

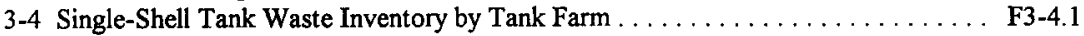

3-5 Process Flow Diagram for 242-A Evaporator . . . . . . . . . . . . . . . . F3-5.1

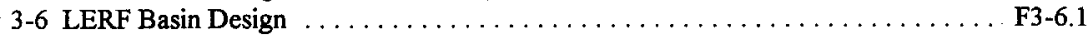

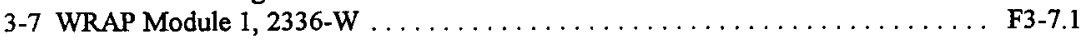

3-8 Waste Receiving and Processing Module 1 and Commercial Treatment . . . . . F 3-8.1

3-9 Typical Configuration of Suspect-TRU and TRU

Contact-Handled Waste to be Excavated. . . . . . . . . . . . . . . . F

3-10 Typical Configuration of a Retrievable Storage

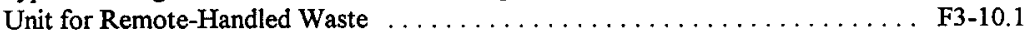

3-11 224-T Transuranic Storage and Assay Facility Floor Plan .............. F3-11.1

3-12 Historical Process Flow Diagram for B Plant

Strontium Solvent Extraction Process

F3-12.1 


\section{LIST OF TABLES}

1-1 Stream Names for the Hanford Land Disposal Restrictions Plan for Mixed Waste .

1-2 Streams no Longer Included in Report

2-1 Summary of Annual Waste Generation Projections $\ldots \ldots \ldots \ldots \ldots \ldots \ldots$ T2-1.1

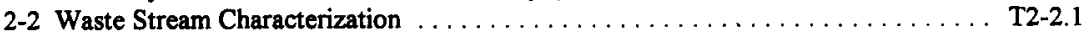

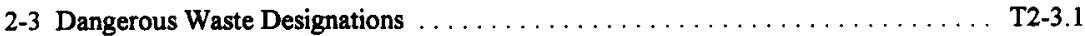

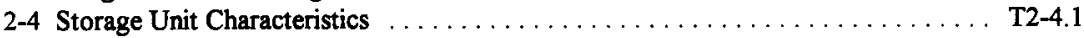

2-5 Stored Waste Characteristics . . . . . . . . . . . . . . . . . . T2-5.1

2-6 Treatment of Land-Disposal-Restricted Waste for Disposal . . . . . . . . . . T2-6.1

2-7 Waste Reduction Activities for Hanford Site

Land Disposal Mixed Waste

3-1 Estimated Mass of Nonradioactive Chemical Components of Single-Shell and Double-Shell Tank Wastes.

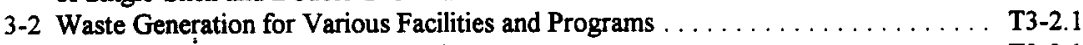

I 3-3 Hanford Site Single-Shell Tank Releases ..................... T3-3.1

3-4 Plutonium-Uranium Extraction Plant Storage Inventories . . . . . . . . . . . T3-4.1

1 3-5 Forecasted Low-Level and Transuranic Waste to be Stored at the Central Waste

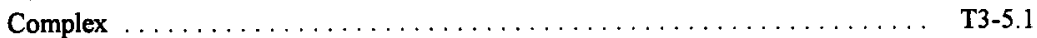

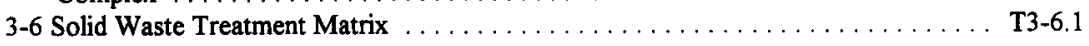


DOE/RL-97-14

This page intentionally left blank. 


\subsection{INTRODUCTION}

The baseline land disposal restrictions (LDR) plan was prepared in 1990 in accordance with the Hanford Federal Facility Agreement and Consent Order (commonly referred to as the

1 Tri-Party Agreement) Milestone M-26-00 (Ecology et al. 1989). The text of this milestone is below.

"LDR requirements include limitations on storage of specified hazardous wastes (including mixed wastes). In accordance with approved plans and schedules, the U.S. Department of Energy (DOE) shall develop and implement technologies necessary to achieve full compliance with LDR requirements for mixed wastes at the Hanford Site. LDR plans and schedules shall be developed with consideration of other action plan milestones and will not become effective until approved by the U.S. Environmental Protection Agency (EPA) (or Washington State Department of Ecology [Ecology]) upon authorization to administer LDRs pursuant to Section 3006 of the Resource Conservation and Recovery Act of 1976 (RCRA). Disposal of LDR wastes at any time is prohibited except in accordance with applicable LDR requirements for nonradioactive wastes at all times. The plan will include, but not be limited to, the following:

- Waste characterization plan

- Storage report

- Treatment report

- Treatment plan

- Waste minimization plan

- A schedule depicting the events necessary to achieve full compliance with LDR requirements

- A process for establishing interim milestones."

I The original plan was published in October 1990. This is the seventh of a series of annual updates required by Tri-Party Agreement Milestone M-26-01. A Tri-Party Agreement change request approved in March 1992 changed the annual due date from October to April and consolidated this report with a similar one prepared under Milestone M-25-00. The reporting period for this report is from April 1, 1996, to March 31, 1997.

The 1990 baseline plan was a follow-on document to both the National Report on Prohibited Wastes and Treatment Options (DOE 1990) (commonly referred to as the National Report), which identified all solvent (40 Code of Federal Regulations [CFR] 268.30) and 
California List (40 CFR 268.32) wastes that are restricted from land disposal, and a subsequent effort by DOE (WHC 1990d) to identify any additional waste that was restricted from land disposal as a result of First-, Second-, and Third-Third LDRs promulgation ( 55 Federal Register [FR] 22520).

This year's report will again be used as the Hanford Site's equivalent to the final Site Treatment Plan (STP) submitted to the U.S. Department of Energy-Headquarters (DOE-HQ) as required by the Federal Facility Compliance Act of 1992 (FFCAct). Although the State of Washington and the EPA concurred that the U.S. Department of Energy, Richland Operations Office (RL) was not obligated to complete a site treatment plan, RL and the Hanford Site contractors have been participating in the FFCAct process by providing data and cost information to support a complex-wide effort to prioritize treatment projects. This report includes an appendix that contains site treatment plan inventory data prepared to support the FFCAct.

\subsection{BACKGROUND AND PURPOSE}

On September 19, 1989, DOE entered into a federal facilities compliance agreement with the EPA and the Colorado Department of Health regarding the storage of certain radioactive mixed wastes at the Rocky Flats Plant. The agreement required the DOE to prepare and submit the National Report to the EPA. This report (DOE 1990) was submitted to EPA in January 1990. It included information on all DOE sites that store radioactive mixed waste subject to the LDRs in effect at the time of report preparation.

The EPA has promulgated various new LDR rules since the Rocky Flats compliance agreement. (Recent major LDR rulemakings, the phase III rules, in the 61 FR and 62 FR series, are listed in Chapter 4.0, "References".) The LDRs apply to the hazardous component of mixed wastes. Of particular interest at federal facilities is the storage prohibition of RCRA Section 3004(j).

Washington State promulgated new regulations that affect designation of waste generated and waste in storage after November 20, 1995, [WAC 173-303-070(3)(a) and 173-303-070(5)]. Federal waste codes (e.g., D001) are assigned to a waste first, followed by state codes (e.g., WT01). Based on these amendments, the state code is not assigned in many cases. (The new regulations have not been applied to waste in storage that was generated before the effective date The new regulations will be applied to stored waste that is moved to a new storage location after the effective date.)

By passing the FFCAct, Congress incorporated provisions for the storage of mixed wastes at DOE facilities. Among these provisions was a 3-year delay in the effective date of the waiver of immunity for violations of the land disposal storage prohibition [RCRA Section 3004(j)] with respect to mixed waste storage at DOE facilities. The DOE can continue to avoid penalties after the expiration of the 3-year extension if certain plans are developed and submitted pursuant to RCRA Section 3021(b). Plans are not required for DOE sites that are subject to an existing State permit, agreement, or order that establishes a schedule for treatment. Because the Tri-Party 
Agreement addresses compliance with RCRA Section 3021(b)(5), the requirements of RCRA I Section 3021(b) are not applicable to mixed wastes in storage on the Hanford Site. In addition, I 61 FR 18591 further extended the RCRA 3004(j) storage prohibition effective date. For DOE I sites in compliance with the FFCAct, the extension is indefinite.

This report describes the generation and management of LDR mixed waste generated, treated, and stored at the Hanford Site. Discussions focus on the hazardous aspects of mixed wastes, although treatment, storage, and disposal are frequently complicated by the radioactive components. This report discusses the LDR mixed waste managed at the Hanford Site by a combination of point of generation and current storage locations. The waste is separated into groups based on its future treatment before disposal. This grouping results in the definition of

| 13 groups or streams of LDR waste. The 13 stream names used for this plan are shown in Table 1-1. Where a "stream" is actually in a storage unit, the waste streams that make up the storage unit are discussed in this report as applicable.

The 13 waste streams identified for this report combine several of the waste streams identified in the National Report and the case-by-case extension petition. The National Report included solvent waste (40 CFR 268.30) and California List (40 CFR 268.32) wastes, whereas the case-by-case petition was to include all nonsolvent waste that was restricted from land disposal. This report encompasses the Hanford Site-specific aspects of the National Report (DOE 1990) and the case-by-case petition, as well as newly identified LDR waste.

Discussions with the regulators were completed in 1996 regarding major modifications to the Tri-Party Agreement milestones that address solid wastes. The new milestones were created in partial fulfillment of treatment requirements found in Milestone M-26-00, which constitutes an existing Agreement or Order for treatment of mixed waste under the Federal facility Compliance Act of 1992, and as companion documentation to this report. These new milestones govern the acquisition of new facilities, modification of planned facilities necessary for the storage, and/or disposal of treated and immobilized tank wastes (Milestone M-90); storage, processing, and disposal of Hanford Site transuranic and transuranic mixed waste and low-level mixed waste (Milestone M-91); and, storage, treatment/processing, and disposal of Hanford Site cesium and strontium capsules, unirradiated uranium, bulk sodium, and 300 Area special case waste (Milestone M-92).

None of the materials covered by Milestone M-92 were considered waste at the time this report was prepared. Generally, they are still classified as "product," with future use not finalized. This is expected to change to classification as waste for at least some of the materials. Those materials will be added to future revisions of this report as appropriate. The materials that will be classified as waste will be determined as part of the process of completing the M-92 series of milestones.

Milestone M-91 covers remote-handled low-level mixed waste (RH-LLMW) treatment in a Project Management Plan that is to be completed in June 1999. M-91 also targets the award of commercial contract for RH and large-size LLMW in October 2003 and requires onsite facilities and initiation of treatment by June 2008 . 
Milestone M-91 requires that a TRU/TRUM Project Management Plan be completed by June 2000, and specifies a target date of September 2003 for award of commercial contracts to process RH and large-size TRU/TRUM.

Some waste streams that were included in previous LDR reports are no longer included as separate waste streams in this report. The names of these streams, sources of the waste, and reasons for deletion from the report are summarized in Table 1-2.

\subsection{ASSUMPTIONS}

This section lists key milestones and assumptions used to prepare this plan.

RL has negotiated changes to the Tri-Party Agreement to allow for the privatization of the low-activity waste (LAW) pretreatment and immobilization. This approach will allow private companies to treat the tank waste and return the treated waste products to DOE. To integrate the privatization process, the LAW pretreatment process will be included with the LAW immobilization process.

Change request M-50-95-01 approved 7/24/96 deleted all uncompleted interim milestones of M-50-00 that pertained to the design, construction, and operation of a pretreatment facility for tank waste. This work was moved to Tri-Party Agreement Milestone M-60-06.

Change request M-60-95-30 approved 7/24/96 deleted all uncompleted interim milestones of M-60-00 that pertained to the design, construction, and operation of a LAW vitrification facility. The change request established a new primary path that includes new interim and major milestone due dates for a two phase approach to the pretreatment and immobilization of the lowactivity tank waste. This primary path allows for the solicitation and award of a private contract to design, construct, and operate a LAW pretreatment and immobilization facility. The change request also establishes a new Tri-Party Agreement milestone, M-61-00, which describes interim and major milestones for an alternative path, which would be enforceable if DOE abandons the primary path (M-60-00) for any reason. This milestone will be deleted when the primary path milestones are completed (WHC 1994a).

are as follows, including approved change requests.

Other significant Tri-Party Agreement milestones related to the management of LDR waste

- Complete separation of tank waste into low-activity and high-activity fractions by December 2028 (M-50-00). This milestone includes beginning operations by December 2004 (M-50-02) to support operation of LAW treatment facility. These milestone activities were deleted per change request M-50-95-01 approved on $7 / 24 / 96$.

- Complete vitrification of LAW by December 2028 (M-60-00). The waste treatment facility (vitrification) will begin operations in June 2005 (M-60-05). These milestone 
activities were deleted per change request M-60-95-03 approved on 7/24/96. New milestone dates were established: complete pretreatment and immobilization of Hanford LAW by December 2024 (M-60-00). Start hot operations of two LAW pretreatment and immobilization facilities by December 2002 (M-60-12).

- Complete vitrification of high-level waste (HLW) by December 2028 (M-51-00). Operation of the HLW Vitrification Plant will begin in December 2009 (M-51-03).

- Complete single-shell tank (SST) interim stabilization by September 2000 (M-41-00).

- Complete closure of all SST farms by September 2024 (M-45-00). This milestone includes a requirement to initiate tank waste retrieval from one SST by December 2003 (M-45-05T1).

- Issue Tank Characterization Reports for all 177 SSTs and double-shell tanks (DST) by September 1999 (M-44-00).

- Complete construction and initiate operations of expanded laboratory hot cells for high-level mixed waste by June 1994 (M-11-00). This milestone is complete.

- Complete Waste Receiving and Processing (WRAP) Facility, Module 1, construction and initiate operations by March 1997 (M-18-00). This milestone is complete.

- Initiate treatment of contact-handled low-level mixed waste by September 1999 (M-19-01), and complete treatment and/or direct disposal of at least 1,644 cubic meters of contact-handled low-level mixed waste by September 2002 (M-19-00). The M-19 change request that establishes these alternatives to the construction and operation of the operation of the WRAP 2A facility received final approval in October 1996.

- Complete closure of the non-permitted mixed waste units in the 324 Building Radiochemical Engineering Cells (REC) B Cell, REC D Cell, and the HLV (M-89-00). A date for completion of this milestone will be established immediately following Ecology approval of the REC/HLV closure plan (M-20-55).

- Complete removal of 324 Building Radiochemical Engineering Cells (B Cell) mixed waste and equipment by May 31, 1999, (M-89-02).

- Complete B Plant facility transition phase and initiate the surveillance and maintenance phase by September 1999 (M-82-00). This milestone includes removing organic waste from the B Plant canyon by June 1997 (M-82-03), documenting any hazardous substances or dangerous waste remaining in B Plant by June 1998 (M-82-07), and completing disposition of the organic waste by September 1998 (M-82-08). 
The following are key assumptions that have been used to develop the treatment plans and schedules for DST waste (WHC 1990a) and assumptions related to the use of tank space.

- The pretreatment methods to be developed will include acceptable technology to separate the waste into low- and high-activity streams so that the bulk of chemical waste is in the low-activity stream and the bulk of radionuclides are in the highactivity stream.

- Pretreated waste from all DSTs and SSTs will be provided to the LAW and HLW vitrification facilities, using selective blending if necessary.

- The level of cyanides and organics in DST and SST waste received from pretreatment will be treatable by vitrification, and the glass waste forms will fully comply with leachability requirements or appropriate variances will be obtained.

- Space in DSTs will be available to support DST and SST waste disposal activities.

- During Plutonium-Uranium Extraction (PUREX) Plant deactivation, no new PUREX aging waste, PUREX process condensate, or PUREX ammonia scrubber waste will be generated. In calendar year (CY) 1996, no waste was transferred to the aging waste tanks, and none will be sent in future years.

- Liquid waste from SSTs will continue to be transferred to DSTs as part of the stabilization program for the SSTs.

- Process condensate from the 242-A Evaporator and other hazardous waste water, including liquid effluents from tank waste pretreatment and vitrification will be treated in the 200 Area ETF.

\subsection{SCHEDULE AND MECHANICS OF PLAN UPDATE}

Information in the baseline plan will be updated by additional future annual reports in accordance with Tri-Party Agreement Milestone M-26-01. The annual reports include the following:

- Addition of new LDR waste streams as they are identified or regrouped

- Revision of the stream generation rates to reflect current operating plans and schedules

- Revision to treatment plans and schedules to reflect further defined waste treatments and treatment schedules 
- Revision to the stream characterizations to reflect additional sample analyses or process changes

- Revision to the compliance status of the units to reflect future compliance assessments and permitting activities

- Reevaluation of the adequacy of the capacity of current units for the storage of LDR waste

- Addition of new or proposed milestones, as applicable

- Changes in the configuration of the mixed waste complex required under the FFCAct

- Future LDR report updates will include new mixed waste streams as they are identified or declared to be waste. These may include cesium/strontium capsules, bulk sodium waste, and 300 Area special-case waste. Tri-Party Agreement milestones for these waste types have been established in the M-92 milestone series.

\subsection{MILESTONE PLANNING PROCESS}

Milestones and work schedules for activities related to the management of LDR mixed waste will be consistent with the work schedules contained in Appendix D of the Tri-Party Agreement (Ecology et al. 1989) and the annual update to the work schedule. The scope of these schedules includes interim milestones and additional target dates to accomplish the major milestones contained in Section 2.0 of the Tri-Party Agreement. Summary milestone schedules for activities related to the management of LDR mixed waste are discussed in Chapter 3.0 of the Tri-Party Agreement. Any new or additional LDR milestones, as well as changes to approved LDR milestone schedules, will be implemented via the Change Control System process defined in Section 12.0 of the Tri-Party Agreement.

Tri-Party Agreement Milestone M-26-01 also requires that appropriate new milestones be proposed through this annual report. No new milestones are proposed in this report for this reporting period.

The LDR milestone planning process exercised by DOE and its contractors also involves consideration of DOE and federal budget process, integration with other concurrent Hanford Site operations (including waste management and environmental restoration activities), and overall sitewide regulatory compliance and coordination with other milestone initiatives described in the Tri-Party Agreement. Because these planning elements are numerous and complex, coordination and resolution of issues will be accomplished through the ongoing project managers' and unit managers' meetings within the broader framework provided by Section 8.0 of the Tri-Party Agreement. Also, LDR waste management activities will be included, as appropriate, in Tri-Party Agreement milestone review meetings. 


\subsection{ACTIVITIES AND ACHIEVEMENTS}

This section summarizes major activities and accomplishments related to compliance with | LDRs from about April 1, 1996, through March 31, 1997.

- The 242-A Evaporator completed one campaign in 1996. The volume of tank waste stored in the DSTs was reduced by 4,200 cubic meters, and 4,800 cubic meters of process condensate were produced.

- The 200 Area ETF treated 37,500 cubic meters of process condensate stored in the LERF.

- Nine additional submarine reactor compartments were received for burial in the 200 East Area, bringing the total to 62 .

- WRAP Module 1, Project W-026, began operations on March 12, 1997 in advance of Tri-Party Agreement Milestone M-18-00 requirements. Non-destructive assay and non-destructive examination will be the first operational facilities. Construction of WRAP Module 1 was completed in June 1996 in accordance with the requirements of Tri-Party Agreement Milestone M-18-01.

- In June 1996 it was discovered by Lawrence Berkeley Laboratory that 77 drums of low-level waste shipped to the Hanford Site from the National Tritium Labeling Facility in 1989, 1990, and 1995 may contain organic solvents. On June 28, 1996, Ecology issued a Voluntary Compliance Order to RL and WHC about this issue. A timely response was submitted to Ecology on September 27, 1996, completing all required contractor actions about this issue.

- Macroencapsulation of three air lances from tank 241-SY-101 and three thermocouples from 241-AZ-101 was completed at T Plant on September 30, 1996. This represents 30 cubic meters of remote-handled mixed waste that is treated and ready for disposal.

- Final approval of the M-19 Tri-Party Agreement Change Request, which establishes alternatives to building and operating the WRAP $2 \mathrm{~A}$ facility, was received in October 1996. The revised M-19 calls for the use of a combination of treatment contracts, direct disposal, and smaller onsite treatment projects to accomplish the WRAP 2A mission.

- In 1995, DOE, Ecology, and EPA agreed to enter into negotiations on matters relating to storage, treatment, and/or disposal of Hanford Site solid waste and materials. In 1996, negotiations were concluded on three new sets of milestones. The M-90 series of milestones governs the acquisition of new and modified facilities for the storage and/or disposal of immobilized tank waste. The M-91 series governs the acquisition of new and modified facilities for the storage, processing, and disposal 
of TRU and transuranic mixed (TRUM), LLMW, and Greater than Class 3 (GTC3) LLW and LLMW. The M-92 series governs the acquisition of new and modified facilities for storage, treatment and processing, and disposal of cesium and strontium capsules, unirradiated uranium, bulk sodium, and 300 Area special-case waste. The new milestones and resulting plans are incorporated into this report as appropriate.

- Construction on Project W-112, "Enhanced Radioactive and Mixed Waste Storage Facility, Phase V" was completed in January 1997. The facility comprises three buildings with a total compliant storage capacity of about 2,800 cubic meters.

- In February 1997, Ecology granted a "contained-in" determination for 828 backlog waste soil drums from the tank farms. This determination allowed waste codes F001 through F005 to be removed from these drums. "State only" dangerous waste codes are also not required for PCBs in the waste, however all PCB soils are still required to be managed in accordance with the Toxic Substances Control Act of 1976 (TSCA). This determination was the result of review of analytical data from drum soil samples.

- Tri-Party Agreement Milestone M-89-01 was completed with the removal of the mixed-waste liquids from the HLV and subsequent processing in the REC D-Cell. The HLV tanks were rinsed and the rinse water was also removed and processed in D-Cell. Solid treatment residues from the processing of the liquid waste are being managed in the REC as mixed waste. It is proposed that their disposal be coordinated with the high-activity mixed waste being collected in the REC B-Cell to minimize disposal costs.

- Removal of tributyl phosphate/normal paraffin hydrocarbon at PUREX was completed in June 1996. This activity involved shipping approximately 11,000 liters of the material to the Diversified Scientific Services, Inc. facility in Tennessee for incineration.

- All waste, except for some containerized waste, has been removed from PUREX in support of PUREX stabilization. No additional waste, except for some containerized waste, will be generated at PUREX until waste is removed from the railroad tunnels or the facility is in the disposition phase.

- Significant progress was made on Tri-Party Agreement Milestone M-89-02 when all containerized mixed waste and special-case waste was removed from the 324 Building and shipped to PUREX Storage Tunnel 2 (a permitted storage facility).

- Forty tank characterization reports for high-level waste tanks were completed in accordance with Milestone M-44-09. Samples have been taken from 121 of the 177 DSTs and SSTs. 
- Work continues on the DST Dangerous Waste Permit Application (DOE/RL-90-39). During 1996, workshops were held with Ecology on Chapters 1, "Part A"; 5 , "Minimization"; 9, "Exposure Information Report"; 10, "Waste Minimization"; and 12 "Reporting and Recordkeeping." Notices of deficiency for these chapters were tentatively closed out pending incorporation of changes into the permit application. Also, a workshop was held on Chapter 11, "Closure and Postclosure Requirements." Comments from the workshop resulted in Chapter 11 being rewritten. Significant work has also been completed on Chapter 6, "Procedures to Prevent Hazards," and the appended DST System Inspection Schedule. Chapters 6 and 11 will be transmitted to Ecology in 1997 for review.

- Vapor characterization was completed for all ferrocyanide watch list tanks (M-40-03) and all organic watch list tanks (M-40-08).

- During 1996 three tank farms, TY, TX, and BX, were declared "Controlled, Clean, and Stable." The criteria for controlled, clean, and stable are as follows:

- All pumpable liquid has been removed for the tanks

- All potential tank opening have been sealed

- All abandoned equipment has been removed

- All reusable equipment is placed in compliant storage

- All surface contamination has been cleared up

- Automated surveillance operations have been installed

- Surveillance data will be recorded electronically.

- The tank waste treatment program is being privatized. Lockeed Martin Advanced Environmental Systems and British Nuclear Fuels Limited were selected in 1996 to begin proof-of-concept and commercial demonstration activities.

- New data confirm that radioactive cesium-137 is present beneath the SX tank farm, the location of 15 SSTs in the 200 West Area. The radioactive material was present in a new borehole drilled adjacent to tank SX-109 in December 1996. The borehole was drilled in response to recommendations by an independent panel of experts. Cesium was detected in the new borehole at 40 meters, 15 meters deeper than expected based on computer models used to predict contaminant movement through the vadose zone. In previously drilled boreholes, cesium was detected at 38 meters. Tank SX-109 was declared a leaking tank in 1965 . The estimated volume of leakage from SX-109 is 38,000 liters. Pumpable liquids were reduced to less than 95,000 liters (interim stabilization) in 1981.

- The 18 SSTs on the Ferrocyanide Watch List were removed during 1996 (4 in June and 14 in September 1996). The concern about these tanks had been that because of the ferrocyanide and sodium nitrate content, uncontrolled exothermic reactions could result. It has been concluded that the cyanides have been destroyed through chemical processes in the tanks. 
- Organic solvent decontamination washes of B Plant organic waste to achieve chemical separation of radionuclides were completed in June 1996 to meet Tri-Party Agreement target milestone M-32-07-T05. The B Plant organic waste was transferred from the B Plant facility to permitted storage tanks outside of the facility in February 1997 completing Tri-Party Agreement milestone M-82-03. 
DOE/RL-97-14

This page intentionally left blank. 
Table 1-1. Stream Names for the Hanford Land Disposal

Restrictions Plan for Mixed Waste. (2 sheets)

\begin{tabular}{|c|c|}
\hline Stream name & Waste source \\
\hline 1. DST Waste & $\begin{array}{l}\text { Widely varying wastes from chemical separations } \\
\text { processes (e.g., PUREX Plant, PFP, cesium and } \\
\text { strontium separations) and related support } \\
\text { facilities used from } 1970 \text { to date. }\end{array}$ \\
\hline 2. SST Waste (inventory) ${ }^{2}$ & $\begin{array}{l}\text { Waste from spent nuclear fuel processing and } \\
\text { related support facilities between } 1944 \text { and } 1980 .\end{array}$ \\
\hline 3. 242-A Evaporator Process Condensate & $\begin{array}{l}\text { Condensed vapor from concentrating } \\
\text { DST waste. }\end{array}$ \\
\hline $\begin{array}{l}\text { 4. PUREX Storage Tunnel Waste } \\
\text { a. mercury } \\
\text { b. lead } \\
\text { c. silver } \\
\text { d. cadmium } \\
\text { e. Chromium } \\
\text { f. Barium } \\
\text { g. Mineral oil }\end{array}$ & $\begin{array}{l}\text { Mercury sealed in discarded PUREX fuel } \\
\text { dissolvers (Tunnel 2) } \\
\text { Lead from discarded equipment and shielding } \\
\text { and } 324 \text { Building waste (Tunnels } 1 \text { and 2) } \\
\text { Silver from discarded silver reactors (Tunnel 2) } \\
\text { Cadmium associated with shielding and } \\
\text { moderators and } 324 \text { Building waste (Tunnel 2) } \\
\text { Chromium as corrosion byproduct and } 324 \\
\text { Building waste (Tunnels 1 and 2) } \\
324 \text { Building waste (Tunnel 2) } \\
324 \text { Building waste (Tunnel 2). }\end{array}$ \\
\hline 5. PUREX Containment Building & $\begin{array}{l}\text { Chromium-contaminated debris from E Cell floor } \\
\text { stored in F Cell. }\end{array}$ \\
\hline $\begin{array}{l}\text { 6. Central Waste Complex Stored Low- } \\
\text { Level, Transuranic, and PCB Waste }\end{array}$ & $\begin{array}{l}\text { Onsite and offsite solid wastes from many } \\
\text { generators, primarily from routine operations } \\
\text { after } 1987 \text {. }\end{array}$ \\
\hline $\begin{array}{l}\text { 7. Suspect-Transuranic and Transuranic } \\
\text { Waste and Other Regulated Low-Level } \\
\text { Burial Ground Waste (inventory) }\end{array}$ & $\begin{array}{l}\text { Containers of contaminated debris generated on } \\
\text { site and off site up to } 1987 \text {. }\end{array}$ \\
\hline 8. 224-T TRUSAF Stored Waste & $\begin{array}{l}\text { Transuranic waste from onsite and offsite, } \\
\text { packaged for eventual WIPP disposal. }\end{array}$ \\
\hline
\end{tabular}


Table 1-1. Stream Names for the Hanford Land Disposal Restrictions Plan for Mixed Wastes. (2 sheets)

\begin{tabular}{|l|l|}
\hline \multicolumn{1}{|c|}{ Stream name } & \multicolumn{1}{c|}{ Waste source } \\
\hline $9 . \quad 324$ REC & $\begin{array}{l}\text { Variety of high-activity radioactive wastes, } \\
\text { containing regulated quantities of predominantly } \\
\text { toxic heavy metals, generated during research } \\
\text { and development activities ongoing since the } \\
\text { mid-1960s and the processing of 324 Building } \\
\text { HLV waste. }\end{array}$ \\
\hline 10.324 HLV & $\begin{array}{l}\text { High-activity radioactive waste solutions that are } \\
\text { corrosive and contain regulated quantities of } \\
\text { toxic heavy metals generated during research and } \\
\text { development activities ongoing since the mid- } \\
\text { 1960s. This stream has been treated and no } \\
\text { longer exists as of October 1, 1996. }\end{array}$ \\
\hline 11. B Plant Cell 4 Waste & $\begin{array}{l}\text { Containers of mixed and/or highly radioactive } \\
\text { solid waste (no free liquids) generated in the } \\
\text { WESF hot cells since 1988. }\end{array}$ \\
\hline 12. B Plant Containment Building Storage & $\begin{array}{l}\text { Discarded and failed process jumpers and } \\
\text { equipment from B Plant operational processes } \\
\text { that are stored in the B Plant canyon deck and in } \\
\text { the process cells. }\end{array}$ \\
\hline & $\begin{array}{l}\text { Organic solvent waste from the strontium } \\
\text { solvent extraction process that operated in } \\
\text { B Plant from 1968 to 1979. }\end{array}$ \\
\hline 13. B Plant Organic Waste &
\end{tabular}

'Waste stream no longer being generated.

CWC = Central Waste Complex.

DST = Double-shell tank.

FFTF $=$ Fast Flux Test Facility.

HLV = High-Level Vault.

PCB = Polychlorinated biphenyl.

PFP = Plutonium Finishing Plant.

PUREX = Plutonium-Uranium Extraction (Plant).

REC = Radiochemical Engineering Cells.

SST = Single-shell tank.

TRUSAF = Transuranic Waste Storage and Assay Facility.

WESF $=$ Waste Encapsulation and Storage Facility.

WIPP $=$ Waste Isolation Pilot Plant. 
Table 1-2. Streams no Longer Included in Report.

\begin{tabular}{|c|c|c|}
\hline Stream name & Waste source & $\begin{array}{l}\text { Reason for deletion from } \\
\text { LDR Report }\end{array}$ \\
\hline PUREX Plant Aging Waste & $\begin{array}{l}\text { First extraction column } \\
\text { fission products from PUREX } \\
\text { plant. }\end{array}$ & $\begin{array}{l}\text { Waste no longer generated. } \\
\text { Inventory is in DSTs. }\end{array}$ \\
\hline $\begin{array}{l}4843 \text { Sodium Storage Facility } \\
\text { Waste }\end{array}$ & $\begin{array}{l}\text { Waste sodium from FFTF } \\
\text { operations. }\end{array}$ & $\begin{array}{l}\text { Waste no longer generated. } \\
\text { Inventory is now in storage at } \\
\text { the CWC and TSD unit is } \\
\text { undergoing closure. }\end{array}$ \\
\hline $\begin{array}{l}\text { PUREX Ammonia Scrubber } \\
\text { Waste }\end{array}$ & $\begin{array}{l}\text { Waste generated from } \\
\text { adsorption of gaseous } \\
\text { ammonia from fuel processing } \\
\text { operations at the PUREX } \\
\text { Plant. }\end{array}$ & $\begin{array}{l}\text { Waste no longer generated. } \\
\text { Inventory in DSTs. }\end{array}$ \\
\hline PUREX Process Condensate & $\begin{array}{l}\text { Condensed vapors from } \\
\text { PUREX Plant operations. }\end{array}$ & $\begin{array}{l}\text { Waste no longer generated. } \\
\text { Inventory in DSTs. }\end{array}$ \\
\hline Hexone Waste & $\begin{array}{l}\text { Hexone that had been planned } \\
\text { for use in the 202-S solvent } \\
\text { extraction process. }\end{array}$ & $\begin{array}{l}\text { Hexone has been incinerated } \\
\text { off site. }\end{array}$ \\
\hline $\begin{array}{l}\text { 183-H Solar Evaporation } \\
\text { Basins Waste }\end{array}$ & $\begin{array}{l}\text { Containerized solids retrieved } \\
\text { from } 183-\mathrm{H} \text { Area Solar } \\
\text { Evaporations Basins, } \\
\text { generated from } 300 \text { Area fuel } \\
\text { fabrication waste from } 1973 \\
\text { to } 1985 \text {. }\end{array}$ & $\begin{array}{l}\text { Closure Plan activities for the } \\
\text { TSD unit are complete. Unit } \\
\text { is in postclosure care. } \\
\text { Process waste inventory is } \\
\text { now in storage at the CWC. } \\
\text { Debris and soil removed } \\
\text { during remediation are not } \\
\text { designated as mixed waste. }\end{array}$ \\
\hline 303-K Stored Waste & $\begin{array}{l}\text { Temporary storage of } \\
300 \text { Area fuel fabrication } \\
\text { solid and liquid waste. }\end{array}$ & $\begin{array}{l}\text { TSD unit is undergoing } \\
\text { closure. Waste treated and } \\
\text { disposed of or now in storage } \\
\text { at the CWC. }\end{array}$ \\
\hline
\end{tabular}

$$
\begin{array}{ll}
\text { CWC } & =\text { Central Waste Complex } \\
\text { DST } & =\text { Ddouble-shell tank } \\
\text { PUREX } & =\text { Plutonium-Uranium Extraction } \\
\text { TSD } & =\text { Treatment, storage, and/or disposal }
\end{array}
$$


DOE/RL-97-14

This page intentionally left blank.

T1-2.2 


\subsection{SITE SUMMARY}

This section summarizes the generation, characterization, storage, treatment, and reduction of radioactive LDR waste at the Hanford Facility. It also discusses the variances, surface impoundment exemptions, treatment equivalency petitions, and time extensions required to manage this waste within the requirements established by the Tri-Party Agreement, FFCAct, and 40 CFR 268.

\subsection{WASTE GENERATION}

The projected volumes of mixed waste to be generated are shown in Table 2-1. The assumptions governing these generation rates are discussed in Chapter 1.0, Section 1.2. These assumptions are summarized in the following list.

- The operation of waste pretreatment, treatment, and disposal units will proceed as scheduled in the Tri-Party Agreement (Ecology et al. 1989).

- It is assumed that obligations of DOE arising under the Tri-Party Agreement will be fully funded. The DOE will take all necessary steps to obtain timely funding to meet its obligations under the Tri-Party Agreement. Ecology and EPA will assist RL in determining the specific tasks required to support the corresponding negotiated work schedule for each fiscal year, but will not become involved with the internal DOE budget process.

The annual waste generation volumes presented in Table 2-1 represent the current best estimates of future waste generation for each of the LDR mixed waste streams or the quantity of mixed waste that will be added to TSD units. These estimates are based on detailed evaluation of plant operating schedules, past operating history, and projections of future waste generation. The projected generation volumes may be higher or lower than the actual generation rates because of changes in waste treatment or production schedules or waste minimization activities.

I Decommissioning and remediation activities for RCRA past-practice units are anticipated to generate large volumes of contaminated soils and debris (e.g., contaminated structures, drums, tanks, piping, equipment, and cleanup debris) some of which may be subject to regulation under the LDR Program. Volumes for these RCRA activities are estimated until they are delineated in RCRA permit modifications and Decontamination and Decommissioning Work Plans. No estimates are available for this waste at this time, and thus projected volumes for RCRA pastpractice waste are not included in this report. Additionally, volumes for CERCLA onsite waste activities are typically documented in records of decision and action memoranda and are not included in this RCRA LDR report. Alternative treatment standards for debris were promulgated by EPA on August 18, 1992 (57 FR 37194). These may be used to satisfy LDR requirements in lieu of treating debris to the treatment standards for the waste codes for which the debris has been designated hazardous. Specific treatment standards for LDR soils have not been promulgated as 
by EPA on August 18, 1992 (57 FR 37194). These may be used to satisfy LDR requirements in lieu of treating debris to the treatment standards for the waste codes for which the debris has been designated hazardous. Specific treatment standards for LDR soils have not been promulgated as I of March 1997. However, on promulgation of these standards, treatment, and possibly expanded storage capacity, for waste generated by decommissioning and remediation activities will require planning and development. Should promulgated standards not be feasible for these soils and debris, variances from such standards or contained-in determinations may be applied for. Extended storage of this waste would be allowable pursuant to Tri-Party Agreement provisions dealing with LDR waste. Planning information, as it develops for this waste, will be incorporated into future revisions of this report.

\subsection{WASTE CHARACTERIZATION}

Mixed waste at the Hanford Site has been characterized, as documented in this plan, based on current process knowledge and, where available, waste sample analyses. Sampling and characterization of waste will continue as necessary until the waste is disposed of. Future characterization plans for the waste are summarized in Table 2-2. Individual waste streams are described in Chapter 3.0.

The dangerous waste designations for the various waste streams/storage facilities are summarized in Table 2-3. This table indicates the types of waste that are currently stored. In some cases, additional waste codes are included for waste that may be received in the future. Dangerous waste, as defined by Washington State regulations, encompasses more waste types than are covered under the federal hazardous waste program. Therefore, in some cases, the waste designations will include federal and state-only waste codes based on the best available information. Future waste characterization and complete implementation of the WAC 173-303 amendments may show that additional or fewer waste codes are applicable to a waste stream. Any changes resulting from future characterization will be included in updates to this report. Washington State-only information is included in this report for completeness only because stateonly LDRs are not subject to the RCRA statutory requirements.

The waste stored in the SSTs and DSTs, and the silver nitrate waste stored in PUREX Tunnels 1 and 2 have been assigned the D001 (ignitable) waste designation because of the presence of oxidizers, nitrates, and/or nitrites. They are not ignitable by themselves, and the designation results from the possibility of them reacting with other materials.

The F001 through F005 waste codes (spent halogenated and nonhalogenated solvents) have been assigned to the SST- and DST-system waste, not because the waste contains significant quantities of spent solvents, but because of the mixture rule from the introduction of spent solvents. Additionally, these waste codes apply to all downstream waste derived from DST waste, such as process condensate from the 242-A Evaporator. The past discharges of spent solvents to SSTs and DSTs and resultant tank-to-tank transfers have mixed essentially all of the waste in the tanks. This has resulted in all of the SST and DST waste and downstream waste from these tanks being designated F001 through F005. The tank waste does not contain large 
The F039 waste code was added to the DST, LERF, 242-A Evaporator, Low-Level Burial Ground, CWC, WRAP Module 1 and other Hanford Facility TSD units' Part A Form 3 Permit applications in November 1994 to allow for future generation of waste potentially listed with this code from onsite mixed waste disposal operations. The 200 Area ETF can delist F039 waste streams if they are only derived from F001 through F005 sources. Currently, no F039 waste is being generated or stored at the Hanford Facility.

Washington State promulgated new régulations that affect designation of waste generated and waste in storage after November 20, 1995 [WAC 173-303-070(3)(a) and 173-303-070(5)]. Federal waste codes (e.g., D001) are assigned to a waste first, followed by state codes (e.g., WT01). The state code is not assigned in many cases based on these amendments. (The new regulations have not been applied to waste in storage that was generated before the effective date. The new regulations will be applied to stored waste that is moved to a new storage location after the effective date.)

The schedule and means for reporting waste characterization data are outlined in the Tri-Party Agreement (Ecology et al. 1989) as amended by Section 9.6, "Data Reporting Requirements." This section states that DOE will make available to Ecology and EPA all validated laboratory analytical data collected pursuant to the Tri-Party Agreement within 15 work days of data validation. Within 1 week after the laboratory data are validated, DOE will notify Ecology and EPA of their availability in the Hanford Environmental Information System. This notification will include the time and location of the sampling, the type of data available, and a list of the sample parameters or target compounds. The time limits for reporting sample analyses are 216 days for SST analyses, 176 days for hot cell analyses, and 126 days for low-level and mixed waste. (All time limits are computed from the date of sampling.)

\subsection{WASTE STORAGE}

The Hanford Site has 13 streams, as defined by this report, that currently are stored in TSD units. These streams can be divided into two groups. The first group contains five streams that are no longer actively receiving waste. These streams are SST waste, suspect-TRU, TRU waste, and other regulated low-level burial ground waste, the PUREX containment building, 324 Building $\mathrm{HLV}$, and B Plant organic waste. The second group is eight streams that are currently receiving or could receive waste for storage to await treatment and disposal. These streams are DST waste, the LERF for 242-A Evaporator Process Condensate and other aqueous wastes, PUREX Storage Tunnels 1 and 2, the CWC, the 224-T Transuranic Waste Storage and Assay Facility (TRUSAF), 324 Building REC, B Plant Cell 4 waste, and B Plant containment building storage. 224-T TRUSAF is scheduled to be emptied before August 31, 1997. Its inventory will be transferred to the CWC. The key characteristics of these waste streams are summarized in Table $2-4$.

The storage unit capacity for solid mixed waste at the Hanford Site is projected to be adequate for all currently generated mixed waste until at least 2001 , assuming the availability of three additional storage facilities as part of the Central Waste Complex (CWC). This projection is 
based on the individual projections of all generators who ship waste to the $\mathrm{CWC}$ and the availability of planned storage and treatment facilities. The projection of waste generation rates is refined annually. Should future projections indicate that increased storage capacity is required, additional storage units will be constructed and permitted as needed.

The LERF provides interim storage and treatment (through flow and $\mathrm{pH}$ equalization) for some aqueous waste streams that will be treated at the 200 Area ETF. The 200 Area ETF provides treatment and storage for aqueous waste such as the process condensate from the 242-A Evaporator. A treatment exemption has been granted in accordance with 40 CFR 268.4 that allows use of LERF as a storage and treatment unit with certain restrictions.

By 1998 , the currently available DSTs will essentially be filled to capacity, using current space projections. The baseline plans are to consolidate and evaporate DST waste, which will eliminate the need to provide additional tank space. This will allow for their continued use for waste storage until after 1998. Change request M-42-95-01, which changes the Tri-Party Agreement Milestone M-42-00 (providing for additional DST capacity) due date to "TBD" and deletes all supporting uncompleted M-42 interim milestones, was approved December 1, 1995. This change request postpones the construction of new DSTs indefinitely.

Except for the SST system and the 183-H Solar Evaporation Basins, the storage units for mixed waste at the Hanford Site have not released any dangerous constituents to the environment. This has been determined through all available information such as monitoring data, inspections, 1 and operational history. The SSTs have released an estimated 3,148 cubic meters of liquid waste to the ground. This estimate excludes any cooling water added to tanks after they were known to be leaking. To minimize further releases from this storage unit, the pumpable liquid portion of the waste stored in the SSTs is being transferred to the DSTs. Although the amount of hazardous constituents released from the 183-H Solar Basins has not been estimated, the data evaluation I report for this unit characterized the soil contamination associated with the release. The basins

are no longer covered in this report because the waste has been transferred to the CWC.

I

The latest Part B Permit application or closure plan submittal date for each mixed waste storage unit is shown in Table 2-4.

The general characteristics of the radioactive mixed waste currently in storage at the Hanford Site are summarized in Table 2-5. The table shows that as of December 31, 1996, the Hanford Site stores approximately 226,700 cubic meters of radioactive mixed waste. The bulk of this waste ( 98.9 percent) is stored in the SSTs (60.2 percent) DSTs ( 31.9 percent), LERF I ( 2.3 percent), and CWC ( 4.5 percent). The table also indicates how much waste is LLW, TRU waste, or HLW.

\subsection{WASTE TREATMENT}

The LDRs apply to each type of hazardous waste that has been restricted from land disposal. Treatment standards are identified in two different ways: as concentration-based 
standards and as technology-based standards. Concentration-based standards have been developed based on "best demonstrated available technology." Treatment to meet concentrationbased standards can be pursued via any technology (other than dilution, which is not permissible) except for cyanides, which must be destroyed; the only requirement is that the waste be treated to reduce the concentration(s) of the constituent(s) of concern. However, waste types to which technology-based standards apply must be treated via the pertinent specified technology. Hazardous waste that carries multiple RCRA codes must be treated pursuant to the standards for each waste code (and subcategory, when applicable) unless 40 CFR 268.9(a) and (b) applies. In situations where overlap occurs, the more stringent standard must be applied. One of EPA's recent LDR rulemaking efforts (59 FR 47982) resulted in the Universal Treatment Standards (UTS). The UTS contain numerical limits for underlying hazardous constituents. The UTS were originally applicable to certain ignitable (D001)-, corrosive (D002)-, and toxic-characteristic organic (D018-D043) hazardous waste and to pesticide (D012-D017) nonwastewaters that are destined for land disposal. Phase III LDR regulations issued in 1996 (61 FR series) added reactive (D003) waste. 62 FR 7502, issued in 1997, updated the UTS table of 40 CFR 268.48 by consolidating previous changes.

This plan summarizes the treatment standards applicable and those proposed for Hanford I Site waste; discussions of the following waste streams are included:

- DST Waste (Chapter 3.0, Section 3.1)

- SST Waste (Chapter 3.0, Section 3.2)

- 242-A Evaporator Process Condensate (Chapter 3.0, Section 3.3)

- PUREX Storage Tunneis Waste (Chapter 3.0, Section 3.4)

- PUREX Containment Building Storage (Chapter 3.0, Section 3.5)

- CWC Stored LLW, TRU Waste, and PCB Waste (Chapter 3.0, Section 3.6)

- Suspect-TRU and TRU Waste and Other Regulated Low-Level Burial Ground Waste (Chapter 3.0, Section 3.7)

- 224-T Transuranic Waste Storage and Assay Facility (TRUSAF) Stored Waste (Chapter 3.0, Section 3.8)

- $\quad 324$ REC Waste (Chapter 3.0, Section 3.9)

- $324 \mathrm{HLV}$ Waste (Chapter 3.0, Section 3.10)

- B Plant Cell 4 Waste (Chapter 3.0, Section 3.11)

- B Plant Containment Building Storage (Chapter 3.0, Section 3.12) 
The applicable treatment standards (required by 40 CFR 268 and WAC 173-303-140) and the proposed treatments for the Hanford Site mixed waste are summarized in Table 2-6. All of the contributing streams to the DST system are combined as one because all will be pretreated into HLW and LLW streams and vitrified similarly (Table 2-6). The schedule for the operation of the treatment units is provided in Figure 2-1.

Applicable treatment alternatives are described in Chapter 3.0. The use of offsite commercial treatment technologies is currently under consideration for some waste streams. The use of onsite commercial technologies is also possible. The DOE is considering the use of nontraditional contracting approaches for site remediation work, i.e., "privatization." The use of commercial technologies is likely to play a role in site remediation work (primarily under CERCLA regulations). Certain solid waste treatment operations, such as stabilization and thermal treatment and vitrification of SST and DST waste, are planned to be privatized.

The Tri-Party Agreement specifies the required dates for construction and startup of, and waste treatment in, the major treatment facilities. There are no requirements for accelerated treatment beyond these dates. All of this waste is considered to be stored in an environmentally sound manner with the exception of SST waste and some DSTs with waste having unique safety problems because of chemical and/or radiological content. Further details on accelerated treatment are located in the individual waste stream treatment discussions in Chapter 3.0.

\subsubsection{Double-Shell Tank Waste}

The DST waste consists of LLW, TRU waste, and HLW. In the interim storage mode, however, the waste is managed as HLW and is evaporated at the 242-A Evaporator to reduce the tank waste volume. Before treatment for disposal, the waste will be separated (i.e., pretreated) into two streams: a LLW stream and a HLW/TRU stream. The HLW stream may undergo additional treatment as necessary to further reduce its volume and concentrate its radionuclide loading.

Before disposal, appropriate testing of the LLW and HLW/TRU products will be conducted to ensure that the waste will comply with the LDR standards. The HLW subsequently will be disposed of at a HLW repository in a still-to-be- determined national location; the TRU waste is being evaluated for disposal at WIPP near Carlsbad, New Mexico; the LLW will be 1 disposed of near surface at the Hanford Site. Figure 2-2 depicts the DST separation, treatment, and disposal processes.

Several TSD units are planned to perform treatment and disposal processes. A pretreatment facility will be constructed to perform the necessary waste separations, with startup scheduled for December 2002 for the LLW stream and December 2009 for the HLW/TRU waste immobilization facility. Subsequent disposal of treated HLW will begin when a 
I national repository is available. The schedule for these treatment processes is shown in Figure 2-1.

\section{| 2.4.2 Single-Shell Tank Waste}

The SST waste consists of LLW, TRU waste, and HLW; however, in the interim storage mode it is managed as HLW. The physical forms of SST waste are sludge, salt cake, and liquid. Liquid waste, which includes supernatant and interstitial liquid within the salt cake, is being transferred to DSTs for subsequent treatment (as long as the safety status of the SSTs is not changed after pumping). The planning base for SSTs is to retrieve all the waste and transfer it to DSTs where it will then be separated into LLW and HLW/TRU waste fractions (via pretreatment). Both waste fractions will be vitrified for disposal in the same way as the DST waste shown in Figure 2-2. Waste has not been introduced into the SST system since 1980; no additional waste will be generated and stored in the SST system.

\subsubsection{2-A Evaporator Process Condensate}

The DSTs have limited storage space available to support remediation of the tank waste and cleanup of the Hanford Site. Dilute tank waste is concentrated in the 242-A Evaporator to produce a concentrated waste that is returned to the tanks and a process condensate stream. The DSTs contain listed waste as defined by RCRA. The process condensate is a listed waste via the "derived from" rule. The process condensate and other waste water may be stored in the LERF while awaiting treatment in the 200 Area ETF. The ETF treatment system reduces the concentration of radioactive and hazardous waste constituents in the waste water to acceptable levels using what is currently best available technology. The treated effluent is discharged to a state-approved land disposal site. A delisting petition was approved by the EPA (60 FR 31115). The petition exempts the treated effluent from the requirements of the hazardous waste regulations under RCRA and imposes certain effluent quality restrictions. Because the treated effluent is no longer subject to the hazardous waste regulations, the LDRs do not apply to the effluent stream.

\subsubsection{PUREX Storage Tunnels 1 and 2 and PUREX Containment Building $W$ aste}

The PUREX Plant waste includes lead, mercury, silver, cadmium, chromium, barium, and absorbed mineral oil waste stored in the PUREX tunnels and chromium solid waste stored in the PUREX Containment Building. The specified treatment for radioactive lead solids not used as shielding is macroencapsulation. The required treatment for mercury waste is amalgamation or retorting and recovery. Any treatment that achieves the constituent concentration limits of less than 5.0 milligrams per liter is acceptable for the silver waste. Any treatment that achieves the constituent concentration limits of 1.0,5.0, and 100, milligrams per liter are acceptable for cadmium, chromium, and barium waste, respectively, in accordance with 40 CFR 268.40. 


\subsubsection{Central Waste Complex Stored Low-Level, Transuranic, and Polychlorinated Biphenyl Waste; 224-T TRUSAF Stored Waste; and Suspect-Transuranic, Transuranic, and Other Regulated LLBG Waste}

\section{।} I various treatment units.

The Hanford Site's CWC and 224-T Building (TRUSAF) are storage units for storing lowlevel mixed waste (LLMW), and transuranic mixed waste (TRUM). Some of the waste is cocontaminated with PCBs. The CWC functions as a waste warehouse supplying feed to the

The Waste Receiving and Processing Module 1 (WRAP 1) will certify all the contacthandled (CH) TRU/TRUM waste for disposal at the WIPP facility. Non-mixed waste (LLW) will be disposed of in near-surface disposal units located on the Hanford Site. The CH-LLMW will be treated as necessary to meet RCRA and State LDR requirements. LLMW will be treated using both onsite treatment capabilities and offsite commercial facilities. Remote-handled $(\mathrm{RH})$ waste will be put into interim storage until processing capabilities are available.

Suspect-TRU waste excavated from the near-surface storage units will be assayed and separated into TRU, TRUM, and LLW streams at the WRAP 1 facility. Suspect-TRU waste is primarily contained in 208-L (55-gal) drums, metal boxes, wood boxes, and fiberglass-reinforced plywood boxes. These containers are located in several near-surface storage units in the lowlevel burial grounds (LLBG). Figure 2-3 depicts the LLMW, TRU/TRUM, and LLW disposition functional flow. TRUSAF is scheduled to be emptied in FY 1997; the contents will be transferred to the CWC.

\section{| 2.4.6 324 REC Waste}

The 324 REC waste has accumulated during research activities over a period of years. The waste consists of contaminated equipment, construction materials, and evaporated liquids that I have accrued on the floor of the REC B-Cell. In addition, particulate materials introduced with normal air flow into the cell became contaminated. Cleanout of the hot cells was initiated in 1988 with completion expected by 2000 . At the end of fiscal year 1995 , more than 74 percent of the floor area had been cleared of potentially dispersible mixed waste. This dispersible waste was consolidated and containerized within B-Cell. All containerized mixed waste has been shipped to permitted storage at the PUREX storage tunnels. Treatment alternatives are currently being evaluated for all except the 0.5 cubic meter of contact-handled mixed waste lead solids that was shipped to the CWC. The REC also contains the solid residues from the treatment of the $324 \mathrm{HLV}$ waste. 


\subsubsection{HLV Waste}

The $324 \mathrm{HLV}$ waste consists of high-activity radioactive solutions, which are no longer needed for research and development activities. These solutions were designated as mixed waste in FY 1994. An Ecology-supported treatment alternative is being pursued to reduce the radioactivity to contact-handled limits that would allow the waste to be consolidated with other Hanford Facility waste and recover strontium for use in medical isotopes. The waste solutions were transferred to D-Cell in the REC and, after treatment, the remaining mixed waste residues were transferred to B-Cell (approximately 0.6 cubic meters).

\subsubsection{B Plant Cell 4 Waste}

The mixed waste in B Plant's Cell 4 consists of lead solder on incandescent lamp bulbs that were changed in the hot cells of the WESF Facility. The specified LDR treatment of radioactive lead solder is macroencapsulation. B Plant received official notification to deactivate the facility in October 1995. B Plant Cell 4 waste will remain in place following deactivation. A Preclosure Work Plan that addresses this situation will be submitted to Ecology. Limited quantities of Cell 4 waste may continue to be generated during the ongoing deactivation activities.

\subsubsection{B Plant Containment Building Storage}

Waste stored in the B Plant Containment Building includes failed process jumpers and equipment containing lead that was used as counterweights and/or radiation shielding. This equipment was used to process listed tank waste and may be contaminated with residues of the processed waste. The specified LDR treatment for radioactive lead solids is macroencapsulation. Any treatment that achieves a constituent concentration level of 5.0 milligrams per liter for the silver waste is acceptable. Hazardous debris treatment in the permitted containment building that meets the standards specified in 40 CFR 268.45 is also acceptable. B Plant received official notification to deactivate the facility in October 1995. B Plant Containment Building Storage Waste will remain in place following deactivation of the facility. A Preciosure Work Plan that addresses this situation will be submitted to Ecology. Additional B Plant Containment Building waste may be generated during B Plant deactivation activities.

\subsubsection{B Plant Organic Waste}

The B Plant Organic Waste is what remains of the organic solvents used in the strontium solvent extraction process performed in B Plant from 1968 to 1979. Since then, the waste has been stored in canyon process tanks. The waste consists of the following solvents; di(2-ethylhexyl) phosphoric acid (D2EHP), tributyl phosphate, and normal paraffin hydrocarbons. Estimates from process knowledge conclude that the waste matrix is 20 percent, 10 percent, and 70 percent of the three chemicals, respectively. Attempts made in 1989 and 1992 to characterize the waste were unsuccessful; however the waste is assumed to contain F-listed constituents. Any 
treatment used on this waste will need to meet the standards found in 40 CFR 268.40. An extensive characterization effort is scheduled for 1997 as part of the effort to transfer the organic waste to storage tanks outside of the B Plant facility. This transfer is expected to take place by the end of February 1997. Various disposal and treatment methods are being considered for final disposition, including solidification and incineration. No decisions have been reached. No additional B Plant organic waste will be generated.

\subsection{WASTE MINIMIZATION}

\subsubsection{Waste Minimization Program Elements}

Six basic elements make up the overall waste minimization program: top management support, characterization of waste generated and the process that generates it, waste minimization assessments, cost allocation, technology transfer, and program evaluation.

2.5.1.1 Statement of Management Support/Commitment. The RL Manager and contractor management are committed to minimizing the generation of waste by giving preference to source reduction, material substitution, and environmentally sound recycling over treatment, storage, and disposal of such waste. Management takes appropriate action to provide adequate personnel, budget, training, and resources on a continuing basis to ensure that the objectives of the waste minimization program are met.

Annual goals have been established by both $R L$ and contractor management for all types of waste generated at the Hanford Site. Through the performance of waste minimization assessments and selection of economically practicable options, the site goals are translated into specific goals for each facility.

Management support is further evidenced by including waste minimization training in the Hanford General Employee Training program, through incentive programs that reward individual and group contributions, and by including waste minimization in job performance evaluations of persons having waste minimization responsibilities.

2.5.1.2 Characterization of Waste Generation. Waste that is generated is characterized to obtain information on quantity generated, hazardous constituents, and their concentration.

2.5.1.3 Periodic Waste Minimization Assessments. Waste minimization is to be integrated into the design of any new facility or the modification of an existing facility or process. Waste that is nonetheless generated will periodically be assessed for waste minimization potential through pollution prevention opportunity assessments. This methodology requires that a pollution prevention opportunity assessment team be formed to evaluate each waste-generating process selected. 
2.5.1.4 Cost Allocation System. A cost accounting system that accounts for the "true cost" of waste that is generated by the facility must include short- and long-term costs arising from underutilization of raw materials found in the waste stream, management of the waste generated, waste disposal, and third-party liabilities if the waste is improperly disposed of. Associated costs will include personnel, record keeping, transportation, pollution control, equipment, treatment, storage, disposal, liability, compliance, and oversight costs.

2.5.1.5 Technology Transfer. The transfer of federally developed technology between laboratories and potential users is a contractual responsibility of DOE facilities and laboratories. Activities involving technology transfer must be coordinated through the contractor's office that has been designated to represent the facility on the Federal Laboratory Consortium for Technology Transfer. The Federal Laboratory Consortium promotes technology transfer through links to the public and private sectors and through support services such as training and assistance in implementing partnership opportunities. Transfer of technologies specific to waste minimization may develop from information exchange systems, workshops, or topical conferences.

2.5.1.6 Program Evaluation. Achievements and milestones in the program will be a part of the contractor's performance evaluation and determination of award fees. The results of this evaluation by the contractor are reported by the Pollution Prevention group of the integrating contractor to $R L$ in periodic reports.

The following success criteria are available to aid in the demonstration of effective waste minimization efforts:

- Reduced amount of hazardous waste

- Reduced amount of all waste

- Reduced waste management costs

- Improved regulatory compliance

- Reduced health risks

- Increased production efficiency

- Reduced accident risk

- Improved public relations.

\subsubsection{Program Objectives}

The objectives of the waste minimization program are as follows:

- Foster a philosophy to conserve resources and minimize waste and pollution while achieving Hanford Site strategic objectives.

- Promote the use of nonhazardous materials in operations to minimize the potential risks to human health and the environment. 
- Reduce or eliminate the generation of waste through input substitution, process modification, improved housekeeping, and closed-loop recycling to achieve minimal adverse effects to the air, water, and land.

- Comply with federal and state regulations and DOE requirements for waste minimization, waste reduction, and pollution prevention.

- Characterize waste streams and develop a baseline of waste-generation data.

- Identify and implement methods and technologies for waste minimization.

- Target policies, procedures, or practices that may be barriers to waste minimization.

- Enhance communication of waste minimization objectives, goals, and ideas.

- Promote integration and coordination of waste generators and waste managers on waste minimization matters.

- Develop specific goals and schedules for waste minimization activities.

- Create incentives for waste minimization.

- Collect and exchange waste minimization information through technology transfer, outreach, and educational networks.

- Develop mechanisms for fully disseminating current technical information to Hanford Site users.

\subsubsection{Facility-Specific Waste Minimization}

All facilities that generate waste are required to have a waste minimization program in place. The effectiveness and implementation of the programs are audited regularly. The following are key components of the program.

- To the extent practical, all mixed waste is segregated and packaged separately from LLW or TRU Waste that contains no hazardous or dangerous constituents.

- The volume of solid mixed waste is reduced by compaction when possible. The volume of liquid mixed waste is reduced by removing or destroying contaminants in the waste water.

- To minimize the generation of mixed waste, generators actively seek nondangerous alternatives for the dangerous constituents in their processes. 
- Waste is characterized and the potential for minimization is investigated.

- Minimization goals are set annually and tracked quarterly.

- Where allowed by regulation, mixed waste is treated to remove the dangerous constituents.

- Where allowed by regulation, corrosive materials are neutralized to remove their corrosive characteristics or are packaged in a manner that ensures the integrity of the containment barriers.

- Waste handling, segregation, and certification will be performed following detailed procedures when the disposal criteria are promulgated.

।

I in place or planned for the waste units addressed in this plan. The table also shows schedules for implementation and the projected effectiveness of the method.

I Dilute tank waste is concentrated in the 242-A Evaporator to produce a concentrated waste 1 that is returned to the tanks and a process condensate stream. The waste volume reduction I achieved is typically 50-80 percent, depending on the initial concentration of chemical constituents i in the tank waste. Some process condensate is recycled within the 242-A Evaporator for uses I such as pump seal water. This minimizes the volume of additional waste generated.

I tituents in waste water to acceptable levels using what is currently best available technology The effective waste volume reduction capability of the ETF is more than 99 percent.

The waste currently stored at the CWC will be processed at one of the WRAP facilities 1 (described in Chapter 3.0, Section 3.6) or in commercial entities before disposal.

In a typical year, waste reduction practices at the Hanford Site will reduce the waste volume by well over 100,000 cubic meters. The majority of the reduction is from treatment.

In addition to specific waste reduction sections in Chapter 3.0, waste reduction at the | Hanford Site is described in the Annual Report of Waste Generation and Pollution Prevention | Progress 1994 (DOE 1994).

\section{| 2.6 VARIANCES, SURFACE IMPOUNDMENT EXEMPTIONS, TREATMENT EQUIVALENCY PETITIONS, AND TIME EXTENSIONS}

Removal and treatment of the Hanford Site stored mixed waste to meet LDR requirements are summarized in Section 2.4 . 
I The Tri-Party Agreement (Ecology et al. 1989) provides the plan and schedule for treatment of Hanford Site mixed waste currently in storage. The FFCAct of 1992 also contains applicable provisions. Refer to Section 1.1 for additional detail.

1 If variances, surface impoundment exemptions, treatment equivalency petitions, or time extensions are required as a result of delays in the development of treatment, storage, or disposal capacity, they will be applied for in accordance with the procedures detailed in the Tri-Party Agreement and/or applicable regulations. Specific variances, exemptions, etc. that have been I granted or applied for are covered with the applicable waste streams.

The Tri-Party Agreement provides for extending a schedule or deadline on receipt of a timely request for extension and when good cause exists for the requested extension. Any request for extension shall be submitted in writing and shall specify the following:

- The timetable and deadline or schedule for which the extension is sought

- The length of the extension sought

- The good cause for the extension

- Any related deadline or schedule that would be affected if the extension were granted.

Good causes for an extension include the following:

- An event of force majeure as defined in Article XLVII of the Tri-Party Agreement, subject to Ecology's reservation in Paragraph 147

- A delay caused by another party's failure to meet any requirement of the Tri-Party Agreement

- A delay caused by invocation of dispute resolution to the extent provided by Paragraph 30(f) and Paragraph 59(I) or judicial order

- A delay caused, or likely to be caused, by an extension granted to another deadline or schedule

- Any other event or series of events mutually agreed to by the parties as constituting good cause. 
DOE/RL-97-14

Figure 2-1. Operating Schedules for Units Managing

Land Disposal Restricted Waste. (sheet 1 of 16)

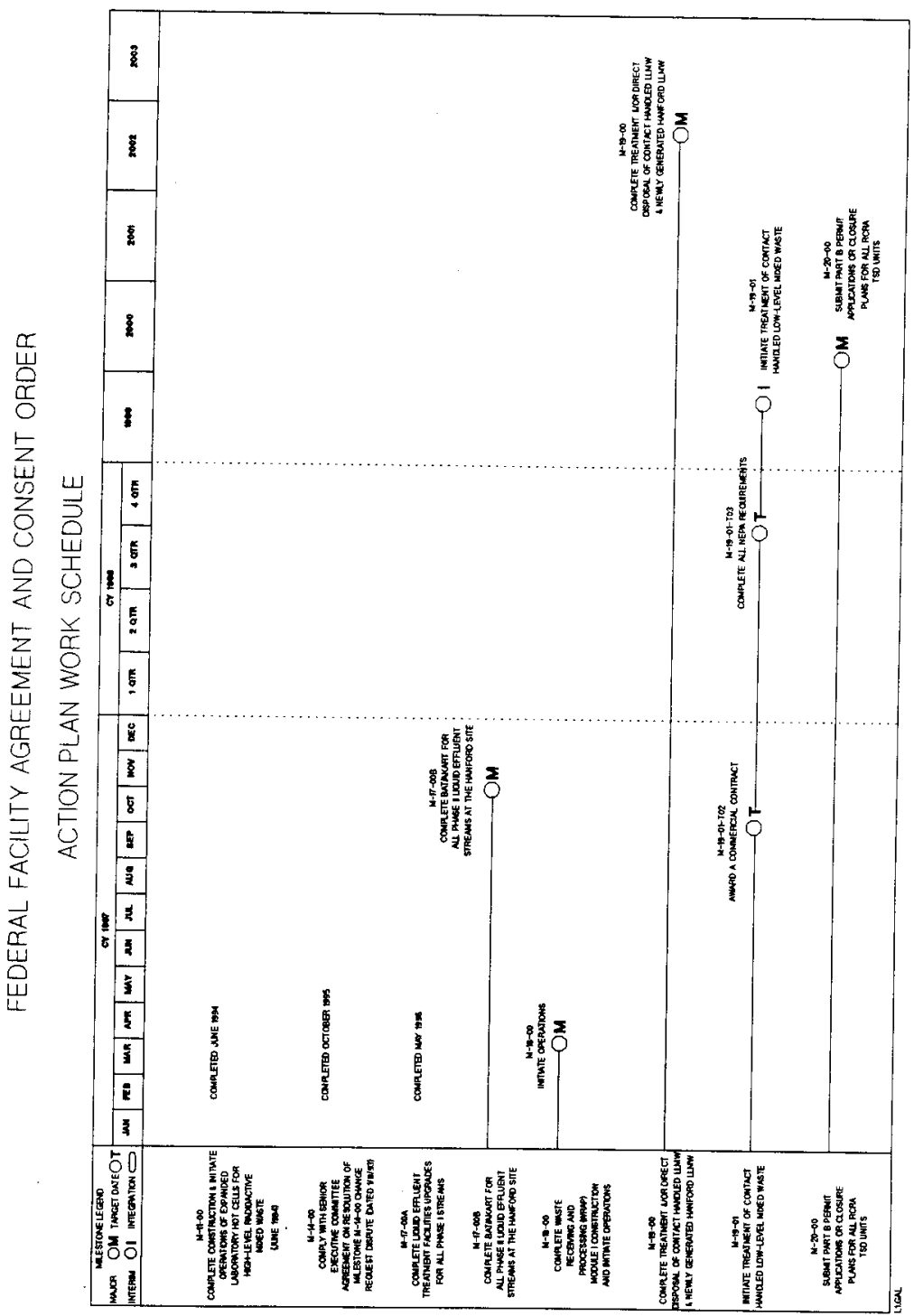

F2-1.1 
Figure 2-1. Operating Schedules for Units Managing Land Disposal Restricted Waste. (sheet 2 of 16)

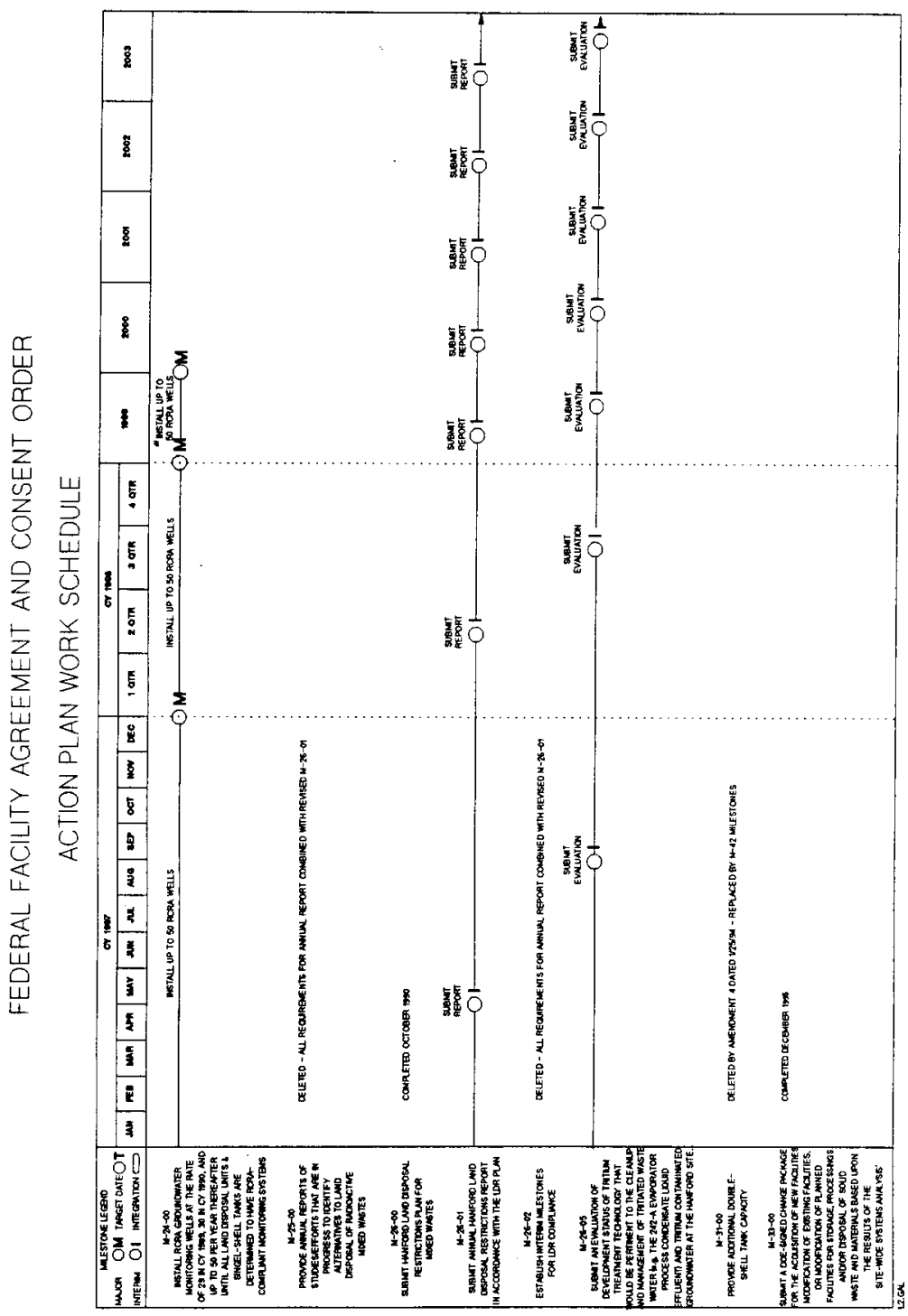

F2-1.2 
Figure 2-1. Operating Schedules for Units Managing Land Disposal Restricted Waste. (sheet 3 of 16)

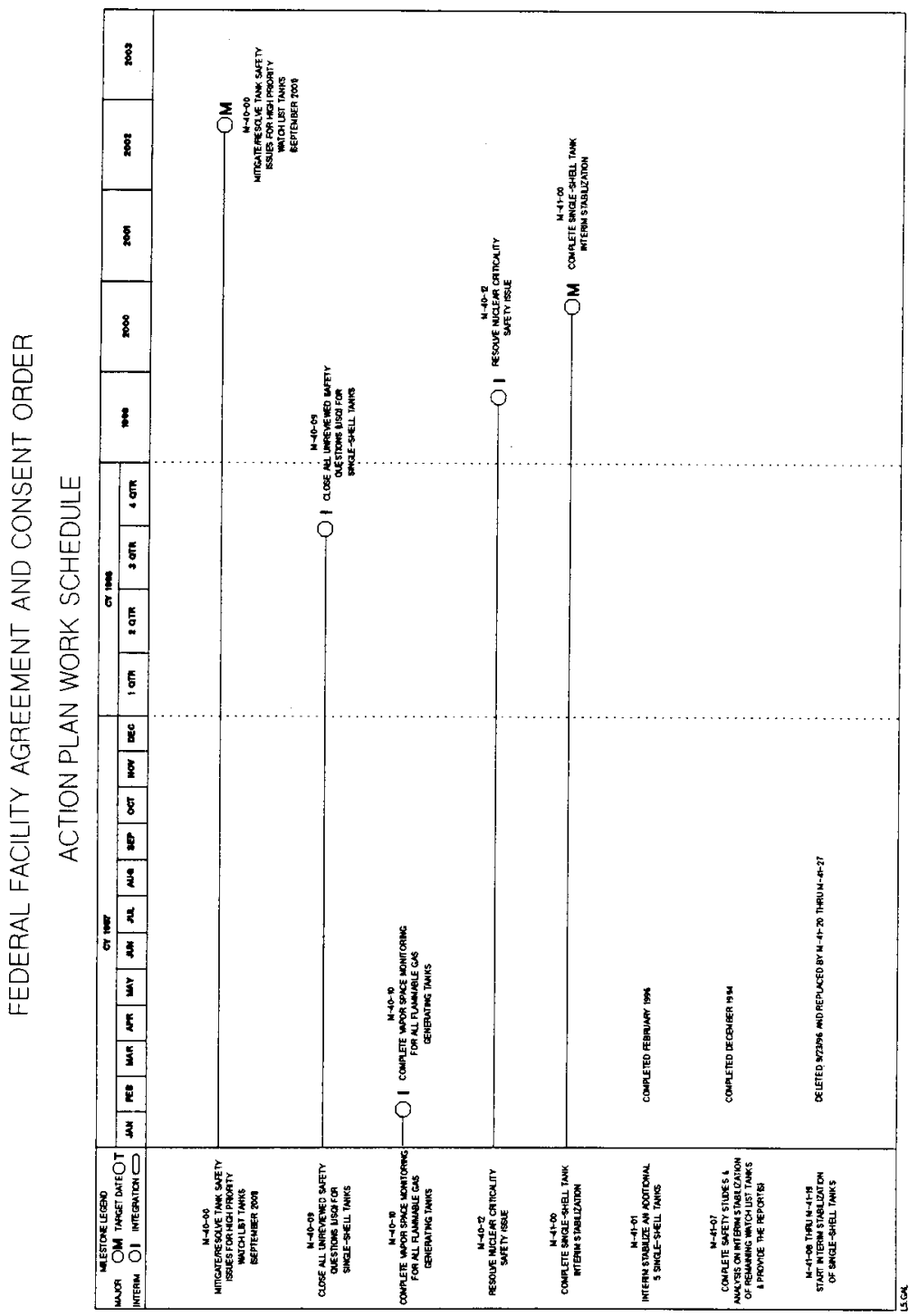

F2-1.3 
FEDERAL FACILITY AGREEMENT AND CONSENT ORDER

ACTION PLAN WORK SCHEDULE

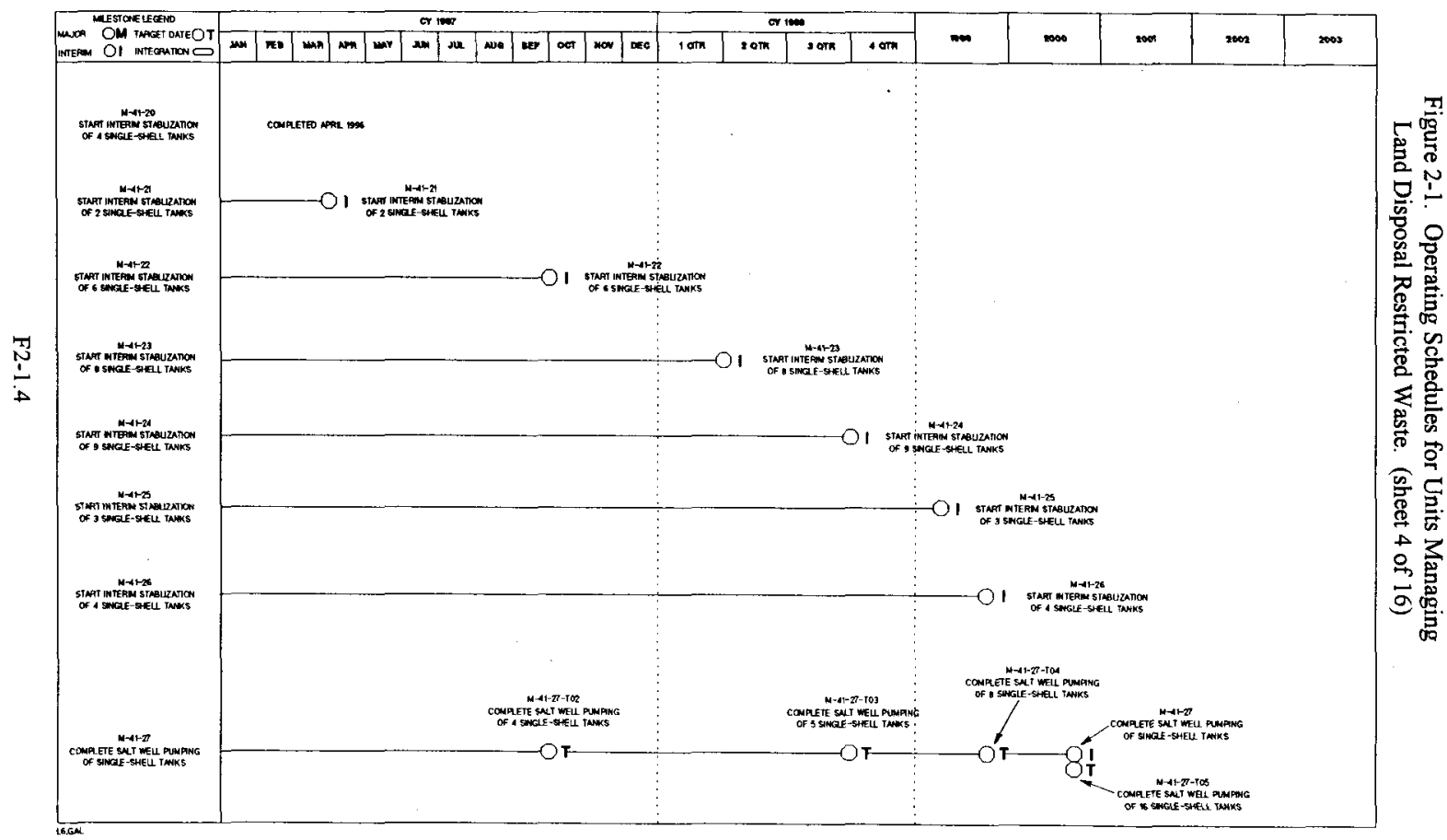


Figure 2-1. Operating Schedules for Units Managing Land Disposal Restricted Waste. (sheet 5 of 16)

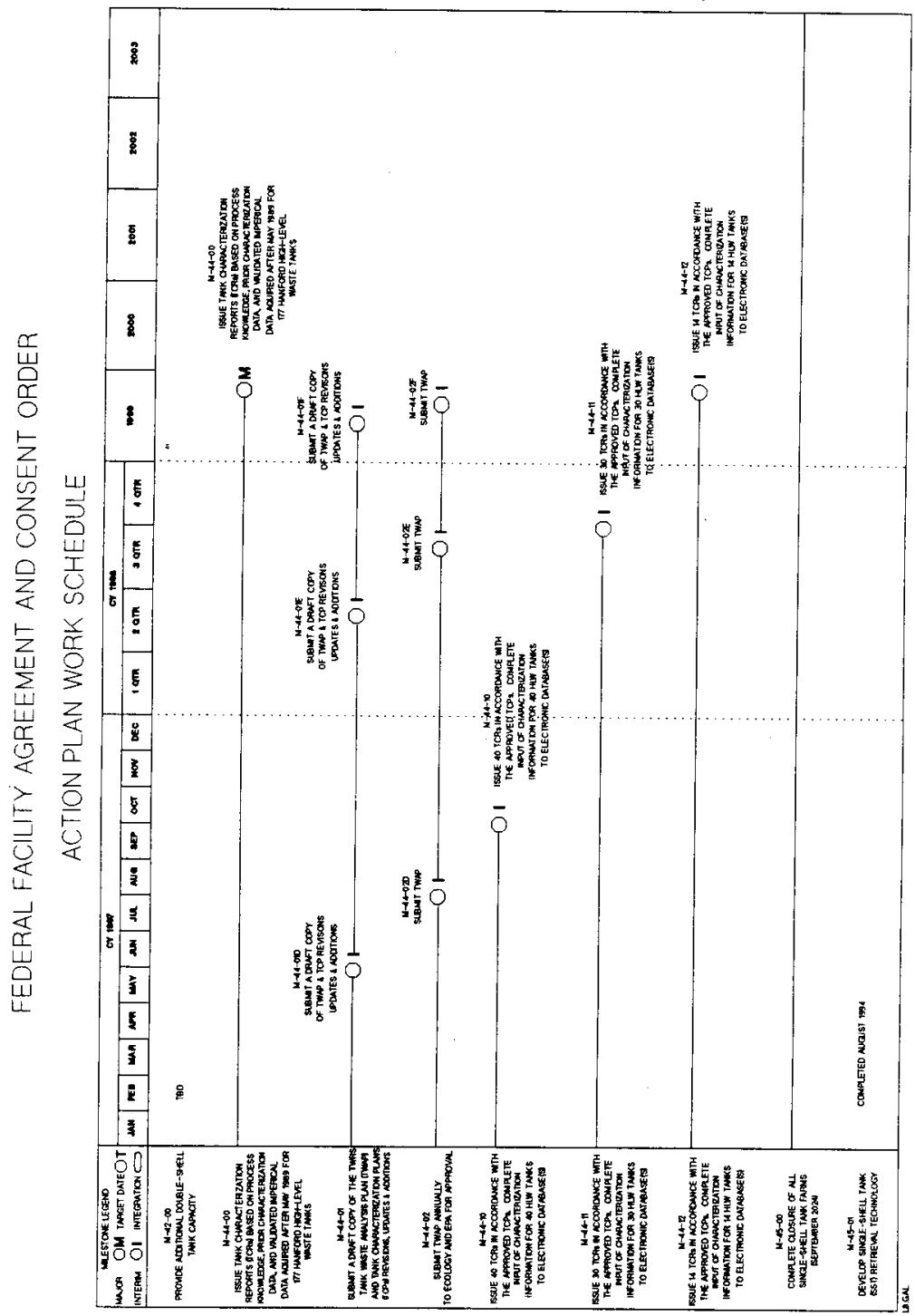

F2-1.5 
Figure 2-1. Operating Schedules for Units Managing Land Disposal Restricted Waste. (sheet 6 of 16)

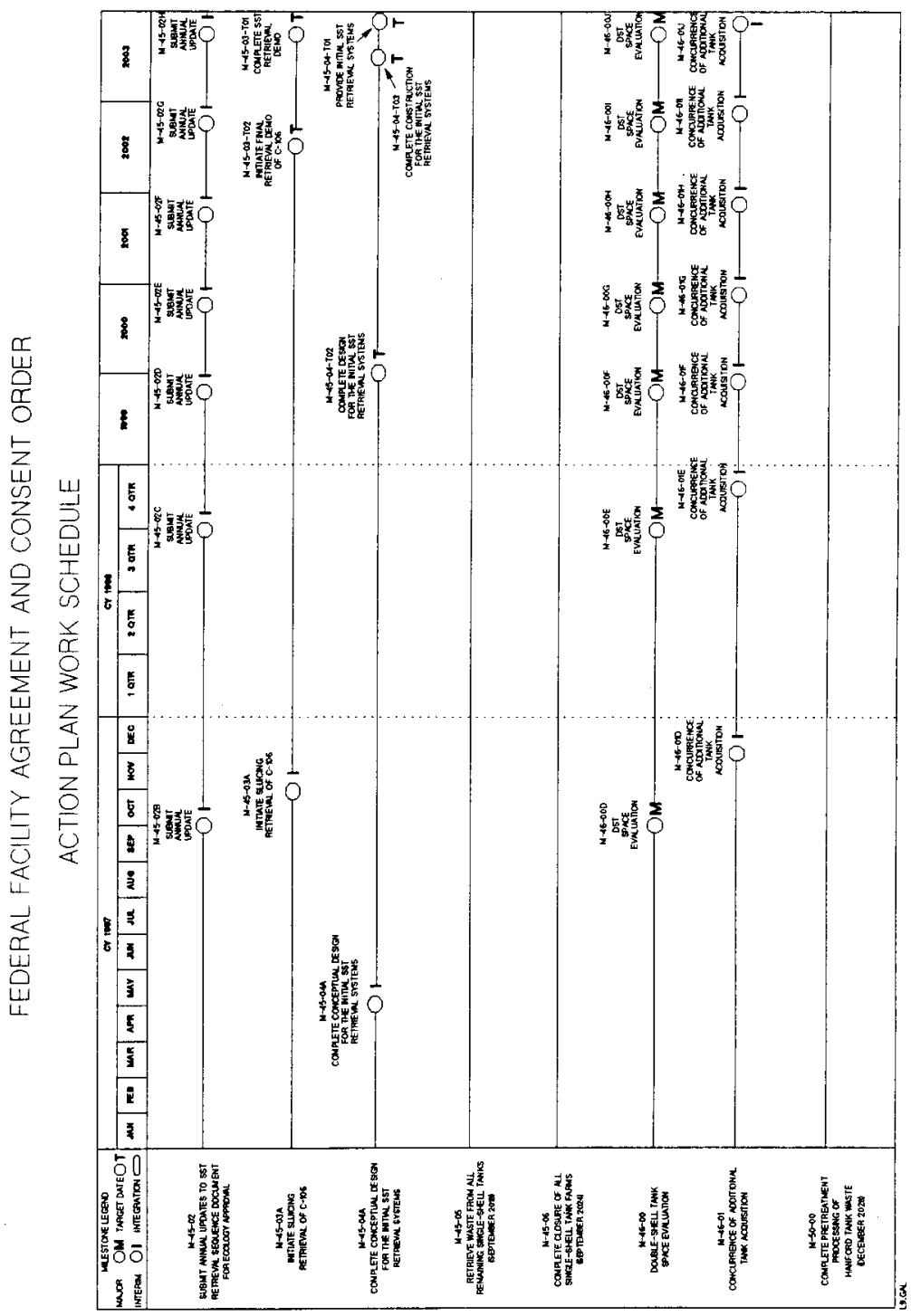

F2-1.6 
Figure 2-1. Operating Schedules for Units Managing Land Disposal Restricted Waste. (sheet 7 of 16)

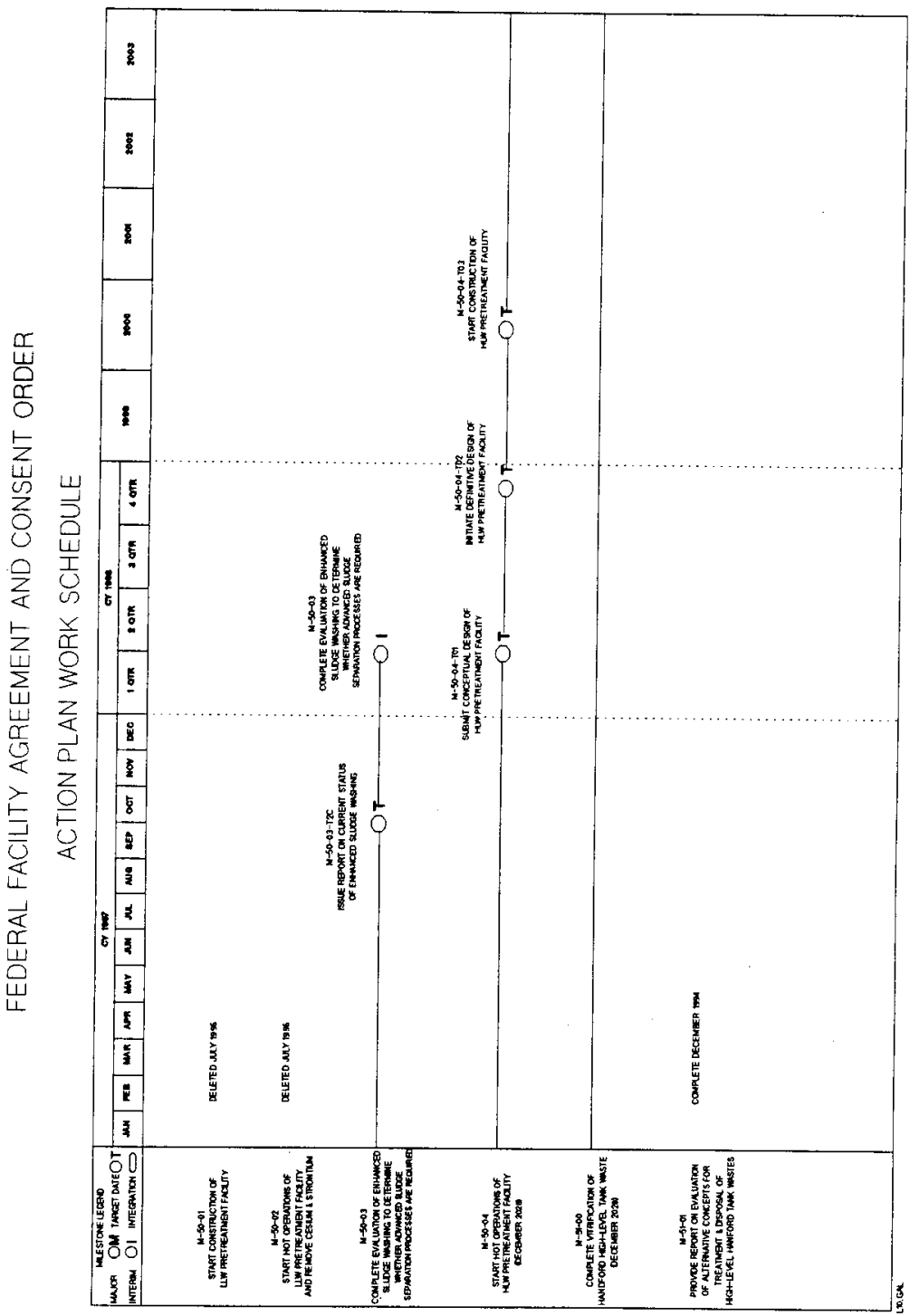

F2-1.7 
Figure 2-1. Operating Schedules for Units Managing Land Disposal Restricted Waste. (sheet 8 of 16)

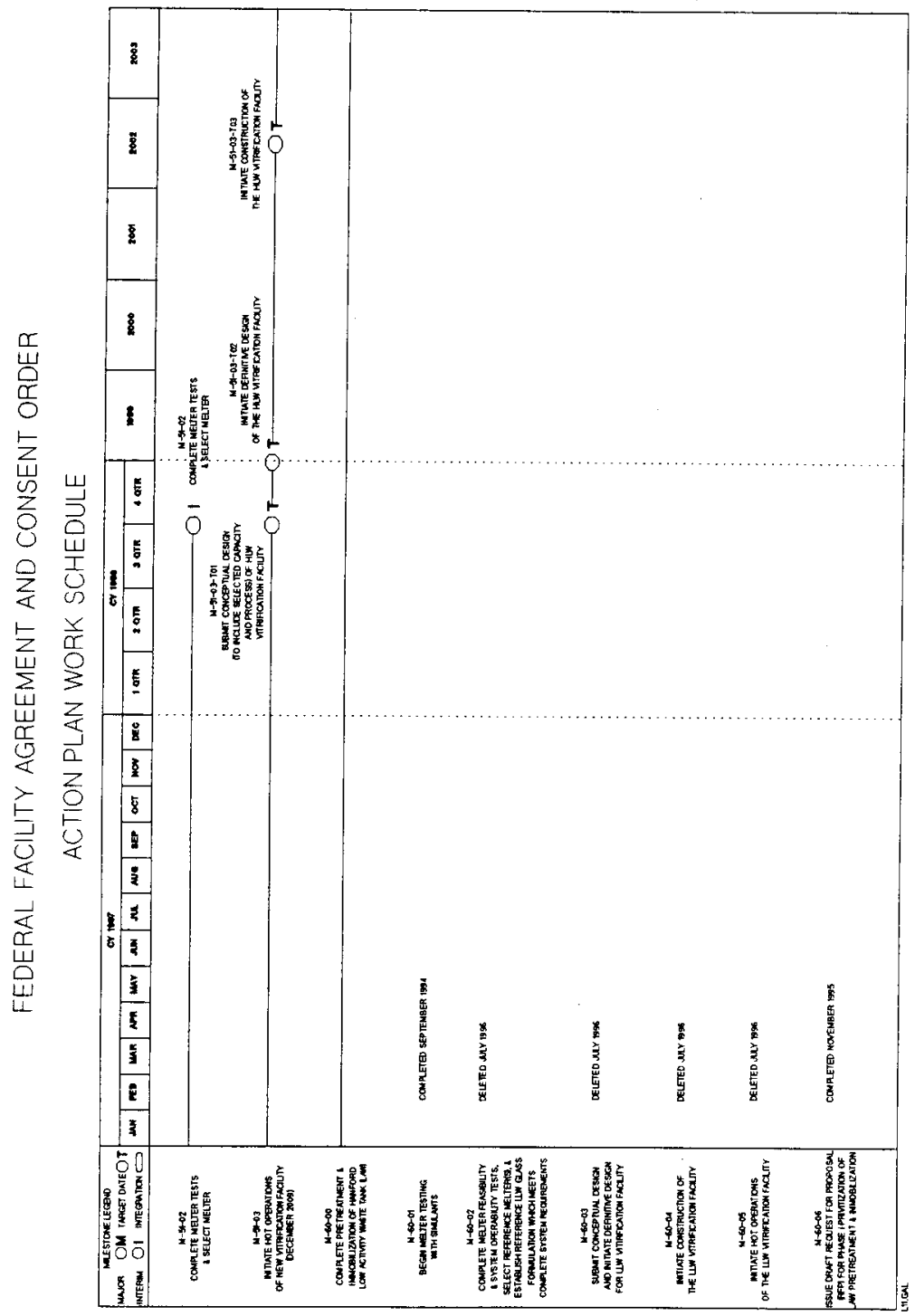

F2-1.8 
DOE/RL-97-14

Figure 2-1. Operating Schedules for Units Managing

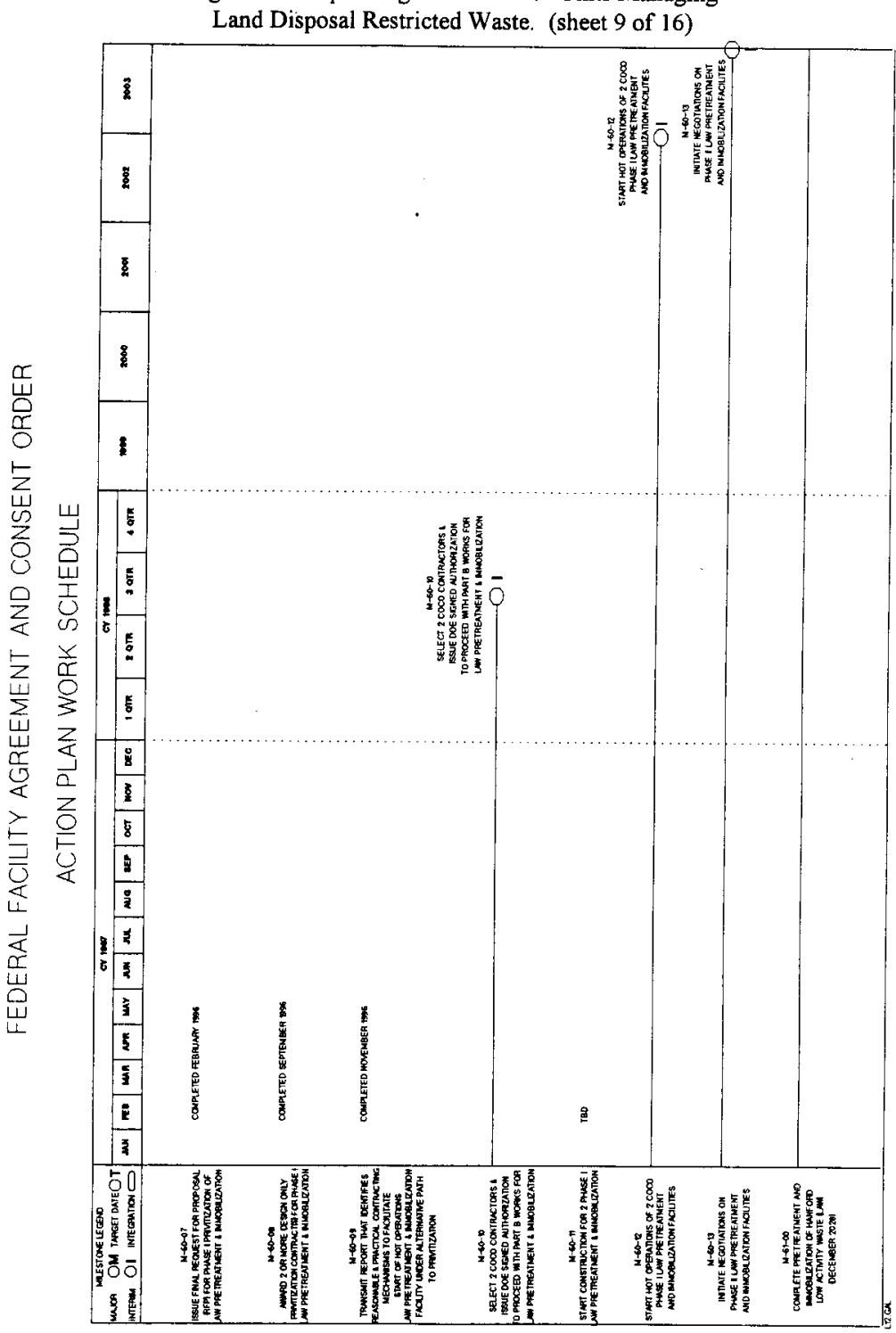

F2-1.9 
Figure 2-1. Operating Schedules for Units Managing

Land Disposal Restricted Waste. (sheet 10 of 16)

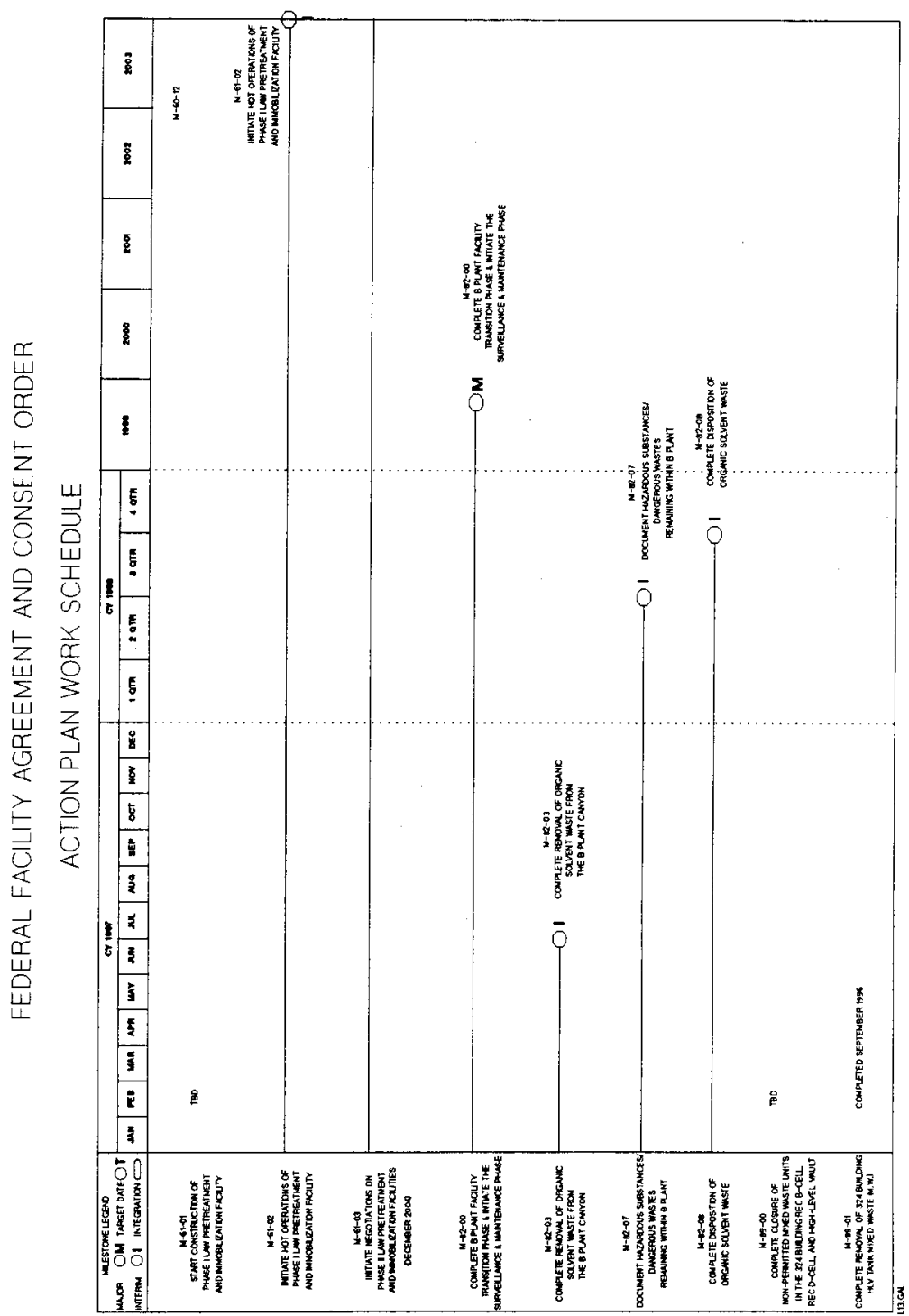

F2-1.10 
Figure 2-1. Operating Schedules for Units Managing Land Disposal Restricted Waste. (sheet 11 of 16)

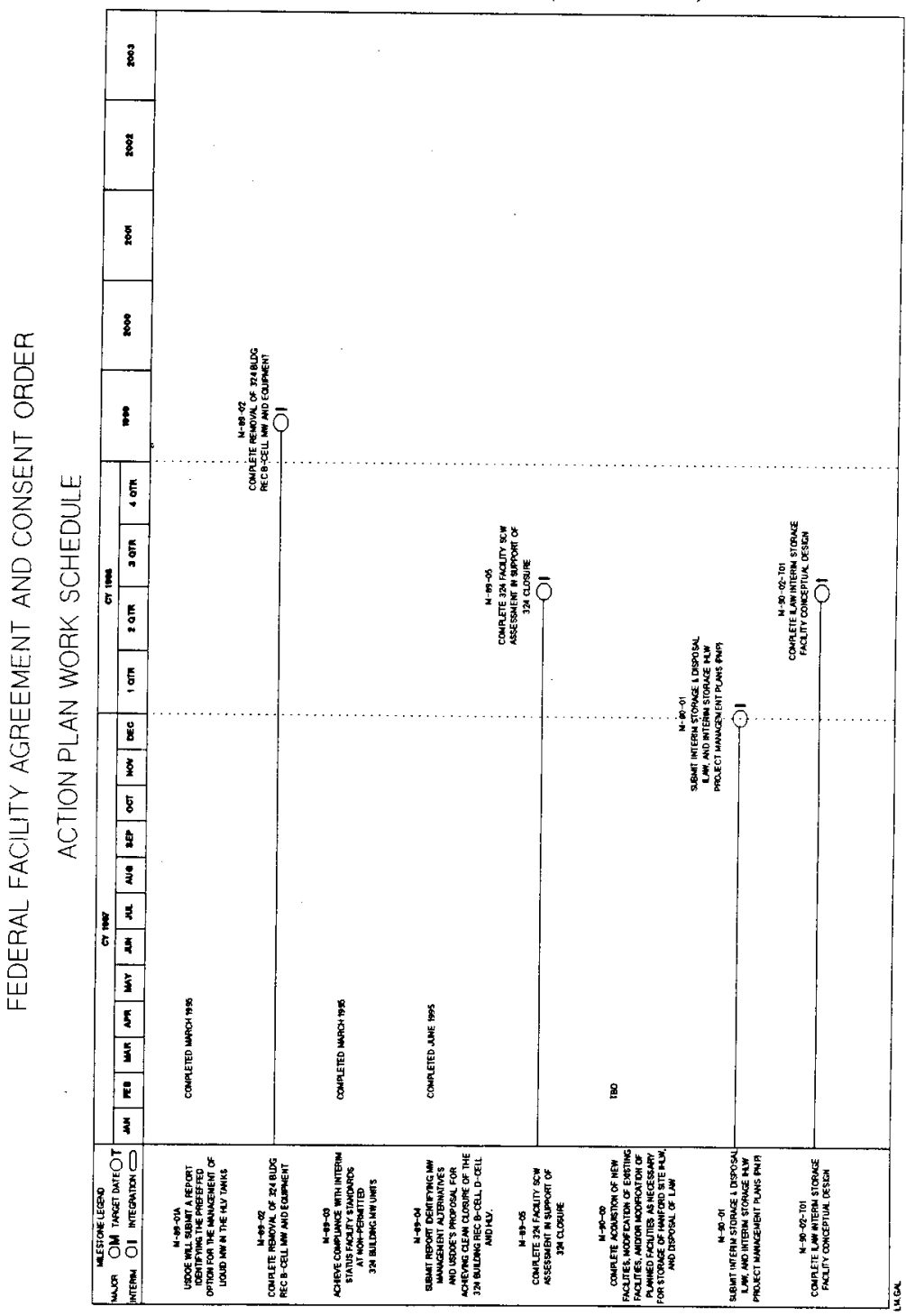

F2-1.11 
Figure 2-1. Operating Schedules for Units Managing Land Disposal Restricted Waste. (sheet 12 of 16)

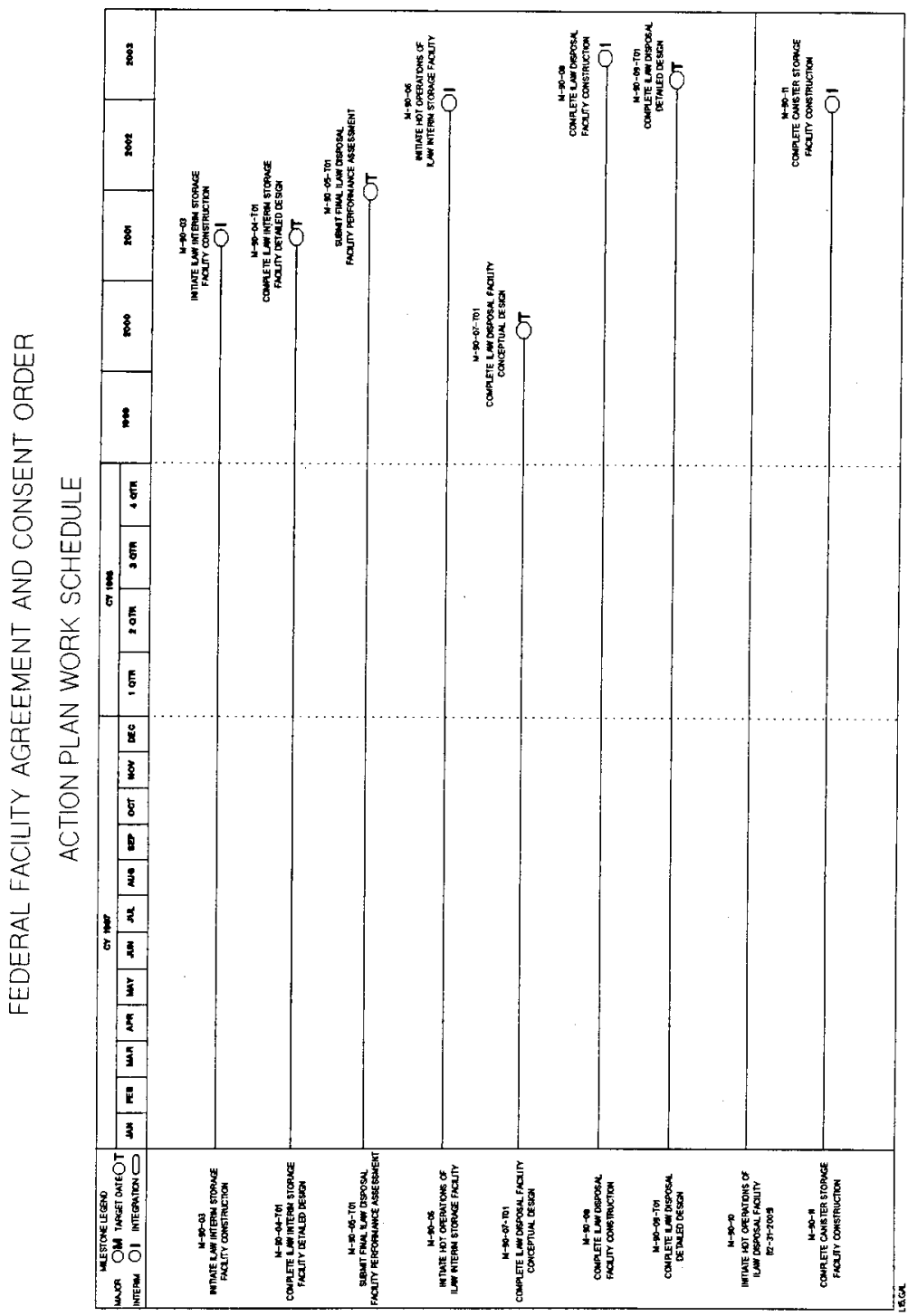

F2-1.12 
FEDERAL FACILITY AGREEMENT AND CONSENT ORDER ACTION PLAN WORK SCHEDULE

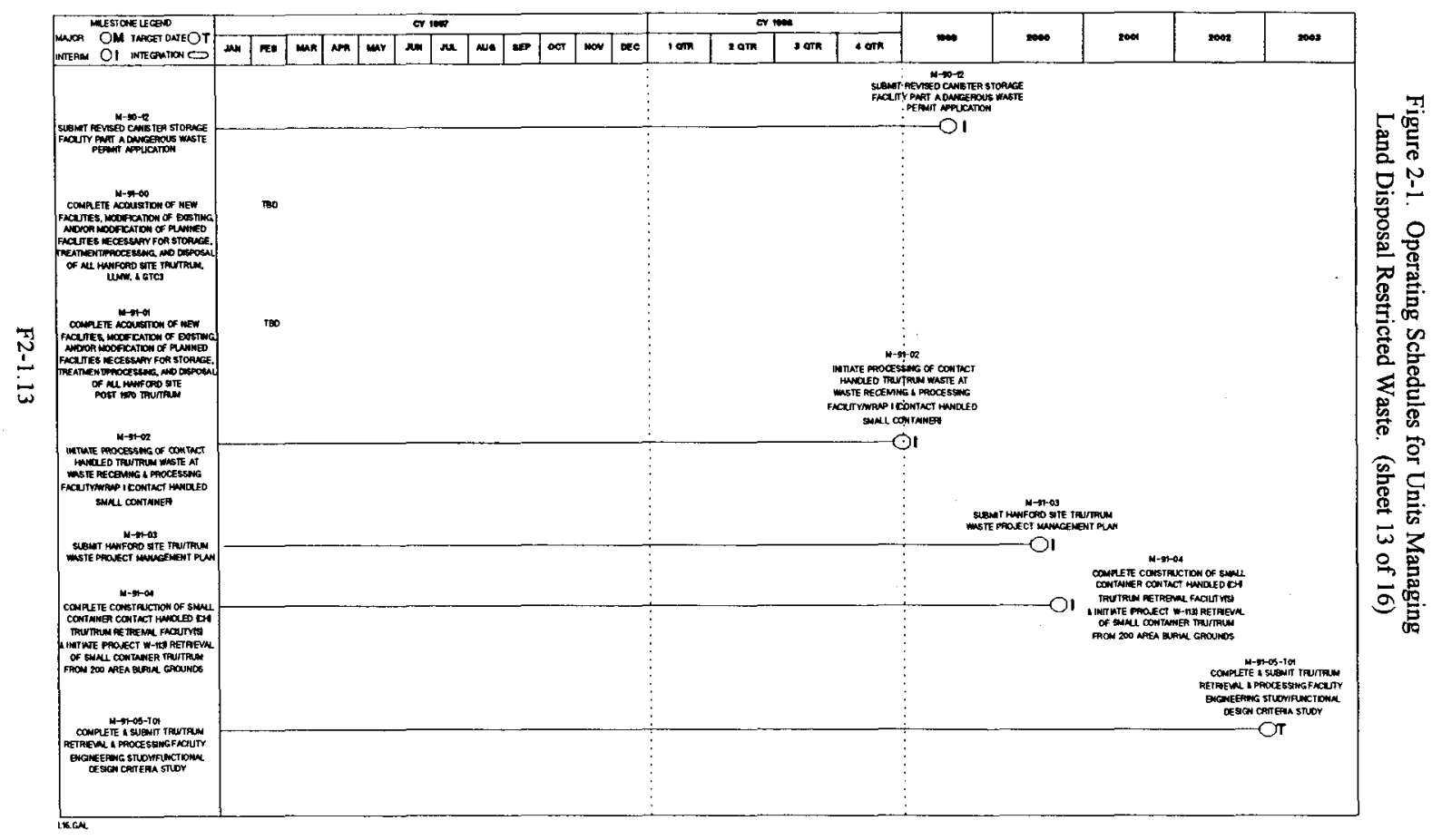


FEDERAL FACILITY AGREEMENT AND CONSENT ORDER

ACTION PLAN WORK SCHEDULE

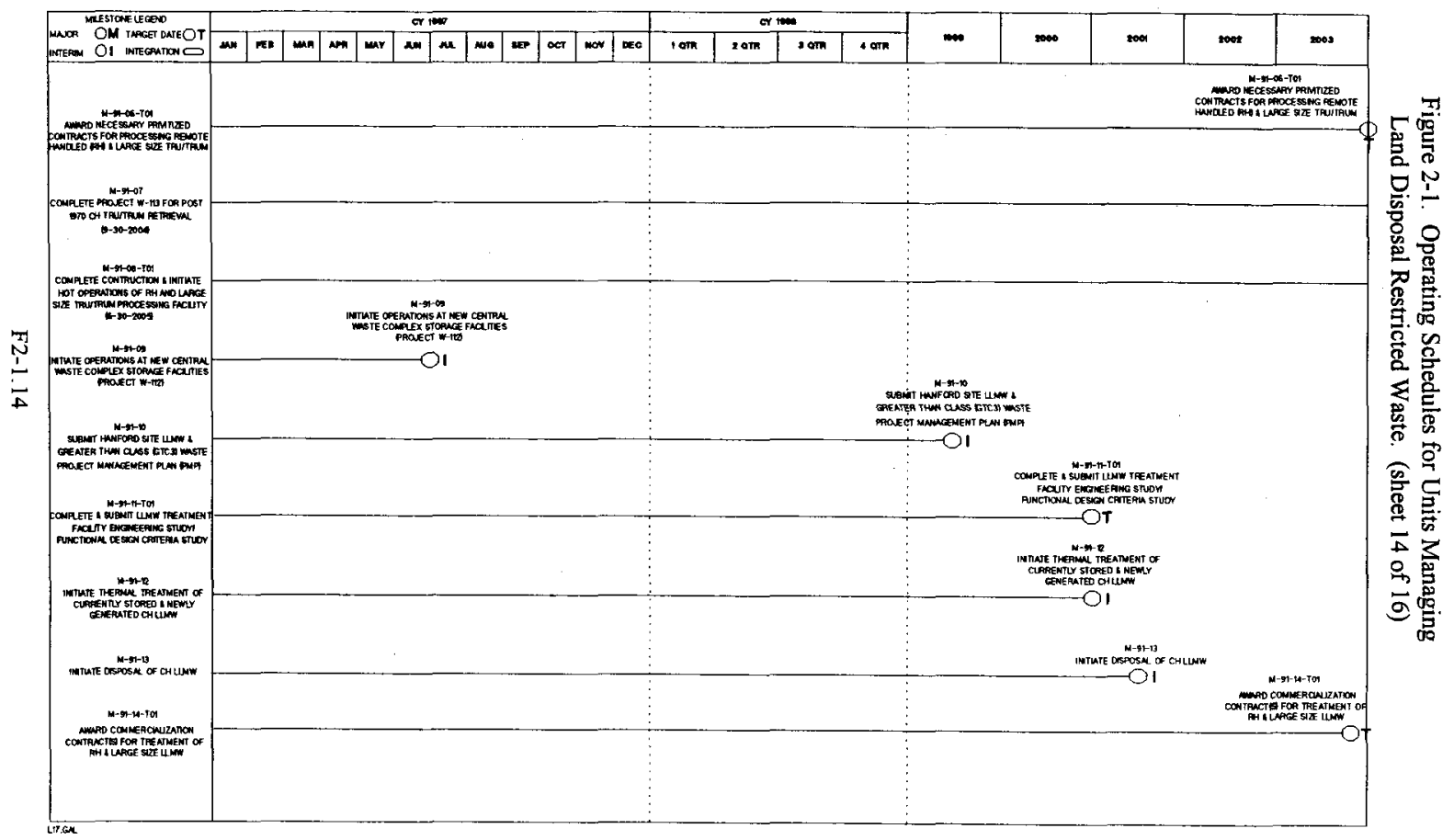


- fEDERAL FACILITY AGREEMENT AND CONSENT ORDER

ACTION PLAN WORK SCHEDULE

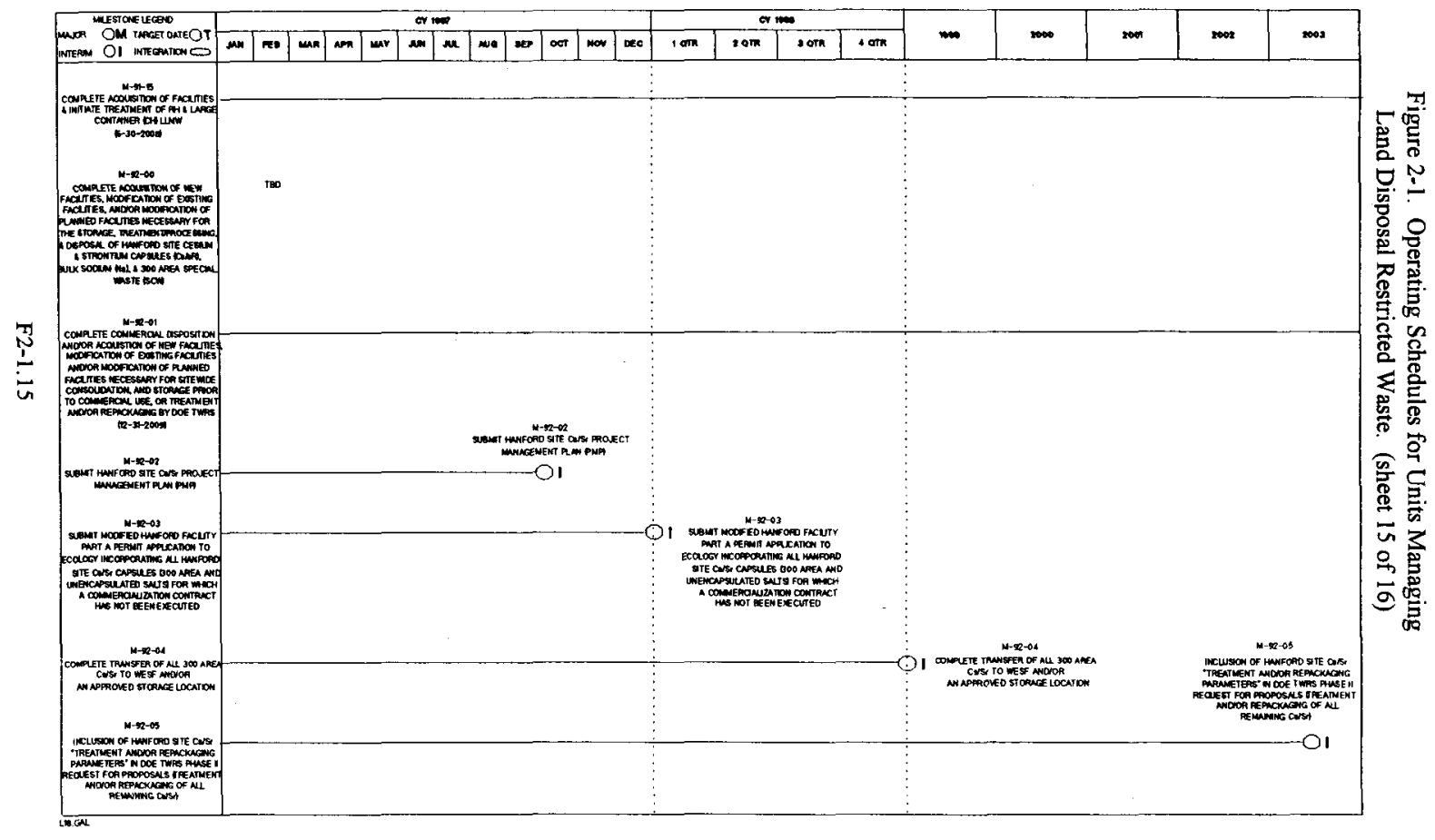


FEDERAL FACILITY AGREEMENT AND CONSENT ORDER ACTION PLAN WORK SCHEDULE

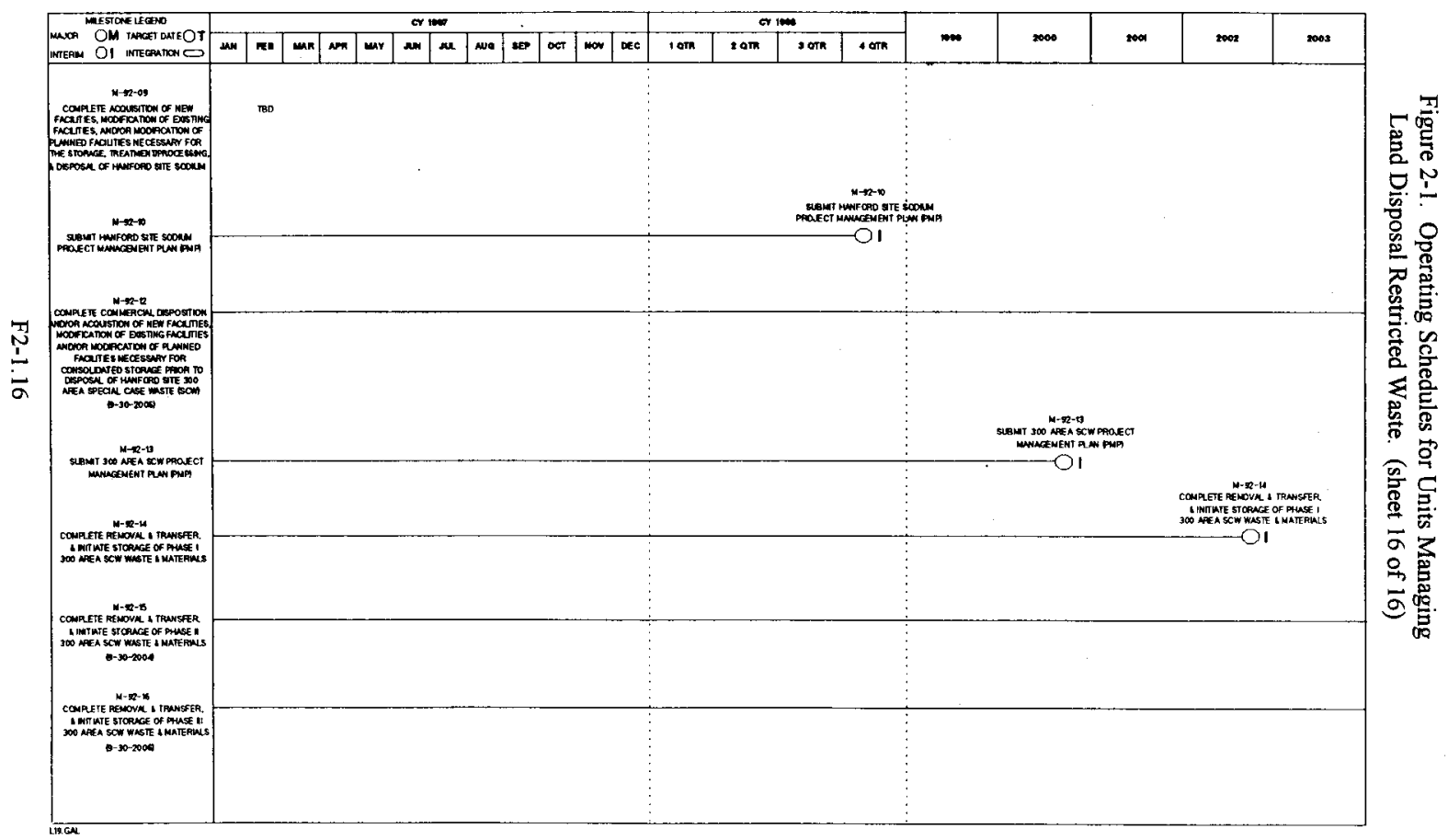


Figure 2-2. Hanford Tank Waste Remediation System.

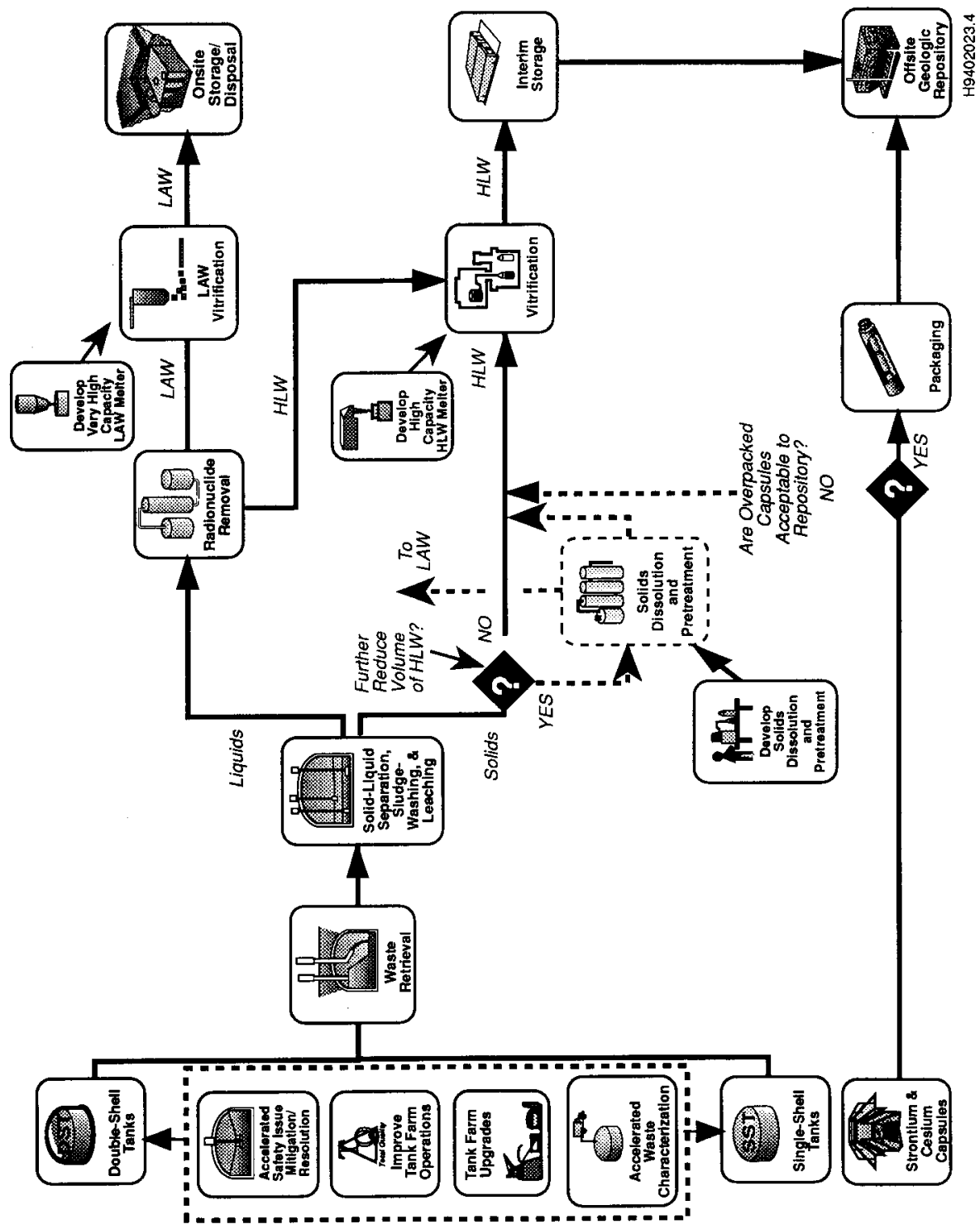


Figure 2-3. Central Waste Complex Stored Waste and

Retrievably Stored Waste Treatment Flow Diagram.

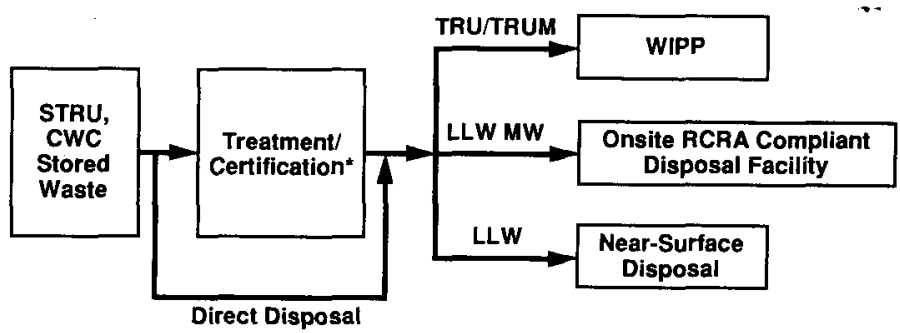

Legend
CWC = Central Waste Complex
LLW $=$ Low-Level Waste
$M W=$ Mixed Waste
RCRA = Resource Conservation and Recovery Act
STRU = Suspect TRU and TRU Waste

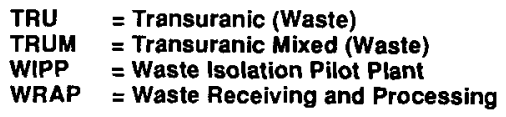

* Onsite treatment and/or offsite commercial treatment. 
Table 2-1. Summary of Annual Waste Generation or Receipt Projections."

\begin{tabular}{|c|c|c|c|c|c|}
\hline \multirow{2}{*}{ Waste stream } & \multicolumn{5}{|c|}{ Projected generation or receipt $\left(\mathrm{m}^{3}\right)$} \\
\hline & 1997 & 1998 & 1999 & 2000 & 2001 \\
\hline 1. DST Waste (before evaporation) & 16,500 & 15,700 & 7,800 & 8,100 & 4,000 \\
\hline 2. SST Waste & 0.00 & 0.00 & 0.00 & 0.00 & 0.00 \\
\hline 3. 242-A Evaporator Process Condensate & 12,100 & 16,100 & 11,000 & 4,100 & 3,700 \\
\hline 4. PUREX Storage Tunnels Waste ${ }^{b}$ & 0.00 & 0.00 & 0.00 & 0.00 & 0.00 \\
\hline 5. PUREX Containment Building ${ }^{b}$ & 0.00 & 0.00 & 0.00 & 0.00 & 0.00 \\
\hline 6. CWC Stored Low-Level, TRU, and PCB Waste & 1,220 & 1,220 & 1,340 & 1,630 & 2,020 \\
\hline 7. Suspect-TRU and TRU Waste & 0.00 & 0.00 & 0.00 & 0.00 & 0.00 \\
\hline 8. 224-T TRUSAF Stored Waste* & 0.00 & 0.00 & 0.00 & 0.00 & 0.00 \\
\hline 9. 324 REC $^{d}$ & 1.3 & 1.3 & 1.3 & 0.00 & 0.00 \\
\hline 10. $324 \mathrm{HLV}$ & 0.00 & 0.00 & 0.00 & 0.00 & 0.00 \\
\hline 11. B Plant Cell 4 Waste ${ }^{f}$ & 0.2 & 0.00 & 0.00 & 0.00 & 0.00 \\
\hline 12. B Plant Containment Building Storage ${ }^{8}$ & 0.00 & 0.00 & 0.00 & 0.00 & 0.00 \\
\hline 13. B Plant Organic Waste & 0.00 & 0.00 & 0.00 & 0.00 & 0.00 \\
\hline Total Projected Generation & 29,821 & 33,021 & 20,141 & 13,830 & 9,720 \\
\hline
\end{tabular}

These rates are based on the assumptions of Chapter 1.0, Section 1.2. Depending on the stream, figures are for either generation or receipt of waste.

'Generation rate depends upon the need to move failed equipment or waste materials containing mercury, barium, mineral oil, chromium, lead, cadmium, and/or silver into the PUREX tunnels or containment building. At this point, there are no plans to add waste. However, waste generated during 324 Building stabilization may be stored in the tunnels.

"Submarine reactor comparments not included in estimates.

These generation estimates are based on the assumption that used HEPA filters in the cells may contain hazardous waste.

"224-T is currently used for temporary storage. The building is expected to be emptied in FY 1997 and closed in FY 1998.

These quantities reflect only expected mixed waste generation. Additional containers of low-level waste may also be stored in Cell 4.

Actual generation will depend on deactivation and decisions on what to leave in the canyon. Data are not available to estimate this quantity.

CWC = Central Waste Complex.

DST = Double-shell tank.

HEPA = high-efficiency particulate air.

HLV = High-Level Vault.

PCB = Polychlorinated biphenyl.
PUREX = Plutonium-Uranium Extraction (Plant).

REC = Radiochemical Engineering Cells.

SST $\quad=$ Single-shell tank.

IRU = Transuranic.

TRUSAF = Transuranic Waste Storage and Assay Facility. 
Table 2-2. Waste Stream Characterization. (sheet 1 of 4)

\begin{tabular}{|c|c|c|c|}
\hline & Waste stream & Schedule & $\begin{array}{l}\text { Method, protocol, } \\
\text { specific analyses }\end{array}$ \\
\hline 1 & 1. DST Waste & $\begin{array}{l}\text { 1994-1999 (M-44-00, } \\
\text { Ecology et al. 1989) }\end{array}$ & $\begin{array}{l}\text { A Tank Waste Analysis Plan was developed using the results of } \\
\text { the data quality objective process for characterization of all tanks. } \\
\text { A DST-specific plan is also being prepared. } \\
\text { Specific analysis will be determined by the data quality objectives } \\
\text { process. } \\
\text { A tank characterization plan for each applicable tank will also be } \\
\text { developed using inputs from the data quality objectives process. } \\
\text { The tank characterization plans will integrate the results of the } \\
\text { various issue and process efforts into a specific sampling and } \\
\text { analysis plan for a given tank. }\end{array}$ \\
\hline & SST Waste & 1994-1999 (M-44-00) & $\begin{array}{l}\text { The number of core samples from each SST will be determined } \\
\text { by the data quality objectives process. } \\
\text { Samples will be analyzed according to the individual tank } \\
\text { characterization plan. } \\
\text { Data will be reported in a tank characterization report. }\end{array}$ \\
\hline 1 & $\begin{array}{l}\text { 3. 242-A Evaporator } \\
\text { Process Condensate }\end{array}$ & $\begin{array}{l}\text { Waste to be sampled in } \\
\text { accordance with LERF/ETF } \\
\text { waste analysis plan }\end{array}$ & $\begin{array}{l}\text { A waste analysis plan was developed for sampling process } \\
\text { condensate in the LERF basins for processing at the } 200 \text { Area } \\
\text { ETF. }\end{array}$ \\
\hline 1 & $\begin{array}{l}\text { PUREX Storage } \\
\text { Tunnels Waste }\end{array}$ & $\begin{array}{l}\text { Waste characterization } \\
\text { complete }\end{array}$ & - $\quad$ Characterization details contained in RL (1990b). \\
\hline l & $\begin{array}{l}\text { 5. PUREX Containment } \\
\text { Building }\end{array}$ & $\begin{array}{l}\text { No further characterization is } \\
\text { planned }\end{array}$ & NA \\
\hline
\end{tabular}


Table 2-2. Waste Stream Characterization. (sheet 2 of 4)

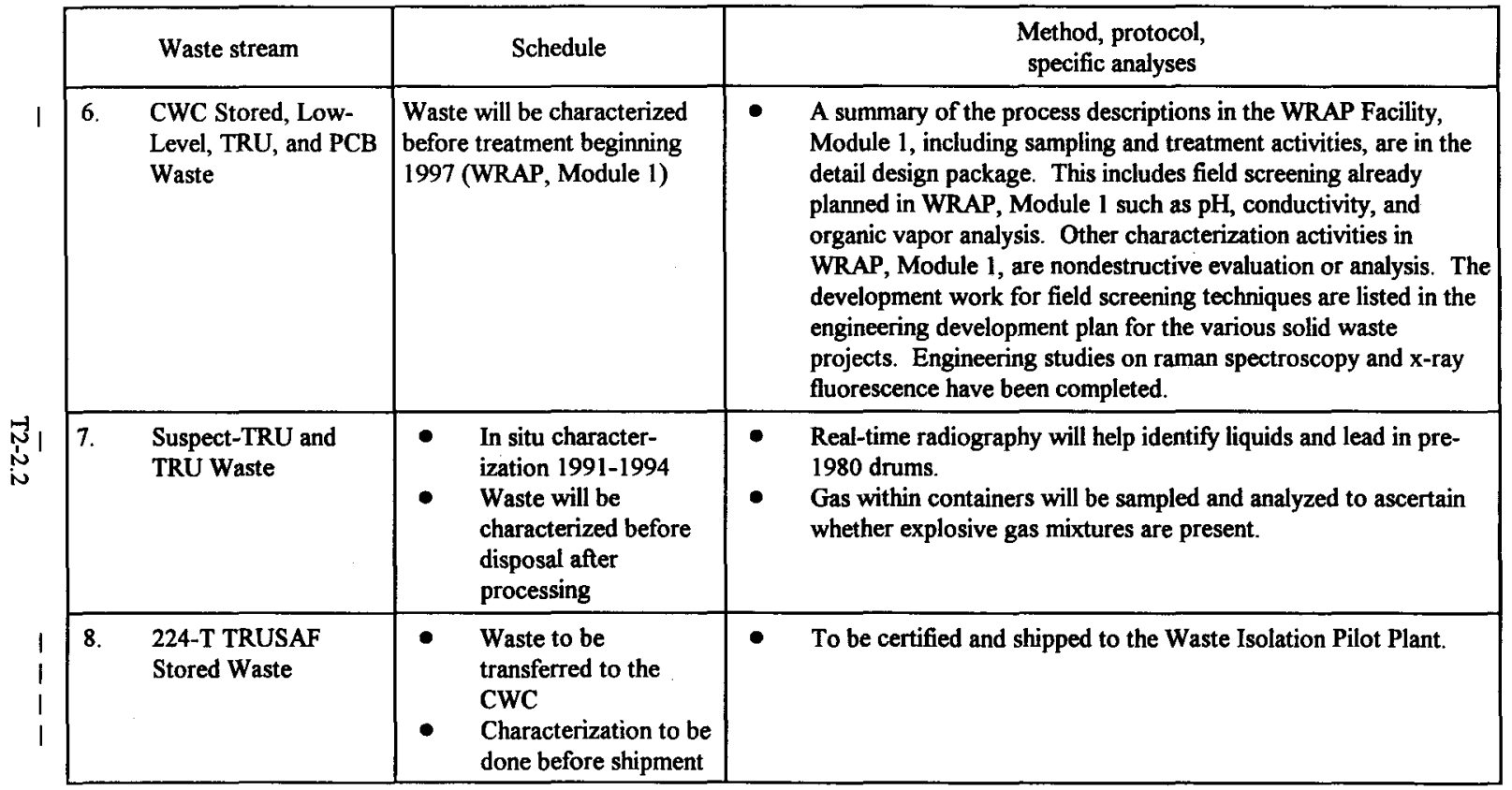


Table 2-2. Waste Stream Characterization. (sheet 3 of 4)

\begin{tabular}{|c|c|c|}
\hline Waste stream & Schedule & $\begin{array}{l}\text { Method, protocol, } \\
\text { specific analyses }\end{array}$ \\
\hline 324 REC & Complete & $\begin{array}{l}\text { All known waste streams characterized based on analysis or process } \\
\text { knowledge. }\end{array}$ \\
\hline $324 \mathrm{HLV}$ & Complete & $\begin{array}{l}\text { All waste was designated based on analysis of waste streams and process } \\
\text { knowledge. (Waste is no longer at } 324 \mathrm{HLV} \text {.) }\end{array}$ \\
\hline B Plant Cell 4 Waste & $\begin{array}{l}\text { No further characterization is } \\
\text { planned for this waste }\end{array}$ & $\begin{array}{l}\text { Process knowledge provides sufficient information to characterize this } \\
\text { waste. }\end{array}$ \\
\hline $\begin{array}{l}\text { B Plant Containment } \\
\text { Building Storage }\end{array}$ & $\begin{array}{l}\text { No further characterization is } \\
\text { planned for this waste }\end{array}$ & Process knowledge will be used to characterize for deactivation. \\
\hline
\end{tabular}


Table 2-2. Waste Stream Characterization. (sheet 4 of 4)

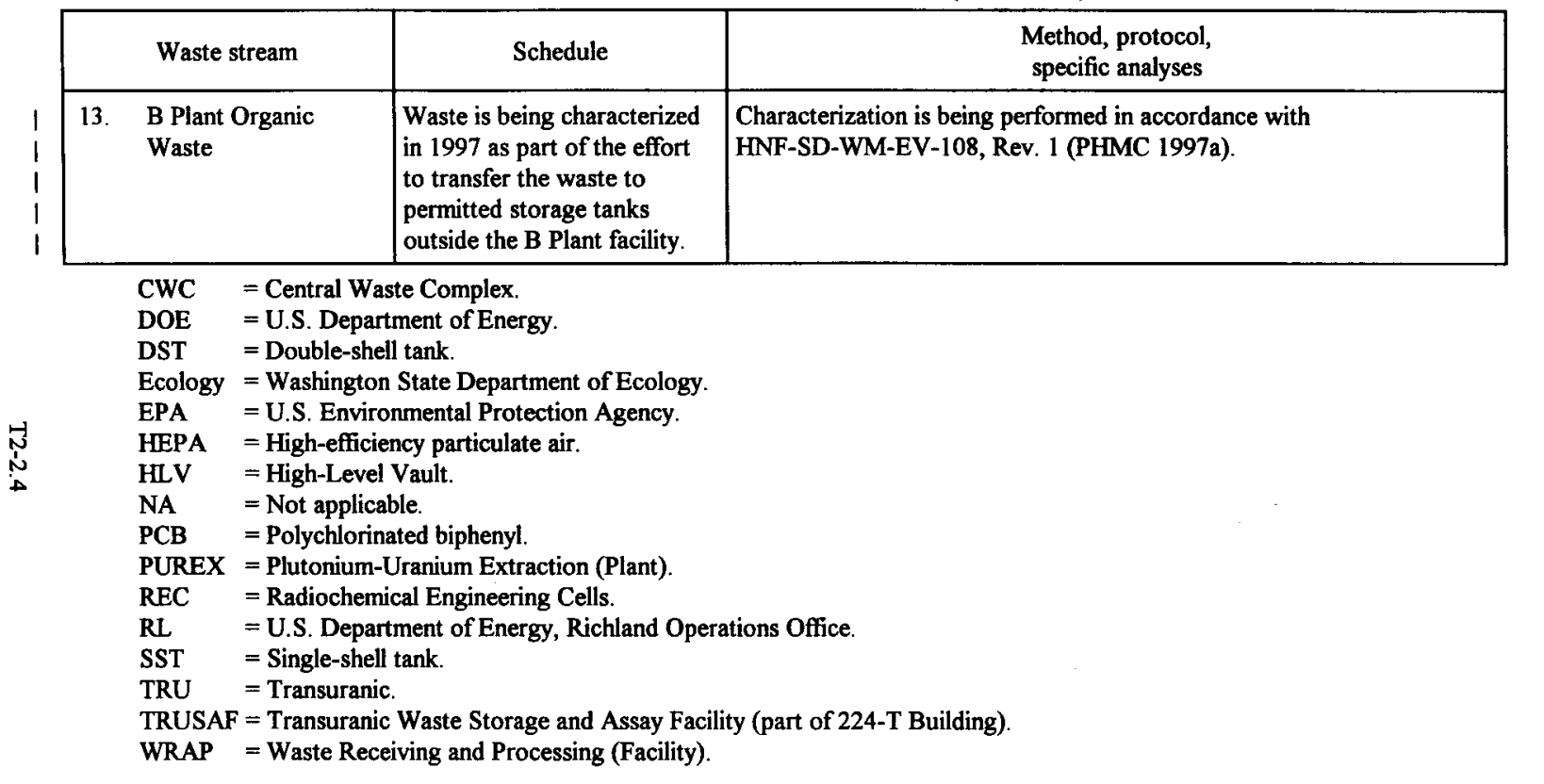


Table 2-3. Dangerous Waste Designations." (sheet 1 of 6 )

\begin{tabular}{|c|c|}
\hline Waste stream & Designated waste code(s) \\
\hline 1. DST Waste W,d $^{\mathrm{b}}$ & $\begin{array}{l}\text { D001 (ignitable) }{ }^{\mathrm{a}, \mathrm{c}} \\
\text { D002 (corrosive) } \\
\text { D003 (reactive) } \\
\text { D004 (TCLP arsenic) } \\
\text { D005 (TCLP barium) } \\
\text { D006 (TCLP cadmium) } \\
\text { D007 (TCLP chromium) } \\
\text { D008 (TCLP lead) } \\
\text { D009 (TCLP mercury) } \\
\text { D010 (TCLP selenium) } \\
\text { D01 } \text { (TCLP silver) }_{\text {F001 (1,1,1-trichloroethane) }} \\
\text { F002 (methylene chloride) } \\
\text { F003 (acetone and hexone) } \\
\text { F004 (o-cresol, p-cresol) } \\
\text { F005 (methyl ethyl ketone) } \\
\text { F039 (multisource leachate) } \\
\text { WP01 (persistent extremely hazardous } \\
\text { waste) } \\
\text { WP02 (persistent dangerous waste) } \\
\text { WT01 (toxic, extremely hazardous waste) } \\
\text { WT02 (toxic, dangerous waste) }\end{array}$ \\
\hline
\end{tabular}


Table 2-3. Dangerous Waste Designations." (sheet 2 of 6 )

\begin{tabular}{|c|c|c|}
\hline \multicolumn{2}{|r|}{ Waste stream } & Designated waste code(s) \\
\hline & SST Waste ${ }^{\mathrm{b}}$ & $\begin{array}{l}\text { D001 (ignitable) } \\
\text { D002 } \\
\text { (corrosive) }^{c} \\
\text { D003 (reactive) } \\
\text { D004 (TCLP arsenic) } \\
\text { D005 (TCLP barium) } \\
\text { D006 (TCLP cadmium) } \\
\text { D007 (TCLP chromium) } \\
\text { D008 (TCLP lead) } \\
\text { D009 (TCLP mercury) } \\
\text { D010 (TCLP selenium) } \\
\text { D011 (TCLP silver) } \\
\text { F001 (1,1,1-trichloroethane) } \\
\text { F002 (methylene chloride) } \\
\text { F003 (acetone and hexone) } \\
\text { F004 (o-cresol, p-cresol) } \\
\text { F005 (methyl ethyl ketone) } \\
\text { WT01 (toxic, extremely hazardous waste) } \\
\text { WT02 (toxic, dangerous waste) } \\
\text { WP01 (persistent extremely hazardous waste) } \\
\text { WP02 (persistent dangerous waste) }\end{array}$ \\
\hline & $\begin{array}{l}\text { 242-A Evaporator Process } \\
\text { condensate }^{d}\end{array}$ & $\begin{array}{l}\text { F001 (1,1,1-trichloroethane) } \\
\text { F002 (methylene chloride) } \\
\text { F003 (acetone, hexone) } \\
\text { F004 (o-cresol, p-cresol) } \\
\text { F005 (methyl ethyl ketone) } \\
\text { WT02 (toxic, dangerous waste) }\end{array}$ \\
\hline & PUREX Storage Tunnels Waste & $\begin{array}{l}\text { D001 (ignitable) } \\
\text { D005 (TCLP barium) } \\
\text { D006 (TCLP cadmium) } \\
\text { D007 (TCLP chromium) } \\
\text { D008 (TCLP lead) } \\
\text { D009 (TCLP mercury) } \\
\text { D011 (TCLP silver) } \\
\text { WT02 (toxic, dangerous waste) (mineral oil) }\end{array}$ \\
\hline & PUREX Containment Building & $\begin{array}{l}\text { D007 (TCLP chromium) } \\
\text { WT01 (toxic, extremely hazardous waste) }\end{array}$ \\
\hline
\end{tabular}


Table 2-3. Dangerous Waste Designations, (sheet 3 of 6)

\begin{tabular}{|c|c|c|}
\hline & Waste stream & Designated waste code(s) \\
\hline 6. & $\begin{array}{l}\text { CWC Stored Low-Level, TRU, and } \\
\text { PCB Waste } \\
\text { NOTE: Because of the nature of this } \\
\text { TSD unit, an extensive number of } \\
\text { waste codes apply. Some of the } \\
\text { major codes are presented here. } \\
\text { (This also applies to Table } 2-6 \text {.) The } \\
\text { Part A Form } 3 \text { permit application } \\
\text { contains a complete listing. Not all } \\
\text { codes are being managed that appear } \\
\text { on the Part A Form } 3 \text { (RL 1989a). }\end{array}$ & $\begin{array}{l}\text { D001 (ignitable) } \\
\text { D002 (corrosive) } \\
\text { D003 (reactive) } \\
\text { D004 (TCLP arsenic) } \\
\text { D005 (TCLP barium) } \\
\text { D006 (TCLP cadmium) } \\
\text { D007 (TCLP chromium) } \\
\text { D008 (TCLP lead) } \\
\text { D009 (TCLP mercury) } \\
\text { D010 (TCLP selenium) } \\
\text { D011 (TCLP silver) } \\
\text { D012 (TCLP Endrin) } \\
\text { D016 (TCLP 2,4-D) } \\
\text { D039 (perchlorethylene) } \\
\text { F001 (spent halogenated degreasing solvents) } \\
\text { F002 (spent halogenated solvents) } \\
\text { F003 (spent solvents) } \\
\text { F004 (spent solvents) } \\
\text { F005 (spent non-halogenated solvents) } \\
\text { F039 (multisource leachate) } \\
\text { P029 (copper cyanides) } \\
\text { P030 (soluble cyanide salts) } \\
\text { P098 (potassium cyanide) } \\
\text { P106 (sodium cyanide) } \\
\text { P120 (vanadium pentoxide) } \\
\text { U080 (dichloromethane) } \\
\text { U123 (formic acid) } \\
\text { U161 (methylisobutylketone) } \\
\text { W001 (PCBs) } \\
\text { WP01 (persistent extremely hazardous waste) } \\
\text { WP02 (persistent dangerous waste) } \\
\text { WT01 (toxic, extremely hazardous waste) } \\
\text { WT02 (toxic, dangerous waste) } \\
\text { Dong }\end{array}$ \\
\hline
\end{tabular}


Table 2-3. Dangerous Waste Designations." (sheet 4 of 6)

\begin{tabular}{|l|l|}
\hline \multicolumn{1}{|c|}{ Waste stream } & \multicolumn{1}{|c|}{ Designated waste code(s) } \\
\hline 7. Suspect-TRU and TRU Waste & D001 (ignitable) \\
& D003 (reactive) \\
& D005 (TCLP barium) \\
& D006 (TCLP cadmium) \\
& D007 (TCLP chromium) \\
& D008 (TCLP lead) \\
& D009 (TCLP mercury) \\
& D011 (TCLP silver) \\
& F001 (spent halogenated degreasing solvents) \\
& F003 (spent solvents) \\
& F005 (spent non-halogenated solvents) \\
& P015 (beryllium dust) \\
& WP01 (persistent extremely hazardous waste) \\
& WT01 (toxic, extremely hazardous waste) \\
& WT02 (toxic, dangerous waste) \\
\hline 8. & D002 (corrosive) \\
& D005 (TCLP barium) \\
& D006 (TCLP cadmium) \\
& D007 (TCLP chromium) \\
& D008 (TCLP lead) \\
& D009 (TCLP mercury) \\
& WP01 (persistent extremely hazardous waste) \\
& WT01 (toxic, extremely hazardous waste) \\
\hline
\end{tabular}


Table 2-3. Dangerous Waste Designations." (sheet 5 of 6 )

\begin{tabular}{|c|c|c|}
\hline \multicolumn{2}{|r|}{ Waste stream } & Designated waste code(s) \\
\hline & 324 REC & $\begin{array}{l}\text { D005 (TCLP barium) } \\
\text { D006 (TCLP cadmium) } \\
\text { D007 (TCLP chromium) } \\
\text { D008 (TCLP lead) } \\
\text { WT01 (toxic, extremely hazardous waste) } \\
\text { WT02 (toxic, dangerous waste) }\end{array}$ \\
\hline 10 & $\begin{array}{l}324 \mathrm{HLV} \\
\text { Note: This waste has been treated and } \\
\text { residues moved to the } 324 \text { REC }\end{array}$ & \\
\hline 11. & B Plant Cell 4 Waste & D008 (TCLP lead) \\
\hline & $\begin{array}{l}\text { B Plant Containment Building } \\
\text { Storage }\end{array}$ & $\begin{array}{l}\text { D004 } \text { (TCLP arsenic) }^{\mathrm{e}} \\
\text { D005 } \text { (TCLP barium) }^{\mathrm{e}} \\
\text { D006 (TCLP cadmium) } \\
\text { D007 } \text { (TCLP chromium) }^{\mathrm{e}} \\
\text { D008 (TCLP lead) } \\
\text { D009 } \text { (TCLP mercury) }^{\mathrm{e}} \\
\text { D010 } \text { (TCLP selenium) }^{\mathrm{e}} \\
\text { D011 (TCLP silver) } \\
\text { F001 (1,1,1-trichloroethane) } \\
\text { F002 (methylene chloride) } \\
\text { F003 (acetone, hexone) }^{\mathrm{f}} \\
\text { F004 (o-cresol, p-cresol) } \\
\text { F005 (methyl ethyl ketone) } \\
\text { WT01 (toxic, extremely hazardous waste) } \\
\text { WT02 (toxic, dangerous waste) }\end{array}$ \\
\hline
\end{tabular}


Table 2-3. Dangerous Waste Designations." (sheet 6 of 6 )

\begin{tabular}{|l|l|}
\hline \multicolumn{1}{|c|}{ Waste stream } & \multicolumn{1}{|c|}{ Designated waste code(s) } \\
\hline 13. B Plant Organic Waste & D004 (TCLP arsenic) \\
& D005 (TCLP barium) \\
& D006 (TCLP cadmium) \\
& D007 (TCLP chromium) \\
& D008 (TCLP lead) \\
& D009 (TCLP mercury) \\
& D010 (TCLP selenium) \\
& D011 (TCLP silver) \\
& F001 (1,1,1-trichloroethane) \\
& F002 (methylene chloride) \\
& F003 (acetone, hexone) \\
& F004 (o-cresol, p-cresol) \\
& F005 (methyl ethyl ketone) \\
& WT02 (toxic, dangerous waste) \\
\hline
\end{tabular}

${ }^{2}$ Further information is given in Section 2.2 .

${ }^{6}$ TCLP waste codes D018, D019, D022, D028, D029, D030, D033, D034, D035, D036, and D038 through D043 are listed in the Part A, Form 3 Permit application, but are not listed in this table or in Table 2-6. Analysis of tank waste has not yet confirmed these to be present, although analysis alone cannot determine LDR status.

'Designation is based on process knowledge and dilution prohibition. Waste in storage does not display characteristic.

I 'The F039 waste code has been added to these TSD units' Part A Form 3 permit applications, but no F039 waste is currently being managed.

'These waste codes are included on the B Plant Complex Part A Permit Application, Form 3, Revision 5, but no analytical data are present to indicate that these constituents actually are present. They are included because they potentially may be present.

fThese waste codes are included to be consistent with the mixture and derived-from rules, because the process equipment at B Plant processed tank waste.

CWC = Central Waste Complex.

DST = Double-shell tank.

HLV = High-Level Vault.

PCB = Polychlorinated biphenyl.

PUREX = Plutonium-Uranium Extraction (Plant).

REC $=$ Radiochemical Engineering Cell.

SST = Single-shell tank.

TCLP = Toxic characteristic leaching procedure.

TRU $=$ Transuranic

TRUSAF $=224-\mathrm{T}$ Transuranic Waste Storage and Assay Facility. 
Table 2-4. Storage Unit Characteristics. (sheet 1 of 3)

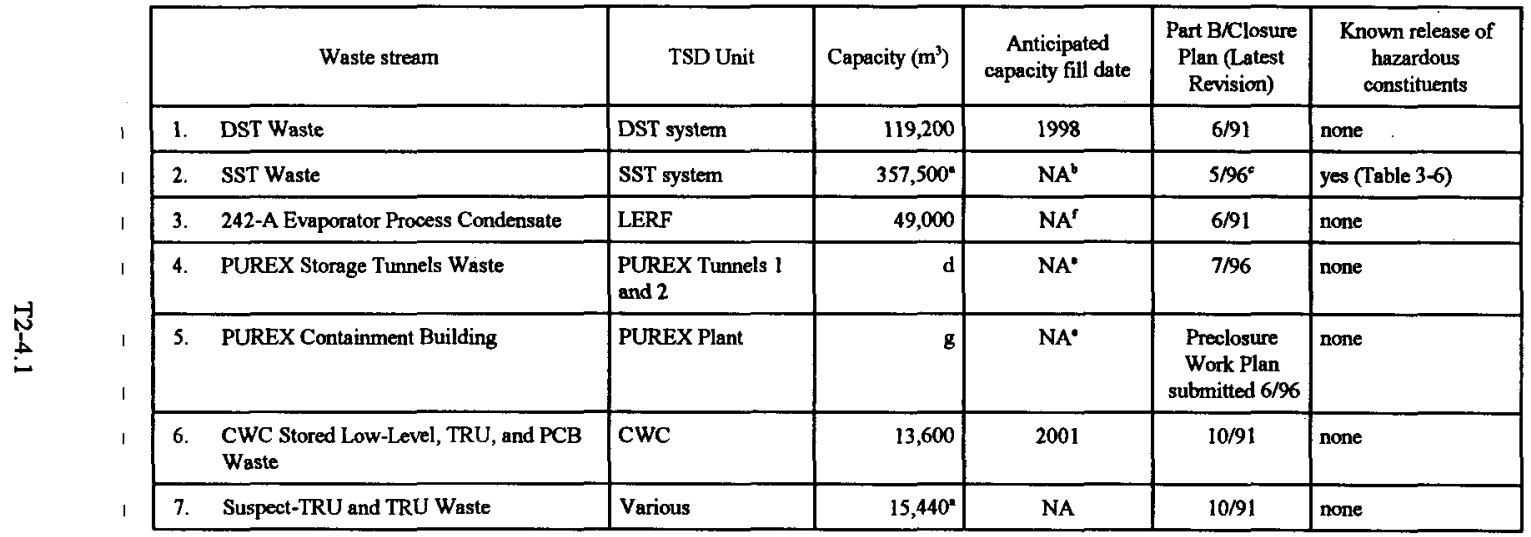


Table 2-4. Storage Unit Characteristics. (sheet 2 of 3)

\begin{tabular}{|c|c|c|c|c|c|c|}
\hline \multirow{3}{*}{ I } & Waste stream & TSD Unit & Capacity $\left(\mathrm{m}^{3}\right)$ & $\begin{array}{l}\text { Anticipated } \\
\text { capacity fill date }\end{array}$ & $\begin{array}{l}\text { Part B/Closure } \\
\text { Plan (Latest } \\
\text { Revision) }\end{array}$ & $\begin{array}{c}\text { Known release of } \\
\text { hazardous } \\
\text { constituents }\end{array}$ \\
\hline & 8. 224-T TRUSAF Stored Waste & 224-T TRUSAF & 420 & NA & $6 / 92^{\mathrm{j}}$ & none \\
\hline & 9. $324 \mathrm{REC}$ & 324 Building ${ }^{h}$ & 46.6 & NA & $12 / 95^{\circ}$ & none \\
\hline 1 & 10. $324 \mathrm{HLV}$ & 324 Building & $56.7^{\circ}$ & NA & $12 / 95^{\circ}$ & none \\
\hline & 11. B Plant Cell 4 Waste & B Plant Complex & 51 & NA & $\begin{array}{c}\text { Preclosure } \\
\text { Work Plan } \\
\text { due 3/99 }\end{array}$ & none \\
\hline & 12. B Plant Containment Building Storage & B Plant Complex & $\mathrm{i}$ & NA & $\begin{array}{l}\text { Preclosure } \\
\text { Work Plan } \\
\text { due 3/99 }\end{array}$ & none \\
\hline
\end{tabular}


Table 2-4. Storage Unit Characteristics. (sheet 3 of 3)

\begin{tabular}{|c|c|c|c|c|c|}
\hline Waste stream & TSD Unit & Capacity $\left(\mathrm{m}^{3}\right)$ & $\begin{array}{c}\text { Anticipated } \\
\text { capacity fill date }\end{array}$ & $\begin{array}{c}\text { Part B/Closure } \\
\text { Plan (Latest } \\
\text { Revision) }\end{array}$ & $\begin{array}{c}\text { Known release of } \\
\text { bazardous } \\
\text { constituents }\end{array}$ \\
\hline 13. B Plant Organic Waste & B Plant Complex & 35 & NA & $\begin{array}{c}\text { Preclosure } \\
\text { Work Plan } \\
\text { due 3/99 }\end{array}$ & none \\
\hline
\end{tabular}

This unit is no longer used for active storage; capacity noted is for information only.

Wo future generation of this waste.

'Closure plan.

'PUREX Storage Tunnel 1 has a total capacity for 8 rail cars, equivalent to 4,129 cubic meters, and is filled with approximately 596 cubic meters of stored equipment. PUREX Storage Tunnel 2 has a total capacity for 40 rail cars, equivalent to 19,878 cubic meters, and currently contains 28 rail cars or 2,204 cubic meters of stored equipment. The total capacity of both tunnels is 24,007 cubic meters.

-Capacity is currently sufficient for future generation.

The LERF operates in conjunction with the ETF. Liquids exceeding LDR standards that are transferred to the LERF basin will be treated in the ETF within 1 year (flow-through mode).

The PUREX containment building has a current permit capacity of $434 \mathrm{~m}^{3}$. It currently contains $1.4 \times 10^{-4} \mathrm{~m}^{3}$ of chromium debris contaminated from E-Cell, but stored in F-Cell.

These portions of the 324 facility are not included in a TSD unit.

The total capacity of the $B$ Plant containment building is 35,170 cubic meters if the canyon and all process cells are completely filled.

This is a Part B application date. A closure plan is currently being prepared.

CWC

$=$ Central Waste Complex.

PUREX

DST

$=$ Double-shell tank.

REC

HLV

$=$ High-Level Vault

SST

$=$ Liquid Effluent Retention Facility.

224-T TRUSAF

NA

$=$ Not applicable.

TSD

= Plutonium-Uranium Extraction (Facility)

$=$ Radiochemical Engineering Cells

$=$ Single-shell tank.

$=$ Polychlorinated biphenyl

$=$ Transuranic Waste Storage and Assay Facility.

$=$ Treatment, storage, and disposal. 
Table 2-5. Stored Waste Characteristics. (sheet 1 of 2)

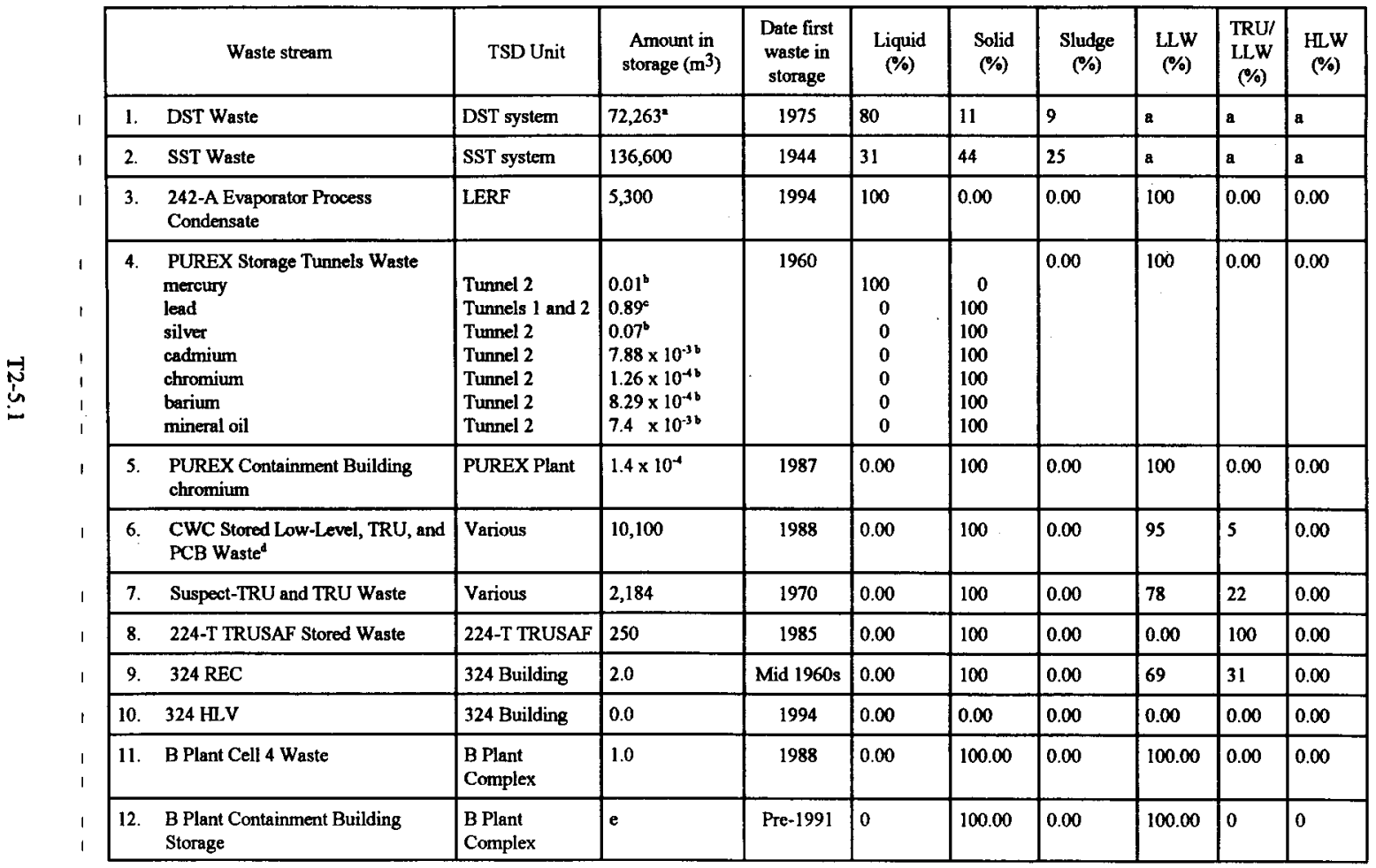


Table 2-5. Stored Waste Characteristics. (sheet 2 of 2)

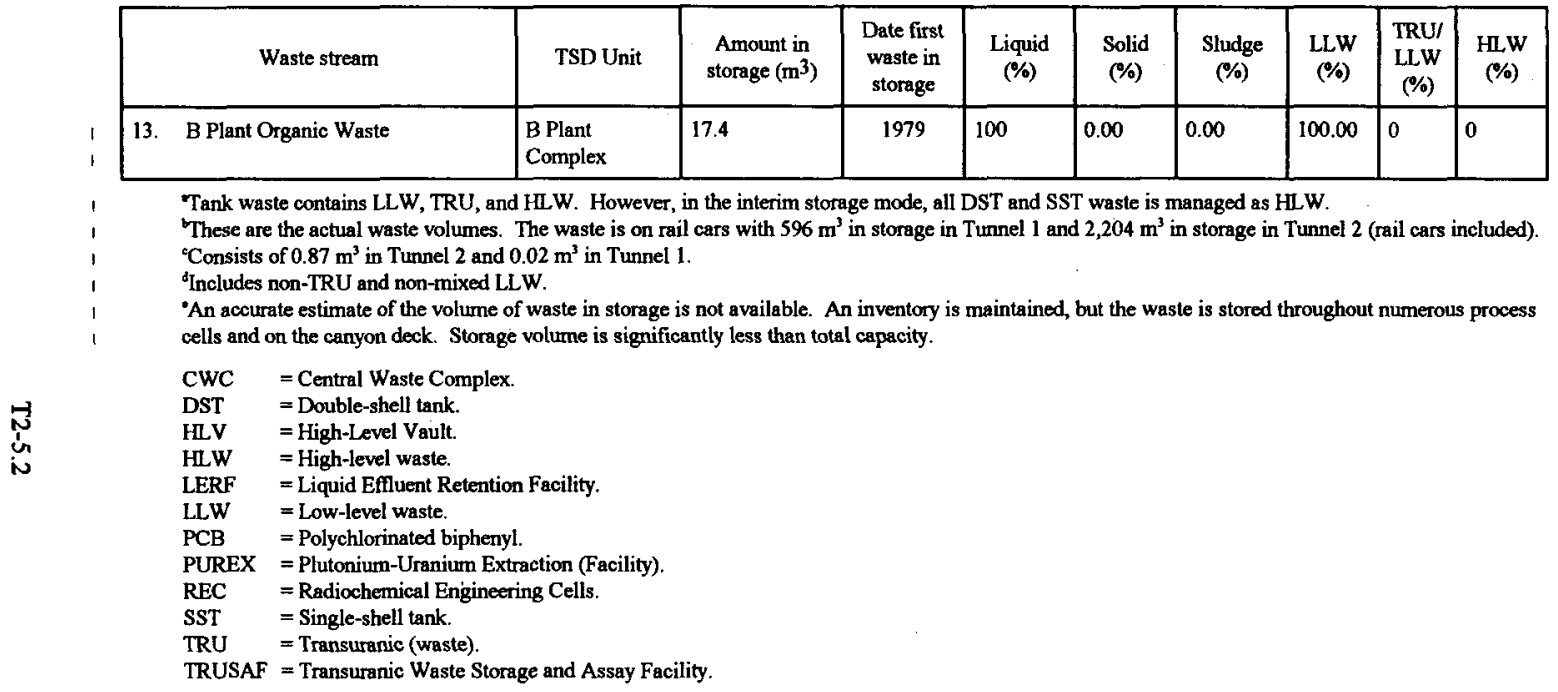


Table 2-6. Treatment of Land Disposal Restricted Waste for Disposal. (sheet 1 of 12)

\begin{tabular}{|c|c|c|c|c|c|c|}
\hline $\begin{array}{l}\text { Waste } \\
\text { codes }\end{array}$ & $\begin{array}{l}\text { Required } \\
\text { treatment }^{2}\end{array}$ & $\begin{array}{c}\text { Planned } \\
\text { treatment }\end{array}$ & $\begin{array}{l}\text { Treatment } \\
\text { facility }\end{array}$ & $\begin{array}{l}\text { Facility } \\
\text { capacity } \\
\left(\mathrm{m}^{3} / \text { day }\right)\end{array}$ & $\begin{array}{l}\text { Disposal } \\
\text { facility }\end{array}$ & $\begin{array}{l}\text { Treat- } \\
\text { ment } \\
\text { date }\end{array}$ \\
\hline \multicolumn{7}{|c|}{$\begin{array}{l}\text { 1. DST Waste (includes NCAW, NCRW, complex concentrate, and PFP waste) } \\
\text { (low-activity fraction) }\end{array}$} \\
\hline F001 & $6.0 \mathrm{mg} / \mathrm{kg}$ & vitrification & $\mathrm{TBD}^{\mathrm{c}}$ & TBD & TBD (onsite) & 2005 \\
\hline F002 & $30 \mathrm{mg} / \mathrm{kg}$ & vitrification & $\mathrm{TBD}^{\mathrm{c}}$ & TBD & TBD (onsite) & 2005 \\
\hline F003 & multiple & vitrification & $\mathrm{TBD}^{\mathrm{c}}$ & TBD & TBD (onsite) & 2005 \\
\hline F004 & $5.6 \mathrm{mg} / \mathrm{kg}$ & vitrification & $\mathrm{TBD}^{\mathrm{c}}$ & TBD & TBD (onsite) & 2005 \\
\hline F005 & $36 \mathrm{mg} / \mathrm{kg}$ & vitrification & $\mathrm{TBD}^{\mathrm{c}}$ & TBD & TBD (onsite) & 2005 \\
\hline F039 & multiple & vitrification & $\mathrm{TBD}^{\mathrm{c}}$ & TBD & TBD (onsite) & 2005 \\
\hline D001 & $\begin{array}{l}\text { deactivation and } \\
\text { meet } \\
40 \text { CFR } 268.48\end{array}$ & vitrification & $\mathrm{TBD}^{\mathrm{C}}$ & TBD & TBD (onsite) & 2005 \\
\hline D002 & $\begin{array}{l}\text { deactivation and } \\
\text { meet } \\
40 \text { CFR } 268.48\end{array}$ & vitrification & $\mathrm{TBD}^{\mathrm{c}}$ & TBD & TBD (onsite) & 2005 \\
\hline D003 & $\begin{array}{l}\text { deactivation and } \\
\text { meet } \mathrm{CN} \\
\text { concentration } \\
\text { standards }\end{array}$ & vitrification & $\mathrm{TBD}^{\mathrm{c}}$ & TBD & TBD (onsite) & 2005 \\
\hline D004 & $5.0 \mathrm{mg} / \mathrm{L}$ & vitrification & TBD $^{c}$ & TBD & TBD (onsite) & 2005 \\
\hline D005 & $100 \mathrm{mg} / \mathrm{L}$ & vitrification & $\mathrm{TBD}^{\mathrm{c}}$ & TBD & TBD (onsite) & 2005 \\
\hline D006 & $1.0 \mathrm{mg} / \mathrm{L}$ & vitrification & $\mathrm{TBD}^{\mathrm{c}}$ & TBD & TBD (onsite) & 2005 \\
\hline D007 & $5.0 \mathrm{mg} / \mathrm{L}$ & vitrification & $\mathrm{TBD}^{\mathrm{c}}$ & TBD & TBD (onsite) & 2005 \\
\hline D008 & $5.0 \mathrm{mg} / \mathrm{L}$ & vitrification & $\mathrm{TBD}^{\mathrm{c}}$ & TBD & TBD (onsite) & 2005 \\
\hline D009 & $0.2 \mathrm{mg} / \mathrm{L}$ & vitrification & $\mathrm{TBD}^{\mathrm{c}}$ & TBD & TBD (onsite) & 2005 \\
\hline D010 & $5.7 \mathrm{mg} / \mathrm{L}$ & vitrification & $\mathrm{TBD}^{\mathrm{c}}$ & TBD & TBD (onsite) & 2005 \\
\hline D011 & $5.0 \mathrm{mg} / \mathrm{L}$ & vitrification & TBD $^{c}$ & TBD & TBD (onsite) & 2005 \\
\hline WT01 & none $\mathrm{e}^{\mathrm{j}}$ & vitrification & $\mathrm{TBD}^{\mathrm{c}}$ & TBD & TBD (onsite) & 2005 \\
\hline WT02 & none & vitrification & $\mathrm{TBD}^{\mathrm{c}}$ & TBD & TBD (onsite) & 2005 \\
\hline
\end{tabular}


Table 2-6. Treatment of Land Disposal Restricted Waste for Disposal. (sheet 2 of 12)

\begin{tabular}{|c|c|c|c|c|c|c|}
\hline $\begin{array}{l}\text { Waste } \\
\text { codes }\end{array}$ & $\begin{array}{l}\text { Required } \\
\text { treatment }^{2}\end{array}$ & $\begin{array}{c}\text { Planned } \\
\text { treatment }\end{array}$ & $\begin{array}{c}\text { Treatment } \\
\text { facility }\end{array}$ & $\begin{array}{l}\text { Facility } \\
\text { capacity } \\
\left(\mathrm{m}^{3} / \text { day }\right)\end{array}$ & $\begin{array}{l}\text { Disposal } \\
\text { facility }\end{array}$ & $\begin{array}{l}\text { Treat- } \\
\text { ment } \\
\text { date }\end{array}$ \\
\hline WP01 & none & vitrification & TBD $^{e}$ & TBD & TBD (onsite) & 2005 \\
\hline WP02 & none & vitrification & $\mathrm{TBD}^{\mathrm{c}}$ & TBD & TBD (onsite) & 2005 \\
\hline \multicolumn{7}{|c|}{ Pretreated Complexed Concentrate Waste (high-level fraction) } \\
\hline F001 & $6.0 \mathrm{mg} / \mathrm{kg}$ & vitrification & TBD $^{\mathrm{c}}$ & TBD & repository & 2009 \\
\hline F002 & $30 \mathrm{mg} / \mathrm{kg}$ & vitrification & TBD $^{c}$ & TBD & repository & 2009 \\
\hline F003 & multiple & vitrification & TBD $^{c}$ & TBD & repository & 2009 \\
\hline F004 & $5.6 \mathrm{mg} / \mathrm{kg}$ & vitrification & TBD $^{c}$ & TBD & repository & 2009 \\
\hline F005 & $36 \mathrm{mg} / \mathrm{kg}$ & vitrification & $\mathrm{TBD}^{\mathrm{c}}$ & TBD & repository & 2009 \\
\hline F039 & multiple & vitrification & $\mathrm{TBD}^{\mathrm{c}}$ & TBD & repository & 2009 \\
\hline D001 & $\begin{array}{l}\text { deactivation and } \\
\text { meet } \\
40 \text { CFR } 268.48\end{array}$ & vitrification & $\mathrm{TBD}^{\mathrm{c}}$ & TBD & repository & 2009 \\
\hline D002 & HLVIT & vitrification & $\mathrm{TBD}^{\mathrm{c}}$ & TBD & repository & 2009 \\
\hline D003 & $\begin{array}{l}\text { deactivation and } \\
\text { meet } \mathrm{CN} \\
\text { concentration } \\
\text { standards }\end{array}$ & vitrification & $\mathrm{TBD}^{\mathrm{c}}$ & TBD & repository & 2009 \\
\hline D004 & HLVIT & vitrification & $\mathrm{TBD}^{\mathrm{c}}$ & TBD & repository & 2009 \\
\hline D005 & HLVIT & vitrification & $\mathrm{TBD}^{\mathrm{c}}$ & TBD & repository & 2009 \\
\hline D006 & HLVIT & vitrification & $\mathrm{TBD}^{\mathrm{c}}$ & TBD & repository & 2009 \\
\hline D007 & HLVIT & vitrification & $\mathrm{TBD}^{\mathrm{c}}$ & TBD & repository & 2009 \\
\hline D008 & HLVIT & vitrification & $\mathrm{TBD}^{\mathrm{c}}$ & TBD & repository & 2009 \\
\hline D009 & HLVIT & vitrification & $\mathrm{TBD}^{\mathrm{c}}$ & TBD & repository & 2009 \\
\hline D010 & HLVIT & vitrification & $\mathrm{TBD}^{\mathrm{c}}$ & TBD & repository & 2009 \\
\hline D011 & HLVIT & vitrification & $\mathrm{TBD}^{\mathrm{c}}$ & TBD & repository & 2009 \\
\hline WT01 & none $e^{j}$ & vitrification & TBD $^{\circ}$ & TBD & repository & 2009 \\
\hline WT02 & none & vitrification & TBD $^{c}$ & TBD & repository & 2009 \\
\hline
\end{tabular}


Table 2-6. Treatment of Land Disposal Restricted Waste for Disposal. (sheet 3 of 12)

\begin{tabular}{|c|c|c|c|c|c|c|}
\hline $\begin{array}{l}\text { Waste } \\
\text { codes }\end{array}$ & $\begin{array}{l}\text { Required } \\
\text { treatment }^{\mathrm{a}}\end{array}$ & $\begin{array}{l}\text { Planned } \\
\text { treatment }\end{array}$ & $\begin{array}{l}\text { Treatment } \\
\text { facility }\end{array}$ & $\begin{array}{l}\text { Facility } \\
\text { capacity } \\
\left(\mathrm{m}^{3} / \text { day }\right)\end{array}$ & $\begin{array}{l}\text { Disposal } \\
\text { facility }\end{array}$ & $\begin{array}{l}\text { Treat- } \\
\text { ment } \\
\text { date }\end{array}$ \\
\hline WP01 & none' & vitrification & $\mathrm{TBD}^{\mathrm{c}}$ & TBD & repository & 2009 \\
\hline WP02 & none & vitrification & $\mathrm{TBD}^{\mathrm{c}}$ & TBD & repository & 2009 \\
\hline \multicolumn{7}{|c|}{$\begin{array}{l}\text { 2. SST Waste }{ }^{b, d} \\
\text { High-level fraction }\end{array}$} \\
\hline F001 & $6.0 \mathrm{mg} / \mathrm{kg}$ & vitrification & $\mathrm{TBD}^{\mathrm{c}}$ & TBD & repository & 2009 \\
\hline F002 & $30 \mathrm{mg} / \mathrm{kg}$ & vitrification & $\mathrm{TBD}^{\mathrm{c}}$ & TBD & repository & 2009 \\
\hline F003 & multiple & vitrification & $\mathrm{TBD}^{\mathrm{c}}$ & TBD & repository & 2009 \\
\hline F004 & $5.6 \mathrm{mg} / \mathrm{kg}$ & vitrification & $\mathrm{TBD}^{\mathrm{c}}$ & TBD & repository & 2009 \\
\hline F005 & $36 \mathrm{mg} / \mathrm{kg}$ & vitrification & TBD $^{\circ}$ & TBD & repository & 2009 \\
\hline D001 & $\begin{array}{l}\text { deactivation and } \\
\text { meet } \\
40 \text { CFR } 268.48\end{array}$ & vitrification & $\mathrm{TBD}^{\mathrm{c}}$ & TBD & repository & 2009 \\
\hline D002 & HLVIT & vitrification & TBD $^{\mathrm{c}}$ & TBD & repository & 2009 \\
\hline D003 & $\begin{array}{l}\text { deactivation and } \\
\text { meet } \mathrm{CN} \\
\text { concentration } \\
\text { standards }\end{array}$ & vitrification & $\mathrm{TBD}^{\mathrm{c}}$ & TBD & repository & 2009 \\
\hline D004 & HLVIT & vitrification & $\mathrm{TBD}^{\mathrm{e}}$ & TBD & repository & 2009 \\
\hline D005 & HLVIT & vitrification & $\mathrm{TBD}^{\mathrm{c}}$ & TBD & repository & 2009 \\
\hline D006 & HLVTT & vitrification & TBD $^{\mathrm{c}}$ & TBD & repository & 2009 \\
\hline D007 & HL VIT & vitrification & TBD $^{\mathrm{c}}$ & TBD & repository & 2009 \\
\hline D008 & HLVIT & vitrification & TBD $^{c}$ & TBD & repository & 2009 \\
\hline D009 & HLVIT & vitrification & TBD $^{\mathrm{c}}$ & TBD & repository & 2009 \\
\hline D010 & HLVIT & vitrification & TBD $^{\mathrm{c}}$ & TBD & repository & 2009 \\
\hline D011 & HLVIT & vitrification & TBD $^{\mathrm{c}}$ & TBD & repository & 2009 \\
\hline \multicolumn{7}{|c|}{ Low-activity fraction } \\
\hline F001 & $6.0 \mathrm{mg} / \mathrm{kg}$ & vitrification & TBD $^{\mathrm{c}}$ & TBD & TBD (onsite) & 2005 \\
\hline
\end{tabular}


Table 2-6. Treatment of Land Disposal Restricted Waste

for Disposal. (sheet 4 of 12)

\begin{tabular}{|c|c|c|c|c|c|c|}
\hline $\begin{array}{l}\text { Waste } \\
\text { codes }\end{array}$ & $\begin{array}{l}\text { Required } \\
\text { treatment }^{*}\end{array}$ & $\begin{array}{l}\text { Planned } \\
\text { treatment }\end{array}$ & $\begin{array}{c}\text { Treatment } \\
\text { facility }\end{array}$ & $\begin{array}{l}\text { Facility } \\
\text { capacity } \\
\left(\mathrm{m}^{3} / \text { day }\right)\end{array}$ & $\begin{array}{l}\text { Disposal } \\
\text { facility }\end{array}$ & $\begin{array}{c}\text { Treat- } \\
\text { ment } \\
\text { date }\end{array}$ \\
\hline F002 & $30 \mathrm{mg} / \mathrm{kg}$ & vitrification & $\mathrm{TBD}^{\mathrm{c}}$ & TBD & TBD (onsite) & 2005 \\
\hline F003 & multiple & vitrification & TBD $^{c}$ & TBD & TBD (onsite) & 2005 \\
\hline F004 & $5.6 \mathrm{mg} / \mathrm{kg}$ & vitrification & $\mathrm{TBD}^{\mathrm{c}}$ & TBD & TBD (onsite) & 2005 \\
\hline F005 & $36 \mathrm{mg} / \mathrm{kg}$ & vitrification & TBD $^{c}$ & TBD & TBD (onsite) & 2005 \\
\hline D001 & $\begin{array}{l}\text { deactivation and } \\
\text { meet } \\
40 \text { CFR } 268.48\end{array}$ & vitrification & $\mathrm{TBD}^{\mathrm{C}}$ & TBD & TBD (onsite) & 2005 \\
\hline D002 & $\begin{array}{l}\text { deactivation and } \\
\text { meet } \\
40 \text { CFR } 268.48\end{array}$ & vitrification & $\mathrm{TBD}^{\mathrm{c}}$ & TBD & TBD (onsite) & 2005 \\
\hline D003 & $\begin{array}{l}\text { deactivation and } \\
\text { meet } \mathrm{CN} \\
\text { concentration } \\
\text { standards }\end{array}$ & vitrification & $\mathrm{TBD}^{\mathrm{c}}$ & TBD & TBD (onsite) & 2005 \\
\hline D004 & $5.0 \mathrm{mg} / \mathrm{L}$ & vitrification & $\mathrm{TBD}^{\mathrm{c}}$ & TBD & TBD (onsite) & 2005 \\
\hline D005 & $100 \mathrm{mg} / \mathrm{L}$ & vitrification & $\mathrm{TBD}^{\mathrm{c}}$ & TBD & TBD (onsite) & 2005 \\
\hline D006 & $1.0 \mathrm{mg} / \mathrm{L}$ & vitrification & $\mathrm{TBD}^{\mathrm{c}}$ & TBD & TBD (onsite) & 2005 \\
\hline D007 & $5.0 \mathrm{mg} / \mathrm{L}$ & vitrification & TBD $^{c}$ & TBD & TBD (onsite) & 2005 \\
\hline D008 & $5.0 \mathrm{mg} / \mathrm{L}$ & vitrification & TBD $^{c}$ & TBD & TBD (onsite) & 2005 \\
\hline D009 & thermal & vitrification & $\mathrm{TBD}^{\mathrm{c}}$ & TBD & TBD (onsite) & 2005 \\
\hline D010 & $5.7 \mathrm{mg} / \mathrm{L}$ & vitrification & TBD $^{c}$ & TBD & TBD (onsite) & 2005 \\
\hline D011 & $5.0 \mathrm{mg} / \mathrm{L}$ & vitrification & $\mathrm{TBD}^{\mathrm{c}}$ & TBD & TBD (onsite) & 2005 \\
\hline \multicolumn{7}{|c|}{ 3. 242-A Evaporator Process Condensate } \\
\hline F001 & $N A^{k}$ & $\begin{array}{l}\text { destruction/ } \\
\text { delisting }\end{array}$ & ETF & $600^{4}$ & SALDS & 1995 \\
\hline F002 & $\mathrm{NA}^{\mathrm{k}}$ & $\begin{array}{l}\text { destruction/ } \\
\text { delisting }\end{array}$ & ETF & $600^{1}$ & SALDS & 1995 \\
\hline F003 & $\mathrm{NA}^{\mathrm{k}}$ & $\begin{array}{l}\text { destruction/ } \\
\text { delisting }\end{array}$ & ETF & $600^{1}$ & SALDS & 1995 \\
\hline
\end{tabular}


Table 2-6. Treatment of Land Disposal Restricted Waste for Disposal. (sheet 5 of 12)

\begin{tabular}{|c|c|c|c|c|c|c|}
\hline $\begin{array}{l}\text { Waste } \\
\text { codes }\end{array}$ & $\begin{array}{l}\text { Required } \\
\text { treatment }\end{array}$ & $\begin{array}{l}\text { Planned } \\
\text { treatment }\end{array}$ & $\begin{array}{l}\text { Treatment } \\
\text { facility }\end{array}$ & $\begin{array}{l}\text { Facility } \\
\text { capacity } \\
\left(\mathrm{m}^{3} / \text { day }\right)\end{array}$ & $\begin{array}{l}\text { Disposal } \\
\text { facility }\end{array}$ & $\begin{array}{c}\text { Treat- } \\
\text { ment } \\
\text { date }\end{array}$ \\
\hline F004 & $N A^{k}$ & $\begin{array}{l}\text { destruction/ } \\
\text { delisting }\end{array}$ & ETF & $600^{1}$ & SALDS & 1995 \\
\hline F005 & $\mathrm{NA}^{\mathrm{k}}$ & $\begin{array}{l}\text { destruction/ } \\
\text { delisting }\end{array}$ & ETF & $600^{1}$ & SALDS & 1995 \\
\hline \multicolumn{7}{|c|}{ 4. PUREX Storage Tunnels Waste } \\
\hline D001 & $\begin{array}{l}\text { deactivation and } \\
\text { meet } \\
40 \text { CFR } 268.48\end{array}$ & TBD & TBD & TBD & TBD & TBD \\
\hline D005 & $100 \mathrm{mg} / \mathrm{h}$ & TBD & TBD & TBD & TBD & TBD \\
\hline D006 & $1.0 \mathrm{mg} / \mathrm{L}$ & TBD & TBD & TBD & TBD & TBD \\
\hline D007 & $5.0 \mathrm{mg} / \mathrm{L}$ & TBD & TBD & TBD & TBD & TBD \\
\hline D008 & $\begin{array}{l}\text { macro- } \\
\text { encapsulation }\end{array}$ & TBD & TBD & TBD & TBD & TBD \\
\hline D009 & amalgamation & TBD & TBD & TBD & TBD & TBD \\
\hline D011 & $5.0 \mathrm{mg} / \mathrm{L}$ & TBD & TBD & TBD & TBD & TBD \\
\hline WT02 & none $^{j}$ & TBD & TBD & TBD & TBD & TBD \\
\hline \multicolumn{7}{|c|}{ 5. PUREX Containment Building } \\
\hline D007 & $5.0 \mathrm{mg} / \mathrm{L}$ & TBD & TBD & TBD & TBD & TBD \\
\hline WT01 & none & TBD & TBD & TBD & TBD & TBD \\
\hline \multicolumn{7}{|c|}{$\begin{array}{l}\text { 6. CWC Stored Low-Level, Transuranic, and PCB Waste } \\
\text { Low-level waste }\end{array}$} \\
\hline F001 & multiple & Various $^{m}$ & Various $^{\mathrm{n}}$ & TBD & MWDF & $1999^{\mathrm{e}, \mathrm{h}}$ \\
\hline F002 & multiple & Various $^{\mathrm{m}}$ & Various $^{\mathrm{n}}$ & TBD & MWDF & $1999^{\mathrm{chh}}$ \\
\hline F003 & multiple & Various $^{\mathrm{m}}$ & Various $^{n}$ & TBD & MWDF & $1999^{\mathrm{eh}}$ \\
\hline F004 & multiple & Various $^{\mathrm{m}}$ & Various $^{n}$ & TBD & MWDF & $1999^{\mathrm{e}, \mathrm{h}}$ \\
\hline F005 & multiple & Various $^{\mathbf{m}}$ & Various $^{\mathrm{n}}$ & TBD & MWDF & $1999^{\mathrm{c}, \mathrm{h}}$ \\
\hline F039 & multiple & TBD & TBD & TBD & TBD & TBD \\
\hline
\end{tabular}


Table 2-6. Treatment of Land Disposal Restricted Waste for Disposal. (sheet 6 of 12)

\begin{tabular}{|c|c|c|c|c|c|c|}
\hline $\begin{array}{l}\text { Waste } \\
\text { codes }\end{array}$ & $\begin{array}{l}\text { Required } \\
\text { treatment" }\end{array}$ & $\begin{array}{l}\text { Planned } \\
\text { treatment }\end{array}$ & $\begin{array}{c}\text { Treatment } \\
\text { facility }\end{array}$ & $\begin{array}{l}\text { Facility } \\
\text { capacity } \\
\left(\mathrm{m}^{3} / \text { day }\right)\end{array}$ & $\begin{array}{l}\text { Disposal } \\
\text { facility }\end{array}$ & $\begin{array}{l}\text { Treat- } \\
\text { ment } \\
\text { date }\end{array}$ \\
\hline D001 & $\begin{array}{l}\text { deactivation and } \\
\text { meet } \\
40 \text { CFR } 268.48\end{array}$ & various $^{m}$ & various $^{n}$ & TBD & TBD & $1999^{\circ}$ \\
\hline D002 & $\begin{array}{l}\text { deactivation and } \\
\text { meet } \\
40 \text { CFR } 268.48\end{array}$ & various $^{m}$ & various $^{n}$ & TBD & TBD & $1999^{\circ}$ \\
\hline D003 & $\begin{array}{l}\text { deactivation and } \\
\text { meet } \mathrm{CN} \\
\text { concentration } \\
\text { standards }\end{array}$ & various ${ }^{m}$ & various $^{n}$ & TBD & TBD & $1999^{\mathrm{e}, \mathrm{h}}$ \\
\hline D004 & $5.0 \mathrm{mg} / \mathrm{L}$ & various $^{\mathrm{m}}$ & various $^{n}$ & TBD & TBD & $1999^{\mathrm{c}, \mathrm{h}}$ \\
\hline D005 & $100 \mathrm{mg} / \mathrm{L}$ & various $^{m}$ & various $^{n}$ & TBD & TBD & $1999^{\text {e,h }}$ \\
\hline D006 & $1.0 \mathrm{mg} / \mathrm{L}$ & various $^{\mathrm{m}}$ & various ${ }^{n}$ & TBD & TBD & $1999^{\mathrm{chh}}$ \\
\hline D007 & $5.0 \mathrm{mg} / \mathrm{L}$ & various $^{\mathrm{m}}$ & various ${ }^{n}$ & TBD & TBD & $1999^{\mathrm{c}, \mathrm{h}}$ \\
\hline D008 & $\begin{array}{l}5.0 \mathrm{mg} / \mathrm{L} \text { and } \\
\text { macro- } \\
\text { encapsulation }\end{array}$ & various $^{\mathrm{m}}$ & various $^{n}$ & TBD & TBD & $1999^{\mathrm{e,h}}$ \\
\hline D009 & amalgamation & various $^{m}$ & various $^{n}$ & TBD & TBD & $1999^{\mathrm{ehh}}$ \\
\hline D010 & $5.7 \mathrm{mg} / \mathrm{L}$ & various $^{\mathrm{m}}$ & various $^{n}$ & TBD & TBD & $1999^{\text {e,h }}$ \\
\hline D011 & $5.0 \mathrm{mg} / \mathrm{L}$ & various $^{\text {m }}$ & various $^{n}$ & TBD & TBD & $1999^{\mathrm{e,h}}$ \\
\hline D012 & $0.13 \mathrm{mg} / \mathrm{kg}$ & $\begin{array}{l}\text { thermal } \\
\text { treatment }\end{array}$ & CTT & TBD & TBD & $2000^{\mathrm{h}}$ \\
\hline D016 & $10 \mathrm{mg} / \mathrm{kg}$ & \begin{tabular}{|l} 
thermal \\
treatment
\end{tabular} & CTT & TBD & TBD & $2000^{h}$ \\
\hline D039 & $6.0 \mathrm{mg} / \mathrm{kg}$ & $\begin{array}{l}\text { thermal } \\
\text { treatment }\end{array}$ & CTT & TBD & TBD & $2000^{h}$ \\
\hline WT01 & none & NA & NA & NA & MWDF & NA \\
\hline WT02 & none & NA & NA & NA & MWDF & NA \\
\hline WP01 & none ${ }^{j}$ & $\mathrm{NA}$ & NA & NA & MWDF & NA \\
\hline WP02 & none & NA & NA & NA & MWDF & NA \\
\hline
\end{tabular}


Table 2-6. Treatment of Land Disposal Restricted Waste for Disposal. (sheet 7 of 12)

\begin{tabular}{|c|c|c|c|c|c|c|}
\hline $\begin{array}{l}\text { Waste } \\
\text { codes }\end{array}$ & $\begin{array}{l}\text { Required } \\
\text { treatment }\end{array}$ & $\begin{array}{l}\text { Planned } \\
\text { treatment }\end{array}$ & $\begin{array}{l}\text { Treatment } \\
\text { facility }\end{array}$ & $\begin{array}{l}\text { Facility } \\
\text { capacity } \\
\left(\mathrm{m}^{3} / \text { day }\right)\end{array}$ & $\begin{array}{l}\text { Disposal } \\
\text { facility }\end{array}$ & $\begin{array}{c}\text { Treat- } \\
\text { ment } \\
\text { date }\end{array}$ \\
\hline U080 & $30 \mathrm{mg} / \mathrm{kg}$ & various $^{m}$ & Various & TBD & MWDF & $1999^{\circ}$ \\
\hline U123 & $\begin{array}{l}\text { combustion or } \\
\text { stabilization }^{q}\end{array}$ & $\begin{array}{l}\text { combustion or } \\
\text { stabilization }^{q}\end{array}$ & CST or DMT & TBD & MWDF & $\mathrm{TBD}^{\mathrm{h}}$ \\
\hline U161 & $33 \mathrm{mg} / \mathrm{kg}$ & various $^{\mathbf{m}}$ & Various & TBD & MWDF & $1999^{\circ}$ \\
\hline P029 & multiple & stabilization & CST or DMT & TBD & MWDF & $1999^{\circ}$ \\
\hline P030 & multiple & stabilization & CST or DMT & TBD & MWDF & $1999^{e}$ \\
\hline P098 & multiple & stabilization & CST or DMT & TBD & MWDF & $1999^{\circ}$ \\
\hline P106 & multiple & stabilization & CST or DMT & TBD & MWDF & $1999^{\circ}$ \\
\hline P120 & stabilization & stabilization & CST or DMT & TBD & MWDF & $1999^{\circ}$ \\
\hline W001 & incineration & $\begin{array}{l}\text { thermal } \\
\text { treatment }\end{array}$ & CTT & TBD & MWDF & $2000^{h}$ \\
\hline & . & & suranic waste & & & \\
\hline F003 & none $^{f}$ & none & WRAP $1^{\text {I }}$ & TBD & WIPP & 1998 \\
\hline F005 & none $^{f}$ & none $^{i}$ & WRAP $1^{\text {I }}$ & TBD & WIPP & 1998 \\
\hline D001 & none $^{f}$ & none $^{\mathrm{i}}$ & WRAP $1^{I}$ & TBD & WIPP & 1998 \\
\hline D002 & none $^{f}$ & none $e^{i}$ & WRAP $1^{1}$ & TBD & WIPP & 1998 \\
\hline D006 & none $^{f}$ & none $e^{i}$ & WRAP $1^{I}$ & TBD & WIPP & 1998 \\
\hline D007 & none $^{\mathrm{f}}$ & none $e^{i}$ & WRAP $1^{\text {I }}$ & TBD & WIPP & 1998 \\
\hline D008 & none $^{f}$ & none $^{\mathrm{i}}$ & WRAP $1^{\text {I }}$ & TBD & WIPP & 1998 \\
\hline D009 & none $^{t}$ & none $^{\mathrm{i}}$ & WRAP $1^{1}$ & TBD & WIPP & 1998 \\
\hline WT01 & none $^{f}$ & none $^{\mathrm{i}}$ & WRAP $1^{{ }^{1}}$ & TBD & WIPP & 1998 \\
\hline WT02 & none $^{f}$ & none $^{\mathrm{i}}$ & WRAP I I & TBD & WIPP & 1998 \\
\hline W001 & none $^{f}$ & none $^{\mathrm{i}}$ & WRAP $1^{1}$ & TBD & WIPP & 1998 \\
\hline \multicolumn{7}{|c|}{$\begin{array}{l}\text { 7. Suspect-Transuranic and Transuranic Waste } \\
\text { Low-level waste (suspect-transuranic) }\end{array}$} \\
\hline F001 & multiple & various $^{m}$ & various $^{n}$ & TBD & MWDF & $1999^{e, h}$ \\
\hline
\end{tabular}


Table 2-6. Treatment of Land Disposal Restricted Waste for Disposal. (sheet 8 of 12)

\begin{tabular}{|c|c|c|c|c|c|c|}
\hline $\begin{array}{l}\text { Waste } \\
\text { codes }\end{array}$ & $\begin{array}{l}\text { Required } \\
\text { treatment }^{2}\end{array}$ & $\begin{array}{l}\text { Planned } \\
\text { treatment }\end{array}$ & $\begin{array}{c}\text { Treatment } \\
\text { facility }\end{array}$ & $\begin{array}{l}\text { Facility } \\
\text { capacity } \\
\left(\mathrm{m}^{3} / \text { day }\right)\end{array}$ & $\begin{array}{l}\text { Disposal } \\
\text { facility }\end{array}$ & $\begin{array}{l}\text { Treat- } \\
\text { ment } \\
\text { date }\end{array}$ \\
\hline F003 & multiple & various $^{\mathrm{m}}$ & various" & TBD & MWDF & $1999^{\text {a,h }}$ \\
\hline F005 & multiple & various $^{\mathrm{m}}$ & various $^{n}$ & TBD & MWDF & $1999^{\text {e,h }}$ \\
\hline D001 & $\begin{array}{l}\text { deactivation and } \\
\text { meet } \\
40 \text { CFR } 268.48\end{array}$ & various $^{m}$ & various ${ }^{n}$ & TBD & MWDF & $1999^{\mathrm{e}, \mathrm{h}}$ \\
\hline D003 & $\begin{array}{l}\text { deactivation and } \\
\text { meet } \\
40 \text { CFR } 268.48\end{array}$ & various $^{\mathrm{m}}$ & various ${ }^{n}$ & TBD & MWDF & $1999^{\mathrm{c}, \mathrm{h}}$ \\
\hline D005 & $100 \mathrm{mg} / \mathrm{L}$ & various $^{\mathrm{m}}$ & various $^{n}$ & TBD & MWDF & $1999^{\text {e,h }}$ \\
\hline D006 & $1.0 \mathrm{mg} / \mathrm{L}$ & various $^{m}$ & various $^{n}$ & TBD & MWDF & $1999^{\mathrm{c}, \mathrm{h}}$ \\
\hline D007 & $5.0 \mathrm{mg} / \mathrm{L}$ & various $^{\mathrm{m}}$ & various $^{n}$ & TBD & MWDF & $1999^{\text {chh }}$ \\
\hline D008 & $\begin{array}{l}5.0 \mathrm{mg} / \mathrm{L} \text { and } \\
\text { macro- } \\
\text { encapsulation }\end{array}$ & various $^{\mathrm{m}}$ & various $^{n}$ & TBD & MWDF & $1999^{\text {e.h }}$ \\
\hline D009 & multiple & various $^{\mathrm{m}}$ & CST or DMT & TBD & TBD & $1999^{\mathrm{c}, \mathrm{h}}$ \\
\hline D011 & $5.0 \mathrm{mg} / \mathrm{L}$ & various $^{\mathrm{m}}$ & various $^{n}$ & TBD & TBD & $1999^{\mathrm{e}, \mathrm{h}}$ \\
\hline WT01 & none $\mathrm{e}^{\mathrm{j}}$ & NA & NA & NA & MWDF & NA \\
\hline WT02 & none & NA & NA & NA & MWDF & NA \\
\hline WP01 & none $e^{j}$ & NA & NA & NA & MWDF & NA \\
\hline W001 & incineration & $\begin{array}{l}\text { thermal } \\
\text { treatment }\end{array}$ & CTT & NA & MWDF & $2000^{h}$ \\
\hline \multicolumn{7}{|c|}{ Transuranic waste } \\
\hline D006 & none $^{f}$ & none $^{\mathrm{i}}$ & WRAP $1^{\text {I }}$ & TBD & WIPP & 1998 \\
\hline D008 & none $^{r}$ & none $e^{i}$ & WRAP I ${ }^{\prime}$ & TBD & WIPP & 1998 \\
\hline WT01 & none $^{f}$ & none $^{i}$ & WRAP $1^{\text {I }}$ & TBD & WIPP & 1998 \\
\hline P015 & none $^{f}$ & none $^{i}$ & WRAP $1^{\mathrm{I}}$ & TBD & WIPP & 1998 \\
\hline \multicolumn{7}{|c|}{ 8. 224-T TRUSAF Stored Waste } \\
\hline D002 & none $^{t}$ & none $^{\mathrm{i}}$ & WRAP $1^{\text {I }}$ & TBD & WIPP & 1998 \\
\hline
\end{tabular}


Table 2-6. Treatment of Land Disposal Restricted Waste for Disposal. (sheet 9 of 12)

\begin{tabular}{|c|c|c|c|c|c|c|}
\hline $\begin{array}{l}\text { Waste } \\
\text { codes }\end{array}$ & $\begin{array}{l}\text { Required } \\
\text { treatment }\end{array}$ & $\begin{array}{l}\text { Planned } \\
\text { treatment }\end{array}$ & $\begin{array}{c}\text { Treatment } \\
\text { facility }\end{array}$ & $\begin{array}{l}\text { Facility } \\
\text { capacity } \\
\left(\mathrm{m}^{3} / \text { day) }\right.\end{array}$ & $\begin{array}{l}\text { Disposal } \\
\text { facility }\end{array}$ & $\begin{array}{c}\text { Treat- } \\
\text { ment } \\
\text { date }\end{array}$ \\
\hline D005 & none $e^{f}$ & none $e^{i}$ & WRAP $1^{1}$ & TBD & WIPP & 1998 \\
\hline D006 & none $^{f}$ & none $^{\mathrm{i}}$ & WRAP $1^{1}$ & TBD & WIPP & 1998 \\
\hline D007 & none $e^{f}$ & none $e^{i}$ & WRAP $1^{1}$ & TBD & WIPP & 1998 \\
\hline D008 & none $^{f}$ & none $^{i}$ & WRAP $1^{1}$ & TBD & WIPP & 1998 \\
\hline D009 & none $^{f}$ & none & WRAP $1^{\mathrm{I}}$ & TBD & WIPP & 1998 \\
\hline WP01 & none $^{f}$ & none $^{i}$ & WRAP $1^{I}$ & TBD & WIPP & 1998 \\
\hline WT01 & none $^{r}$ & none $e^{i}$ & WRAP $1^{\text {I }}$ & TBD & WIPP & 1998 \\
\hline \multicolumn{7}{|c|}{ 9. 324 REC } \\
\hline D005 & $100 \mathrm{mg} / \mathrm{L}$ & TBD & TBD & TBD & TBD & TBD \\
\hline D006 & $\begin{array}{l}1.0 \mathrm{mg} / \mathrm{L} \\
\text { macro- } \\
\text { encapsulation }\end{array}$ & TBD & TBD & TBD & TBD & TBD \\
\hline D007 & $5.0 \mathrm{mg} / \mathrm{L}$ & TBD & TBD & TBD & TBD & TBD \\
\hline D008 & $\begin{array}{l}5.0 \mathrm{mg} / \mathrm{L} \text {, } \\
\text { macro- } \\
\text { encapsulation }\end{array}$ & TBD & TBD & TBD & TBD & TBD \\
\hline WT01 & none' & NA & NA & NA & $\mathrm{NA}$ & TBD \\
\hline WT02 & none & NA & NA & NA & NA & TBD \\
\hline \multicolumn{7}{|c|}{$\begin{array}{l}10.324 \mathrm{HLV} \\
\text { Note: This waste has been treated. Residues have been transferred to } 324 \text { REC. }\end{array}$} \\
\hline \multicolumn{7}{|c|}{ 11. B Plant Cell 4 Waste } \\
\hline D008 & $\begin{array}{l}\text { macro- } \\
\text { encapsulation }\end{array}$ & TBD & TBD & TBD & TBD & TBD \\
\hline \multicolumn{7}{|c|}{ 12. B Plant Containment Building Storage } \\
\hline D004 & $5.0 \mathrm{mg} / \mathrm{L}$ & TBD & TBD & TBD & TBD & TBD \\
\hline D005 & $100 \mathrm{mg} / \mathrm{L}$ & TBD & TBD & TBD & TBD & TBD \\
\hline D006 & $1.0 \mathrm{mg} / \mathrm{L}$ & TBD & TBD & TBD & TBD & TBD \\
\hline
\end{tabular}


Table 2-6. Treatment of Land Disposal Restricted Waste for Disposal. (sheet 10 of 12)

\begin{tabular}{|c|c|c|c|c|c|c|}
\hline $\begin{array}{l}\text { Waste } \\
\text { codes }\end{array}$ & $\begin{array}{l}\text { Required } \\
\text { treatment" }\end{array}$ & $\begin{array}{l}\text { Planned } \\
\text { treatment }\end{array}$ & $\begin{array}{l}\text { Treatment } \\
\text { facility }\end{array}$ & $\begin{array}{l}\text { Facility } \\
\text { capacity } \\
\left(\mathrm{m}^{3} / \text { day }\right)\end{array}$ & $\begin{array}{l}\text { Disposal } \\
\text { facility }\end{array}$ & $\begin{array}{l}\text { Treat- } \\
\text { ment } \\
\text { date }\end{array}$ \\
\hline D007 & $5.0 \mathrm{mg} / \mathrm{L}$ & TBD & TBD & TBD & TBD & TBD \\
\hline $\mathrm{D} 008$ & $\begin{array}{l}\text { macro- } \\
\text { encapsulation }\end{array}$ & TBD & TBD & TBD & TBD & TBD \\
\hline D009 & amalgamation & TBD & TBD & TBD & TBD & TBD \\
\hline D010 & $5.7 \mathrm{mg} / \mathrm{L}$ & TBD & TBD & TBD & TBD & TBD \\
\hline D011 & $5.0 \mathrm{mg} / \mathrm{L}$ & TBD & TBD & TBD & TBD & TBD \\
\hline F001 & $6.0 \mathrm{mg} / \mathrm{kg}$ & TBD & TBD & TBD & TBD & TBD \\
\hline F002 & $30 \mathrm{mg} / \mathrm{kg}^{2}$ & TBD & TBD & TBD & TBD & TBD \\
\hline F003 & multiple & TBD & TBD & TBD & TBD & TBD \\
\hline F004 & $5.6 \mathrm{mg} / \mathrm{kg}$ & TBD & TBD & TBD & TBD & TBD \\
\hline F005 & $36 \mathrm{mg} / \mathrm{kg}$ & TBD & TBD & TBD & TBD & TBD \\
\hline WT01 & nonej & NA & NA & NA & NA & NA \\
\hline WT02 & none & NA & NA & NA & NA & NA \\
\hline \multicolumn{7}{|c|}{ 13. B Plant Organic Waste ${ }^{\circ}$} \\
\hline D004 & $5.0 \mathrm{mg} / \mathrm{L}$ & TBD & TBD & TBD & TBD & TBD \\
\hline D005 & $100 \mathrm{mg} / \mathrm{L}$ & TBD & TBD & TBD & TBD & TBD \\
\hline D006 & $1.0 \mathrm{mg} / \mathrm{L}$ & TBD & TBD & TBD & TBD & TBD \\
\hline D007 & $5.0 \mathrm{mg} / \mathrm{L}$ & TBD & TBD & TBD & TBD & TBD \\
\hline D008 & $5.0 \mathrm{mg} / \mathrm{L}$ & TBD & TBD & TBD & TBD & TBD \\
\hline D009 & $0.2 \mathrm{mg} / \mathrm{L}^{\mathrm{p}}$ & TBD & TBD & TBD & TBD & TBD \\
\hline D010 & $5.7 \mathrm{mg} / \mathrm{L}$ & TBD & TBD & TBD & TBD & TBD \\
\hline D011 & $5.0 \mathrm{mg} / \mathrm{kg}$ & TBD & TBD & TBD & TBD & TBD \\
\hline F001 & $6.0 \mathrm{mg} / \mathrm{kg}$ & TBD & TBD & TBD & TBD & TBD \\
\hline F002 & $30 \mathrm{mg} / \mathrm{kg}$ & TBD & TBD & TBD & TBD & TBD \\
\hline
\end{tabular}


Table 2-6. Treatment of Land Disposal Restricted Waste for Disposal. (sheet 11 of 12)

\begin{tabular}{|l|l|l|l|l|l|l|}
\hline $\begin{array}{l}\text { Waste } \\
\text { codes }\end{array}$ & \multicolumn{1}{|c|}{$\begin{array}{c}\text { Required } \\
\text { treatment }\end{array}$} & \multicolumn{1}{|c|}{$\begin{array}{c}\text { Planned } \\
\text { treatment }\end{array}$} & $\begin{array}{c}\text { Treatment } \\
\text { facility }\end{array}$ & $\begin{array}{c}\text { Facility } \\
\text { capacity } \\
\left(\mathrm{m}^{3} / \text { day }\right)\end{array}$ & $\begin{array}{c}\text { Disposal } \\
\text { facility }\end{array}$ & $\begin{array}{c}\text { Treat- } \\
\text { ment } \\
\text { date }\end{array}$ \\
\hline F003 & multiple & TBD & TBD & TBD & TBD & TBD \\
\hline F004 & $5.6 \mathrm{mg} / \mathrm{kg}$ & TBD & TBD & TBD & TBD & TBD \\
\hline F005 & $36 \mathrm{mg} / \mathrm{kg}$ & TBD & TBD & TBD & TBD & TBD \\
\hline
\end{tabular}

1 condensate waste. Some waste must also be treated to standards for underlying hazardous constituents in 40 CFR 268.48.

'The Tri-Party Agreement strategy calls for pretreatment of essentially all waste within DSTs and SSTs, the resulting streams being processed through either a HLW or LAW vitrification TSD Unit. Therefore, the individual streams such as NCAW and NCRW have been combined to simplify the table. The current baseline strategy has TRU waste combined with the HLW fraction after pretreatment and for vitrification. Studies are planned to be done to see if it is feasible to generate a separate TRU stream that will be processed to meet WIPP waste acceptance criteria.

I Vitrification facilities for both the LAW and $\mathrm{HLW}$ fractions resulting from pretreatment have yet to be designed.

${ }^{d}$ Waste will be retrieved from the SSTs to the extent needed for closure.

'Commercial services for treating this waste are available on this date. This waste will be treated based on facility/commercial services operating schedules. The dates shown are for commercial services only.

No LDR treatment is required as WIPP has been granted an exemption from provisions of LDRs under 61 FR 60704.

Only a partial list of waste codes is given (see note for this stream in Table 2-3).

${ }^{\mathrm{h}} \mathrm{A}$ commercial thermal treatment contract has been awarded for low-level mixed waste to support treatment of this waste code.

'WRAP 1 will process waste to meet WIPP waste acceptance criteria.

Mixed extremely hazardous waste may be land-disposed in Washington State in DOE facilities in accordance with Revised Code of Washington 70.105.050(2).

${ }^{\mathrm{x}}$ Treated effluent from the ETF (including process condensate) is not subject to LDRs. Treated effluent must meet delisting levels indicated in 40 CFR 261, Appendix IX for specified constituents.

'Based on the design-basis feed composition for the 242-A Evaporator process condensate treated in the ETF at $570 \mathrm{~L} / \mathrm{min}$ and 72 percent efficiency. The ETF has an output capacity of $1.2 \mathrm{~m}^{3} /$ day of solid waste.

${ }^{m}$ This waste code will be treated by various treatment methods depending on the waste's physical form and hazardous constituent concentration. Treatments include immobilization for debris, thermal treatment for nondebris waste containing the hazardous constituent below the LDR limit. 
Table 2-6. Treatment of Land Disposal Restricted Waste for Disposal. (sheet 12 of 12)

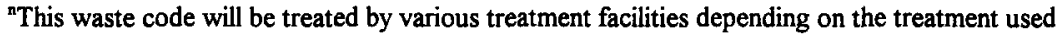
(see Note $\mathrm{m}$ ). Debris waste is scoped to be treated by the Debris Macroencapsulation Treatment (DMT) project, nondebris waste containing the hazardous constituent above the LDR limit is scoped to be treated by the Commercial Thermal Treatment project. Nondebris waste containing the hazardous constituent below the LDR limit is scoped to be treated by the Commercial Stabilization Treatment project.

'Assumes that organic waste is not determined to be HIW. In that case, the required treatment would be HLVIT.

${ }^{P}$ Assumes that characterization of this waste does not determine it to be high-mercury waste.

"An equivalent treatment determination was made by Ecology and EPA for certain 183-H Basin waste to allow stabilization in lieu of combustion on May 12, 1996.

CCW = Constituent concentrations in waste.

CST = Commercial stabilization treatment.

CTT = Commercial thermal treatment.

CWC = Central Waste Complex.

DMT = Debris macroencapsulation treatment.

DST = Double-shell tank.

ETF = Effluent Treatment Facility.

HIVIT $=$ High-level Vitrification.

LLBG = Low-level burial grounds.

LLW = Low-level waste.

LWVP = Low-level waste vitrification plant.

Multiple $=$ Multiple concentration limits (potential multiple constituents within waste code).

MWDF = Hanford mixed waste disposal facility.

PCB = Polychlorinated biphenyl.

PUREX = Plutonium-Uranium Extraction (Facility).

REC = Radiochemical engineering cells.

SALDS = State-approved land disposal structure.

TBD $=$ To be determined.

TRUSAF $=$ Transuranic Waste Storage and Assay Facility.

TSD = Treatment, Storage, and/or Disposal.

WIPP = Waste Isolation Pilot Plant.

WRAP = Waste Receiving and Processing (Facility). 
Table 2-7. Waste Reduction Activities for Hanford Site Land Disposal Mixed Waste. (sheet 1 of 2)

\begin{tabular}{|c|c|c|c|}
\hline Waste & Method to reduce & $\begin{array}{l}\text { Schedule for } \\
\text { implementing waste } \\
\text { reduction } \\
\text { procedures }\end{array}$ & $\begin{array}{l}\text { Projected } \\
\text { waste } \\
\text { reduction }\end{array}$ \\
\hline 1. DST Waste & $\begin{array}{ll}\text { - } & \text { Evaporation } \\
-\quad \text { Minimize frequency of flush } \\
\text { - Minimize flush volumes }\end{array}$ & under way & $80 \%$ \\
\hline 2. SST Waste & $\begin{array}{l}\text { - Waste is no longer being added } \\
\text { to SSTs }\end{array}$ & - & - \\
\hline $\begin{array}{l}\text { 242-A Evapor- } \\
\text { ator Process } \\
\text { Condensate }\end{array}$ & $\begin{array}{l}\text { Effluent Treatment Facility will } \\
\text { remove ammonia, inorganic } \\
\text { salts, metal ions, and destroy } \\
\text { organics } \\
\text { - Recycle a portion of the process } \\
\text { condensate at the } \\
\text { 242-A Evaporator }\end{array}$ & $\begin{array}{l}\text { Complete } \\
\text { (November 1995) }\end{array}$ & $>99 \%$ \\
\hline $\begin{array}{l}\text { 4. PUREX Storage } \\
\text { Tunnels Waste }\end{array}$ & $\begin{array}{l}\text { The tunnels are a permitted } \\
\text { storage facility. Waste is placed } \\
\text { into storage on an as-needed } \\
\text { basis. However, storage of } \\
\text { additional waste resulting from } \\
\text { deactivation activities is not } \\
\text { expected. }\end{array}$ & - & variable \\
\hline $\begin{array}{l}\text { 5. PUREX Contain- } \\
\text { ment Building }\end{array}$ & $\begin{array}{l}\text { - Storage of additional waste as a } \\
\text { result of deactivation activities } \\
\text { is not currently planned. }\end{array}$ & - & variable \\
\hline $\begin{array}{l}\text { 6. CWC, Stored } \\
\text { Low-Level, TRU, } \\
\text { and PCB Waste }\end{array}$ & $\begin{array}{l}\text { - Compaction } \\
\text { - Substitution of nonhazardous } \\
\text { materials } \\
\text { - Neutralization of corrosive } \\
\text { materials } \\
\text { Treatment of waste to } \\
\text { remove hazardous } \\
\text { constituents }\end{array}$ & $\begin{array}{l}\text { Commercial and/or } \\
\text { onsite treatment, Tri- } \\
\text { Party Agreement } \\
\text { Milestones M-91, } \\
\text { M-19 }\end{array}$ & variable \\
\hline $\begin{array}{l}\text { 7. Suspect-TRU and } \\
\text { TRU Waste }\end{array}$ & - Waste is no longer being added & - & - \\
\hline $\begin{array}{l}\text { 8. 224-T TRUSAF } \\
\text { Stored Waste }\end{array}$ & $\begin{array}{l}\text { - Waste is not generated at } \\
\text { TRUSAF }\end{array}$ & - & - \\
\hline
\end{tabular}


Table 2-7. Waste Reduction Activities for Hanford Site Land Disposal Mixed Waste. (sheet 2 of 2)

\begin{tabular}{|c|c|c|c|}
\hline Waste & Method to reduce & $\begin{array}{l}\text { Schedule for } \\
\text { implementing waste } \\
\text { reduction } \\
\text { procedures }\end{array}$ & $\begin{array}{l}\text { Projected } \\
\text { waste } \\
\text { reduction }\end{array}$ \\
\hline 324 REC & $\begin{array}{l}\text { - Waste is no longer being } \\
\text { generated }\end{array}$ & - & - \\
\hline $324 \mathrm{HLV}$ & $\begin{array}{l}\text { Waste was treated to separate } \\
\text { the hazardous constituents. } \\
\text { The resulting residue is being } \\
\text { managed as mixed waste and } \\
\text { the treatment solutions were } \\
\text { disposed of as LLW. }\end{array}$ & - & - \\
\hline $\begin{array}{l}\text { B Plant Cell } 4 \\
\text { Waste }\end{array}$ & $\begin{array}{l}\text { - Clean out and shut down WESF } \\
\text { hot cells }\end{array}$ & underway & $100 \%$ \\
\hline $\begin{array}{l}\text { B Plant } \\
\text { Containment } \\
\text { Building Storage }\end{array}$ & $\begin{array}{l}\text { - Deactivate canyon process } \\
\text { systems, minimize use of } \\
\text { process jumpers }\end{array}$ & underway & unknown \\
\hline $\begin{array}{l}\text { B Plant Organic } \\
\text { Waste }\end{array}$ & $\begin{array}{l}\text { Waste is no longer being } \\
\text { generated }\end{array}$ & - & - \\
\hline
\end{tabular}

'Waste sent to tanks also is reduced at the generating facilities through pretreatment (e.g., destroying ammonia) and recycling of streams.

CWC = Central Waste Complex.

DST = Double-shell tank.

HLV = High-Level Vault.

NA $=$ Not applicable.

PCB = Polychlorinated biphenyl.

PUREX = Plutonium-Uranium Extraction (Facility).

REC =Radiochemical engineering cells.

SST = Single-shell tank.

TBD $=$ To be determined.

TRU = Transuranic.

TRUSAF $=$ Transuranic Waste Storage and Assay Facility.

WESF = Waste Encapsulation and Storage Facility. 


\subsection{INDIVIDUAL WASTE STREAM INFORMATION}

\subsection{DOUBLE-SHELL TANK WASTE}

Most DST system waste was generated during the past production of nuclear materials. The DST waste is stored as alkaline liquids and solids in double-shell underground storage tanks in the 200 Areas of the Hanford Site. Twenty-eight DSTs store 72,263 cubic meters of waste as of December 31, 1996 (PHMC 1997b).

The DST waste is (or has been) generated from the PUREX process, B Plant operations, the PFP, research and development programs, laboratories, and decontamination of plants and equipment. Liquid supematant and interstitial liquids from SSTs also are pumped to DSTs for storage.

Treatment plans are to recover the contents of the tanks, separate the waste into high- and low-activity fractions, and immobilize them for disposal. The TRU and high-level fractions will be vitrified for disposal in a geologic repository; the low activity fraction will be vitrified for disposal near-surface on site.

\subsubsection{Generation}

The DST waste has been generated by operations in the 100,200,300, and 400 Areas of the Hanford Site. The first DSTs were constructed in 1970 and the newest DSTs were completed in 1986. Projected generation rates for DST waste fluctuate depending on the operating schedules of the waste-generating units. The start-up of planned treatment and disposal units will eventually decrease the current and future DST waste volumes.

3.1.1.1 Process. The tanks contain waste from current operations and waste from past chemical separations processes. The major contributors to the waste stored in DSTs are described in the following sections (DOE 1987). All waste streams transferred to the DSTs for storage are treated with sodium hydroxide and sodium nitrite to minimize tank corrosion and to address compatibility issues of waste with the tanks. In addition to newly generated waste, liquid waste stored in SSTs also is transferred to the DSTs. This waste originated from the same sources as that stored in the DSTs, although it physically and chemically differs considerably because of historical evaporation and/or crystallization practices and years of storage. These sources include the PUREX Plant complex, the PFP, and B Plant chemical processes as well as bismuth phosphate separations, uranium recovery, and reduction-oxidation extraction processes. 
Liquid waste streams destined for DSTs from current operations can be classified into four waste categories.

1. Safety--Streams that are required to prevent hazards to personnel or equipment. Examples: PUREX criticality drains must be tested to prevent violation of criticality specifications; B Plant railroad tunnel must be washed down to reduce exposure to personnel.

2. Regulatory--Required by a regulatory body. Example: the aging waste ventilation system condensate could exceed regulatory limits for crib discharge and be sent to DSTs.

3. Tri-Party Agreement--Waste streams that are required to support the Tri-Party Agreement. Examples: Remaining SST wastes are to be pumped to DSTs to meet Tri-Party Agreement milestones for SST stabilization; laboratory wastes are generated from sampling to support Tri-Party Agreement activities.

4. Miscellaneous/Production--Miscellaneous streams in support of Hanford Site program activities. Example: waste generated in cleaning the 400 Area Interim Examination and Maintenance Cell are required to support the fusion program or Argonne National Laboratory.

As a result of the shortage of DST space, waste minimization limits have been set based on Categories 1 through 3 . Category 4 wastes must be reviewed and approved by the Tank Space Management Board for acceptance.

Characterization and waste volume information for both DSTs and SSTs is contained in $A$ History of the 200 Area Tank Farms (WHC 1990e) and monthly waste tank summary documents (PHMC 1997b).

3.1.1.1.1 The PUREX Process. The PUREX Plant began operations in 1956 and operated intermittently until December 1992 when RL decided to deactivate it. The plant received irradiated zirconium-clad fuel from $\mathrm{N}$ Reactor and irradiated aluminum-clad reactor fuel from the Hanford Site single-pass reactors. The cladding was removed from the fuel and the fuel was dissolved in nitric acid. The dissolved fuel was processed through several solvent extraction steps to separate the plutonium, uranium, and neptunium from the fission products contained in the fuel. The three major types of waste generated at PUREX and sent to the DSTs were aging waste, process condensate, and ammonia scrubber feed. The PUREX Plant also sent laboratory waste and flush water to the DSTs.

Aging waste came from the first decontamination solvent extraction column in the PUREX solvent extraction process. The extraction column separated the uranium and TRU products from the majority of the fission products. The fission products were contained in the aqueous nitric acid phase. The aqueous phase was concentrated to recover nitric acid and reduce volumes, then the concentrated stream was sampled. If sample analysis determined the stream to be a waste, it 
was treated with sugar to destroy most of the remaining nitric acid. The waste also was treated with sodium hydroxide and sodium nitrite to meet storage specifications.

The PUREX process condensate stream was generated by condensing vapors from the concentration of the PUREX uranium/nitric acid product and recycle streams. This condensate contained trace quantities of nitric acid. In 1989 the stream was temporarily rerouted to DSTs while its dangerous waste designation was reevaluated. The waste stream was treated to meet tank storage specifications before being transferred.

The PUREX ammonia scrubber feed was generated when water was sprayed to adsorb ammonia gas generated by the decladding and metathesis reactions from the dissolver off gas stream. In late 1987 the ammonium hydroxide concentration in the ammonia scrubber condensate was determined to sometimes exceed 1 percent, making it a dangerous (toxic) waste as designated by state regulations. Therefore it was not appropriate for discharge to a crib. An interim process was established in which ammonia scrubber feed no longer was concentrated for discharge, but was treated for tank storage and transferred as ammonia scrubber waste to underground storage tanks. Treatment consisted of adding sodium hydroxide to adjust the $\mathrm{pH}$ to greater than 12 and adding sodium nitrite to minimize tank corrosion.

No aging waste, PUREX ammonia scrubber feed, or PUREX condensate has been transferred since 1991.

3.1.1.1.2 Plutonium Finishing Plant. In 1949 the PFP began converting plutonium in solution to plutonium metal. This historic waste stream was high in metallic nitrates. The process comprised precipitation, solvent exchange, and ion exchange wastes. The current waste stream generated from the PFP is a low-salt stream from operating the building systems and from laboratory and plutonium stabilization operations. Liquid wastes averaging 4.5 percent solids are sent to DSTs and average about 15 liters per hour. When the facility was operating, similar liquid waste from plutonium reclamation averaged about 270 liters per hour. In October 1996, RL gave direction to complete plutonium stabilization and deactivate the PFP.

3.1.1.1.3 Bismuth Phosphate Separations. Beginning in the early 1940s, B Plant and T Plant separated plutonium from uranium in irradiated fuel by coprecipitation with bismuth phosphate from a uranyl nitrate solution. The plutonium was further separated from fission products by successive precipitation cycles using bismuth phosphate and lanthanum fluoride. Waste containing uranium, acid, and many of the fission products was neutralized and stored in underground SSTs. This separation process was used from 1943 to 1957.

The bismuth phosphate metal wastes were initially stored in separate SSTs; however, the metal waste was reprocessed to recover the uranium and the supernatant was scavenged and disposed to the cribs, leaving very little original metal waste remaining in the SSTs. In addition, through the years waste management operations have created a complex intermingling of the tank wastes. 
3.1.1.1.4 Uranium Recovery Process. Uranium in process waste was mined from the SSTs by sluicing, dissolved in nitric acid, and processed through a solvent extraction process using tributyl phosphate in a kerosene-like solvent. The acid waste from the uranium recovery process was made alkaline and returned to SSTs. The recovery process, which operated from 1952 to 1958 in U Plant and from 1956 to 1958 in PUREX Plant, resulted in an increase in the volume of nonradioactive salts and a small increase in waste volume.

The uranium recovery process operated in U Plant and at the PUREX Plant were similar in that they used tributyl phosphate as the solvent; however, there were significant differences between the two processes. The process in U Plant recovered uranium from bismuth phosphate metal wastes and produced wastes consisting of fission products and residual plutonium. Also, the process in U Plant produced relatively dilute HLW, approximately 19 liters of waste per kilogram of uranium processed. The PUREX Plant process recovered uranium and plutonium, and, at times, neptunium in addition to separating the fission products. The PUREX process produced a much more concentrated high-level waste product, approximately 0.2 liter per kilogram of uranium processed.

No SSTs received acidic wastes or purely nonradioactive salts from these processes. The wastes were all neutral or alkaline in nature and the nonradioactive materials were intimately mixed with radioactive materials.

A significant increase in the volume of waste resulted from the uranium recovery process in U Plant. The process efficiently recovered uranium from the bismuth phosphate metal waste; however, it generated about 2 liters of waste for every liter of bismuth phosphate metal waste processed. This increase in waste volume was the rationale for the ferrocyanide scavenging campaign. It was necessary to reduce the volume of waste in the tanks, and the ferrocyanide scavenging decontaminated the waste sufficiently to enable disposal to the cribs. (Disposal to cribs would not have been allowed by today's standards.)

3.1.1.1.5 Reduction-Oxidation Process. The reduction-oxidation process in the 202-S Plant used a continuous solvent extraction process to extract plutonium and uranium from dissolved fuel in a hexone solvent. The slightly acidic waste stream contained the fission products and large quantities of aluminum nitrate. This waste was neutralized and stored in SSTs. The 202-S Plant operated between 1951 and 1967.

3.1.1.1.6 Cesium and Strontium Recovery. Past operations in B Plant for recovery of cesium and strontium from waste were a main source of DST waste. Waste resulting from the strontium recovery was transferred to complex concentrate tanks. Waste resulting from cesium recovery (cesium raffinate) was segregated and placed in tank 241-AY-101, where it is now known as "dilute complexant waste."

3.1.1.1.7 Other Contributors to Double-Shell Tank Waste. Cleaning solutions and other miscellaneous waste are chemically adjusted to minimize tank corrosivity then transferred to DSTs for storage. The waste includes the following: 
- Spent cleaning solutions from decontamination and ion exchange regeneration at the 100 Area

- Waste from decontaminating and decommissioning tools and equipment

- Laboratory waste from the 200 Areas and 300 Area

- Fuels fabrication waste from the 300 Area

- Miscellaneous waste from the FFTF operations in the 400 Area.

Additional detail can be found in the DST Part B Permit Application (RL 1991a).

3.1.1.2 Generation. The DSTs do not simply store waste; the tanks are a waste-handling system. The inflows to the DST system include supernate and interstitial liquids pumped from SSTs, laboratory wastes, dilute wastes from across the Hanford Site, and waste from inactive facilities. Outflows include waste destined for evaporation and future pretreatment and vitrification processes. Evaporation decreases the DST waste volume; pretreatment and vitrification remove DST waste and prepare it for disposal.

Projected DST waste generation through 2001 is shown in Figure 3-1 in terms of tank space used versus space available. The average generation rate for DST waste is about 10,400 cubic meters per year before evaporation. This generation rate is based on waste generation projections through 2001 (Table 2-1).

Any TRU solid waste from DST operations that is to be sent to WIPP for disposal will have to comply with WIPP packaging content requirements and TRUPAC II shipping requirements. Current planning calls for all shipments to WIPP to be managed through the WRAP 1 facility or the proposed commercial replacement for the WRAP 2B facility. The potential quantities and future packaging/shipping requirements of such waste are still being studied and are unknown at this time.

\subsubsection{Characterization}

The waste in DSTs consist of solids and liquids. Typically the solids fraction has settled out as a sludge layer. The waste is LLW, TRU waste, and HLW, and is designated based on the waste streams that have been received. The waste in the DST system may not display many of the characteristics listed on the Part A Form 3 Permit Application.

This section summarizes process knowledge and sample analysis for the contents of the DSTs. The assumed waste designations and their bases are described, and schedules for further analysis are given. 
3.1.2.1 Process Knowledge. Several processes contribute to DST waste, as described in Section 3.1.1.1. Waste management practices, including evaporation of tank contents, and transferring waste from tank to tank have intermingled the various types of waste. This intermingling precludes a detailed, quantified characterization of the tank contents based strictly on process knowledge. Instead, the DST waste is described qualitatively based on generation data and sample analysis.

Stratification and segregation have occurred in the tanks as solids have settled out. The consistency of the waste ranges from liquid supernatant to a thick sludge to crusts formed as a top layer.

The major constituents of DST waste are water and sodium salts of aluminate, nitrate, nitrite, phosphate, hydroxide, carbonate, and sulfate. Some calcium and potassium salts also are present. Complexed waste in the DSTs contains sodium salts of the chelating agents ethylenediamine-tetraacetic acid and n-hydroxyethylethylenediamine-tetraacetic acid. There also may be detectable concentrations of halogenated and nonhalogenated organic compounds and heavy metals such as lead, chromium, and cadmium.

In addition, DST waste may be categorized into several types, each having a specific history and character. These wastes types include:

- Double-Shell Slurry/Double-Shell Slurry Feed (DSS/DSSF)

- Neutralized Current Acid Waste

- Neutralized Cladding Removal Solids Waste

- Plutonium Finishing Plant Waste

- Complexant Concentrate Waste

- Dilute Non-complexed Waste

- Concentrated Phosphate Waste.

3.1.2.1.1 Definition of Double-Shell Slurry Feed and Double-Shell Slurry Waste. Double-shell slurry feed is generated by concentrating the dilute waste streams generated by the operating plants to conserve storage space. Double-shell slurry is generated by further concentrating DSSF.

Double-shell slurry feed and DSS are concentrated waste types generated by the evaporation of dilute noncomplexed waste streams to conserve tank space. The DSSF waste has been evaporated up to, but not beyond, the sodium aluminate phase boundary; therefore, it contains no aluminate solids. Double-shell slurry is a more concentrated waste form that is produced by evaporating DSSF past the aluminate boundary. Double-shell slurry contains 
aluminate solids and has a much higher viscosity, which makes retrieval from tanks more difficult and costly.

There are currently 3,607 cubic meters of DSS and 15,702 cubic meters of DSSF.

3.1.2.1.2 Definition of Neutralized Current Acid Waste. The NCAW is also known as PUREX aging waste. The NCAW comprises water, aluminum hydroxide, sodium nitrate, sodium hydroxide, sodium fluoride, cadmium nitrate, sodium nitrite corrosion inhibitors, and the majority of radionuclides from the $\mathrm{N}$ Reactor fuel. Before 1989 process samples analyzed in the laboratory were recycled back to the process system. This practice may have allowed some chemicals added to the samples to enter the aging waste. However, sample analysis has never confirmed the presence of these chemicals in the aging waste. Currently 6,799 cubic meters of NCAW are being stored in DSTs 241-AZ-101 and 102

3.1.2.1.3 Definition of Neutralized Cladding Removal Solids Waste. Cladding removal waste results from dissolving the zircaloy cladding of irradiated nuclear fuel from $N$ Reactor. Neutralizing the waste precipitates most of the zirconium and creates a slurry. The resulting stream is called neutralized cladding removal waste (NCRW).

3.1.2.1.4 Definition and Treatment of Plutonium Finishing Plant Waste. The PFP waste originated from the conversion of plutonium nitrate to oxide or metal and includes TRU laboratory waste and high-salt solvent extraction waste. Current inventory in storage is estimated at 390 to 503 cubic meters. This is stored in Tank 241-SY-102 where it is blended with other 200 West Area wastes.

3.1.2.1.5 Definition and Treatment of Complexant Concentrate Waste. The complexant concentrate results from the concentration of waste containing large amounts of organic complexing agents. The organic complexing compounds were introduced to the waste during strontium recovery at B Plant. No future generation of this waste is planned.

3.1.2.2 Sample Analyses. Samples of the DSTs have been analyzed using EPA SW-846 methodology (EPA 1986). Because no one DST constitutes a "representative" tank, the analytical data from these samples are presented in Table 3-1 as a total mass in all tanks for various chemicals.

3.1.2.3 Waste Designation and Basis. All waste stored in DSTs is designated corrosive dangerous waste (D002) because it has been treated with sodium hydroxide to raise the $\mathrm{pH}$ above 12.5 in preparation for tank storage.

The DST waste typically does not exhibit the characteristic of ignitability (D001). The DST system has received ignitible waste in the past, and the presence of nitrate and nitrite salts in the DST waste may affect this designation. In accordance with Tri-Party Agreement Milestone M-44-00, the data quality objectives process will be used to establish the necessary sampling and analyses for designation, as well as to establish if all applicable treatment standards for waste are being met. The process also will determine which underlying hazardous constituents 
| must be quantified to determine compliance (in accordance with 58 FR 29860 and 59 FR 47982) based on knowledge supplied by Hanford Site waste generators at the original point of generation. The DST waste also is suspected to contain spent solvents including 1,1,1-trichloroethane, methylene chloride, methyl ethyl ketone, hexone, acetone, and cresols (waste codes F001 through F005 are assigned). The DSTs contain waste that meets TCLP criteria for heavy metals contamination: arsenic (D004), barium (D005), cadmium (D006), chromium (D007), lead (D008), mercury (D009), selenium (D010), and silver (D011) although the DST waste may not display the characteristic. All other waste codes on the DST Part A Form 3 Permit Application have also been received for storage.

Radioactive constituents include americium-241, carbon-14, cesium-137, cobalt-60, curium-244, iodine-129, neptunium-237, plutonium-239 and -240, ruthenium- and rhodium-106, selenium-79, strontium-90, technetium-99, and tritium.

3.1.2.4 Uncertainty of Waste Designation. The waste codes previously assigned to DSTs were based on historical knowledge of waste received into the DST system. Additional waste codes may be added or deleted based on the ongoing characterization program. The codes are meant to be all encompassing for the DST system. Waste within specific tanks may be designated using fewer than all of the codes on the list when historical transfer information for the tanks support this option. Otherwise, the DST system will be coded with all hazardous waste codes until the waste is treated to meet $L D R$ requirements.

3.1.2.5 Schedule for Further Characterization. Sampling and analysis of the DST contents is under way and will continue based on prioritization through the Systems Engineering approach.

Sampling is carried out based on the type of waste in each tank. The types of sampling efforts that support various TWRS activities include push-mode and rotary-mode core sampling, grab sampling (bottle-on-a-string), auger sampling, and various types of vapor sampling. Whenever possible, the analytical procedures used by the two onsite laboratories to characterize the DST waste samples are based on methods and techniques found in the EPA Test Methods and Evaluation of Solid Waste (SW-846) (EPA 1986). However, some of these procedures have been modified in terms of sample sizes and preparation techniques to reflect the radioactive nature of the waste samples and the complex constituent matrix. A comprehensive list of the chemical analyses, radionuclides, and physical measurements to be included in the DST characterization effort can be found in the Tank Waste Analysis Plan (WHC 1995c). 


\subsubsection{Storage}

This section describes DST system storage and assesses its compliance with existing regulations.

3.1.3.1 Storage Unit and Capacity. There are 28 DSTs, each with a 4,300-cubic-meter capacity. The 28 tanks are located in 6 tank farms in the 200 Areas of the Hanford Site. Four DSTs in two tank farms are equipped to manage PUREX aging waste. Currently only the two tanks in Tank Farm 241-AZ contain aging waste.

3.1.3.2 Amount in Storage. As of December 31, 1996, the tanks held 72,263 cubic meters of waste (PHMC 1997b). This includes 6,799 cubic meters of PUREX aging waste contained in the two DSTs. Projections indicate that the DSTs could be filled to capacity in 1998 based on current expected generation rates. Consolidation and evaporation of waste in the DSTs will make more of the existing space available for reuse, which will enable the DSTs to receive waste beyond 1998. Tri-Party Agreement Milestone M-42-00 has been renegotiated to postpone the construction of new DSTs indefinitely.

3.1.3.3 Storage Compliance Assessment. The DSTs were reviewed for compliance with interim-status dangerous waste regulations in accordance with Tri-Party Agreement (Ecology et al. 1992) Milestone M-21-00. The assessment for compliance with interim-status regulations noted the following areas of noncompliance:

- Inspection plan

- Waste analysis plan

- Waste characterization

- Training plan.

Compliance action schedules for DSTs are being negotiated in the Tri-Party Agreement. Interim-status compliance for the items listed is completed. Additional DST actions may be required. These actions may include the following:

- Record-keeping system modifications

- Provision of secondary containment for ancillary equipment

- Development of additional leak detection systems

- Development of a closure plan.

\subsubsection{Treatment}

This section discusses current and proposed treatment of DST waste.

3.1.4.1 Current Treatment. The 242-A Evaporator reduces the DST waste volume by evaporative concentration (Chapter 3.0, Section 3.3.1). It began operating in 1977 and has 
evaporated more than 270,000 cubic meters of water from the DST stored waste. The evaporator was restarted for further treatment campaigns in 1994.

3.1.4.2 Proposed Treatment. In addition to those wastes currently being generated for DST storage (supernate and interstitial liquids pumped from SSTs, laboratory wastes, and waste from inactive facilities), wastes currently stored in the DSTs will be treated and disposed of using the same processes and facilities recently adopted by the Tri-Party Agreement. The DST waste will be retrieved, pretreated, and solidified for disposal. Pictorial flow diagrams are shown in Figures 2-2 and 3-2.

Pretreatment separates the DST waste into a LAW and HLW/TRU fraction so that the bulk of the radionuclides are in the HLW. The HLW stream will then be treated to further reduce its volume and increase radionuclide loading if necessary. The LAW will have enough radionuclides removed so that it will meet the Nuclear Regulatory Commission's "incidental waste" classification and the DOE's as low as reasonably achievable (ALARA) policy.

Processes requiring limited development will be used to the extent practical to accomplish the pretreatment function and reduce the $\mathrm{HLW}$ volume to be vitrified. For the LAW pretreatment, these technologies will focus primarily on removing cesium and strontium from the I waste streams to be treated. For the LAW pretreatment, technologies will focus on producing a stream that will create a low-volume, high-loading glass. Development of enhanced technologies, which are expected to include sludge washing, selective leaching, and blending, will continue to be pursued.

In separate facilities, both the LLW and HLW fractions will be vitrified, a process that will destroy or extract organic and cyanide constituents to below treatment standards, neutralize or deactivate dangerous waste and extremely hazardous waste, and immobilize toxic metals. The LLW fraction will be disposed of near surface on site in a retrievable form. The vitrified HLW stream will be stored on site until the Geologic Repository Program is available to receive the waste for disposal.

DOE is privatizing tank waste treatment in two phases. The first phase will be a Proof of Concept/Commercial Demonstration Phase. In Phase I, DOE will select multiple contractors to design and permit facilities to pretreat waste and vitrify the low-level fraction of approximately 6 to 13 percent of the waste over 5 years. Following sufficient design activities that result in acceptable permit applications, one or more contractors will be selected to construct, operate, and deactivate a facility. Phase II will be the full-scale production phase. Two contractors have been selected to begin Phase I activities. They are Lockheed Martin Advanced Environmental Systems and British Nuclear Fuels Limited.

3.1.4.3 Treatment Alternatives and Accelerated Treatment. Alternative treatments are discussed where applicable in Section 3.1.4.2. In addition, a tank waste technical options report has been issued (WHC 1992c) that presents a number of altematives for remediating DSTs. Alternative pretreatment technologies are discussed further in WHC (1993a). 
Treatment of DST waste is on a schedule based primarily on Tri-Party Agreement Milestones M-50-00 (pretreatment), M-60-00 (LLW vitrification), and M-51-00 (HLW vitrification). (Refer to Figure 2-1 for details.) Because of budget limitations, accelerating treatment beyond these milestone dates is not realistic.

\subsubsection{Waste Reduction}

Currently 11 major plants or programs generate DST waste. Annual waste generation for I FYs 1990 through 1996 is listed in Table 3-2. Total waste generation was reduced by 60 percent from 1990 to 1991,54 percent from 1991 to 1992,26 percent from 1992 to 1993 , and 13 percent from 1995 to 1996 . Total waste generation increased by 7 percent between 1993 and 1994 because of SST to DST pumping. Total waste generation increased by 97 percent between 1994 and 1995 because of increased facility terminal cleanout activities and SST to DST pumping. "SST to DST Pumping" refers to pumping liquid waste from SSTs to meet Tri-Party Agreement Milestone M-41-00 requiring all SSTs to be stabilized by the end of FY 2000. Waste reduction activities (current and planned) are outlined for each unit in the Anmual Report of Tank Waste Treatability (WHC 1993d). The four activities include minimizing flush volumes and frequency, pretreating waste (e.g., destroying ammonia), modifying processes, and recycling streams.

Dilute waste received at the DSTs is concentrated by the 242-A Evaporator, further reducing the waste volume by 30 to 95 percent. In 1996, one evaporator campaign was completed. DST waste volume was reduced by 4,200 cubic meters. In an average year, the volume of newly received dilute waste is projected to be reduced by approximately 71 percent.

\section{| 3.1.6 Variances, Equivalency Treatment Petitions, Time Extensions}

The DST waste consists of waste managed as HLW containing dangerous waste constituents. The DST waste is restricted from land disposal because it contains solvent waste (40 CFR 268.30), California List waste (40 CFR 268.32), and waste covered by the Third-Third Promulgation (55 FR 22520). The DST system received corrosive and reactive characteristic waste, TCLP metals and organics, and Washington State-only waste.

The Tri-Party Agreement provides for continued storage of restricted waste until treatment capacity is developed for these wastes. The agreement requires treatment and disposal capacity for these wastes to be developed on the following schedule:

- Low-Level Waste-Disposal of treated waste by vitrification as soon as sufficient quantities are available to facilitate proper treatment and disposal, in accordance with the schedule defined in the Tri-Party Agreement that requires all LLW contained in DSTs and SSTs to be vitrified by 2028 .

- Transuranic Waste - Treatment schedules for TRU waste stored in the DSTs (and SSTs) coincide with those for the treatment of $\mathrm{HLW}$, discussed below. 
- High-Level Waste - Treatment of waste will begin as soon as the HLW vitrification facility has been constructed and sufficient quantities of pretreated waste are available (scheduled for 2009 in accordance with the Tri-Party Agreement). Disposal is intended for a national HLW geologic repository, with an uncertain start-up date.

If additional variances, treatment equivalency petitions, or time extensions are required as a result of delays in the development of treatment, storage, or disposal capacity, they will be applied for in accordance with the procedures detailed in the Tri-Party Agreement or regulations. Variances are expected because of the final sampling and analysis requirements imposed on the final vitrification forms of DST waste.

\section{| 3.2 SINGLE-SHELL TANK WASTE}

The tanks in the SST system are underground, reinforced-concrete, steel-lined tanks used for waste storage. These tanks have held chemically hazardous and radioactive waste generated as a byproduct (according to the Atomic Energy Act of 1954) of processing spent nuclear fuel to recover plutonium, uranium, and neptunium beginning in 1944; additional tanks were constructed as required.

Liquid waste collection and storage in the SST system continued until November 1980. The only material added to the SSTs since 1980 has been water, which was added to tanks 241-C-105 and 241-C-106 to control evaporative cooling. Tank 241-C-106 is still receiving water as necessary. An interim stabilization program was initiated in 1968 to remove pumpable interstitial liquid and supernatant from the SSTs and transfer it to the DSTs. This program primarily is intended to reduce the leak potential of the SSTs.

The SST system consists of 149 tanks containing approximately 136,600 cubic meters of waste. These tanks are located in 12 tank farms with 4 to 18 tanks each in the 200 Areas. The amount of waste contained in the tanks varies from 5 to 95 percent of each tank's capacity and varies in consistency from pumpable liquid to sludge to hard salt cake.

I The SSTs have released an estimated 2,840 cubic meters of liquid to the soil column (Table 3-3). However, after some tanks were declared to be leaking, cooling water may have been added to aid evaporative cooling. It is believed that some of this water did not evaporate and, therefore, went into the ground. As of October 1990, estimates for this additional water release ranged from 190 to 3,000 cubic meters. The past practice was to exclude the cooling water from the leak volume estimate.

In addition, documents show that from 1946 to $1966,456,752$ cubic meters $(120,661,000$ gallons) of liquid wastes were intentionally discharged from SSTs at the Hanford Site directly to the ground on the 200 Areas plateau (WHC 1991c). The majority of this waste was discharged from 1946 to 1958 as a result of the early plutonium and uranium recovery processes conducted in the 221-B Facility (B Plant Complex), 221-T Facility (T Plant Complex), and the 221-U Facility (U Plant). In addition, from 1960 to 1966 laboratory wastes from the 
300 Area and equipment decontamination wastes from the 200 West Area were routed through SSTs before discharge to the ground. No wastes have been discharged intentionally to the

ground from SSTs since 1966. Table 3-3 details the current estimates of releases.

\subsubsection{Generation}

This section describes the waste generation process. Also refer to Section 3.1.1.1 for additional information.

The waste has been generated through a variety of analytical, decladding, and separation processes and associated sitewide operations. The SSTs received this waste from various Hanford Site activities before 1980 .

Waste currently stored in the SSTs was produced by four major chemical processing operations that were conducted from 1944 to 1980 :

- The bismuth phosphate process

- The reduction-oxidation process

- The PUREX process

- The tributyl phosphate process.

The bismuth phosphate, reduction-oxidation, and PUREX Plant processes were specifically designed for plutonium recovery. The initial bismuth phosphate chemical separations process produced large volumes of dilute, low-heat waste. The tributyl phosphate solvent extraction process was designed for the recovery of relatively large amounts of uranium that remained in the bismuth phosphate process waste. The bismuth phosphate process was superseded by the reduction-oxidation process, which was superseded by the PUREX process.

The reduction-oxidation and PUREX processes recovered the uranium and neptunium, as well as the plutonium, from the irradiated reactor fuel. The PUREX process used solvent extraction with tributyl phosphate to separate uranium and plutonium. Chemical removal of the fuel cladding before extraction produced decladding waste with high concentrations of aluminum and zirconium. High-heat-producing isotopes in the waste were separated from the fuel-reprocessing waste by a modified B Plant waste fractionation process. The strontium was separated by an extraction process using complexing agents (e.g., ethylene-diaminetetraacetic acid, n-hydroxyethylethylenediamine-tetraacetic acid, citrate) to prevent transition metal extraction. The cesium was extracted and purified by ion exchange. These isotopes (cesium and strontium) were converted to fluoride and chloride salts and encapsulated in the Waste Encapsulation and Storage Facility, which is part of the B Plant Complex. Sodium hydroxide or sodium carbonate was added to the waste before transfer to the SSTs to create an alkaline solution and to minimize tank corrosion. The processing of irradiated fuels produced waste that included most of the fission products and comparatively small quantities of uranium, plutonium, and other actinides (WHC 1990h). 
Smaller volumes of waste also were added to the SSTs from research and development programs, facility and equipment decontamination, laboratory activities, and the PFP (RL 1995c).

Waste components in the SSTs have settled, stratified, and segregated. The tanks contain a mixture of nonradioactive and radioactive chemicals produced during the various chemical processes. Therefore, determining the actual composition of each tank of waste is a complex process.

Addition of new waste into the SSTs was terminated in November 1980. Water occasionally is added to certain tanks if necessary for evaporative cooling. This water evaporates and does not add to the waste volume.

Any TRU solid waste from SST operations that is to be sent to WIPP for disposal will have to comply with WIPP packaging content requirements and TRUPAC II shipping requirements. Current planning calls for all shipments to WIPP to be managed through the WRAP 1 facility or the commercial replacement for the proposed WRAP 2B facility. The potential quantities and future packaging/shipping requirements of such waste are still being studied and are unknown at this time. For this report, waste generation projections will be incorporated into the receiving facility's projections.

\section{| 3.2.2 Characterization}

The SSTs contain radioactive mixed waste that is solid, liquid, and sludge.

This section discusses the available waste characterization information. Information based on process knowledge and sample analysis is provided along with the waste designations and their bases, the uncertainty related to the designation, and the schedule for further analysis.

| 3.2.2.1 Process Knowledge. The SST system contains irradiated fuel reprocessing waste from separation plants. The tanks received waste from five chemical process activities: the bismuth phosphate, reduction-oxidation, PUREX, and tributyl phosphate processes, and B Plant waste fractionation.

The SSTs contain approximately 136,600 cubic meters of waste as radionuclides and dangerous nonradioactive chemicals. The distribution of the three waste forms (sludge, salt cake, and supernatant) in these tanks is illustrated in Figure 3-3. The salt cake and sludge contain interstitial liquid. The bulk of this liquid, approximately 26,000 cubic meters, is contained in salt cake and is being pumped to the DSTs.

The sludge consists of the solids (hydrous metal oxides, iron, and aluminum) precipitated during the neutralization of acid waste before transfer to the SSTs. Sludges vary greatly in their physical properties. Salt cake contains various salts, primarily sodium nitrate, formed by the evaporation of the water from the waste. Damp salt cake is a jelly-like material; dried salt cake is 
a hard, abrasive, brittle material that may have formed as large single crystals. The salt cake porosity ranges from 10 to 50 percent. The liquid exists as supernate and interstitial fluid (WHC 1990h).

Additional equipment components also are found in the tanks with the process waste. These include metal measuring tapes, level instrumentation, other contaminated scrap, pump heads and shafts, samarium balls, one or more spent fuel elements, and diatamaceous earth. Other nonrecorded items are likely to be contained in the tanks.

| 3.2.2.2 Sample Analyses. Sample analyses are used to evaluate the chemical, physical, and radiological properties of the SST waste and soils that have been contaminated by spills and leaks. This determination will be used to select a disposal alternative that can be executed safely in compliance with RCRA, the State of Washington Hazardous Waste Management Act of 1976, the National Environmental Policy Act of 1969 (NEPA), and the Atomic Energy Act of 1954 regulatory requirements. The waste is extremely varied with respect to radionuclide content and chemical and physical characteristics. This variation among tanks results from the different nuclear fuel processes and the blending, evaporation, and admixture schemes used since 1944.

A remotely operated method for obtaining samples was developed and implemented for sampling the liquid and soft, solid tank waste. One to four core samples were removed from each of 15 SSTs in FY 1985 and 1986 . Core samples were analyzed by the individual segment removed or as a homogenized sample of all segments retrieved from each core. The detailed waste analysis results are reported in Weiss (1986) and Adams et al. (1986).

The SST waste is made up of primarily sodium hydroxide; sodium salts of nitrate, nitrite, carbonate, aluminate, and phosphate; and hydrous oxides of iron and aluminum. A relatively small amount of solvents such as tri-butyl phosphate and normal paraffin hydrocarbon was added to the SST waste during fuel reprocessing, along with water-soluble complexing agents and carboxylic acids from the B Plant waste fractionation process (RL 1995c). Estimates of inventories of nonradioactive chemicals are given in Table 3-1.

Eighteen SSTs were on the Safety Issue Watch List because 140 metric tons of ferrocyanide were placed in the tanks during the 1950 s to precipitate soluble cesium out of solution as cesium-sodium nickel ferrocyanide. If present in sufficient concentration, dry mixtures of ferrocyanide and sodium nitrate may undergo uncontrolled exothermic reactions when heated to temperatures $\left(250^{\circ} \mathrm{C}\right)$ significantly above current tank storage temperatures $\left(<55^{\circ} \mathrm{C}\right)$. Analytical results of recent samples obtained from the ferrocyanide tanks continues to confirm, as do ongoing simulant tests, that ferrocyanide has degraded significantly over the years of storage and ferrocyanide is no longer a major issue. Ferrocyanide concentrations in the worst case tanks have been shown to be less than $1 / 10$ th the amount originally present. Recent information concludes that the cyanides have been destroyed through chemical processes within the tank waste. In 1996 the 18 SSTs were removed from the safety issue list because ferrocyanide concerns were resolved. 
The potential buildup of flammable gases in 19 SSTs and 6 DSTs is another safety issue because a release from the waste could result in concentrations above the lower flammability limit in the tank head space. Work controls were instituted to prevent introduction of spark sources in these tanks, and evaluations were completed to ensure that installed equipment was intrinsically safe. The worst case DST, 241-SY-101, was successfully mitigated in 1994 with the insertion of a mixing pump. The pump is operated up to three times a week to mix the waste and release gases that accumulate in the waste (Babad et al. 1995). Hydrogen monitors have been installed on all 25 flammable gas tanks to monitor for background concentrations and potential releases over time.

A complete, long-term program to characterize SST waste is being conducted by the DOE. This program is detailed in Sasaki (1990). Characterization of all 149 SSTs and 28 DSTs is scheduled to be completed by September 1999 to meet Tri-Party Agreement Milestone M-44-00 (Ecology et al. 1992). The concentration of chemical and radionuclide species of leaked or spilled materials will require future characterization. Characterization results for SSTs are entered into and are available in the integrated database. The regulators have access to this database.

\subsubsection{Waste Designation and Basis. The SSTs are considered to have received ignitable} waste (because of the presence of nitrate), corrosive waste, and TCLP toxic waste. The waste in the SST system is designated identically to the waste in the DST system. Other codes are also applicable (see Table 2-6). These designations are based on process knowledge and limited sample analyses and may change subject to the results of the analysis and characterization of the waste. All of the SST system waste will receive all of the codes from the Part A Form 3 Permit Application. This is because poor information on intra-SST-system transfers and SST-to-DST transfers make distinguishing waste types from the operating records impossible.

| 3.2.2.4 Uncertainty of Waste Designation. The confidence in the current waste code designations is low. The confidence will increase once necessary sampling and analysis work is completed.

\subsubsection{Storage}

This section describes the storage unit, provides the volume currently in storage and projected to be added, and assesses the compliance state of the storage unit.

| 3.2.3.1 Storage Unit and Capacity. Eighty-three of the SSTs are located in the 200 West Area and 66 are in the 200 East Area. The tanks are arranged in 12 tank farms. One hundred thirty-three of the tanks are 22.9 meters in diameter with nominal capacities between 2,000 and 3,800 cubic meters. Sixteen tanks are 6.1 meters in diameter with capacities of 210 cubic meters (WHC 1990c).

| 3.2.3.2 Amount in Storage. The SST waste consists of 136,600 cubic meters of solids including 25,800 cubic meters of interstitial liquid and supernatant. The volume of waste in each tank farm $\mathrm{I}$ is shown in Figure 3-4 (PHMC 1996b). No waste has been added to the tanks since 
November 1980 or will be added in the future. (However, the reference approach for waste retrieval is sluicing, which requires the addition of water or supernate.)

1 3.2.3.3 Storage Compliance Assessment. The SSTs will be closed in accordance with schedules negotiated in the Tri-Party Agreement (Ecology et al. 1996). The SSTs were reviewed for compliance with interim status dangerous waste regulations in accordance with Milestone M-21-00. Compliance action schedules and actions for limited compliance with the interim status requirements during the closure period are being negotiated.

\section{| 3.2.4 Treatment}

This section discusses the current and proposed waste treatment processes.

I 3.2.4.1 Current Treatment. One hundred fifteen of the SSTs have undergone interim stabilization by removal of pumpable liquid. The remaining tanks will undergo interim stabilization operations before disposal as long as the safety class of the tank following liquid removal is acceptable. An interim groundwater monitoring program has been established to comply with the interim-status dangerous waste requirements found in WAC 173-303 and 40 CFR 265.

Sixty of the 22.9-meter-diameter SSTs and 7 of the 6.1-meter-diameter SSTs (WHC 1990c) are assumed to be past leakers. Unique requirements for waste retrieval from these SSTs have not been identified.

3.2.4.2 Proposed and Alternative Treatment. The waste in the SSTs will undergo retrieval and disposal per the latest planning base. Although the selection of the specific alternative will be documented through the NEPA process, the Tri-Party Agreement specifies that SST waste will be treated and disposed of using the DST pretreatment and disposal facilities and that tank 241-C-106 will be the first to undergo retrieval. Closure options, which will identify the level of retrieval necessary, will be documented in a comprehensive tank waste remediation system supplemental environmental impact statement. The supplemental environmental impact statement is in the planning stages.

Waste treated in or retrieved from the SSTs will remain subject to the LDRs unless the following criteria are met.

- Hazardous waste listings applicable to the waste must be identified, and the waste must be delisted in accordance with regulatory requirements

- The treated waste must not exhibit a hazardous waste characteristic (corrosivity, ignitability, reactivity, or TCLP toxicity)

- Treated waste must meet the other treatment standards specified by 40 CFR 268 
Waste that meets these requirements will meet the requirements of the state RCRA program in WAC 173-303-140.

A Tank Waste Technical Options Report was completed in 1992 (WHC 1992c) that presents a number of alternatives for remediating the SSTs and DSTs at the Hanford Site.

| 3.2.4.3 Accelerated Treatment. The SST waste treatment schedule is based primarily on TriParty Agreement Milestones M-41-00 (interim stabilization and M-45-00 (retrieval technology and closure). (Refer to Figure 2-1 for details.) Budget limitations make accelerating treatment beyond these milestone dates unrealistic.

\section{| 3.2.5 Waste Reduction}

A waste evaporation program was initiated in 1965 to reduce the volume of liquid waste that potentially could leak and contaminate the soil surrounding the tanks. The supernatant liquids were extracted from the SSTs, evaporated to a slurry, and replaced in the tanks for storage. In 1974 two evaporators were installed and used to evaporate water. Further efforts to reduce the potential for leakage include the transfer of waste materials from the SSTs to DSTs.

During 1996, portions of tanks T-111, T-104, S-108, S-110, and BY-109 were transferred to DSTs.

\subsubsection{Variances, Treatment Equivalency Petitions, Time Extensions}

The SST waste consists of radioactive waste mixed with dangerous waste constituents.

I The Tri-Party Agreement (Ecology et al. 1989) provides for development of treatment and disposal units for the SST waste as follows:

- Complete SST interim stabilization by September 2000

- Develop SST waste retrieval technology and complete scale-model testing by September 1994

- Initiate full-scale tank demonstration of SST waste retrieval technology by October 1997

- Initiate tank waste retrieval from one SST by December 2003

- Complete closure of all 149 SSTs by September 2024.

If variances, treatment equivalency petitions, or time extensions are required as a result of delays in the development of treatment, storage, or disposal capacity, they will be applied for in accordance with the procedures detailed in the Tri-Party Agreement or regulations. 


\section{| 3.3 242-A EVAPORATOR PROCESS CONDENSATE}

The 242-A Evaporator concentrates the low-level liquid waste that is stored in underground DSTs. The DSTs store low-heat-generating waste that contains relatively small amounts of fission products.

The 242-A Evaporator concentrates liquid waste by evaporation. This process reduces the tank waste volume and, hence, the number of DSTs required for storage. The 242-A Evaporator started operating in September 1977; ongoing upgrades will extend its useful life.

Before 1989, the process condensate was routed to retention basins, analyzed for radionuclides and ammonia, and discharged to a crib. In April 1989, process knowledge on listed waste management applicability became available and high concentrations of ammonia were detected in the process condensate and discharge to the crib was discontinued. The 242-A Evaporator restarted in calendar year 1994. The process condensate is currently stored in the LERF and treated in the 200 Area Effluent Treatment Facility.

The ETF also will treat other waste water streams that are generated from Hanford Site waste management and remediation activities. This waste water will include both mixed waste and non-hazardous waste. Some of this waste water will be stored in the LERF before treatment in the ETF.

\section{| 3.3.1 Generation}

The 242-A Evaporator concentrates liquid LLW by evaporation. The 242-A Evaporator receives a mixture of waste from DST evaporator feed tanks. These tanks receive dilute wastes from other DSTs after the waste has been characterized to determine the suitability of the waste for evaporation. A simplified schematic of 242-A Evaporator process operations is shown in Figure 3-5.

The 242-A Evaporator heats the feed at reduced pressure and evaporates off some of the water and volatile organic constituents from the slurry. The vapor fraction and slurry fraction are then processed separately. The vapor fraction is condensed, filtered, and discharged to the LERF as process condensate. The remaining slurry is recirculated. When the slurry is sufficiently concentrated, it is pumped to underground storage in DSTs.

The 242-A Evaporator has generated 40,000 cubic meters of process condensate since its restart in 1994. One evaporator campaign in May 1996 generated about 4,800 cubic meters of process condensate. Approximately two evaporator campaigns are planned per year; each campaign is expected to generate about 3,800 cubic meters ( 1 million gallons) of process condensate. 
Initially, process condensate from the 242-A Evaporator was the only mixed waste identified for storage and treatment in the LERF and ETF. However, the ETF was designed to treat a variety of radioactive and hazardous liquid waste generated at the Hanford Site. As waste management and remediation activities have progressed, waste water from various remediation and waste management activities also have been identified for treatment and storage in the ETF and LERF.

The process condensate is a dangerous waste because it is derived from DST waste. Other waste water streams that will be managed at the ETF and LERF are generated from waste management and remediation activities at the Hanford Site. These streams include, but are not limited to, contaminated groundwater from pump-and-treat operations, water from the deactivation activities such as water from the spent fuel storage basins at deactivated reactors, laboratory waste water from unused samples and sample analyses, and leachate from landfills.

\subsubsection{Characterization}

Process condensate has been designated a dangerous waste because it is derived from a waste that may contain the spent halogenated and nonhalogenated solvents 1,1,1-trichloroethane, methylene chloride, acetone, methyl isobutyl ketone, cresols, and methyl ethyl ketone. These are the constituents under waste codes F001 and F005.

Because the constituents in the individual waste water streams that will be treated or stored in the LERF vary, each stream will be characterized and evaluated for acceptability on a case-bycase basis.

\subsubsection{Uncertainty of Waste Designation. The current designations are considered accurate}

3.3.2.2 Schedule for Further Characterization. The treated effluent from the ETF is characterized to confirm that it has been properly treated and meets discharge and delisting limits. When the treated effluent is shown to meet these limits, it is considered delisted and no longer a hazardous waste.

\section{| 3.3.3 Storage}

The 242-A Evaporator was modernized and restarted in 1994. The process condensate from the evaporator will be stored and treated at the LERF until treated in the 200 Area ETF. The LERF can hold about 49,000 cubic meters of liquid effluents. A schematic of the LERF is shown in Figure 3-6.

The LERF consists of surface impoundments that comply with interim-status design and operating requirements. A Part B permit application was prepared and submitted in accordance with Tri-Party Agreement (Ecology et al. 1989) Milestone M-20-47 detailing the compliance of the LERF to RCRA final-status design and operating requirements. At RL's request, in 
December 1994, the EPA issued a letter stating that the treatment proposed was consistent with the treatment described in $40 \mathrm{CFR}$ 268.4. The LERF equilizes the flow and $\mathrm{pH}$ of the feed to the ETF to ensure consistent treatment and minimize process upsets. This has allowed the continued use of LERF for treating and storing LDR waste. The Tri-Party Agreement milestones to discontinue process condensate discharge to LERF (M-26-03) and to remove residues from LERF (M-26-04) no longer apply. These milestones have been deleted by Change Request M-26-95-01, approved June 28, 1995.

\section{| 3.3.4 Treatment}

I 3.3.4.1 Planned Treatment. The ETF treats aqueous wastes, including process condensate, and prepares the waste for disposal. The ETF includes the following treatment processes.

- Adjustment of $\mathrm{pH}$ is done in several steps.

- Remove suspended particles with a roughing filter.

- Convert organic compounds into carbon dioxide and water and destroy cyanides in an organic destruction unit using hydrogen peroxide and ultraviolet light.

- Filter out residual particulates using a fine filter.

- Remove the carbon dioxide generated during treatment using degasification.

- Use reverse osmosis to remove dissolved aqueous salts (including metal ions, radionuclides, and ammonium sulfate), producing a secondary waste stream that will be further concentrated by evaporation.

- Treat the stream by ion exchange to remove residual dissolved aqueous salts not removed by reverse osmosis.

- Neutralize the treated stream as necessary and send to verification tanks. A system of three verification tanks holds the treated effluent for sampling before discharge to a state-approved land disposal structure. A recycle loop is provided in case verification analyses show that rework is required to meet permit conditions.

- Send secondary waste (primarily produced from the reverse osmosis step, regeneration wastes from the ion exchange step, and blow-down from the two filtration steps) to an evaporation process consisting of a mechanical vapor recompression evaporator and a thin-film dryer. Feed to the evaporation process will be routinely analyzed to determine the nature of the dry secondary waste product. If the dried secondary waste product is a hazardous or dangerous waste, it will be sent to the appropriate hazardous waste disposal facility. If it is not hazardous or dangerous, the drums will be disposed of at the low-level burial grounds. 
3.3.4.2 Treatment Alternatives and Accelerated Treatment. The treatment method for 242-A process condensate and other aqueous wastes began with ETF startup in November 1995.

\section{3.3.5 Waste Reduction}

1 Planned treatment of the process condensate and other waste water streams will result in a nondangerous liquid stream acceptable for discharge to the ground and a solid waste form acceptable for storage at the CWC and/or disposal on the Hanford Site.

In 1995 the 242-A Evaporator was modified to reduce the introduction of filtered raw water by recycling a portion of the process condensate stream through the pump seal water system and the de-entrainment pad sprayers. This modification reduced the amount of condensate sent to the LERF by 400 cubic meters in 1995 and 200 cubic meters in 1996.

Treating 37,500 cubic meters of process condensate in 1996 produced 69 cubic meters (331 drums) of dry secondary waste. The secondary waste was designated as low-level mixed waste.

\subsubsection{Variances, Treatment Equivalency Petitions, Time Extensions}

The 242-A Evaporator process condensate is a LLW mixed waste that is LDR because it is derived from DST wastes that received spent solvents. Process condensate generated is discharged to and stored in the LERF until it is treated at the ETF.

In June 1995, the EPA granted a petition (60 FR 31115) submitted to $R L$ for the delisting of effluent treated at the ETF as hazardous waste. Part B Permit applications have been submitted for the 242-A Evaporator (completed June 1991), the LERF (completed June 1991), and the ETF (completed August 1993). New revisions of these permits applications are being drafted for submittal to Ecology in 1997.

No additional variances, treatment equivalence petitions, or time extensions are expected, unless the delisting petition requires expansion to delist wastes not included within the existing scope.

\subsection{PUREX STORAGE TUNNELS WASTE}

The PUREX Storage Tunnels 1 and 2 both contain elemental lead. The PUREX Storage Tunnel 2 contains silver (mostly as silver nitrate), elemental mercury, cadmium, and chromium. The lead is in jumper counterweights and equipment weights and shielding. The silver is in discarded silver reactors. The mercury is sealed inside thermowells that are an integral part of the irradiated fuel dissolvers. The cadmium is present as elemental cadmium attached to equipment 
for neutron shielding. The chromium is a corrosion byproduct from a failed stainless steel process concentrator.

In June 1996, barium, absorbed mineral oil, and additional amounts of cadmium, chromium, and lead were added to the PUREX Storage Tunnel 2 from the 324 and 325 Buildings. Barium is present as dispersible particulate contaminated with process materials from the 324 Building process. Cadmium is present as dispersible particulate and dried melter feed residue removed from process equipment. Chromium is present as dispersible particulate and dried melter feed residue. Lead is present as dispersible particulate, dried melter feed residue, and liquid metal seal material. The mineral oil is contained within an absorbent material.

As Hanford facilities are deactivated other equipment and materials containing these and other waste types may be added to the tunnels.

\subsubsection{Generation}

Elemental lead waste was generated in the PUREX process as an integral part of equipment, such as process pipe jumpers, jumper alignment tools, and shielding equipment. Historically, elemental lead was used as weights, counterweights, and radiation shielding in the fabrication of process equipment used in the PUREX Plant; generally, the lead was encased in steel (carbon or stainless) to facilitate its attachment to process equipment. Counterweights were used to facilitate remote installation of in-cell process and service piping (jumpers). A jumper alignment tool may have contained as much as 680 kilograms of lead. This tool was used as a weight to pull down the free end of a jumper so the connecting parts aligned vertically and the connection could be made.

I Silver in the form of silver salts deposited on unglazed ceramic packing was contained within the discarded silver reactors stored in Tunnel 2 . Three silver reactors were used to remove radioactive iodine from the offgas streams of the irradiated reactor fuel dissolvers in the PUREX process. The silver reactor vessel contained two beds of packing. The packing was coated initially with 114 kilograms of silver nitrate used for iodine retention. Nozzles on the top of the reactor were provided to allow flushing and/or regeneration of the packing with silver nitrate solution as the need arose.

Experience showed that after extended use, the silver reactors lost efficiency. This loss in efficiency normally occurred when about one-half the silver nitrate on the packing had been converted to silver iodide. Other competing reactions such as reduction of silver nitrate to metallic silver and formation of silver chloride also occurred and affected silver reactor efficiency. Therefore, the silver reactor was regenerated with fresh silver nitrate periodically. Thus, the packing of the discarded silver reactor contained a mixture of silver nitrate, silver halides, and silver fines. 
Elemental mercury waste was generated when dissolvers in the PUREX process failed or were deemed to be obsolete (discarded). The mercury became a waste because its removal from the discarded dissolver was not practical.

The elemental mercury was sealed inside thermowells, which were an integral part of reactor fuel dissolvers used at the PUREX. Plant. Each dissolver had two thermowells. Each thermowell consisted of a 2.9-meter length of stainless steel pipe with an extension welded to the downside end. The lower end butted against the outer surface of the internal slotted bar screen that separates the undissolved fuel elements from the outer solution chamber of the annular I dissolver. The mercury served to transfer heat from the dissolver interior to the temperature sensor mounted within the thermowell. This mercury remains in the thermowells of discarded dissolvers. In preparation for storage, the thermowell was sealed with a stainless steel nozzle plug. In storage, the discarded dissolver rests in an inclined position in a cradle on a rail car. Secondary containment is provided by the dissolver vessel itself.

I As of December 1996, three dissolvers have been discarded, one in 1971, a second in 1972, and a third in 1986. The first two dissolvers each contain 45 kilograms of elemental mercury; the third one contains 38 kilograms. All three dissolvers are stored on rail cars in PUREX Storage Tunnel 2 (RL 1990b).

Cadmium may be present in the PUREX Storage Tunnel 2 as elemental cadmium attached to equipment for neutron shielding. The presence of cadmium is determined on the basis of process knowledge and the design of equipment used during PUREX operation known to possibly contain cadmium metal.

Chromium is present in the PUREX Storage Tunnel 2 as a corrosion byproduct of the stainless steel from a failed process concentrator. This concentrator was evaluated for reuse in 1986 and was determined to be unacceptable because of incompatibilities and a potential short service life. The concentrator was inspected and found to contain silicate solids that contained high levels of chromium.

In the 324 Building waste, lead is present in dispersible debris, dried melter feed residue, and liquid metal seal material from the 324 Building process. The dispersible debris waste consists of dirt, dust, process residues, equipment, and tools collected from the 324 Building $\mathrm{B}$ Cell floor. This debris does not contain any hazardous components, however it has been contaminated with process feed solutions that contained heavy metals. The residual dried melter feed was removed from process equipment after water evaporation from the melter feed slurry used in the Federal Republic of Germany repository program. The liquid metal seal, used as a seal material in a glass melter, is inherently hazardous. Barium also is present in the dispersible debris. I Cadmium is present in the dispersible debris and liquid metal seal and chromium are present in the dispersible debris and residual dried melter feed. Mineral oil in an absorbent material is also present.

During the PUREX surveillance and maintenance phase, waste from Hanford facilities may be stored in the PUREX tunnels. 


\subsubsection{Characterization}

This section discusses the available waste characterization information. Information based on process knowledge and sample analyses is provided along with the waste designations and their bases, the uncertainty of the designations, and the schedule for further analysis.

| 3.4.2.1 Process Knowledge. The quantity of lead generated was identified from a review of fabrication and design drawings for each piece of equipment placed in storage if the lead weight,

I counterweight, or shielding was specifically detailed. The silver salts quantity was estimated from the knowledge of the amount of silver nitrate placed on the bedding and the regeneration history of the silver reactors. For accountability purposes, the total silver content was considered to be silver nitrate, the salt that exhibits the characteristics of both ignitability and TCLP toxicity.

I Characterization of the mercury waste relied on fabrication and installation specifications. I The quantity of mercury present in each dissolver was documented on the fabrication drawings. None of the mercury will evaporate because each thermowell is sealed.

The quantity of cadmium was estimated from the dimensions of the cadmium metal sheets attached to the equipment. The quantity of chromium was estimated from knowledge of silicate solids that have high levels of chromium and are contained within the failed concentrator sampled during PUREX operation. The quantity of barium, lead, cadmium, mineral oil, and chromium from the 324 Building waste was estimated from process knowledge.

3.4.2.2 Sample Analyses. Sampling and chemical analysis were not performed on waste associated with the radioactive discarded equipment placed in the PUREX Storage Tunnels. The quantity of waste in storage was determined from process knowledge and equipment design. Provisions for taking samples of the bedding were not provided in the design of the silver reactor | vessels. Therefore, sampling and chemical analysis were not performed for silver salts before placing a silver reactor in storage.

I Sampling and chemical analysis was not performed on mercury associated with the dissolvers. The need for sample analyses will be evaluated during planning for closure of the PUREX Plant, including the storage tunnels. A PUREX preclosure work plan was submitted to I Ecology and the EPA in July 1996.

| 3.4.2.3 Waste Designation and Basis. Elemental lead exhibits the characteristic of toxicity as determined by the TCLP and is designated D008. The form of lead present could produce an extract greater that 500 milligrams per liter should it be exposed to a leachate. However, because the bulk of the lead is encased in steel on rail cars that isolate the lead from other materials stored within the tunnel, the potential for exposure of lead to a leachate is considered to be negligible.

Silver salts exhibit the characteristics of toxicity as determined by the TCLP and are designated D011 as well as D001 because of their oxidizer characteristics. The form of silver present could produce an extract having greater than 500 milligrams of silver per liter should the 
salts be exposed to a leachate. Although nitrate is an oxidizer and is designated D001 (ignitable), the dispersion of a nitrate salt on unglazed ceramic packing contained within a stainless steel vessel and isolated from other materials stored within the storage tunnel results in a probability for ignition to be considered negligible.

The designation of mercury waste is based on process knowledge and the fabrication and installation specifications. Elemental mercury exhibits the characteristic of toxicity as determined by the TCLP and is designated D009. The quantity of mercury present, if exposed to a leachate, could produce an extract greater than 0.2 milligram per liter.

Cadmium exhibits the characteristics of toxicity as determined by the TCLP and is designated D006. The form of cadmium present could produce an extract greater than 100 milligrams per liter should it be exposed to a leachate.

Chromium exhibits the characteristics of toxicity as determined by the TCLP and is designated D007. If exposed to a leachate, the quantity of chromium present could produce an extract of greater than 5 milligrams per liter.

Barium exhibits the characteristics of toxicity as determined by the TCLP and is designated D005. The form of barium present could produce an extract of more than 100 milligrams per liter if it were exposed to a leachate.

Mineral oil is state-only toxic, per information on the manufacturer's material safety data sheet.

3.4.2.4 Uncertainty of Waste Designation. The designated waste codes for the PUREX tunnel waste are considered accurate.

3.4.2.5 Schedule for Further Characterization. No schedule for further characterization has been established. The need for additional waste characterization will be evaluated during planning for closing the PUREX Storage Tunnels.

\subsubsection{Storage}

This section discusses the PUREX Storage Tunnels, provides their storage capacity and the amount of waste stored, and assesses the compliance status of the storage unit.

3.4.3.1 Storage Unit Capacity. The PUREX Storage Tunnels are a mixed waste storage unit. The two tunnels are connected to the PUREX Plant and, combined, provide storage space for 48 rail cars. The PUREX Storage Tunnels provide long-term storage for process equipment removed from the PUREX Plant and other onsite sources. Equipment transfers into the PUREX Storage Tunnels are made as needed. Radioactively contaminated equipment is loaded on rail cars and remotely transferred into the PUREX Storage Tunnels. Rail cars act as both transport and a storage platform for equipment placed in the tunnels. 
DOE/RL-97-14

The tunnels are weather-tight structures covered by 2.4 meters of earth. This design serves to protect the stored equipment from exposure to natural elements, provides external radiation shielding from the radioactive equipment stored in the tunnels, and protects the environment.

Tunnel 1 (218-E-14) was completed in 1956 as part of the PUREX Plant construction project and provides storage for eight rail cars. Tunnel 1 was filled to capacity (approximately 600 cubic meters of waste) in 1965 and subsequently was secured. No elemental mercury waste is stored in Tunnel 1.

Tunnel 2 (218-E-15) was an expansion project constructed in 1964. This tunnel is designed differently from and is considerably longer than Tunnel 1, providing storage space for 40 rail cars. | Each rail car can hold 497 cubic meters of waste. To date, 28 rail cars containing 2,204 cubic meters of discarded equipment and associated waste have been placed in the tunnel, filling

170 percent of the storage area. Sufficient storage capacity remains for all future waste projected to be generated. A more complete description of the PUREX Storage Tunnels may be found in PUREX Storage Tunnels Dangerous Waste Permit Application, Rev. 2 (RL 1990b).

| 3.4.3.2 Amount in Storage. As of July 1996, 0.89 cubic meter of elemental lead is stored in PUREX Storage Tunnels 1 and 2. PUREX Storage Tunnel 2 currently holds 0.07 cubic meter of silver nitrate, 0.01 cubic meter of elemental mercury, $7.88 \times 10^{-3}$ cubic meter of cadmium, and $1.26 \times 10^{-3}$ cubic meter of chromium, $8.29 \times 10^{-4}$ cubic meter of barium, and $7.4 \times 10^{-3}$ cubic meter of mineral oil. Table 3-4 lists storage tunnel inventories and waste transfer dates.

\section{3.4.4 Treatment}

| 3.4.4.1 Planned Treatment. Planned treatment of the elemental lead and mercury, and the silver salts associated with the process equipment stored in the tunnels is presented in RL (1990b). The elemental lead will be removed, where feasible, from the process equipment to reduce the volume to be treated. The elemental lead and the silver salts located in the silver reactors are planned to be treated by encapsulating the material in a cementitious grout that immobilizes the lead and silver. No planned treatment has been developed for the cadmium, chromium, barium, and selenium associated with the process equipment stored in the tunnels. The EPA-required treatment for elemental mercury is amalgamation. Therefore, the treatment of choice is the current approach of adding zinc powder to create an amalgam. An alternative treatment being considered is to mineralize the elemental mercury (creating mercury sulfide).

3.4.4.2 Treatment Alternatives. Alternatives to this process have not been studied. As necessary, this will be done as part of plant closure.

| 3.4.4.3 Accelerated Treatment. A schedule for treatment of this waste has not been established. Waste from the tunnels will be handled along with the similar materials currently in the PUREX Canyon when PUREX is decontaminated and decommissioned. PUREX decontamination and decommissioning, along with treatment of the Tunnel waste, is contingent on the completion of the Sitewide Land Use Plan, the Sitewide Decontamination and 
Decommissioning Priority Schedule, the Environmental Impact Statement, and public comments. A basis for the treatment plan for the wastes associated with PUREX storage will be developed after all of these items are complete.

\subsubsection{Waste Reduction}

Since early 1987, the use of lead in the design and fabrication of replacement equipment for the PUREX Plant has been discontinued wherever feasible.

\section{| 3.4.6 Variances, Treatment Equivalency Petitions, Time Extensions}

Waste was placed in the PUREX storage tunnels before November 1987 and is, therefore, not subject to LDRs until it is removed from the tunnels. Removal is planned as part of the PUREX Plant closure. At that time the waste will be removed from the PUREX Storage Tunnels, treated to comply with LDR treatment standards, and disposed of at a RCRA-compliant disposal facility.

If variances, treatment equivalency petitions, or time extensions are required because of delays in the development of treatment, storage, or disposal capacity, they will be applied for in accordance with the procedures detailed in the Tri-Party Agreement or regulations.

\section{3.5 PUREX CONTAINMENT BUILDING (LEAD, CHROMIUM, AND CADMIUM)}

The PUREX containment building is permitted for the storage of solid mixed waste containing barium, cadmium, chromium, lead, silver, selenium, and light absorbed mineral oil. Since December 7, 1987, radioactively contaminated process equipment that was removed from the canyon area and contained lead and cadmium has been stored in the hot pipe trench adjacent to D-Cell and inside F-Cell (F17 position). As part of deactivation activities, the solid mixed waste stored on the canyon deck was moved to PUREX Storage Tunnel 2. The mixed waste remaining in F-Cell consists mainly of concrete and tank dunnage corrosion products.

\subsubsection{Generation}

The waste stored in Position F17 in F-Cell consists of concrete debris collected from the floor of E-Cell. The concrete material was retrieved from the floor during the replacement of Tank E3. Approximately 1.0 cubic meter of debris, weighing approximately $4,100 \mathrm{~kg}$, is contained in a $1.2 \times 1.8 \times 0.6$-meter carbon steel scrap hopper. The debris contains regulated quantities of chromium. 


\section{| 3.5.2 Characterization}

This section discusses the waste characterization and its basis. The waste designation, the uncertainty of the designation, and the schedule for further characterization also are provided.

1 3.5.2.1 Process Knowledge. The waste stored in Position F17 in F-Cell consists of concrete debris collected from the floor of E-Cell. The concrete material was retrieved from the floor during the replacement of Tank E3.

3.5.2.2 Sample Analyses. Sample analyses were conducted to characterize the E-Cell floor solids in which the regulated quantities of chromium were detected.

3.5.2.3 Waste Designation and Basis. The waste is designated TCLP toxic for chromium (D007).

3.5.2.4 Uncertainty of Waste Designation. The waste designation is accurately known, based on process knowledge.

| 3.5.2.5 Schedule for Further Characterization. No further characterization of this waste is scheduled.

\subsubsection{Storage}

This section describes the storage unit and assesses its compliance status.

I 3.5.3.1 Description of Storage Unit and Capacity. The PUREX containment building is a portion of the plant with a thick concrete floor, walls, and ceiling (up to 1.8 meters thick). Work in the canyon is generally performed remotely because of high radiation levels.

Because any waste in the containment building is located inside the 202-A Building, the waste is protected from external environmental forces such as wind, rain, and run-on flooding.

| 3.5.3.2 Amount in Storage. The quantity of chromium waste in storage is $1.4 \times 10^{-4}$ cubic meter (1 kg of chromium).

| 3.5.3.3 Storage Compliance Assessment. Containment building storage of mixed waste on the canyon deck of the 202-A Building is addressed in revisions of the Part A Permit application for the PUREX Plant. The PUREX Plant waste management unit was reviewed for compliance with interim-status dangerous waste regulations in accordance with Tri-Party Agreement

| Milestone M-21-00 (Ecology et al. 1989). No interim status compliance deficiencies were noted. 


\section{| 3.5.4 Treatment}

3.5.4.1 Planned and Alternative Treatments. There are currently no plans for treatment of the chromium-contaminated concrete solids from the E Cell floor. Interim storage in F-Cell was chosen as the best stabilization method for this material. The waste in F-Cell will remain in place through the surveillance and maintenance phase and will be dispositioned during closure.

3.5.4.2 Accelerated Treatment. A schedule for treatment of this waste has not been I established. The material stored in the containment building will be addressed as part of PUREX I Plant closure.

\section{| 3.5.5 Waste Reduction}

Additional transfers into the PUREX containment building as part of stabilization or surveillance and maintenance are not expected.

\section{| 3.5.6 Variances, Treatment Equivalency Petitions, Time Extensions}

Removal of the mixed waste remaining in the containment building will be addressed as part of the PUREX Plant closure. At that time, waste will be removed from the PUREX canyon deck, treated to comply with LDR treatment standards, and disposed of at a permitted disposal facility.

If variances, treatment equivalency petitions, or time extensions are required because of delays in the development of treatment, storage, or disposal capacity, they will be applied for in accordance with the procedures detailed in the Tri-Party Agreement or regulations.

\section{| 3.6 CENTRAL WASTE COMPLEX STORED LOW-LEVEL, TRANSURANIC, AND POLYCHLORINATED BIPHENYL WASTE}

The CWC receives radioactive solid waste and provides temporary storage until treatment by DOE facilities or by commercial entities.

Waste is received at the CWC from all radioactive waste generators at the Hanford Site and any offsite generators that are authorized by the DOE to ship waste to the Hanford Site for treatment, storage, and disposal. The waste received at the $\mathrm{CWC}$ is generated by ongoing Site operations (e.g., facility stabilization, waste management) and research and development activities conducted at the site (e.g., SST waste sampling and analysis). Offsite waste has been primarily from DOE research facilities and other DOE sites. The characteristics of the waste received at the CWC vary greatly from waste that is nondangerous LLW to TRU dangerous waste. The CWC currently stores, as of June 30, 1996, approximately 6,906 cubic meters of mixed LLW 
I subject to LDRs. (TRU mixed waste is no longer subject to the LDR prohibition through recent legislation [61 FR 60704].) Other dangerous waste that is not restricted from land disposal is stored at the CWC and is not included in these figures. (Including this non-LDR waste, approximately 8,600 cubic meters of waste are stored.)

The best available generation information is maintained in the computerized Solid Waste Information and Tracking System database. The Solid Waste Information and Tracking System contains only information provided by the waste generator. In the past, exhaustive waste descriptions that could be used to accurately classify a waste were not required, and data entries such as "contaminated debris" and "mixed fission products" were common.

No treatment units currently exist for TRU or LLW contaminated with PCBs. Therefore, this waste is being held in storage at the CWC until treatment capability exists. The Hanford Site PCBs inventory includes contaminated liquids (PCB-contaminated hydraulic fluid), contaminated combustible solids, and contaminated equipment (transformers, capacitors, and fluorescent light ballasts). As of June 30, 1996, 182 cubic meters of PCB-contaminated LLW and 78.4 cubic meters of PCB-contaminated TRU waste are in storage.

\section{| 3.6.1 Generation}

This section describes the generation of RMW and radioactive PCB waste shipped to the CWC.

| 3.6.1.1 Mixed Waste Generation. The majority of waste shipped to the CWC is generated in small quantities by routine plant operation and maintenance activities. Specifying generation rates and types of waste generated by each plant is difficult because this waste is not generated as a direct result of process operations. The overall volumes of mixed waste projected to be generated

| are given in Table 3-5. No data are available on the fraction of this waste that will be subject to LDRs, but the majority of this newly generated mixed waste probably will be subject to the LDRs. The dangerous waste designation of each container of waste is determined at its point of generation based on process knowledge of the waste placed in the container or sample analysis if sufficient process knowledge is unavailable. The major plants that generate land disposal restricted mixed waste and the general type of waste they generate are discussed below.

In the past, the PUREX Plant, located in the 200 East Area, was used to process irradiated nuclear fuel from $\mathrm{N}$ Reactor. The PUREX process used a nitric acid solution to dissolve the fuel and a solvent extraction process to separate the various fission products from the uranium, plutonium, and neptunium product streams. Radioactive solid waste is generated in all parts of the PUREX Plant from routine laboratory operations to equipment maintenance. Typically, the mixed solid waste generated at the PUREX Plant includes lead shielding, decontamination solvents, mercury-filled light tubes, and other nonroutinely generated radioactive solid waste. 
The PFP, located in the 200 West Area, has been used to process plutonium nitrate solutions from the PUREX Plant, plutonium oxide, and plutonium scrap into metal. The plant consists of several facilities, including the Plutonium Reclamation Facility, the Remote Mechanical ' $\mathrm{C}$ ' Line (RMC), and the Product Handling Facility. Several radioactive mixed waste streams including lead, PCBs, and laboratory wastes are routinely generated at the PFP and shipped to the CWC.

The Uranium Oxide Plant, located in the 200 West Area, converted uranyl nitrate solution generated from the reprocessing of $\mathrm{N}$ Reactor fuel to uranium oxide solids that were shipped off site for reuse. The plant is currently shut down awaiting decontamination and decommissioning. The primary sources of mixed waste at the Uranium Oxide Plant are solvents and mineral acids $\left(\mathrm{HNO}_{3}\right.$ and $\mathrm{H}_{2} \mathrm{SO}_{4}$ ) used for decontamination or equipment maintenance in radiation areas. Other sources of LDR mixed waste at the Uranium Oxide Plant include contaminated fluorescent tubes and failed equipment.

The 222-S laboratories, located in the 200 West Area, are used to analyze radioactive samples in support of waste management operations and tank characterization. These operations generate both solid and liquid mixed LLW. The solid waste generated by this laboratory includes radioactively contaminated lead, outdated chemicals and reagents, and equipment and absorbent materials contaminated with radioactive waste. The liquid mixed LLW is generated when using organic solvents to analyze radioisotopes.

B Plant, located in the 200 East Area, was used to separate cesium and strontium from waste streams to be sent to SSTs and DSTs from PUREX. Maintenance activities in B Plant generate small quantities of solid waste, such as lead shielding, equipment decontamination agents, paint and painting supplies, and fluorescent light ballasts. This contact-handled and remote-handled waste is generated as needed because of plant maintenance and upgrading.

T Plant, located in the 200 West Area, is used to decontaminate failed equipment to facilitate its repair, reuse, or disposal. The solid waste generated as a result of these operations includes spent solvents, failed equipment, lead shielding, paint and painting supplies, and metallic vapor lights.

N Reactor, located in the $100 \mathrm{~N}$ Area, is shut down and in deactivated status. There are numerous sources of mixed LLW in the 100-N Area that generate waste oils, solvents, and decontamination solutions that in the past have been determined to be dangerous waste. The 100-H Area was the location of the 183-H Solar Evaporation Basins, which was the source of a large quantity of waste (approximately 3,375 cubic meters).

The 300 Area Fuels Manufacturing Operations generated several mixed LLW streams. These operations have been shut down since December 1986, and the only waste generated is from decontaminating and closing these operations. The waste has been transferred to the $\mathrm{CWC}$, or off site if determined nonradioactive, as part of the closure activities for the 303-K Facility. 
The FFTF, in the 400 Area, and associated research and development activities generate several waste streams that are mixed waste. This waste includes sodium, contaminated lead residual waste, and condensate from a containment building recirculation cooler. Contaminated lead residual waste is generated from the removal of lead shielding for repair and replacement. Sodium waste is generated during the decontamination of refueling equipment or during system maintenance work. Condensate from a recirculation cooler in the containment building sometimes has high enough concentrations of lead and tritium to be regulated as mixed waste.

The research activities conducted by Pacific Northwest National Laboratory (PNNL) in the 300 Area and North Richland generate numerous small-volume mixed waste streams that are land-disposal restricted. The laboratory waste may contain materials that are designated TCLP toxic (D004-D043), ignitable (D001), corrosive (D002), F-listed (F001-F005) or state criteria (WT01, WT02, WP01, WP02, WP03). The waste is generated through various research and development activities including analysis of samples containing toxic metals and solvents (i.e., tank waste samples), the disposal of contaminated equipment, and the use of scintillation cocktails in radionuclide analyses.

The operation and maintenance of the SST and DST tank farms located in the 200 Areas generates several types of mixed waste. The waste includes equipment used for tank sampling and characterization, failed equipment and instrumentation, and small quantities of tank waste absorbed on clothing or rags. These waste streams may be designated by some or all of the waste codes applicable to DSTs. These codes include corrosivity (D002); TCLP toxicity for arsenic (D004), barium (D005), cadmium (D006), chromium (D007), lead (D008), mercury (D009), selenium (D010), and silver (D011); spent halogenated solvents (F001 and F002); spent nonhalogenated solvents (F003 and F004); and methyl ethyl ketone (F005).

3.6.1.2 Polychlorinated Biphenyl Waste Generation. PCB-contaminated TRU and LLW is generated by maintenance and periodic flushing of PCB hydraulic systems, failure of transformers and capacitors, and removal of PCB ballasts from light fixtures located in radioactive contaminated areas. The waste is packaged and shipped as solid waste to the CWC for storage.

Future generation of PCB-contaminated waste is expected to be variable. The generation of this waste stream is correlated with the failure rate of PCB transformers, capacitors, and fluorescent light ballasts. Additional generation may be related to general Hanford Site cleanup and decontamination/ decommissioning activities. Sitewide cleanup efforts may identify PCB-contaminated areas that will require cleanup and packaging.

\section{| 3.6.2 Characterization}

This section discusses waste characterization based on process knowledge and sample analysis, identifies known designations, and addresses any further characterization required or planned. 
Before any waste is accepted at the CWC, it is characterized and packaged as described in Hanford Site Solid Waste Acceptance Criteria (WHC 1993e). These criteria require that the generator of the waste characterize each individual container of waste with sufficient accuracy to permit proper segregation, treatment, certification, shipment, and storage.

3.6.2.1 Process Knowledge. The waste characteristics are determined by the waste generator based on documented knowledge of the process generating the waste or sampling, as appropriate. The generators of all waste shipped to the CWC are periodically audited to ensure that waste is being managed in accordance with Hanford Site Solid Waste Acceptance Criteria.

Process knowledge has been used to characterize PCB-contaminated TRU waste and LLW currently in storage. Equipment containing PCBs, such as hydraulic systems, transformers, capacitors, and fluorescent light ballasts have been identified clearly. These systems are managed in accordance with 40 CFR 761; waste is immediately handled and packaged as PCB TRU waste or LLW material.

1 3.6.2.2 Sample Analyses. The generators of all waste shipped to the $\mathrm{CWC}$ are audited periodically to ensure that waste is being properly characterized.

Hydraulic systems and transformers have been sampled to determine PCB concentrations. Any waste resulting from the management of these systems is designated based on the concentration of PCBs in the source system. Light ballasts are designated based on data from the manufacturers.

Additional sampling is planned when this waste is processed through either commercial or onsite treatment facilities.

3.6.2.3 Waste Designation and Basis. Waste at the CWC is designated based on the information provided by the generator. Waste designation is performed by the waste analysis organization as part of a waste acceptance evaluation in accordance with Hanford Site Solid Waste Acceptance Criteria (WHC 1993e), and recorded in the Solid Waste Information Tracking System database. This database includes Washington State and RCRA waste codes resulting from designations based on process knowledge and sample analysis. Waste codes have been entered into the database since 1988. When the waste codes were not found in database reports, waste designation tables were used to assign codes to containers placed in storage before 1988 . In the future, random physical verification will occur on verifiable waste streams. Waste streams I not verifiable by physical means will be verified by surveillance. (See Section 3.6.3.4 for details.)

3.6.2.4 Uncertainty of Waste Designation. The designation of the waste stored in the CWC is considered accurate.

3.6.2.5 Schedule for Further Characterization. No further characterization is required to accurately designate the present waste for storage. For some of the waste, additional characterization needs to be performed to determine proper treatment and disposal options. This characterization will be performed during processing at either commercial or onsite treatment 
facilities. Further characterization may be necessary for newly generated waste and/or as a result of changed regulations.

\section{| 3.6.3 Storage}

This section describes the storage units associated with the CWC and details the amount and characterization of the waste stored in these units.

| 3.6.3.1 Description of Storage Units and Capacity. The storage units described below are included in the CWC.

- Flammable Mixed-Waste Storage Modules-Twenty-three modules are operational to store flammable LLW, TRU waste, mixed LLW, and TRU-mixed waste with flash points below $38^{\circ} \mathrm{C}$. Two of these modules are used to store contaminated alkali metals. The total capacity is 2460.21 -cubic meter drums. The modules are small preengineered buildings with 16.3 square meters of floor space each.

- Mixed-Waste Storage Buildings - Thirteen mixed-waste-storage buildings are operational to store all categories of mixed waste (including TRU). The floor space of each building is 372 square meters. Each will have a 1,000-drum equivalent capacity. These facilities can store PCB wastes.

- Large Mixed-Waste Storage Facility-The large mixed-waste storage facility will be operational in five phases, from third quarter FY 1991 for Phase I through FY 1997 for Phase V. The large mixed-waste storage unit will store all categories of mixed LLW with a capacity of approximately 65,000 drum equivalents.

- Waste Unloading and Staging Area-This pad is 836 square meters in area and can hold approximately 2,500 drums stacked two high. This pad is not intended for long-term storage.

- Mixed-Waste Storage Pad-The mixed-waste storage pad is located adjacent to the radioactive mixed waste storage buildings and is used as an interim storage area.

- Mixed-Waste Disposal Trenches - The mixed-waste disposal trenches will be used to store treated mixed waste that require non-standard rigging and handling because of its of size or weight.

The planned capacity of the CWC to store LLW and TRU mixed waste is 17,908 cubic meters. This capacity includes 1996 construction and is adequate to store the current projected volumes of mixed waste to be generated through the year 2000 , assuming no treatment of the stored waste. Current plans call for treatment of the mixed waste to begin in 1999, which will reduce the amount of waste in storage and make storage room available for newly generated 
mixed waste. The capacity of the CWC to store mixed waste is continually evaluated and additional storage buildings will be constructed if necessary to meet forecast capacity shortfalls.

3.6.3.2 Amount in Storage. The amount of dangerous waste restricted from land disposal stored at the CWC as of June 1996 is 6,906 cubic meters. This includes 3,375 cubic meters of waste from the 183-H Solar Evaporation Basins.

I As of June 1996, 78.4 cubic meters of both RCRA and non-RCRA PCB TRU waste have been placed in the CWC for storage. Existing storage capacity is judged to be adequate for any future generation.

1 As of June 1996, 182 cubic meters of PCB LLW have been placed in the 2401-W Building for storage. Existing storage capacity is judged to be adequate for any future generation.

I 3.6.3.3 Storage Compliance Assessment. The CWC was reviewed for compliance with interim-status dangerous waste regulations during 1988.

The compliance assessment noted a specific area of noncompliance, the contingency plan. Compliance action schedules were developed as part of the Tri-Party Agreement (Ecology I et al. 1989). Interim-status compliance was achieved in June 1990.

\subsubsection{Treatment}

This section describes the treatment of mixed waste currently stored in the CWC.

3.6.4.1 Description of Current Treatment. Certain waste streams currently in storage in the CWC, such as the SY-101 air lances, AZ-101 thermocouples, and Battelle Columbus sludge, have characteristics amenable to existing small-scale onsite macroencapsulation and stabilization treatment capabilities. T Plant started treating these streams in FY 1996.

3.6.4.2 Description of Proposed Treatment. The other waste streams currently in storage in the CWC are not amenable to existing onsite treatments because of their size or physical characteristics. These waste streams will be treated by whichever of the following programs is best able to mitigate the hazards of each individual waste stream:

- WRAP 1

- Thermal Treatment Program

- Non-Thermal Treatment Program

- Milestone M-91 Program.

Table 3-6 provides a matrix that shows each major waste stream or group and its designated treatment unit. 
3.6.4.2.1 WRAP 1. WRAP 1, shown in Figure 3-7, became operational in March 1997. WRAP 1 will accept contact-handled TRU and LLMW in both box and drum forms. Capabilities are limited to nondestructive examination/nondestructive analysis (NDE/NDA) of boxes, but include NDE/NDA, segregation, decontamination, characterization, verification, and treatment of drummed waste. Services other than NDE/NDA are performed in the TRU and LLMW restricted waste gloveboxes. Waste treatment capabilities include neutralization, stabilization, amalgamation, macroencapsulation, and controlled reaction with water. Because of the way the gloveboxes are designed, waste throughput at WRAP 1 will be limited. A basic WRAP treatment diagram is shown in Figure 3-8.

3.6.4.2.2 Thermal Treatment Program. The thermal treatment program consists of two private and two DOE-complex facilities. Waste requiring thermal treatment will be evaluated on a stream-by-stream basis and assigned to the best alternative. The private facilities are the Allied Treatment Group (ATG) Richland thermal treatment facility and the Diversified Scientific Services, Inc. (DSSI) incinerator in Oak Ridge, Tennessee. The DOE complex facilities are the Waste Experimental Reduction Facility (WERF) incinerator at INEL and the Toxic Substances Control Act (TSCA) incinerator at Oak Ridge National Laboratory (ORNL).

ATG Richland Thermal Treatment Facility. The ATG Richland thermal treatment facility will be able to treat mixed waste and alpha-contaminated PCB waste in solid, absorbed liquid, and laboratory packed-liquid forms. Incoming waste will be segregated into two fractions, one that requires thermal treatment and the other that may be treated by less expensive lowtemperature methods. For the thermal treatment fraction, ATG will thermally destruct organic hazardous constituents and radionuclides in a grouted final form. The ATG facility is scheduled to begin treatment in FY 2001, and will have the capability to treat up to 717 cubic meters of waste per year.

DSSI Incinerator. The DSSI incinerator can treat combustible mixed waste liquids, in both containerized and bulk forms. The DSSI incinerator does not accept alpha-contaminated PCB waste. The DSSI does not provide the capability to segregate waste. The mixed waste liquids are blended with clean fuel and used to power an industrial boiler that provides steam for other non-related processes. The DSSI facility is currently operating. The DSSI radioactive materials license places significant limitations on the total inventory of radionuclides that can be processed per year.

WERF Incinerator. The WERF incinerator can treat combustible mixed waste in solid and absorbed liquid forms. The WERF incinerator does not accept alpha-contaminated PCB waste. The WERF incinerator provides limited capability to segregate waste into combustible and noncombustible fractions. Combustible fractions are packed into incinerable boxes and mechanically fed into a controlled-air design incinerator. Noncombustible waste is returned to the generator. The WERF incinerator is currently operating and has the capability to process up to 518 cubic meters of waste annually.

TSCA Incinerator. The TSCA incinerator can treat combustible mixed waste in solid and liquid forms. The TSCA incinerator does not accept alpha-contaminated PCB waste. The TSCA 
incinerator has a limited capability to segregate waste into combustible and noncombustible fractions. The TSCA incinerator is currently operating.

3.6.4.2.3 Non-Thermal Treatment Program. The Non-Thermal Treatment program was initiated after WRAP Module 2A was terminated in December 1994, and can treat waste originally assigned to WRAP $2 \mathrm{~A}$. The program consists of three components: commercial treatment, onsite treatment, and direct disposal. Waste that does not require thermal treatment will be evaluated on a stream-by-stream basis and assigned to the best alternative treatment.

Commercial Treatment. This component will procure treatment services to meet RCRA and WAC requirements. Targeted technologies include macroencapsulation for debris and elemental lead waste and stabilization for particulates, soils, and sludges. The treatment service may be performed at the vendor's site under the vendor's RCRA permit or may be performed by the vendor on site under the Hanford Site RCRA permit. Contracts will be placed on a streamby-stream basis, so that the best treatment process and location can be selected for each stream. Waste streams assigned to commercial treatment are generally those with large volumes that would require substantial capital investment or new facilities for effective treatment. Treatment will begin no later than September 1999.

Onsite Treatment. This component will use existing small-scale macroencapsulation, neutralization, and stabilization capabilities at $T$ Plant to treat specialty waste streams. This component is separate from amalgamation, neutralization, and stabilization treatment at WRAP 1, which was covered in Section 3.6.4.2.1. Waste streams assigned to onsite treatment are generally those with small volumes that would not require substantial capital investment or new facilities for effective treatment, or those that require significant pretreatment characterization and sorting (e.g., lab packs). This component is currently operating.

Direct Disposal. This component seeks to meet LDR treatment standards without additional treatment (RL 1995a). Typically, direct disposal efforts use sampling and analysis to demonstrate that waste meets LDR treatment standards or pursues regulatory relief of LDR treatment standards when technically advisable. Waste streams assigned to direct disposal are those where existing analytical data or process knowledge indicate that the concentration of hazardous constituents is very small or those where some type of treatment has already been performed.

3.6.4.2.4 Milestone M-91 Program. This program is the successor to the M-33 milestone discussions and will develop treatment capability for remote-handled and large-size TRU, TRUM, LLMW, and greater than Class 3 (GTC3) waste. Treatment alternatives will be evaluated by developing separate project management plans for TRU and TRUM and for LLMW and GTC3 waste. For each waste type, the preferred alternative developed in the project management plan will be constructed or procured. TRU and TRUM processing capability will start up no later than June 2005. LLMW and GTC3 treatment capability will start up no later than June 2008 .

3.6.4.3 Accelerated and Alternative Treatment. $\mathrm{RL}$ is pursuing alternative treatment requirements through the direct disposal team to minimize and thereby accelerate treatment. 


\section{| 3.6.5 Waste Reduction}

All plants and processes that generate waste that is shipped to the CWC are required to have a waste minimization program and a LLW certification plan in place. The effectiveness and implementation of these programs are audited on a regular basis. Key elements of this program are described in Section 2.5 .

\section{| 3.6.6 Variances, Treatment Equivalency Petitions, Time Extensions}

The CWC contains waste that is restricted from disposal because it contains solvents (40 CFR 268.30), Second-Third LDRs (40 CFR 268.34), and waste identified by the Third-Third LDRs (40 CFR 268.35).

The Tri-Party Agreement requires treatment and disposal capacity for this waste to be developed on the following schedule:

- Complete treatment and direct disposal of at least 1,644 cubic meters of contacthandled LLMW by September 2002 (Milestone M-19-00)

- Begin treatment of contact-handled LLMW by September 1999 (Milestone M-19-01).

If variances, exemptions, or time extensions are required because of delays in the development of treatment, storage, or disposal capacity or the demonstrated need for using alternative treatment technologies, they will be applied for in accordance with the procedures detailed in the Tri-Party Agreement or regulations.

The required treatment for certain PCB waste is incineration. Currently no facilities are available for incineration of mixed PCB waste. However, the thermal treatment contract facilities will be able to treat some of this waste. Alternative treatments currently are being investigated. The PCB waste will be stored at the CWC until an equivalent treatment technology is demonstrated and approved by EPA and Ecology. A compliance agreement between DOE-HQ and EPA-HQ approved in August 1996 allows storage of PCB-contaminated radioactive waste beyond 1 year.

A treatment equivalency petition has been approved by EPA and Ecology for formic acid in 183-H waste. The waiver allows for less costly stabilization treatment of the waste.

In February 1997, Ecology granted a "contained-in" determination for 828 drums of backlog waste soil from the tank farms. This determination allowed waste codes F001 through F005 to be removed from these drums. "State only" dangerous waste codes also are not required for PCBs in the waste, however all PCB soils are still required to be managed in accordance with TSCA. This determination was the result of review of analytical data from drum soil samples. 


\subsection{SUSPECT-TRANSURANIC AND TRANSURANIC WASTE AND OTHER REGULATED LLBG WASTE}

Since 1970 , defense materials production, research, and waste management have produced TRU waste. Before 1970 there were no regulations that defined or required separation of TRU waste and it was commingled and buried with LLW. Initially, the definition of TRU waste

I included any waste with suspect alpha contamination. In 1972 this definition was changed to include only waste containing greater than 10 nanocuries per gram of alpha-emitting isotopes with half-lives greater than 20 years. In 1982 the definition was again changed to include only waste with greater than 100 nanocuries per gram of TRU radionuclides. TRU radionuclides are those having an atomic number greater than 92 . Because existing technology in the 1970s could not determine the concentration of TRU radionuclides at 10 or even 100 nanocuries per gram, any solid waste that was suspected to be TRU was placed in retrievable storage (WHC 1989a) in the Low-Level Burial Grounds. The term retrievable storage is used to meet radioactive management requirements. RCRA defines this practice as disposal.

I The other regulated Low-Level Burial Ground waste stream that meets DOE radioactive management requirements consists of waste that was generated after 1980 and in 1987 or before, 1 when placement in Low-Level Burial Ground retrievable storage was terminated. The waste contained liquid organics that precluded disposal as solid LLW because of concerns about affecting the ion exchange capacity of the soil. This waste is stored in retrievable storage units in the same manner as retrievably stored TRU waste.

The retrievably stored waste at the Hanford Site was not segregated based on the physical or chemical characteristics of the waste. The waste containers are filled with mixtures of materials, such as failed process equipment including pumps, resin columns, and tanks; laboratory and room trash including paper, plastics, glassware, cloth, solidified liquids, and animal carcasses; and decontamination and decommissioning rubble including concrete, piping, and soils.

The waste is contained primarily in 0.21-cubic-meter drums and metal or wood boxes. Waste is also contained in casks, concrete boxes, concreted culverts, and other miscellaneous containers.

Before 1986, TRU waste had been placed in a variety of configurations with the intent of excavating this waste in the future. These storage configurations consisted of shallow land trenches, concrete-lined " $V$ " trenches, and earth-covered asphalt pads and caissons. The TRU I waste has been stored in the TRUSAF since 1986 (Section 3.8) and in the CWC since 1987 I (Section 3.6).

The majority of the TRU waste stored in the 200 Areas is generated by onsite activities; however, some of the TRU waste is generated off site and shipped to the Hanford Site for retrievable storage (RHO 1985). Approximately 15,000 cubic meters of TRU waste had been | placed in retrievable storage in the 200 Areas in more than 38,700 containers. 
In addition to the waste stream that will be generated on excavation, the Hanford Facility has received submarine reactor compartments for disposal in the Low-Level Burial Grounds TSD unit. The submarine reactor compartments are placed in the 200 East Area Burial Ground 1 218-E-12B, Trench 94. These defueled reactor compartments are disposed of, without further treatment, in their current location. For this reason, the compartments are not included in the storage inventory tables, waste minimization sections, or treatment discussions of this report. As much of the PCBs and lead as practical have been removed from the submarine reactor compartments. The remaining lead and PCBs are encapsulated within the sealed hulls of the compartments.

As of March 1997, 62 reactor compartments were disposed of. Additional reactor compartments will be shipped to the Hanford Site in the future.

A pilot project to excavate and retrieve 198 drums from these locations was started in 1994. To date, 171 drums have been retrieved, inspected in place, or stored at the CWC. Data on the integrity of the drums have been compiled.

\section{| 3.7.1 Generation}

Extensive process knowledge is not available for many of the containers that will be excavated or retrieved. The best available information is maintained in the computerized Solid Waste Information and Tracking System database. The Solid Waste Information and Tracking System contains information provided by the waste generator. In the past, exhaustive waste descriptions that could be used to classify a waste accurately were not required and data entries such as "contaminated debris" and "mixed fission products" were common (WHC 1989a). Because of incomplete classification of waste in the past, it is estimated that 10 percent of the TRU waste may be mixed waste.

\section{3.7.2 Characterization}

This section discusses waste characterization based on process knowledge and sample analysis, identifies known designations, and addresses any further characterization required or planned.

I 3.7.2.1 Process Knowledge. Limited process knowledge has been used to characterize the suspect-TRU and TRU mixed waste that will be excavated or retrieved. In the past few years changing waste reporting, manifesting, and packaging requirements have greatly increased the availability of process waste data for what may be used to characterize waste. Information related to the physical, chemical, and radiological properties of newly generated TRU waste is available. This availability is anticipated to reduce the amount of sampling and treatment required to meet long-term storage packaging requirements. 
3.7.2.2 Sample Analyses. Sampling for mixed waste constituents will be performed when the TRU waste is retrieved from storage for processing. All drums and boxes of suspect-TRU waste in interim storage will be opened. Selected containers will be sampled and these samples will be prepared for transport to analytical laboratories in the 200 West Area for analysis.

1 3.7.2.3 Waste Designation and Basis. A review of data on suspect-TRU and TRU waste units identified many constituents as designated dangerous waste. Data entered since 1988 have the designation of the dangerous constituents of each waste package assigned. When the designation was not found in database reports, waste designation tables were used to assign a designation to the constituents identified in waste placed in storage before 1988 .

It is anticipated that additional TRU mixed waste will be identified when waste is retrieved from storage for repackaging for disposal (WHC 1989a).

I 3.7.2.4 Uncertainty of Waste Designation. There is high confidence in the accuracy of the designations for newly generated TRU waste material. Older waste will require additional characterization before treatment and disposal.

1 3.7.2.5 Schedule for Further Characterization. In situ sampling of the suspect-TRU waste was initiated in FY 1991 (WHC 1989b). The purpose of the sampling is to assess the current and future integrity of the retrievably stored waste containers and analyze contents. These objectives will be achieved by visual and nondestructive examination of waste containers, retrieval, and nondestructive assay.

The suspect-TRU and TRU pilot retrieval and inspection program conducted in 1994 laid out a three-step approach to gather and provide data on the TRU waste at the Hanford Site for the WRAP facilities and the DOE complex. The first step evaluated existing TRU waste records to assess completeness of waste characterization data. The existing written data lacked detail and little physical data were available to support a full-scale excavation and retrieval program. Therefore, the second step was undertaken to physically retrieve and inspect stored TRU waste, while a third step would fully characterize the retrieved waste.

Locations for excavation, retrieval, and inspection were chosen based on waste storage configurations, waste generator records, radioisotope distribution, radiation dose, age of waste, and several other parameters. Inspection equipment was developed to ultrasonically inspect TRU waste drum integrity. Retrieval equipment was developed to lift the unearthed drum from the storage trench, vent the drum, and sample the drum gas for analysis. The entire program would evaluate TRU waste storage at several different trench locations and provide the needed retrieval, inspection, and characterization data to the WRAP project and other DOE sites.

Excavation, retrieval, and inspection of TRU waste drums at two sites is complete. (No other retrieval operations are planned beyond these sites.) Twenty-three TRU drums that were placed between 1977 and 1980 were retrieved for characterization and examination. The retrieved drums have been radiographed to compare contents against waste records. Radioassay of the drums was also conducted and yielded a \pm 50 -percent assay accuracy (total plutonium) 
when compared to the original assay records. Drum head-gas sampling was conducted on 10 vented drums after an 8-day collection period. Elevated total volatile organic (VOC) readings were found in each sample ranging from 84 to $517 \mathrm{ppm}$. When tests were conducted to determine the composition of the organic compounds more than 23 compounds were revealed. It appears that the individual compounds are not above the reportable limits in WAC 173-303. This determination is based only on the head-gas sample. When the containers are opened and the soil is sampled, the results may be different.

Ninety drums were ultrasonically inspected by taking almost 1,700 separate ultrasonic readings. Drums also were examined in situ using a remote viewing camera. In all, 171 drums were examined between the two sites. Three drums contained areas of concern and were overpacked and left in the trench. One drum had two small holes in the side. The area was patched and the drum was stabilized and left in the module in accordance with safety documents and procedures. In addition, ground-penetrating radar was used to locate underground drums in all 19 sites identified for retrieval, and its accuracy was evaluated in the two sites entered. The retrieval and inspection of these drums was terminated as inclement weather set in.

Additional sampling will be performed as necessary to adequately characterize suspected mixed waste when waste packages are excavated or retrieved and processed through the WRAP 1 facility.

\section{| 3.7.3 Storage}

This section describes the current inventories of TRU and suspect-TRU waste that will be excavated or retrieved. RCRA considers the placement of such waste in the Low-Level Burial Grounds as disposal and not storage. DOE radioactive waste management requirements classify this practice as retrievable storage.

3.7.3.1 Storage Unit and Capacity. The waste stream primarily consists of 0.21 -cubic-meter drums and boxes. Initially drums were painted; however, after 1982, galvanized drums were used to minimize corrosion attributed to high humidity in the locations in which they were placed. Initially boxes were constructed of plywood and steel, later of plywood coated with fiberglassreinforced polyester, and currently of steel. Waste also is contained in casks, concrete boxes, concreted culverts, and other miscellaneous containers. These containers were placed in a variety of storage configurations. These storage configurations consisted of shallow land trenches, concrete lined "V" trenches, and earth-covered asphalt pads and caissons (Figures 3-9 and 3-10).

Suspect-TRU and TRU waste is located in the 218-W-3A, $-4 B,-4 C$, and 218-E-12B Burial Grounds. Newly generated (after 1987-1988) TRU waste has been stored in the TRUSAF and CWC storage buildings.

Four different container storage configurations were used for the retrievably stored contact-handled TRU waste at the Hanford Site. The first configuration consists of waste drums 
stacked horizontally in a gravel-bottom "V" trench. The waste drums were covered directly with soil. This storage configuration was used from 1970 through 1972.

The second storage configuration was an engineered concrete and metal storage structure known as the V-7 trench. In the V-7 trench, drums were stacked on a 45 -degree angle. This storage concept proved too expensive to implement and was used only between June 1972 and March 1973.

The third configuration consists of wide bottom and " $V$ " trenches. In both cases it is unknown if the trench floor was covered with plywood and drums were stacked vertically or if it I was placed similar to Configuration 1 (Figure 3-10). Boxed waste in this configuration may contain shoring used to protect it from collapse because of soil pressure. This configuration was used in the 200 West Area 218-W-3A and 218-W-4B Burial Grounds starting in 1974.

The fourth configuration consists of wide-bottom trenches. This storage configuration is the same as the third except the floor is asphalt. This storage configuration was used in the 218-W-4B Burial Ground, trench 07, from 1974 until 1980 and in the 218-W-4C Burial Ground from 1978 to the present.

Some of this waste is remote-handled waste shielded for contact handling. In addition, small containers of remote-handled TRU waste are stored in buried caissons; these caissons no longer are used for newly generated waste. The caissons are reinforced concrete cylinders 2.7 meters in diameter by 3 meters high and are buried 4 meters below grade. The caissons have 0.9-meter-diameter inlet chutes, offset or convoluted to reduce radiation or "shine" from the contents (Figure 3-10). Caissons are equipped with electrically driven exhausters fitted with HEPA filters.

3.7.3.2 Amount of Waste Placed. Approximately 15,440 cubic meters of waste have been placed in the 200 Areas. Of this volume, 14.1 percent or 2,184 cubic meters are known to be dangerous waste based on information contained in the Solid Waste Information and Tracking System. Additional waste may be redesignated as dangerous, land disposal restricted waste on excavation or retrieval.

I 3.7.3.3 Storage Compliance Assessment. Because RCRA considers this practice as disposal, this section is not applicable.

Compliance action schedules were developed as part of the Tri-Party Agreement (Ecology I et al. 1989). Compliance with contingency plan upgrade, inspection, and labeling requirements was achieved by June 1990 . Use of cardboard boxes for burial was terminated effective January 1990. Processing facilities for compatible wastes are currently available. Additionally, two groundwater monitoring wells were installed in 1993 for a total of 81 wells in the low-level burial grounds. Detailed information on these wells, cuttings, purgewater, and characterization data can be found in the Borehole Completion Packages for the year in which the wells were completed (WHC 1991f, WHC 1992d, WHC 1994c). 
The Part B Permit application, which documents the then-current compliance status with I the dangerous waste regulations, was submitted in October 1991.

\section{| 3.7.4 Treatment}

This section describes the current and proposed treatment of excavated suspect-TRU and TRU waste.

\subsubsection{Current Treatment. No waste is being treated.}

3.7.4.2 Proposed Treatment. Excavated suspect-TRU and TRU waste from retrievable storage units will be retrieved and shipped to the WRAP Facility, Module 1 or a commercial facility for treatment of waste so that it is acceptable for permanent disposal. Treatment activities include segregation of LLW and TRU waste from hazardous waste, repackaging waste, conducting nondestructive examination and nondestructive assaying of packaging, and certifying packages for shipment and disposal.

The WRAP facility was proposed to be constructed as three modules with Module 1 operations to begin in March 1997. Module 2A has been canceled with commercial contracts providing the treatment capacity. Operations are planned to begin in 1999. Module 2B is | currently being reviewed as part of Milestone M-91-00.

\section{| 3.7.5 Waste Reduction}

The locations where the suspect-TRU and TRU waste is placed no longer accept waste; therefore, a waste minimization program is not applicable. However, waste minimization will be considered when evaluating cleanup and disposal alternatives.

\section{| 3.7.6 Variances, Treatment Equivalency Petitions, Time Extensions}

The waste placed in these units after 1982 may be restricted from land disposal because it contains spent solvent waste if process knowledge identifies the spent solvent listing as applicable when the waste is retrieved. In addition, California List waste, characteristic waste, or state-only criteria waste designations may cause additional disposal restrictions.

The Tri-Party Agreement requires treatment and disposal capacity wastes to be developed on the following schedule:

- Completion of WRAP Facility, Module 1, required to sort and repackage waste, and initiation of operations by March 1997 (Milestone M-18-00, completed) 
- Completion of WRAP Facility, Module 2A, required to provide waste treatment capabilities that minimize the land disposal of low-level radioactive and mixed waste by September 1999 (Milestone M-19-00). This facility has been canceled; commercial options are being pursued to meet the schedule stipulated in M-19-00.

If variances, treatment equivalency petitions, or time extensions are required because of delays in the development of treatment, storage, or disposal capacity or the demonstrated need for using alternative treatment technologies, they will be applied for in accordance with the procedures detailed in the Tri-Party Agreement or regulations.

\section{| 3.8 TRANSURANIC WASTE STORAGE AND ASSAY FACILITY STORED WASTE}

TRU solid waste packaged in compliance with the current Hanford Site Waste Acceptance Criteria (WHC 1993e) is stored in the 200 West Area, in the 224-T Building, also known as the TRUSAF, as well as the CWC.

\section{| 3.8.1 Generation}

The following are descriptions of current sources of TRU mixed waste.

- The PUREX Plant reprocessed irradiated fuel from N Reactor. Radioactive solid waste collected from the PUREX Plant consists of room waste, such as gloves, paper, and plastics. The TRU portion is separated from the LLW. Some of the waste, such as mercury-filled light tubes, rags, and aerosol cans, are definitely dangerous and separate collection receptacles are established for collecting this waste. To ensure that dangerous waste is not inappropriately discarded with the LLW or TRU waste, the waste is sorted before packaging and shipment.

- The PFP routinely generates mixed solid waste. Fluorescent light tubes containing mercury are used in processing gloveboxes and radiation areas throughout the PFP. The majority of PCB ballasts and fluorescent light tubes are surveyed for radiological contamination and released. These waste streams are handled as hazardous waste. A small portion of the ballasts and fluorescent light tubes are radiologically contaminated and must be treated as mixed waste. Lead-lined gloves on processing gloveboxes are routinely replaced to minimize the potential for glove failure and subsequent spread of radioactive contamination. Laboratory waste containing xylene and toluene are generated during the analysis of samples for neptunium and plutonium. The waste is packaged and shipped as solid waste.

- Operations of the analytical laboratories in the 200 West Area generate small quantities of TRU mixed waste. Included in this mixed waste is radioactively contaminated lead, outdated solid commercial chemicals, and lead-shielded waste from laboratory hot-cell operations. 
- The PNNL generates small quantities of TRU mixed waste from research operations that are fully characterized by process knowledge.

The TRUSAF received some containers of waste from offsite sources (such as Battelle Columbus, Ohio; Argonne National Laboratory, Chicago; Rocky Flats Plant, Colorado; and Lawrence Berkeley Laboratories, California). These containers were sent to TRUSAF for storage before their planned shipment to the WIPP. The TRUSAF only accepted waste that was packaged in 0.21-cubic-meter drums. Newly generated TRU waste is stored in the CWC. There is a moratorium on receipt of offsite TRU waste shipments. The Governor of Washington submitted a letter to the Secretary of Energy stating that no TRU waste shipment into Washington State will be accepted until the WIPP is opened. Action on this matter is pending by DOE-HQ.

\section{| 3.8.2 Characterization}

This section discusses waste characterization based on process knowledge and sample analysis, identifies known designations, and addresses any further characterization required or planned.

To be accepted at TRUSAF, waste was packaged and characterized as described in the Hanford Site Solid Waste Acceptance Criteria (WHC 1993e). These criteria require that the generator of the waste characterize each individual container of waste with sufficient accuracy to permit proper certification, shipment, and storage. Kinds and quantities of dangerous constituents in the waste and physical and chemical characteristics of the waste must be known and recorded on appropriate forms.

| 3.8.2.1 Process Knowledge. The waste characteristics are determined by the waste generator based on documented knowledge of the process generating the waste. The generators of all I waste shipped to TRUSAF and the CWC are periodically assessed to ensure that waste is being properly characterized. Currently, only four facilities (PUREX, PFP, PNNL, and the spent fuel | program) are able to certify TRU waste.

| 3.8.2.2 Sample Analyses. Samples are collected at the point of generation for any sample analysis required to adequately characterize for waste designation. No samples are collected at TRUSAF. Any waste that requires sampling will not be certified and consequently will be shipped to the CWC for storage and subsequent processing.

1 3.8.2.3 Waste Designation and Basis. The dangerous waste designation of each waste container is determined at its point of generation based on knowledge of the waste placed in the container.

| 3.8.2.4 Uncertainty of Waste Designation. The designations of waste stored in TRUSAF are considered to be accurate. 
| 3.8.2.5 Schedule for Further Characterization. Certified waste in interim storage is awaiting shipment to the WIPP. No further characterization is required for this waste.

\section{| 3.8.3 Storage}

This section addresses current storage units, describes inventories, and assesses compliance with applicable regulations.

3.8.3.1 Storage Unit and Capacity. The 224-T TRUSAF building was originally constructed in 1943 to purify plutonium nitrate by the lanthanum fluoride process; it was idle for several years after new technology made it obsolete. In the early 1970 s, the building was modified to meet requirements for storage of plutonium-bearing scrap and liquids. The cells in the processing areas have been completely sealed and isolated from the operating gallery and service areas. These operating and service areas have been stripped of all unnecessary control equipment, panel boards, and partitions to provide approximately 1,068 square meters of storage space on three f floors (Figure 3-11). The unit storage capacity is 420 cubic meters (2,000 drums).

The CWC now accepts TRU waste and no additional waste will be shipped to TRUSAF.

3.8.3.2 Amount in Storage. As of June 30, 1996, 257 cubic meters of TRU mixed waste are stored in TRUSAF.

3.8.3.3 Storage Compliance Assessment. The TRUSAF unit was reviewed for compliance with interim-status dangerous waste regulations during 1988. The need for an upgraded contingency plan was identified and the plan was completed. A Part B Permit application has been submitted.

\subsubsection{Treatment}

This section describes the current and proposed treatment of stored TRU waste.

| 3.8.4.1 Current Treatment. At TRUSAF, packaged waste was $x$-rayed (to ensure that what can be identified generally agrees with the documentation) and assayed to determine TRU activity. All TRU waste packages that meet the WIPP/Waste Acceptance Criteria requirements | were placed in interim storage pending shipment to the WIPP. LDR treatment for TRU mixed I waste is not be required for disposal in WIPP. Noncertifiable TRU waste was sent to the CWC or stored in the TRUSAF. When the WRAP Facility, Module 1, begins operating, nondestructive evaluation and assay activities will be transferred from TRUSAF to the WRAP facility.

1 3.8.4.2 Proposed Treatment. Certified TRU waste in TRUSAF interim storage will be shipped to the WIPP for permanent disposal.

3.8.4.3 Treatment Alternatives. The certified TRU waste is not planned to be treated. Noncertified TRU waste will be processed to meet WIPP waste acceptance criteria. Processing will 
include segregating LLW and TRU waste from hazardous waste, repackaging waste, conducting nondestructive examination and nondestructive assaying of packaging, and certifying packages for shipment and disposal. The WIPP facility will be the only facility in the nation capable of permanent disposal of this waste.

3.8.4.4 Accelerated Treatment. Current plans are to ship the waste to WIPP for permanent disposal. Acceleration of shipment to WIPP is not possible because WIPP has not yet opened.

\section{| 3.8.5 Waste Reduction}

All plants and processes that generate waste that is shipped to TRUSAF (now CWC) are required to have a waste certification program in place. The effectiveness and implementation of this program is audited regularly. Key elements of this program are described in Section 2.5 .

\section{| 3.8.6 Variances, Treatment Equivalency Petitions, Time Ertensions}

If variances, treatment equivalency petitions, or time extensions are required because of delays in the development of treatment, storage, or disposal capacity, they will be applied for in accordance with the procedures detailed in the Tri-Party Agreement or regulations.

\section{| 3.9 324 RADIOCHEMICAL ENGINEERING CELLS WASTE}

The 324 REC is located in the 324 Building in the 300 Area of the Hanford Site. It consists of four hot cells (A, B, C, and D) located around a central airlock.

The 324 Building has been used in numerous DOE-sponsored research and development programs since the mid-1960s. The major activities that have influenced the generation of mixed waste include:

- The Waste Solidification Engineering Prototypes Program (completed 1972)

- The development of treatment technologies via the Nuclear Waste Vitrification Project for wastes from Spent Nuclear Fuel reprocessing (1979)

- A pilot-scale Radioactive Liquid-Fed Ceramic Melter testing program in conjunction with the Federal Republic of Germany (1984-1987).

A closure plan for this facility has been prepared (PNNL 1995). 


\section{| 3.9.1 Generation}

This section describes how the waste in the 324 REC was generated. Most of the materials I now in the REC accumulated during research activities from 1965 to 1987 , except for solid 1 residues from the treatment of HLV tank waste. Over the 20+ years of these engineering demonstrations, equipment (such as tools, manipulator boots, and construction materials) were dropped and liquids (such as feed materials and samples) leaked onto the floor. In addition, particulate materials (essentially dust) introduced with normal air flow into the cell became contaminated.

Operational protocols in the REC were based on the radioactive properties of these materials. These materials were secured within the cell and did not interfere unduly with engineering operations. Because of technical difficulties, funding constraints, and safety issues associated with consolidating and/or retrieving, packaging, and/or transporting the waste materials, they were left in place.

Cleanout of the hot cells to eliminate the unacceptable radiological hazards associated with the dispersible material in the B Cell began in 1988 with completion estimated by 2000 .

No further generation of waste, other than used HEPA filters, is anticipated. Current waste types contained within the REC and their estimated volumes are provided below.

- Approximately 1.0 cubic meter of tools, equipment, and pieces of metal dropped on the floor during operations; dust and particulates contaminated with sporadically released material (feed solution that contained heavy metals) from process equipment.

- Approximately 1.02 cubic meters of waste elemental lead, used as shielding and counterbalances. Some of this may eventually be cleaned and reused or recycled during the cleanout of the hot cells.

- Approximately 0.6 cubic meter of filters loaded with solid residues resulting from treatment of the $\mathrm{HLV}$ tank waste.

\subsubsection{Characterization}

This section discusses the best available waste characterization information.

| 3.9.2.1 Process Knowledge. Based on process knowledge, none of the designated mixed waste in the REC is currently classified under RCRA as "listed" hazardous waste. The waste types characterized solely on the basis of process knowledge are as follows.

- Waste elemental lead ( 0.80 cubic meter)-D008

- HLV tank waste treatment residue (0.6 cubic meter) -D005 
I 3.9.2.2 Sample Analyses. In 1995, results of laboratory analyses of some waste became available. The following waste types are characterized on the basis of analytical data.

Dispersible debris ( 2.5 cubic meter of tools, equipment, metal pieces, dust, and particulates)--D006, D007, D008

I 3.9.2.3 Waste Designation and Basis. The basis for the designation of the 324 REC waste is process knowledge, supported by analytical data when available.

| 3.9.2.4 Uncertainty of Waste Designation. The designation of waste types in the REC is considered accurate.

| 3.9.2.5 Schedule for Further Characterization. No further waste characterization is planned.

\section{| 3.9.3 Storage}

The 324 REC does not receive any waste from other sources. It only stores waste that was generated from the operations conducted within the REC itself. The volumes currently in storage are provided in Section 3.9.1

3.9.3.1 Storage Unit and Capacity. The 324 REC is located in the 324 Building and consists for four hot cells (A, B, C, and D) located around a central airlock. The 324 Building was constructed to strict nuclear standards to safely house operations involving highly radioactive materials and to prevent releases to the environment.

The capacity for mixed waste storage of the hot cell section available for mixed-waste storage is estimated to be 15 cubic meters. This estimate is based on the past inventory in storage ( 9.17 cubic meters) and the consideration that no future significant generation of waste at this facility is anticipated.

3.9.3.2 Amount in Storage. A detailed explanation of the volumes currently in storage is provided in Section 3.9.1.

3.9.3.3 Storage Compliance Assessment. The high radioactivity field in most of the 324 REC precludes manned entry into the cells. As a result, certain Ecology requirements (e.g., use of written labels, physical inspection, closure by removing all wastes to background levels) are not practicable. In an effort to bring the $324 \mathrm{REC}$ into compliance, negotiations with Ecology and the EPA took place as part of the 1994 Tri-Party Agreement negotiations sessions. A compliance agreement was negotiated with and approved by Ecology. The 324 REC is in compliance with the terms of this agreement. Milestone M-89 includes both interim and final action to complete the closure of the $324 \mathrm{REC}$. 


\section{| 3.9.4 Treatment}

Currently stored waste in the 324 REC unit is not being treated. Sections 3.9.4.1 through 3.9.4.4 cover treatment options for waste stored in the 324 REC.

3.9.4.1 Current Treatment. Currently stored waste in the $324 \mathrm{REC}$ unit is not being treated.

3.9.4.2 Proposed Treatment. No treatment of 324 REC waste is proposed at the 324 REC.

I 3.9.4.3 Treatment Alternatives and Accelerated Treatment. Milestone M-89-02 provides for removal of all REC B-Cell mixed waste and equipment by May 31, 1999. Some REC mixed waste will be shipped to the PUREX tunnels and the CWC for interim storage. Waste will be treated in accordance with the proposed treatment scenarios for these units.

\section{| 3.9.5 Waste Reduction}

Waste reduction has been accomplished through waste segregation. Waste volume has been reduced by approximately 35 percent during cleanout of the REC. Also, analytical data have shown that 50 percent less waste is present than was previously believed present.

\section{| 3.9.6 Variances, Treatment Equivalency Petitions, Time Extensions}

If variances, treatment equivalency petitions, or time extensions are required because of delays in the development of treatment, storage, or disposal capacity, they will be applied for in accordance with the procedures detailed in the Tri-Party Agreement or regulations.

\section{| 3.10324 HIGH-LEVEL VAULT TANK WASTE}

The $324 \mathrm{HLV}$ is located in the 324 Building in the 300 Area of the Hanford Site. It consists of four tanks inside a shielded vault. The vault is lined with stainless steel and is

I equipped for leak detection. Tanks TK-104, TK-105, and TK-107 contained mixed waste that was generated from materials left over from research operations.

\section{| 3.10.1 Generation}

The mixed waste in the $324 \mathrm{HLV}$ was generated when process solutions remaining in the tanks from research operations were no longer needed. The waste formerly in the $324 \mathrm{HLV}$ was treated and the treatment residues were transferred to 324 REC, B-Cell in 1996. 


\subsection{B PLANT CELL 4 WASTE}

Drums with a 208-liter capacity containing both mixed waste and LLW generated in the WESF hot cells are stored in the B Plant Cell 4 storage area. This is allowed in the B Plant Part A Permit. Currently, the Cell 4 storage area contains 5 drums of mixed waste and 30 drums of highly radioactive $L L W$. The sole hazardous constituent in the mixed waste drums is lead solder on incandescent lamps from the hot cells. Because the lamps were packed into drums with other LLW waste because of space constraints in the hot cells, the entire drum is managed as mixed waste.

\subsubsection{Generation}

Since 1988, waste generated in the WESF hot cells has been packaged into 208-liter drums and transferred to B Plant for storage in Cell 4. When lights in the hot cell were changed, the bumed out lamps were packaged into the drums along with all the other waste generated. These transfers were performed frequently until 1991, but only a few have occurred since then. Current plans to clean out the hot cells may result in a resumption of such transfers to Cell 4 . Agreements with Ecology reached during transition negotiations under the Tri-Party Agreement (Ecology et al. 1989) will allow this process to continue through the deactivation of the facility.

\subsubsection{Characterization}

This section discusses the waste characterization and its basis. The waste designation, the uncertainty of the designation, and the schedule for further characterization also are provided.

3.11.2.1 Process Knowledge. The quantity of lead solder on the WESF hot cell incandescent lamps is known from contacts with the vendor who supplies the lamps. Combined with operating experience, determining the total quantity of lead solder in the waste matrix and making a book designation of the waste is possible. Inventories of the waste are prepared while the drum is packaged in the hot cell.

3.11.2.2 Sample Analyses. The waste in Cell 4 has not been sampled or analyzed. Because of the high radiation levels associated with this waste and the high degree of process knowledge, sample analysis was not deemed necessary to accurately characterize and designate the waste.

3.11.2.3 Waste Designation and Basis. The waste is designated TCLP toxic for lead (D008). This is consistent with past guidance issued by the Site Environmental Services group for incandescent lamps. Based on results from multiple samples for waste matrices that include lead solder, including similar incandescent lamps, these lamps are likely to yield an extract containing more than 5.0 milligrams per liter of lead when exposed to a leachate.

3.11.2.4 Uncertainty of Waste Designation. The designated waste codes for the B Plant Cell 4 waste are considered accurate. 
3.11.2.5 Schedule for Further Characterization. No further characterization of this waste has been scheduled.

\subsubsection{Storage}

This section describes the storage units and provides an update of its compliance status.

3.11.3.1 Description of Storage Unit and Capacity. Cell 4 is located in the B Plant 221B canyon building. It is approximately 8 meters by 4 meters by 6 meters deep. It stores the waste drums on a wooden platform that can hold up to 59 drums. Additional platforms can be constructed and mounted on top of the existing platform to create additional storage capacity. A maximum of 245 drums or 51 cubic meters can be stored in Cell 4 in this configuration. Cell 4 is covered by concrete cover blocks to isolate it from the canyon deck.

3.11.3.2 Amount in Storage. Five drums (1 cubic meter) of mixed waste are currently being stored in Cell 4 . In addition, 30 drums of LLW are also being stored in the area. This leaves about 24 empty spaces on the existing platform. Additional waste from the WESF hot cells may be stored in Cell 4 during deactivation.

3.11.3.3 Compliance Status. Cell 4 is a permitted container storage TSD unit. In accordance with agreements reached during Tri-Party Agreement negotiations on facility transition, Cell 4 will be allowed to continue receiving WESF hot cell waste through facility transition. A preclosure work plan with complete waste inventories and descriptions will be submitted to Ecology in accordance with Tri-Party Agreement Milestone M20-21A.

\subsubsection{Treatment}

Treatment has not been planned or scheduled for this waste. Current plans call for the waste disposition to be addressed during final facility closure. According to 40 CFR 268, the required treatment for radioactive lead solids is macroencapsulation.

\subsubsection{Waste Reduction}

Current plans are to clean out and shut down the majority of the WESF hot cells. This will prevent any additional waste from being stored in Cell 4.

\subsubsection{Variances, Treatment Equivalency Petitions, Time Extensions}

Removal of the mixed waste remaining in B Plant's Cell 4 will be addressed at a later date. The Tri-Party Agreement allows the mixed waste to be left in this storage area through the facility deactivation process. 
If variance, treatment equivalency petitions, or time extensions are required because of delays in the development of treatment, storage, or disposal capacity, they will be applied for in accordance with the procedures detailed in the Tri-Party Agreement or regulations.

\subsection{B PLANT CONTAINMENT BUILDING STORAGE}

The B Plant Containment Building consists of the 221B canyon area and the process cells inside the canyon. Waste, primarily discarded process equipment and jumpers, stored on the canyon deck and in the actual process cells is considered to be stored in the containment building. The containment building is a permitted TSD unit. On September 30,1994, the storage designation was changed from "waste pile" to "containment building."

\subsubsection{Generation}

The lead in the B Plant containment building consists of material that has been used as shielding or counterweights for various process equipment or jumpers in the $221 \mathrm{~B}$ canyon. In most cases, this lead is encased in steel. The primary source is from counterweights and jumpers, which are rigid lengths of pipe used as transfer lines to move process liquids between tanks and vessels and throughout the canyon. Determining exactly how many jumpers actually use lead counterweights is difficult, but up to 7,500 kilograms of lead may be present in this form.

Significant quantities of additional waste are not expected to be generated for storage in the containment building. Primary efforts will be focused on continued identification of the existing waste. Some additional waste may be generated during facility deactivation.

\subsubsection{Characterization}

This section discusses the waste characterization and its basis. The waste designation, the uncertainty of the designation and the schedule for further characterization also are provided.

3.12.2.1 Process Knowledge. The amount of lead in each process jumper is known from design drawings, as is the amount of shielding for pieces of process equipment.. In addition, historical process flow diagrams and operating knowledge provide a basis for determining what types of waste were processed using this equipment. The waste being stored on the canyon deck and in the process cells has been inventoried.

3.12.2.2 Sample Analyses. No sampling and analysis has been performed on the waste in the B Plant containment building. None will be performed during deactivation of the facility. Process knowledge will be used to adequately characterize the waste that will remain in the containment building during deactivation. 
3.12.2.3 Waste Designation and Basis. Some of the waste has been designated as TCLP toxic for lead (D008) because of the lead used as a component of the equipment. In addition, F001 through F005 waste codes have been applied to those pieces of equipment that were used to manage or process listed DST and/or SST waste. Heavy metals waste codes (D004-D011) may also apply in some instances because of potential waste residue on process equipment.

3.12.2.4 Uncertainty of Waste Designation. Because solid sample analysis information is lacking, some uncertainty is associated with the heavy metal waste codes (D004-D011). Waste designations for D008 and F001 through F005 are considered accurate based on process knowledge.

3.12.2.5 Schedule for Further Characterization. No further characterization of this waste has been scheduled. Additional characterization may be performed during deactivation to identify mixed waste that will remain in the containment building during final facility closure activities.

\subsubsection{Storage}

This section describes the storage units and provides an update of its compliance status.

3.12.3.1 Description of Storage Unit and Capacity. The B Plant canyon is approximately 260 meters long by 21 meters wide by 22 meters high. It is a concrete structure with walls several feet thick. Waste stored in the containment building is protected from the external environment. No free liquids are stored in the containment building. Waste stored in the process cells are further protected by large concrete cover blocks that enclose the cells and form the canyon deck. Full capacity of the containment building is as much as 35,000 cubic meters, but only the area on the deck itself can realistically be used for storage. Waste is not stacked or piled to use the free air space.

3.12.3.2 Amount in Storage. Accurate estimates of the total quantity of waste currently stored in the containment building are not available. An inventory of the existing waste in the process cells and on the canyon deck has been developed and is maintained.

3.12.3.3 Compliance Status. The B Plant containment building is a permitted storage TSD unit. In accordance with agreements reached during Tri-Party Agreement negotiations on facility transition, additional containment building waste may be generated and stored through facility transition. A preclosure work plan with complete waste inventories and descriptions will be submitted to Ecology in accordance with Tri-Party Agreement Milestone M20-21A.

\subsubsection{Treatment}

Treatment has not been planned or scheduled for this waste. Current plans call for the waste disposition to be addressed during final facility closure. The specified LDR treatment for radioactive lead solids is macroencapsulation. Hazardous debris treatment that takes place in the 
permitted containment building and meets the standards specified in 40 CFR 268.45 is also acceptable.

\subsubsection{Waste Reduction}

Deactivation efforts at the facility are drastically reducing the number of operational systems. As a result, significantly fewer process jumpers will be generated. In general, most of the waste that will be stored in the containment building is already there. Identifying and categorizing it remains to be done.

\subsubsection{Variances, Treatment Equivalency Petitions, Time Extensions}

Removal of the mixed waste remaining in the B Plant containment building will be addressed at a later date. The Tri-Party Agreement allows the mixed waste to be left in the containment building through facility deactivation.

If variances, treatment equivalency petitions, or time extensions are required because of delays in the development of treatment, storage, or disposal capacity, they will be applied for in accordance with the procedures detailed in the Tri-Party Agreement or regulations.

\subsection{B PLANT ORGANIC WASTE}

The B Plant organic waste was previously stored in process tanks 27-3 and 28-3 in the B Plant canyon. These tanks are included in the permitted tank treatment and storage TSD unit for organic waste. The facility completed transfer of this waste to a permitted storage TSD unit located outside the facility. This task is part of B Plant deactivation.

\subsubsection{Generation}

The B Plant organic waste is the remaining organic solvents used in the strontium solvent extraction process at the B Plant from 1968 to 1979 (Figure 3-12). This waste has been stored since then. Solvents used in the process included D2EHP, TBP, and NPH. There are no plans to generate more of this waste.

\subsubsection{Characterization}

This section discusses the waste characterization and its basis. The waste designation, the uncertainty of the designation, and the schedule for further characterization also are provided. 
3.13.2.1 Process Knowledge. According to historical process flowsheets, the solvent used in the extraction process consisted of $0.5 \mathrm{M}$ D2EHP, $0.32 \mathrm{M}$ TBP, and NPH diluent. Additional makeup added for solvent losses consisted of $0.58 \mathrm{M} \mathrm{D2EHP,} 0.40 \mathrm{M} \mathrm{TBP}$, and NPH. The latest process knowledge indicates that the waste matrix contains approximately 70 percent $\mathrm{NPH}$, 20 percent D2EHP, and 10 percent TBP.

3.13.2.2 Sample Analyses. Sample analysis of this waste has been attempted at least twice. In February 1989, Tank 27-3 was sampled in an attempt to characterize the waste. Results were inconclusive because the sample included portions of both the aqueous phase and the organic phase and the results were determined to be more indicative of the aqueous portion.

In November 1992, the organic layer in Tank 28-3 was sampled to attempt some preliminary characterization. This analysis showed the presence of the expected constituents, but at concentrations different from expected based on process flowsheets. This deviation was determined to be caused by stratification of the waste. Agitators had not been used to mix the waste and provide a representative sample because of safety concerns related to the long-term storage of the waste in a radioactive environment.

3.13.2.3 Waste Designation and Basis. The waste is designated as F001 through F005 to account for the mixture and derived-from rules related to listed DST and SST waste. Heavy metals (waste codes D004-D011) may also be present, but insufficient sample results are available to confirm this. The storage tanks have been permitted for all these waste codes.

3.13.2.4 Uncertainty of Waste Designation. The designation of the waste with the F001 through F005 waste codes is considered accurate to maintain consistency with RCRA regulations. No data are available to determine the accuracy of the heavy metal waste codes. The designation of WT02 is considered accurate, provided the assumption of the composition of the waste matrix is accurate.

3.13.2.5 Schedule for Further Characterization. An extensive characterization effort is planned for this waste in 1997 as part of the effort to transfer the waste to external permitted storage tanks. This characterization will also be used to support the search for an appropriate final disposition of this waste. The characterization is being conducted in accordance with the sampling and analysis plan (PHMC 1997a).

\subsubsection{Storage}

This section describes the storage units and provides an update of its compliance status.

3.13.3.1 Description of Storage Unit and Capacity. The previous storage TSD unit for the organic waste included 8 tanks with a capacity of 69 cubic meters. All tanks are located in process cells 26 through 30 in the B Plant canyon. All organic waste had been consolidated into Tanks $27-3$ and 28-3, with a combined capacity of 28 cubic meters. These storage tanks can only be accessed remotely using an overhead canyon crane. 
After transfer outside of the B Plant facility, the organic waste is stored in two portable tanks located in a storage area just northeast of B Plant. Each tank has a capacity of 17.5 cubic meters. The tanks were among those used to transport nitric acid from the PUREX Plant to England. All the waste is stored in a single tank; the other tank is available for backup. The tanks are mounted on a concrete slab with secondary containment structures. The storage facility was built to satisfy tank system TSD requirements. This new storage unit has been permitted for storage of organic waste.

3.13.3.2 Amount in Storage. Current estimates are that approximately 17.4 cubic meters of organic waste are stored in tanks. Specific gravity of the waste is estimated to be 0.85 , for a total weight of 15,000 kilograms of waste.

3.13.3.3 Compliance Status. Both the previous storage tanks and the new tanks are properly permitted for storing this waste. The waste was moved to the outside storage tanks in accordance with the Tri-Party Agreement. In addition, alternatives will be explored during the rest of the transition period to identify potential final disposition options for the waste. If the waste is still being stored at B Plant in interim storage and final disposal is not achieved by September 1998, the organic waste will be moved to another RCRA compliant storage location on the Hanford Site.

\subsubsection{Treatment}

The organic waste was treated to reduce the radioactive contamination of the waste. This was done using technology for chemical separation of radionuclides to bring contamination to the desired levels for removing the waste from the B Plant canyon. This effort was conducted in accordance with the Tri-Party Agreement in direct support of Milestones M-82-03 and M-82-08.

Future treatment and disposal options are being examined and will be pursued during the transition period. Some of the options currently being explored include solidification and incineration.

\subsubsection{Waste Reduction}

There are no plans to generate additional quantities of this waste.

\subsubsection{Variances, Treatment Equivalency Petitions, Time Extensions}

The Tri-Party Agreement allows for the storage of this waste at B Plant until September 1998. If final disposition has not occurred by then, the Tri-Party Agreement calls for the waste to be moved to another onsite RCRA storage facility. Treatment options are being explored as part of the facility transition effort. 
I If variance, treatment equivalency petitions, or time extensions are required because of I delays in the development of treatment, storage, or disposal capacity, they will be applied for in I accordance with the procedures detailed in the Tri-Party Agreement or regulations. 


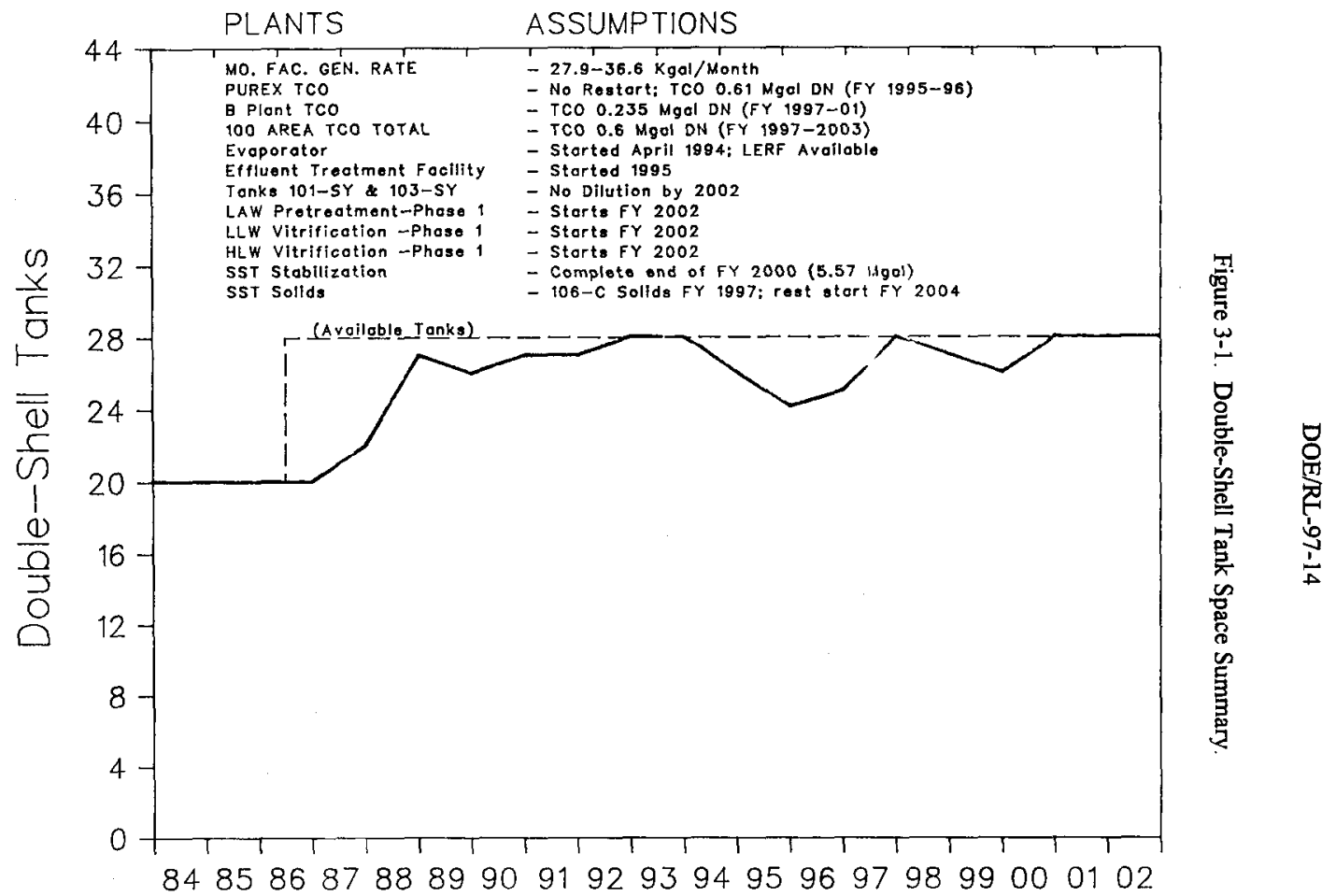

Fiscal Year 


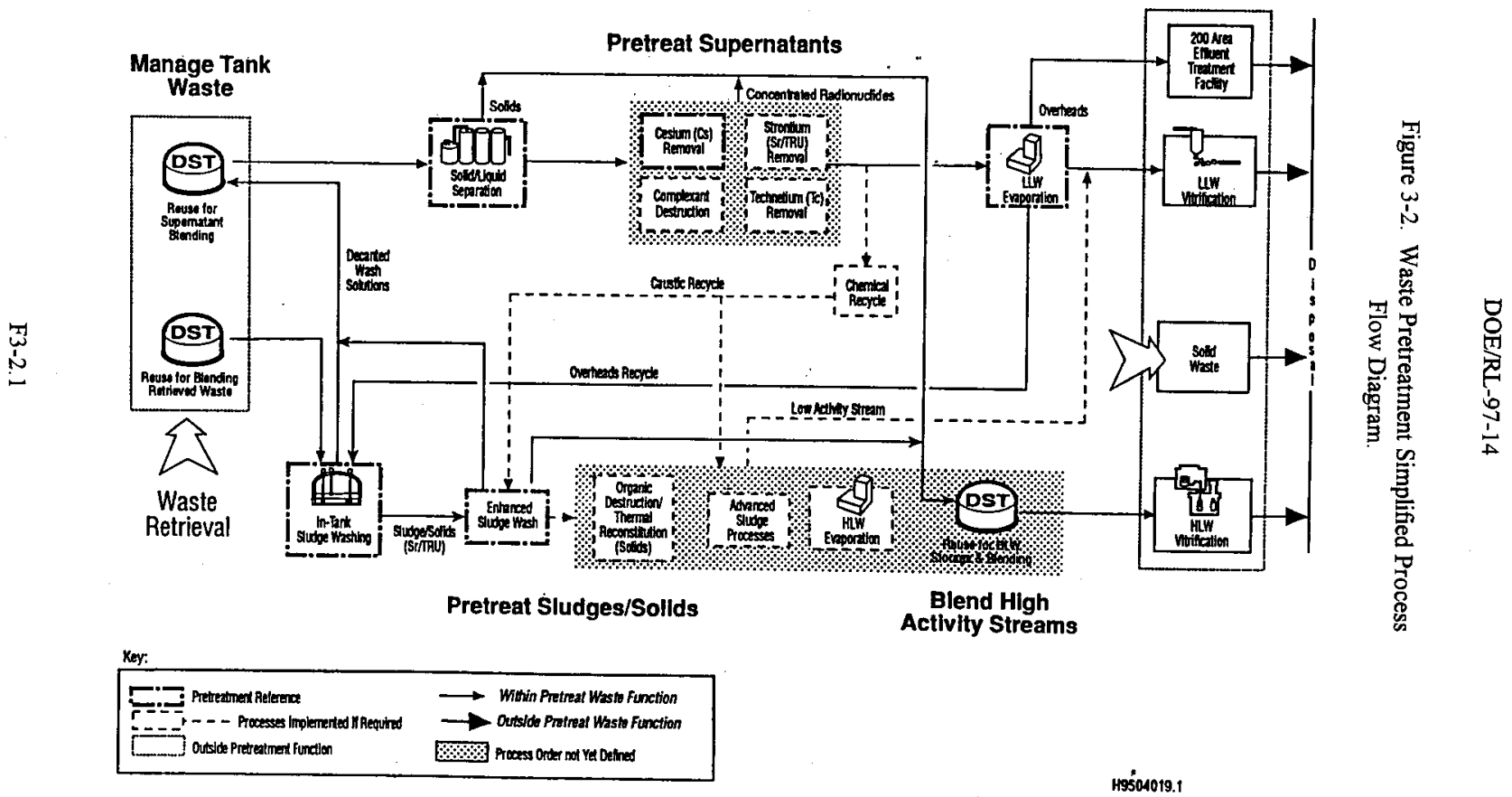


Figure 3-3. Relative Proportions of Supernatant, Sludge, and Salt Cake in Single-Shell Waste.

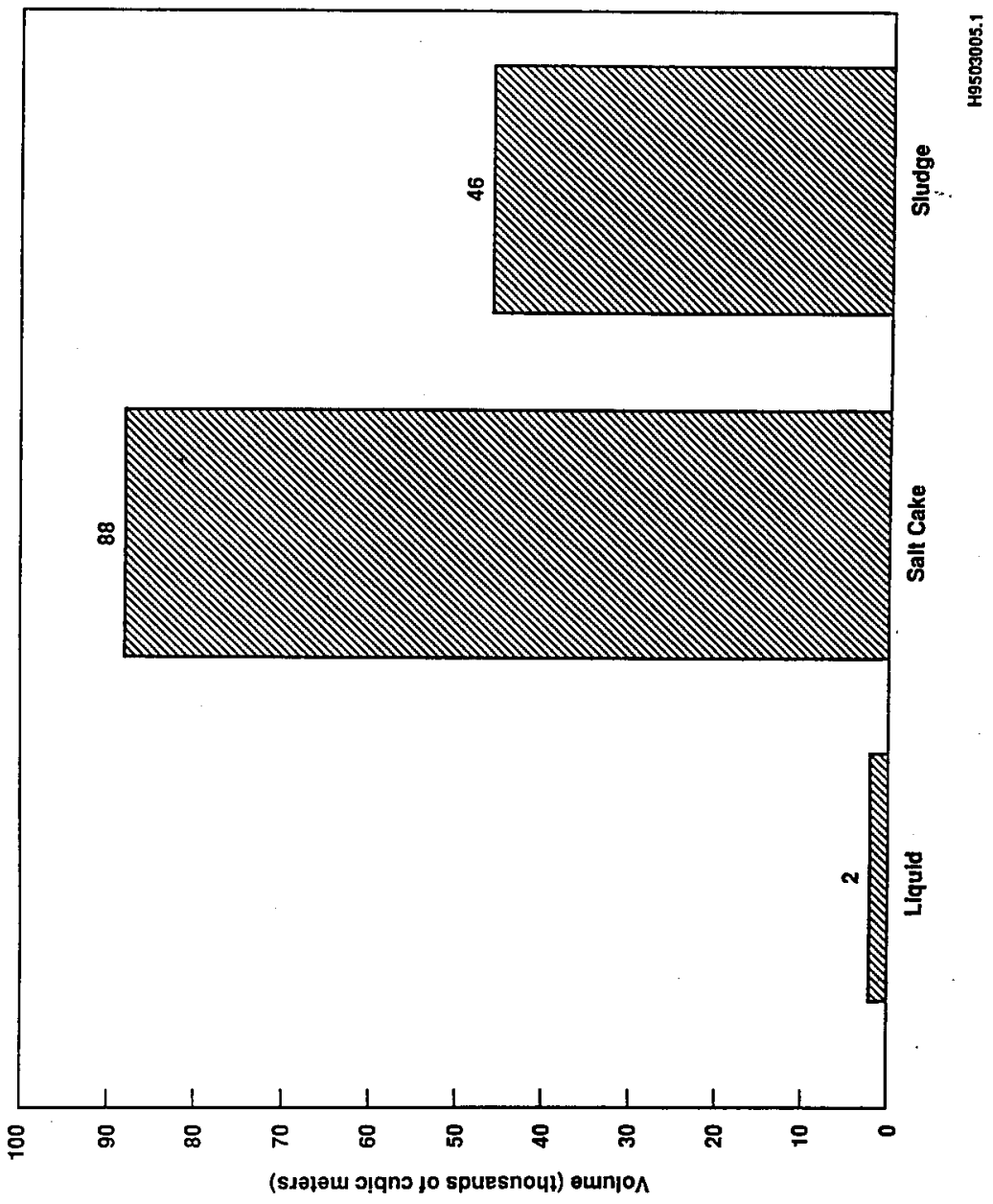




\section{DOE/RL-97-14}

Figure 3-4. Single-Shell Tank Waste Inventory by Tank Farm.

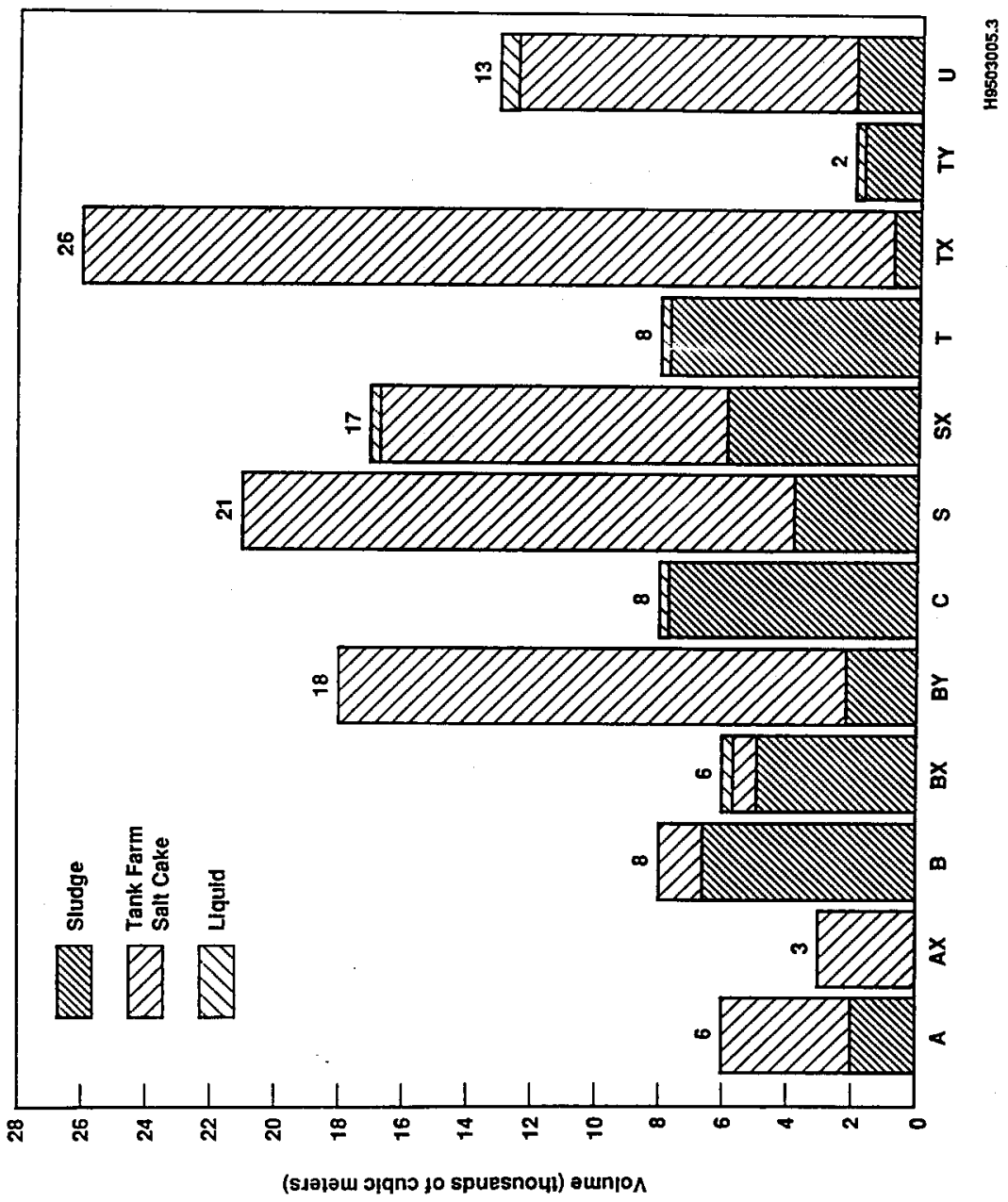


Figure 3-5. Process Flow Diagram for 242-A Evaporator.

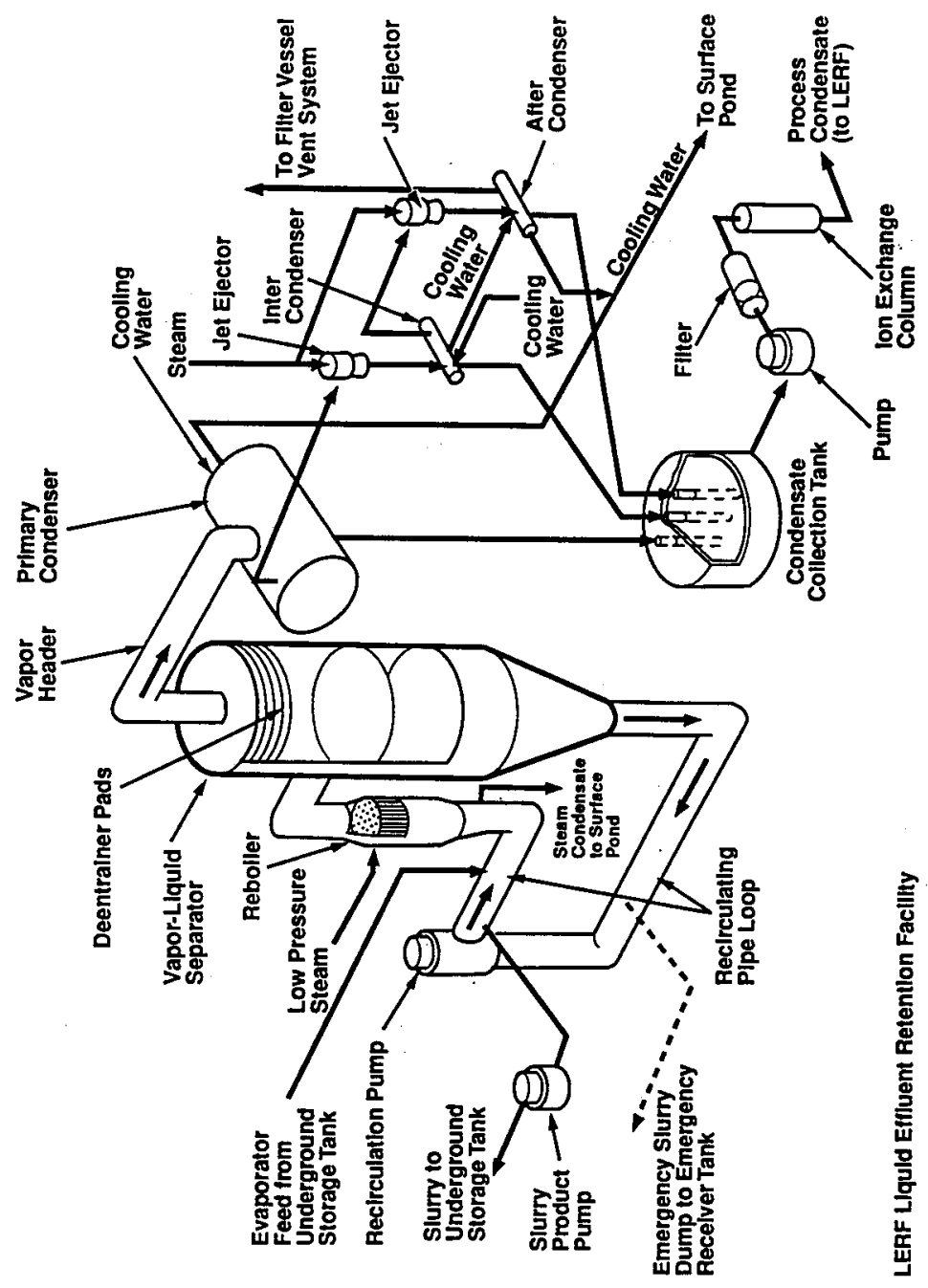


DOE/RL-97-14

Figure 3-6. LERF Basin Design.

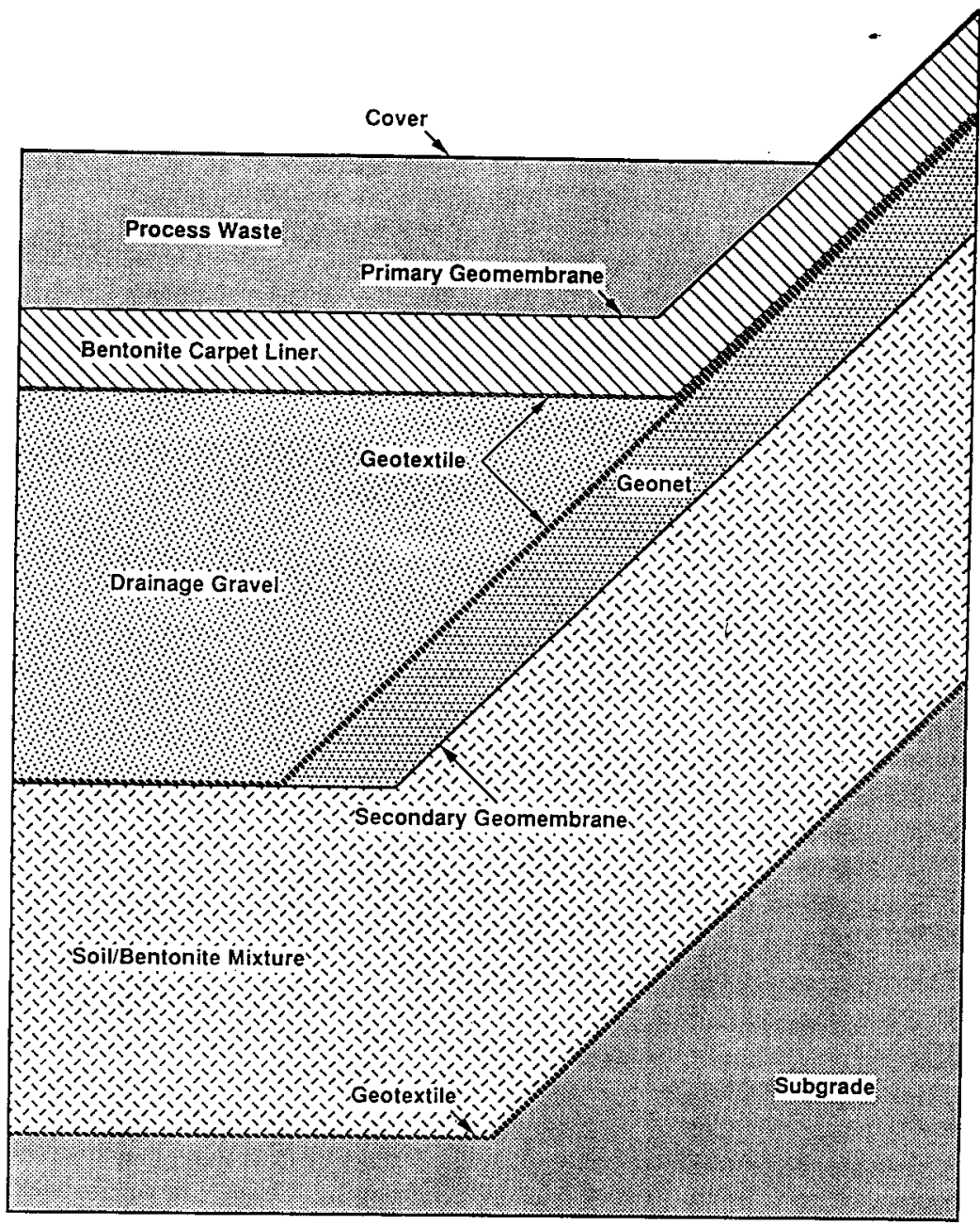

H97010042.1 
DOE/RL-97-14

Figure 3-7. WRAP Module 1, 2336-W.

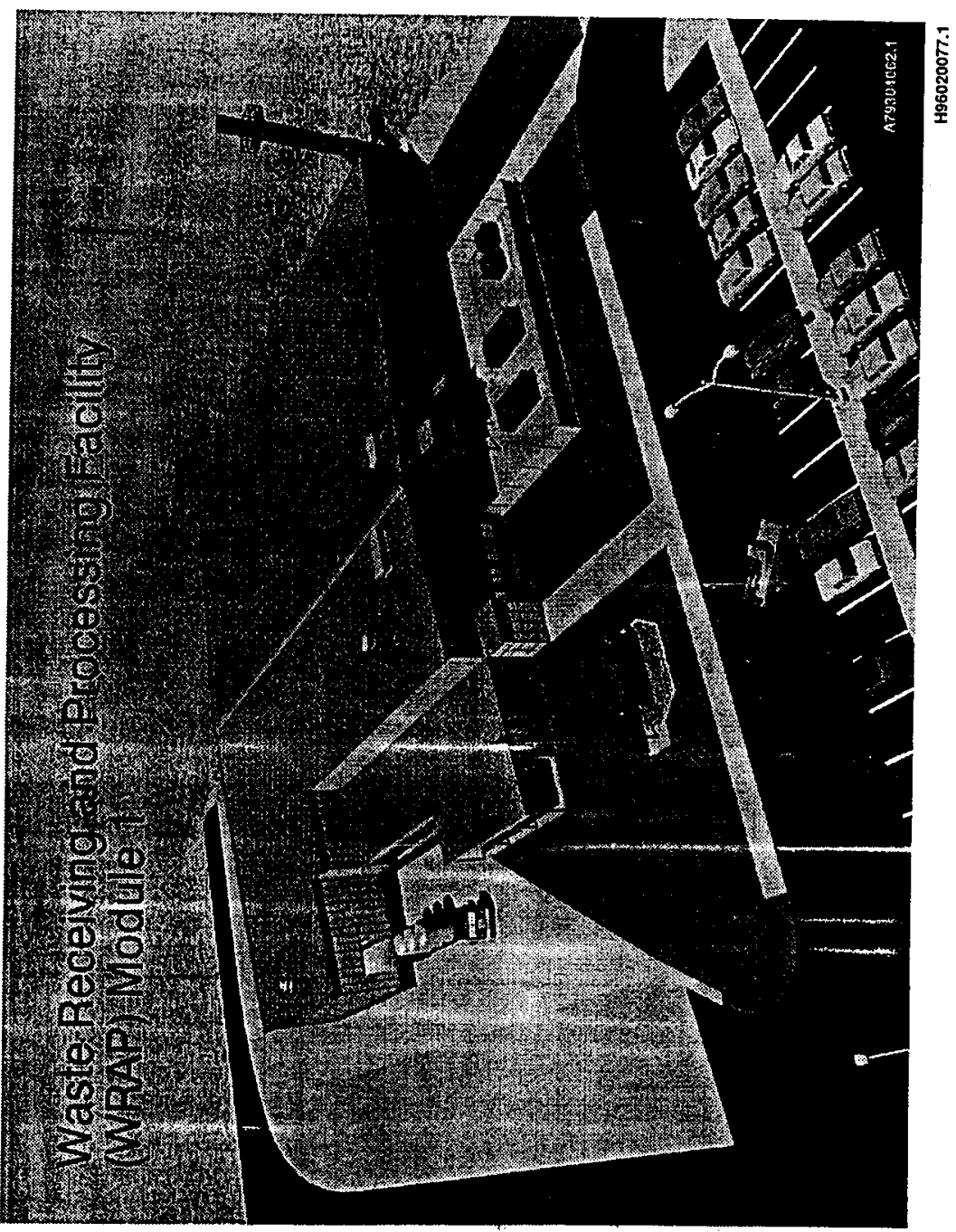


Figure 3-8. Waste Receiving and Processing Module 1 and Commercial Treatment.

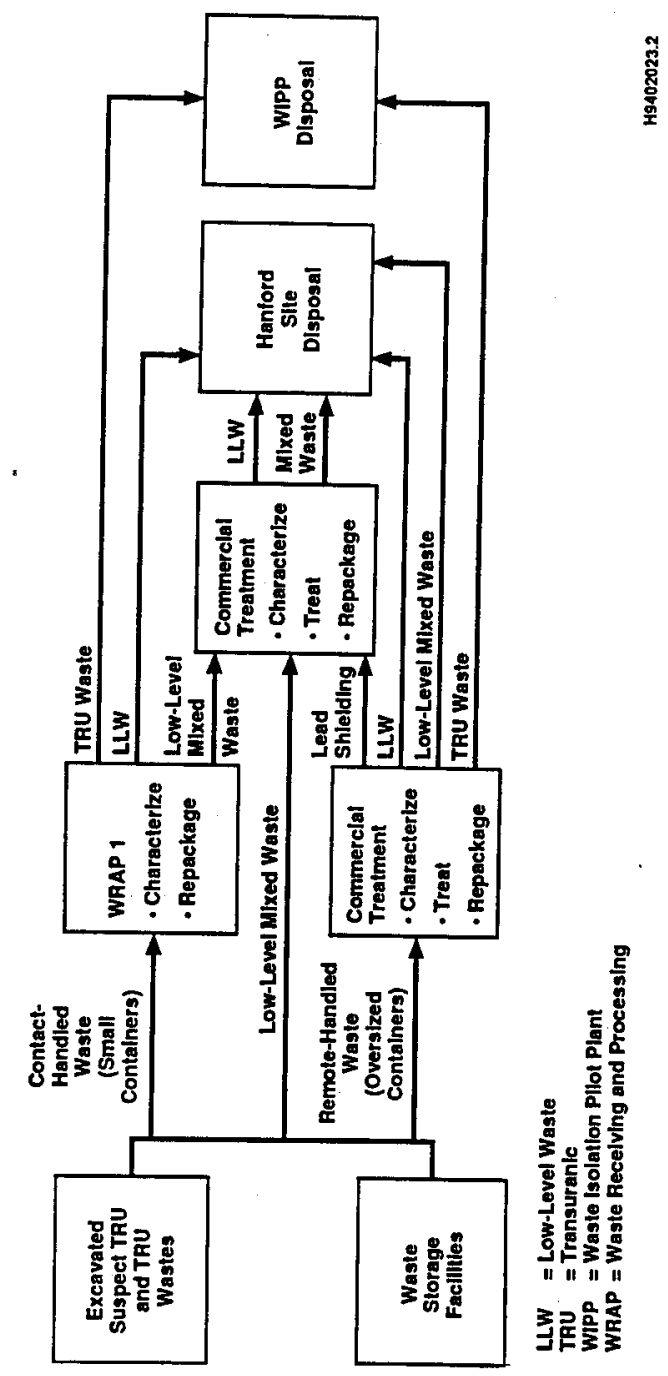

F3-8.1 
Figure 3-9. Typical Configuration of Suspect-TRU and TRU Contact-Handled Waste to be Excavated.

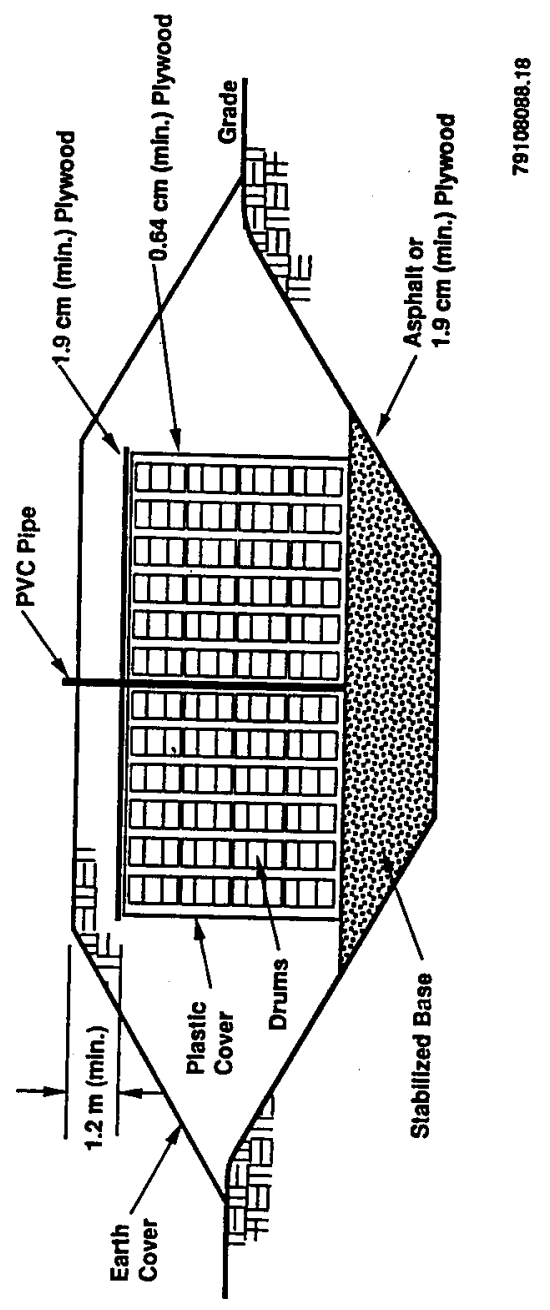


Figure 3-10. Typical Configuration of a Retrievable Storage Unit for Remote-Handled Waste.

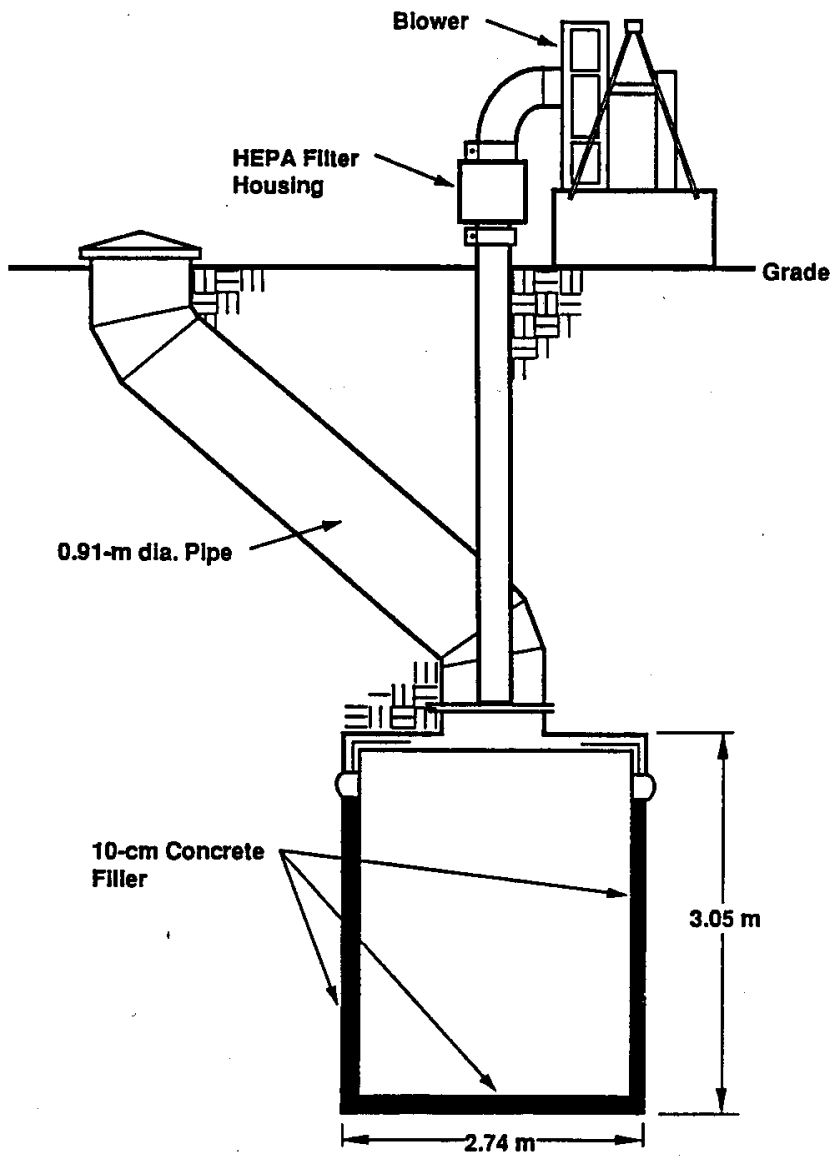

HEPA High-efficiency particulate alr

79108088.19 
Figure 3-11. 224-T Transuranic Storage and Assay Facility Floor Plan.
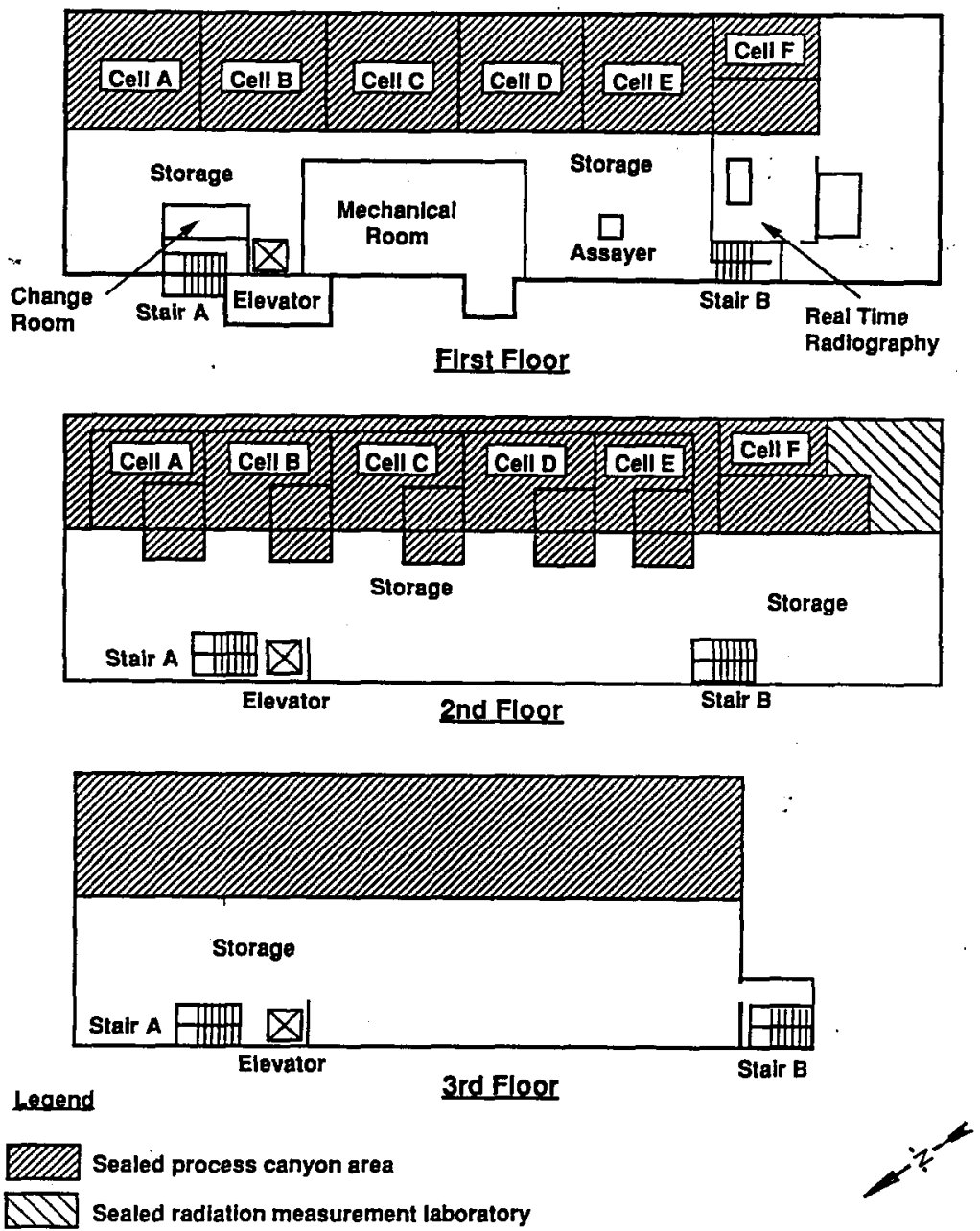

79108088.20 
Figure 3-12. Historical Process Flow Diagram for B Plant Strontium Solvent Extraction Process

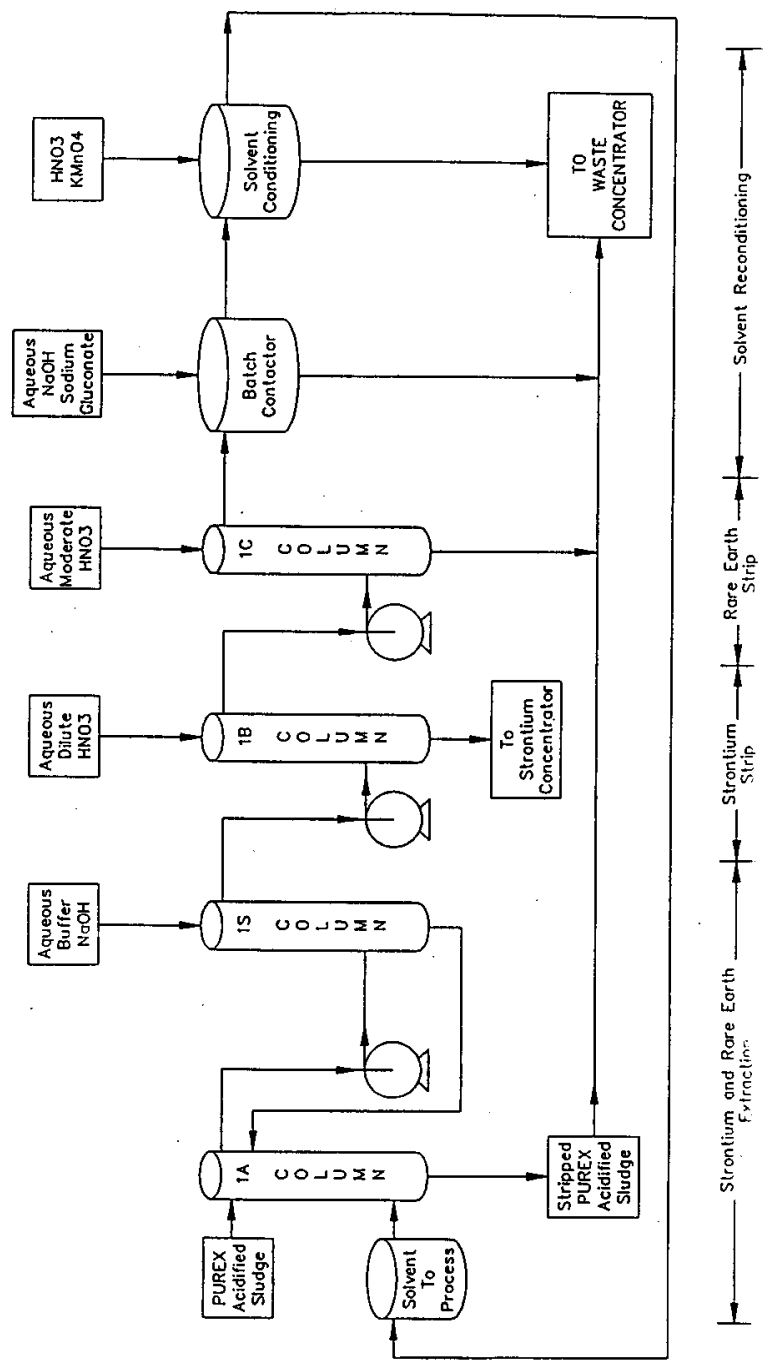


Table 3-1. Estimated Mass of Nonradioactive Chemical Components of Single-Shell and Double-Shell Tank Wastes. (2 sheets)

\begin{tabular}{|c|c|c|c|c|c|c|c|}
\hline \multirow{2}{*}{$\begin{array}{l}\text { Chemical } \\
\text { metric tons }\end{array}$} & \multicolumn{4}{|c|}{ Single-Shell Tanks } & \multicolumn{3}{|c|}{ Double-Shell Tanks } \\
\hline & Sludge & Salt Cake & $\begin{array}{c}\text { Interstitial } \\
\text { Liquid }\end{array}$ & Total & Soluble & Insoluble & Total \\
\hline $\mathrm{Ag}^{+}$ & & & & & $3.28 \mathrm{E}-01$ & $1.38 \mathrm{E}+00$ & $1.70 \mathrm{E}+00$ \\
\hline $\mathrm{Al}(\mathrm{OH})_{4}^{-}$ & $6.25 \mathrm{E}+02$ & $1.25 \mathrm{E}+03$ & $4.57 \mathrm{E}+02$ & $2.33 \mathrm{E}+03$ & $5.09 \mathrm{E}+03$ & & $5.09 \mathrm{E}+0.3$ \\
\hline $\mathrm{Al}^{+3(1)}$ & $1.99 \mathrm{E}+03$ & & & $1.99 \mathrm{E}+03$ & & $6.78 E+01$ & $6.78 \mathrm{E}+01$ \\
\hline $\mathrm{As}^{+3}$ & & & & & $7.70 \mathrm{E}-01$ & $4.98 \mathrm{E}-01$ & $1.27 \mathrm{E}+00$ \\
\hline $\mathrm{B}^{+3}$ & & & & & $5.19 \mathrm{E}-01$ & 9.94 E-0I & $1.51 \mathbf{E}+00$ \\
\hline $\mathrm{Ba}^{+2}$ & & & & & $7.91 \mathrm{E}-01$ & $3.09 \mathrm{E}+00$ & $3.88 \mathrm{E}+00$ \\
\hline $\mathrm{Be}^{+2}$ & & & & & $8.19 \mathrm{E}-02$ & $7.61 \mathrm{E}-03$ & $8.95 \mathrm{E}-02$ \\
\hline $\mathrm{Bi}^{+3}$ & $2.61 \mathrm{E}+02$ & & & $2.61 \mathrm{E}+02$ & $2.26 \mathrm{E}+00$ & & $2.26 \mathrm{E}+00$ \\
\hline $\mathrm{Ca}^{+2}$ & $1.28 \mathrm{E}+02$ & & & $1.28 \mathrm{E}+02$ & $1.03 \mathrm{E}+01$ & $1.15 \mathrm{E}+01$ & $2.18 \mathrm{E}+01$ \\
\hline $\mathrm{Cd}^{+2}$ & $3.84 \mathrm{E}+00$ & & & $3.84 \mathrm{E}+00$ & $1.67 \mathrm{E}-01$ & $6.01 \mathrm{E}+00$ & $6.18 \mathrm{E}+00$ \\
\hline $\mathrm{Ce}^{+3}$ & $2.35 \mathrm{E}+02$ & & & $2.35 \mathrm{E}+02$ & $2.26 \mathrm{E}-02$ & $3.04 \mathrm{E}+00$ & $3.07 \mathrm{E}+00$ \\
\hline $\mathrm{Cl}^{-}$ & $4.00 \mathrm{E}+01$ & & & $4.00 \mathrm{E}+01$ & $2.73 \mathrm{E}+02$ & $1.49 \mathrm{E}+00$ & $2.74 \mathrm{E}+02$ \\
\hline $\mathrm{CO}_{3}^{-2}$ & $1.15 \mathrm{E}+03$ & $4.13 \mathrm{E}+02$ & $3.96 \mathrm{E}+01$ & $1.61 \mathrm{E}+03$ & $1.92 E+03$ & $5.83 \mathrm{E}+01$ & $1.98 \mathrm{E}+03$ \\
\hline $\mathrm{Cr}^{+3}$ & $8.63 \mathrm{E}+01$ & & & $8.63 \mathrm{E}+01$ & & $3.41 \mathrm{E}+01$ & $3.41 \mathrm{E}+01$ \\
\hline $\mathrm{CrO}_{4}^{-2}$ & & & $2.14 \mathrm{E}+01$ & $2.14 \mathrm{E}+01$ & $1.20 \mathrm{E}+02$ & & $1.20 \mathrm{E}+02$ \\
\hline $\mathrm{Cu}^{+2}$ & & & & & $1.77 \mathrm{E}-01$ & $7.46 \mathrm{E}-01$ & $9.23 \mathrm{E}-01$ \\
\hline $\mathrm{F}$ & $8.00 \mathrm{E}+02$ & & $5.00 \mathrm{E}+01$ & $8.05 \mathrm{E}+02$ & $3.52 \mathrm{E}+\mathrm{O} 2$ & $1.91 \mathrm{E}+01$ & $3.71 \mathrm{~L}+02$ \\
\hline $\mathrm{Fe}(\mathrm{CN})_{6}^{-4}$ & $3.22 \mathrm{E}+02$ & & & $3.22 \mathrm{E}+02$ & & & \\
\hline $\mathrm{Fe}^{+3}$ & $6.27 \mathrm{E}+02$ & & & $6.27 \mathrm{E}+02$ & $8.09 \mathrm{E}+00$ & $1.42 \mathrm{E}+02$ & $1.50 \mathrm{E}+02$ \\
\hline $\mathrm{Hg}^{+}$ & $9.00 \mathrm{E}-01$ & & & $9.00 \mathrm{E}-01$ & $5.84 \mathrm{E}-02$ & & $5.84 \mathrm{E}-02$ \\
\hline $\mathrm{K}^{+}$ & & & & & $5.46 \mathrm{E}+02$ & $2.02 \mathrm{E}+01$ & $5.66 \mathrm{E}+02$ \\
\hline $\mathrm{La}^{+}$ & & & & & $2.19 \mathrm{E}-01$ & $2.10 \mathrm{E}+01$ & $2.12 \mathrm{E}+01$ \\
\hline $\mathrm{Li}^{+}$ & & & & & $5.77 \mathrm{E}-03$ & $2.46 \mathrm{E}-02$ & $3.04 \mathrm{E}-(02$ \\
\hline $\mathrm{Mg}^{+2}$ & & & & & $9.65 \mathrm{E}-01$ & $1.10 \mathrm{E}+01$ & $1.20 \mathrm{E}+(11$ \\
\hline $\mathrm{Mn}^{+4}$ & $1.20 \mathrm{E}+02$ & & & $1.20 \mathrm{E}+02$ & $7.69 \mathrm{E}+00$ & $1.80 \mathrm{E}+01$ & $2.57 \mathrm{E}+01$ \\
\hline $\mathrm{Mo}^{+6}$ & & & & & $4.87 \mathrm{E}+00$ & $8,01 \mathrm{E}-01$ & $5.67 \mathrm{E}+00$ \\
\hline $\mathrm{Na}^{+}$ & $1.58 \mathrm{E}+04$ & $3.39 \mathrm{E}+04$ & $2.30 \mathrm{E}+03$ & $5.48 \mathrm{E}+04$ & $1.40 \mathrm{E}+04$ & $2.30 \mathrm{E}+02$ & $1.43 \mathrm{E}+04$ \\
\hline $\mathrm{Ni}^{+2}$ & $1.78 E+02$ & & & $1.78 \mathrm{E}+02$ & $4.07 \mathrm{E}+00$ & $6.57 \mathrm{E}+00$ & $1.06 \mathrm{E}+(0) 1$ \\
\hline
\end{tabular}


Table 3-1. Estimated Mass of Nonradioactive Chemical Components of Single-Shell and Double-Shell Tank Wastes. (2 sheets)

\begin{tabular}{|c|c|c|c|c|c|c|c|}
\hline \multirow{2}{*}{$\begin{array}{l}\text { Chemical } \\
\text { metric tons }\end{array}$} & \multicolumn{4}{|c|}{ Single-Shell Tanks } & \multicolumn{3}{|c|}{ Double-Shell Tanks } \\
\hline & Sludge & Salt Cake & $\begin{array}{c}\text { Interstitial } \\
\text { Liquid }\end{array}$ & Total & Soluble & Insoluble & Total \\
\hline $\mathrm{NO}_{2}^{-}$ & $2.00 \mathrm{E}+03$ & $1.53 \mathrm{E}+03$ & $1.27 \mathrm{E}+03$ & $4.80 \mathrm{E}+03$ & $4.80 \mathrm{E}+03$ & $8.42 \mathrm{E}+00$ & $4.81 \mathrm{E}+03$ \\
\hline $\mathrm{NO}_{3}^{-}$ & $1.48 \mathrm{E}+04$ & $8.03 \mathrm{E}+04$ & $1.71 \mathrm{E}+03$ & $9.68 \mathrm{E}+04$ & $1.03 \mathrm{E}+03$ & $3.91 \mathrm{E}+01$ & $1.03 E+04$ \\
\hline $\mathrm{OH}^{-}$ & $4.22 \mathrm{E}+03$ & $8.51 \mathrm{E}+02$ & $3.15 \mathrm{E}+02$ & $5.39 \mathrm{E}+03$ & $2.33 \mathrm{E}+03$ & $1.23 \mathrm{E}+02$ & $2.45 \mathrm{E}+03$ \\
\hline $\mathrm{Pb}^{+4}$ & & & & & $1.96 \mathbf{E}+00$ & $3.28 \mathrm{E}+00$ & $5.24 \mathrm{E}+00$ \\
\hline $\mathrm{PO}_{4}^{-3}$ & $3.89 \mathrm{E}+03$ & $6.43 \mathrm{E}+02$ & $8.58 \mathrm{E}+01$ & $4.62 \mathrm{E}+03$ & $3.29+E+02$ & $2.16 E+01$ & $3.15 \mathrm{E}+112$ \\
\hline $\mathrm{SiO}_{2}{ }^{-2}$ & $1.21 \mathrm{E}+03$ & & & $1.21 \mathrm{E}+03$ & $1.53 \mathrm{E}+01$ & $2.14 \mathrm{E}+02$ & $2.29 \mathrm{E}+02$ \\
\hline $\mathrm{SO}_{4}^{-2}$ & $5.01 \mathrm{E}+02$ & $1.15 \mathrm{E}+03$ & & $1.65 \mathrm{E}+03$ & $3.86 \mathrm{E}+02$ & $6.68 \mathrm{E}+10$ & $3.93 \mathrm{E}+02$ \\
\hline $\mathrm{Sr}^{+2}$ & $3.60 \mathrm{E}+01$ & & & $3.60 \mathrm{E}+01$ & & & \\
\hline $\operatorname{TOC}^{(2)}$ & & & $2.00 \mathrm{E}+02$ & $2.00 \mathrm{E}+02$ & $1.26 \mathrm{E}+03$ & $6.84 \mathrm{E}+01$ & $1.33 \mathrm{E}+03$ \\
\hline $\mathrm{UO}_{2}^{+2}$ & & & & & $3.54 \mathrm{E}+00$ & $2.68 \mathrm{E}+01$ & $3.03 \mathrm{E}+01$ \\
\hline $\mathrm{V}^{+5}$ & & & & & $6.20 \mathrm{E}-02$ & $1.88 \mathrm{E}-01$ & $2.50 \mathrm{E}-01$ \\
\hline$W^{+4}$ & $1.44 \mathrm{E}+01$ & & & $1.44 \mathrm{E}+01$ & $7.47 \mathrm{E}-01$ & & $7.47 \mathrm{E}-0 \mathrm{I}$ \\
\hline $\mathrm{Zn}^{42}$ & & & & & $3.59 \mathrm{E}+00$ & $9.45 \mathrm{E}-01$ & $4.54 \mathrm{E}+00$ \\
\hline $\mathrm{ZI}^{+4}$ & $2.46 \mathrm{E}+02$ & & & $2.46 \mathrm{E}+02$ & $4.48 \mathrm{E}-01$ & $2.77 \mathrm{E}+02$ & $2.77 \mathrm{I}+02$ \\
\hline $\begin{array}{l}\text { Total w/o } \\
\mathrm{H}_{2} \mathrm{O}\end{array}$ & $4.93 E+04$ & $1.23 \mathrm{E}+05$ & $6.40 \mathrm{E}+04$ & $1.79 E+05$ & $4.18 \mathrm{E}+0.14$ & $1.45 \mathrm{E}+03$ & $4.32 \mathrm{E}+04$ \\
\hline $\mathrm{H}_{2} \mathrm{O}$ & $2.62 \mathrm{E}+04$ & $1.40 \mathrm{E}+04$ & $5.16 \mathrm{E}+03$ & $4.54 \mathrm{E}+04$ & $8.95 \mathrm{E}+04$ & & $8.95 \mathrm{E}+(1) 4$ \\
\hline Total & $7.55 \mathrm{E}+04$ & $1.37 \mathrm{E}+05$ & $1.16 \mathrm{E}+04$ & $2.24 \mathrm{E}+05$ & $1.31 E+05$ & $1.45 E+03$ & $1.33 \mathrm{E}+0.5$ \\
\hline
\end{tabular}

${ }^{(1)} \mathrm{Al}^{+3}$ includes the $\mathrm{Al}$ present in cancrinite and $\mathrm{Al}(\mathrm{OH})_{3}$.

${ }^{(2)}$ TOC includes HEDTA, EDTA, hydroxyacetic acid, citric acid, and other degradation products

Reference: WHC 1995b 
Table 3-2. Waste Generation for Various Facilities and Programs (cubic meters).

\begin{tabular}{|c|c|c|c|c|c|c|c|c|c|c|c|c|}
\hline FY & $\begin{array}{c}\text { B } \\
\text { Plant }\end{array}$ & PUREX & $\begin{array}{c}\text { Tank } \\
\text { Farms }\end{array}$ & $\begin{array}{c}\text { SST to } \\
\text { DST } \\
\text { Pumping }\end{array}$ & $\begin{array}{c}\text { UO, } \\
\text { Plant }\end{array}$ & PFP & $\begin{array}{c}\text { T } \\
\text { Plant }\end{array}$ & $\begin{array}{c}\text { S Plant } \\
\text { (Labora- } \\
\text { tories) }\end{array}$ & $\begin{array}{c}100 \\
\text { Area }\end{array}$ & $\begin{array}{c}300 \\
\text { Area }\end{array}$ & $\begin{array}{c}400 \\
\text { Area }\end{array}$ & Total \\
\hline 1990 & 2,393 & 6,882 & 1,226 & 0 & 0 & 53 & 151 & 121 & 193 & 136 & 0 & 11,155 \\
\hline 1991 & 1,317 & 984 & 776 & 859 & 0 & 0 & 140 & 170 & 0 & 208 & 0 & 4.454 \\
\hline 1992 & 435 & 363 & 155 & 458 & 0 & 136 & 250 & 106 & 0 & 132 & 30 & 2.065 \\
\hline 1993 & 511 & 291 & 144 & 140 & 0 & 19 & 257 & 38 & 0 & 87 & 45 & $1.532^{2}$ \\
\hline 1994 & 53 & 276 & 140 & 836 & 0 & 26 & 76 & 76 & 0 & 110 & 42 & 1.635 \\
\hline 1995 & 129 & 1,154 & 360 & 1,196 & 0 & 0 & 83 & 83 & 0 & 220 & 0 & 3.225 \\
\hline 1996 & 359 & 621 & 500 & 908 & 0 & 15 & 91 & 106 & 0 & 197 & 0 & 2.797 \\
\hline
\end{tabular}

Note: All generation quantities include the volume of any flush water.

In addition to the waste categories in the table, in 1993, approximately 1,336 cubic meters of water was added to DSTs. This water was used to test the upgraded 242-A Evaporator components before restart.

DST $=$ Double-shell tank.

PFP = Plutonium Finishing Plant .

SST $=$ Single-shell tank 
DOE/RL-97-14

Table 3-3. Hanford Site Single-Shell Tank Releases. ${ }^{\text {" }}$ (sheet l of 3)

\begin{tabular}{|c|c|c|}
\hline Tank & Volume $\left(\mathrm{m}^{3}\right)$ & Leak Reported \\
\hline $241-A-103$ & 21 & 1987 \\
\hline 241-A-104 & 9.5 & 1975 \\
\hline 241-A-105 & 170 & 1963 \\
\hline $241-A X-102$ & 11 & 1988 \\
\hline 241-B-104 & 1.5 & 1984 \\
\hline 241-B-107 & 30 & 1980 \\
\hline $241-B-110$ & 38 & 1981 \\
\hline $241-B-112$ & 7.6 & 1978 \\
\hline 241-B-201 & 4.5 & 1980 \\
\hline 241-B-203 & 1.1 & 1983 \\
\hline 241-BX-102 & 265 & 1971 \\
\hline 241-BX-108 & 9.5 & 1974 \\
\hline 241-BY-103 & $<19$ & 1973 \\
\hline 241-BY-107 & 57 & 1984 \\
\hline 241-BY-108 & $<19$ & 1972 \\
\hline $241-C-101$ & 76 & 1980 \\
\hline $241-C-110$ & 7.6 & 1984 \\
\hline 241-C-111 & 21 & 1968 \\
\hline 241-C-201 & 2.1 & 1988 \\
\hline $241-\mathrm{C}-202$ & 1.7 & 1988 \\
\hline 241-C-203 & 1.5 & 1984 \\
\hline 241-C-204 & 1.3 & 1988 \\
\hline $241-S-104$ & 91 & 1968 \\
\hline 241-SX-104 & 23 & 1988 \\
\hline 241-SX-107 & $<19$ & 1964 \\
\hline $241-S X-108$ & 132 & 1962 \\
\hline 241-SX-109 & 38 & 1965,1996 \\
\hline $241-S X-110$ & 21 & 1976 \\
\hline 241-SX-111 & 7.6 & 1974 \\
\hline 241-SX-112 & 114 & 1969 \\
\hline 241-SX-113 & 57 & 1962 \\
\hline
\end{tabular}


Table 3-3. Hanford Site Single-Shell Tank Releases. (sheet 2 of 3)

\begin{tabular}{|c|c|c|}
\hline Tank & Volume $\left(\mathrm{m}^{3}\right)$ & Leak Reported \\
\hline 241-SX-115 & 189 & 1965 \\
\hline $241-\mathrm{T}-101$ & 28 & 1992 \\
\hline $241-T-103$ & $<3.8$ & 1974 \\
\hline 241-T-106 & 436 & 1973 \\
\hline $241-T-108$ & $<3.8$ & 1974 \\
\hline 241-T-111 & $<3.8$ & 1984,1994 \\
\hline $241-\mathrm{T}-107$ & 9.5 & 1984 \\
\hline 241-T-109 & $<3.8$ & 1974 \\
\hline 241-TX-107 & 9.5 & 1984 \\
\hline 241-TY-101 & $<3.8$ & 1973 \\
\hline 241-TY-103 & 11 & 1973 \\
\hline 241-TY-104 & 5.3 & 1981 \\
\hline 241-TY-105 & 133 & 1960 \\
\hline 241-TY-106 & 76 & 1959 \\
\hline 241-U-101 & 114 & 1959 \\
\hline 241-U-104 & 208 & 1961 \\
\hline 241-U-110 & 31 & 1975 \\
\hline $241-U-112$ & 32 & 1980 \\
\hline $241-\mathrm{AX}-104^{\mathrm{b}}$ & -- & 1977 \\
\hline 241-B-101 ${ }^{\mathrm{b}}$ & -- & 1974 \\
\hline 241-B-103 & -- & 1978 \\
\hline 241-B-105 & -- & 1978 \\
\hline 241-B-111 $1^{b}$ & - & 1978 \\
\hline $241-\mathrm{BX}-101^{\mathrm{b}}$ & -- & 1972 \\
\hline $241-B X-110^{b}$ & - & 1976 \\
\hline $241-\mathrm{BX}-111^{\mathrm{b}}$ & -- & 1984,1993 \\
\hline 241-BY-105 & -- & 1984 \\
\hline 241-BY-106 & -- & 1984 \\
\hline $241-\mathrm{SX}-114^{\mathrm{b}}$ & -- & 1972 \\
\hline
\end{tabular}


Table 3-3. Hanford Site Single-Shell Tank Releases. (sheet 3 of 3)

\begin{tabular}{|l|c|c|}
\hline \multicolumn{1}{|c|}{ Tank } & Volume $\left(\mathrm{m}^{3}\right)$ & Leak Reported \\
\hline $241-\mathrm{TX}-105^{\mathrm{b}}$ & -- & 1977 \\
\hline $241-\mathrm{TX}-110^{\mathrm{b}}$ & -- & 1977 \\
\hline $241-\mathrm{TX}-113^{\mathrm{b}}$ & -- & 1974 \\
\hline $241-\mathrm{TX}-114^{\mathrm{b}}$ & -- & 1974 \\
\hline $241-\mathrm{TX}-115^{\mathrm{b}}$ & -- & 1977 \\
\hline $241-\mathrm{TX}-116^{\mathrm{b}}$ & -- & 1977 \\
\hline $241-\mathrm{TX}-117^{\mathrm{b}}$ & -- & 1977 \\
\hline Total estimated leakage volume from 67 tanks: $3,148 \mathrm{~m}^{3}$. \\
\hline
\end{tabular}

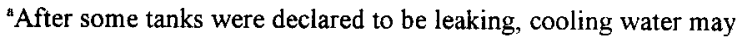
have been added to aid evaporative cooling. It is believed that some of this water did not evaporate and, therefore, went into the ground. As of October 1990, estimates ranged from 190 to 3,000 cubic meters. The past practice was to exclude the cooling water from the leak volume estimate. The volumes provided and date of initial release are the subject of continued evaluation and refinement and may be revised for improved accuracy as a result of these evaluations. In addition, documents show that from 1946 to $1966,456,725$ cubic meters $(120,661,000$ gallons) of liquid wastes were intentionally discharged from SSTs at the Hanford Site directly to the ground on the 200 Area plateau (WHC 1991c). The majority of this waste was discharged from 1946 to 1958 as a result of the early plutonium and uranium recovery processes conducted in the 221-B Facility (B Plant), 221-T Facility (T Plant), and the 221-U Facility (U Plant). In addition, from 1960 to 1966 laboratory wastes from the 300 Area and equipment decontamination wastes from the 200 West Area were routed through SSTs before discharge to the ground. No wastes have been discharged intentionally to the ground from SSTs since 1966, and no wastes have ever been discharged directly to the ground from the newer DSTs located at the Hanford Site.

'Individual release volumes for these tanks have not been determined. The total volume release from these tanks is estimated to be 570 cubic meters.

$$
\text { SST }=\text { Single-shell tank }
$$


Table 3-4. Plutonium-Uranium Extraction Plant Storage Inventories. (sheet 1 of 2)

Plutonium-Uranium Extraction Plant Tunnels Inventory.

\begin{tabular}{|c|c|c|c|c|c|c|c|c|}
\hline $\begin{array}{c}\text { Date } \\
\text { transferred } \\
\text { to tunnel }\end{array}$ & $\begin{array}{l}\text { Tunnel } \\
\text { number }\end{array}$ & $\begin{array}{c}\text { Mass (kg) } \\
\text { of lead } \\
\text { transferred }\end{array}$ & $\begin{array}{l}\text { Mass (kg) } \\
\text { of mercury } \\
\text { transferred }\end{array}$ & $\begin{array}{c}\text { Mass }(\mathrm{kg}) \\
\text { of silver } \\
\text { nitrate } \\
\text { transferred }\end{array}$ & $\begin{array}{c}\text { Mass (kg) } \\
\text { of } \\
\text { cadmium } \\
\text { transferred }\end{array}$ & $\begin{array}{c}\text { Mass }(\mathrm{kg}) \\
\text { of } \\
\text { chromium } \\
\text { transferred }\end{array}$ & $\begin{array}{c}\text { Mass (kg) } \\
\text { of mineral } \\
\text { oil } \\
\text { transferred }\end{array}$ & $\begin{array}{c}\text { Mass }(\mathrm{kg}) \\
\text { of barium } \\
\text { transferred }\end{array}$ \\
\hline $06-60$ & 1 & 115 & $\cdots$ & -- & - & -- & & \\
\hline $12-24-60$ & 1 & 115 & -- & -- & - & - & & \\
\hline $2-26-71$ & 2 & -- & -- & 625 & - & - & & \\
\hline $12-22-71$ & 2 & $=$ & 45 & - & - & -- & & \\
\hline $09-30-72$ & 2 & -- & 45 & - & - & - & & \\
\hline $01-18-86$ & 2 & -- & 40 & - & 43 & - & & \\
\hline $11-18-87$ & 2 & 2,540 & -- & - & -- & - & & \\
\hline $5-13-88$ & 2 & 230 & - & 115 & 13 & -- & & \\
\hline $01-27-95$ & 2 & - & -- & -- & -- & 8 & & \\
\hline $02-08-95$ & 2 & 1,930 & - & -- & -- & -- & & \\
\hline $3-11-96$ & 2 & 3,232 & & & 2 & & & \\
\hline $4-26-96$ & 2 & 1,802 & & & 10.5 & 1.0 & 8.5 & 3 \\
\hline $6-12-96$ & 2 & 0.001 & & & 0.001 & 0.002 & & 0.004 \\
\hline Total & N/A & 9,964 & 130 & 740 & 68.5 & 9 & 8.5 & 3 \\
\hline
\end{tabular}

Note: $9,964 \mathrm{~kg}$ of lead has a volume of $0.89 \mathrm{~m}^{3}$.

$130 \mathrm{~kg}$ of mercury has a volume of about $0.01 \mathrm{~m}^{3}$ at $23^{\circ} \mathrm{C}$.

$740 \mathrm{~kg}$ of silver nitrate has a volume of $0.07 \mathrm{~m}^{3}$.

$68.5 \mathrm{~kg}$ of cadmium have a volume of $7.88 \times 10^{-3} \mathrm{~m}^{3}$.

$9 \mathrm{~kg}$ of chromium have a volume of $1.26 \times 10^{-3} \mathrm{~m}^{3}$.

$3 \mathrm{~kg}$ of barium has a volume of $8.29 \times 10^{-4} \mathrm{~m}^{3}$.

$8.5 \mathrm{~kg}$ of mineral oil has a volume of $7.4 \times 10^{-3} \mathrm{~m}^{3}$.

$\mathrm{N} / \mathrm{A}=$ Not applicable 
| Table 3-4. Plutonium-Uranium Extraction

Plant Storage Inventories. (sheet 2 of 2)

Plutonium-Uranium Extraction Plant

Containment Building Storage Inventory.

\begin{tabular}{|c|c|}
\hline Date transferred & Chromium Mass (kg) \\
\hline $4-29-92$ & 1.0 \\
\hline Total & 1.0 \\
\hline
\end{tabular}

Note: $1.0 \mathrm{~kg}$ of chromium has a volume of $1.4 \times 10^{-4} \mathrm{~m}^{3}$. 
Table 3-5. Forecasted Low-Level and Transuranic Waste to be Stored at the Central Waste Complex.

\begin{tabular}{|c|c|}
\hline Year & Total (cubic meters) \\
\hline 1997 & 1,220 \\
\hline 1998 & 1,220 \\
\hline 1999 & 1,340 \\
\hline 2000 & 1,630 \\
\hline 2001 & 2,020 \\
\hline 2002 & 2,160 \\
\hline 2003 & 1,490 \\
\hline 2004 & 1,570 \\
\hline 2005 & 2,000 \\
\hline 2006 & 1,580 \\
\hline 2007 to 2070 & 47,410 \\
\hline
\end{tabular}


Table 3-6. Solid Waste Treatment Matrix. (3 sheets)

\begin{tabular}{|c|c|c|c|c|c|c|c|}
\hline $\begin{array}{l}\text { Waste Streams/ } \\
\text { Categories }\end{array}$ & $\begin{array}{l}\text { Non-thermal } \\
\text { Stabilization } \\
\text { Treatment (onsite } \\
\text { and/or offsite } \\
\text { commercial contract) }\end{array}$ & $\begin{array}{l}\text { Thermal } \\
\text { Treatment (offsite } \\
\text { commercial } \\
\text { contracts) }\end{array}$ & $\begin{array}{l}\text { Macroencapsulation } \\
\text { Treatment (onsite and/or } \\
\text { offsite commercial } \\
\text { contract) }\end{array}$ & $\begin{array}{l}\text { Decontamination } \\
\text { (onsite at T Plant) }\end{array}$ & $\begin{array}{l}\text { WRAP-1 } \\
\text { (Onsite) }\end{array}$ & $\begin{array}{l}\text { Direct Disposal } \\
\text { (No Treatment } \\
\text { Required) }\end{array}$ & $\begin{array}{r}\text { Milestones } \\
\text { M33 \& M91 }\end{array}$ \\
\hline \multicolumn{8}{|c|}{ LLMW } \\
\hline $\begin{array}{l}\text { 183-H Basin } \\
\text { solidified liquids }\end{array}$ & & & & & & $\begin{array}{l}\text { Yes (defaults to } \\
\text { non-thermal } \\
\text { treatment if not } \\
\text { successful) }\end{array}$ & \\
\hline $\begin{array}{l}\text { 183-H Basin } \\
\text { crystalline solids }^{1}\end{array}$ & Yes & & & & & & \\
\hline $\begin{array}{l}\text { 183-H Basin } \\
\text { sludge }^{1}\end{array}$ & Yes & & & & & & \\
\hline $\begin{array}{l}\text { 183-H Basin } \\
\text { debris waste }\end{array}$ & Yes & . & Yes & & & & \\
\hline $\begin{array}{l}\text { Non-thermally } \\
\text { treatable } \\
\text { particulate, sludge, } \\
\text { and soil waste }\end{array}$ & Yes & & & & & & \\
\hline $\begin{array}{l}\text { Tank farms' } \\
\text { backlog soils }\end{array}$ & & & & & & $\begin{array}{l}\text { Yes (defaults to } \\
\text { thermal treatment } \\
\text { if not successful }\end{array}$ & \\
\hline $\begin{array}{l}\text { Tank farms } \\
\text { backlog debris }\end{array}$ & & & Yes & & & & \\
\hline $\begin{array}{l}\text { Generic debris } \\
\text { waste }\end{array}$ & & & Yes & & & & \\
\hline $\begin{array}{l}\text { Non-thermally } \\
\text { treatable labpacks }\end{array}$ & Yes & & & & Yes & & \\
\hline
\end{tabular}


Table 3-6. Solid Waste Treatment Matrix. (3 sheets)

\begin{tabular}{|c|c|c|c|c|c|c|c|}
\hline $\begin{array}{l}\text { Waste Streams/ } \\
\text { Categonies }\end{array}$ & $\begin{array}{c}\text { Non-thermal } \\
\text { Stabilization } \\
\text { Treatment (onsite } \\
\text { and/or offsite } \\
\text { commercial contract) }\end{array}$ & $\begin{array}{l}\text { Thermal } \\
\text { Treatment (offsite } \\
\text { commercial } \\
\text { contracts) }\end{array}$ & $\begin{array}{l}\text { Macroencapsulation } \\
\text { Treatment (onsite and/or } \\
\text { offsite commercial } \\
\text { contract) }\end{array}$ & $\begin{array}{l}\text { Decontamination } \\
\text { (onsite at T Plant) }\end{array}$ & $\begin{array}{l}\text { WRAP-1 } \\
\text { (Onsite) }\end{array}$ & $\begin{array}{l}\text { Direct Disposal } \\
\text { (No Treatment } \\
\text { Required) }\end{array}$ & $\begin{array}{c}\text { Milestones } \\
\text { M33 \& M91 }\end{array}$ \\
\hline $\begin{array}{l}\text { Thermally } \\
\text { treatable labpacks }\end{array}$ & & Yes & & & & & \\
\hline Elemental mercury & & & & & Yes & & \\
\hline Elemental lead & & & Yes & Yes & & & \\
\hline $\begin{array}{l}\text { Mercury solutions, } \\
\text { particulate and } \\
\text { sludges } \\
(2260 \mathrm{ppm} \mathrm{Hg})^{2}\end{array}$ & & Yes (retorting) & & & & & \\
\hline $\begin{array}{l}\text { Cadmium- and } \\
\text { lead-containing } \\
\text { batteries }^{3}\end{array}$ & & $\begin{array}{l}\text { Yes (metal } \\
\text { recovery) }\end{array}$ & & & & & \\
\hline $\begin{array}{l}\text { RH-LLMW (all } \\
\text { types) }\end{array}$ & & & & & & & Yes \\
\hline \multicolumn{8}{|c|}{ TRU/TRUM } \\
\hline CH-TRU/TRUM & & & & & Yes & & \\
\hline \multicolumn{8}{|c|}{ LLW } \\
\hline CH-LLW & & & & & $\begin{array}{c}\text { Yes } \\
\text { (verifica- } \\
\text { tion only) }\end{array}$ & Yes & \\
\hline
\end{tabular}


Table 3-6. Solid Waste Treatment Matrix. (3 sheets)

\begin{tabular}{|l|c|c|c|c|c|c|}
\hline $\begin{array}{c}\text { Waste Streams/ } \\
\text { Categories }\end{array}$ & $\begin{array}{c}\text { Non-thermal } \\
\text { Stabilization } \\
\text { Treatment (onsite } \\
\text { and/or offsite } \\
\text { commercial contract) }\end{array}$ & $\begin{array}{c}\text { Thermal } \\
\text { Treatment (offsite } \\
\text { commercial } \\
\text { contracts) }\end{array}$ & $\begin{array}{c}\text { Macroencapsulation } \\
\text { Treatment (onsite and/or } \\
\text { offsite commercial } \\
\text { contract) }\end{array}$ & $\begin{array}{c}\text { Decontamination } \\
\text { (onsite at T Plant) }\end{array}$ & $\begin{array}{c}\text { WRAP-1 } \\
\text { (Onsite) }\end{array}$ & $\begin{array}{c}\text { Direct Disposal } \\
\text { (No Treatment } \\
\text { Required) }\end{array}$ \\
\hline RH-LLW & & $\begin{array}{c}\text { Milestones } \\
\text { M33 \& M91 }\end{array}$ & $\begin{array}{c}\text { Yes } \\
\text { (verification } \\
\text { only) }\end{array}$ \\
\hline
\end{tabular}

'The LDR administrators have approved use of stabilization treatment in lieu of the specified combustion treatment for formic acid (U123).

${ }^{2} \mathrm{High}$-mercury subcategory waste (i.e., $\left.2260 \mathrm{mg} / \mathrm{kg} \mathrm{Hg}\right)\left(<1 \mathrm{~m}^{3}\right)$ currently in storage and minimal additional quantity forecasted. The subject waste will be stored on the Hanford Site until either commercial or DOE site capacity is available.

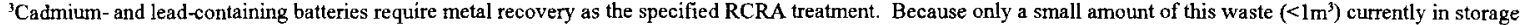
and minimal additional quantity forecasted. he subject waste will be stored on the Hanford Site until either cornmercial or DOE site capacity is available. 


\subsection{REFERENCES}

\subsection{APPLICABLE DOCUMENTS}

Adams, M. R., L. Jensen, and W. W. Schulz, 1986, Preliminary Assessment of the TRAC Model Predictor of Key Radionuclides, RHO-RE-EV-89-P, Rockwell Hanford Operations, Richland, Washington.

Babad, H., R. J. Cash, S. J. Eberlein, G. D. Johnson, J. E. Meacham, J. W. Osborne, M. A. Payne, and D. A. Turner, 1995, Progress in Resolving Hanford Site High-Level Waste Tank Safety Issues, WHC-SA-2369-FP, Westinghouse Hanford Company, Richland, Washington.

DOE, 1987, Final Environmental Impact Statement - Disposal of Hanford Defense High-Level, Transuranic and Tank Wastes, DOE/EIS-0013, Vol. 1, U.S. Department of Energy, Washington, D.C.

DOE, 1990, National Report on Prohibited Wastes and Treatment Options, U.S. Department of Energy, Washington, D.C.

DOE, 1994, Anmual Report of Waste Generation and Pollution Prevention Progress 1994, DOE/EM-0310, U.S. Department of Energy, Headquarters, Washington, D.C.

Ecology, EPA, and DOE, 1989, Hanford Federal Facility Agreement and Consent Order, as amended, Washington State Department of Ecology, U.S. Environmental Protection Agency, and U.S. Department of Energy, Olympia, Washington.

EPA, 1986, Test Methods for the Evaluation of Solid Waste: Physical/Chemical Methods, SW-846, 3rd ed., U.S. Environmental Protection Agency, Washington, D.C.

PHMC, 1997a, Organic Phase and Aqueous Phase Sampling Analysis Plan, HNF-SD-WM-EV-108, Rev. 1, B \& W Hanford Company, Richland, Washington.

PHMC, 1997b, Waste Tank Summary Report for Month Ending December 31, 1996, HNF-EP-0182-105, Lockheed Martin Hanford Company, Richland, Washington.

PNNL, 1995, 324 Building REC and HLV Tank Closure Plan, PNL-10890, Pacific Northwest National Laboratory, Richland, Washington.

RHO, 1985, Hanford Defense Waste Disposal Alternatives: Engineering Support Data for the Hanford Defense Waste Environmental Impact Statement, RHO-RE-ST-30P, Rockwell Hanford Operations, Richland, Washington. 
RL, 1989a, Hanford Site Dangerous Waste Part A Permit Application, DOE/RL-88-21, Vol. 1-3,

U.S. Department of Energy, Richland Operations Office, Richland, Washington.

RL, 1990b, PUREX Storage Tunnels Dangerous Waste Permit Application, DOE/RL-90-24,

Rev. 2, U.S. Department of Energy, Richland Operations Office, Richland, Washington.

RL, 1991a, Double-Shell Tank System Dangerous Waste Permit Application, DOE/RL-90-39,

U.S. Department of Energy, Richland Operations Office, Richland, Washington.

I

RL, 1994a, Tank Waste Remediation System Integrated Technology Plan, DOE/RL-92-61,

Rev. 2 (February 28, 1994), U.S. Department of Energy, Richland Operations Office,

Richland, Washington.

RL, 1995a, Direct Disposal Team Report-Options for Treating and Disposing of Current and Future Mixed Waste Streams on the Hanford Facility, DOE/RL-95-35, Rev. 0,

U.S. Department of Energy, Richland Operations Office, Richland, Washington.

RL, 1995c, Single-Shell Tank Closure Work Plan, DOE/RL-89-16, Rev. A, U.S. Department of Energy, Richland Operations Office, Richland, Washington.

Sasaki, L. M., 1990, Waste Characterization Plan for the Hanford Site Single-Shell Tanks, WHC-EP-0210, REV. 2, Westinghouse Hanford Company, Richland, Washington

Weiss, R. L., 1986, TY Tank Farm Waste Characterization Data, RHO-WM-TI-1P, Rockwell Hanford Operations, Richland, Washington.

WHC, 1989a, Characterization Plan for Stored Contact Handled Transuranic Waste, WHC-EP-0223, Westinghouse Hanford Company, Richland, Washington.

WHC, 1989b, Sampling Plan for Retrievably Stored Contact Handled Transuranic Waste at Hanford, WHC-EP-0226, Westinghouse Hanford Company, Richland, Washington.

WHC, 1990c, Functional Requirements Baseline for the Closure of Single-Shell Tanks-Draft, WHC-EP-0338, Westinghouse Hanford Company, Richland, Washington.

WHC, 1990d, Hanford Site LDR RMW Stream Data Package, WHC-MR-0224, Westinghouse Hanford Company, Richland, Washington.

WHC, 1990e, A History of the 200 Area Tank Farms, WHC-MR-0132, Westinghouse Hanford Company, Richland, Washington.

WHC, 1990h, Single-Shell Tank Waste Retrieval Study, WHC-EP-0352, Westinghouse Hanford Company, Richland, Washington. 
WHC, 1990i, Solid Waste Management Program Plan, WHC-EP-0363, Rev 1, Westinghouse Hanford Company, Richland, Washington.

WHC, 1991c, Tank Wastes Discharged Directly to Soil at the Hanford Site, WHC-MR-0227, Westinghouse Hanford Company, Richland, Washington.

WHC, 1991f, 1991 Borehole Completion Package for the Low-Level Burial Grounds, WHC-SD-EN-DP-044, Rev. 0, Westinghouse Hanford Company, Richland, Washington.

WHC, 1992c, Tank Waste Technical Options Report, WHC-EP-0616, Westinghouse Hanford Company, Richland, Washington.

WHC, 1992d, 1992 Borehole Completion Package for the Low-Level Burial Grounds, WHC-SD-EN-DP-049, Rev. 0, Westinghouse Hanford Company, Richland, Washington.

WHC, 1993a, Alternative Pretreatment Technologies for Removal of Transuranic Elements from Selected Hanford Site Wastes, WHC-EP-0577, Westinghouse Hanford Company, Richland, Washington.

WHC, 1993d, Anmual Report of Tank Waste Treatability, WHC-EP-0365-03, Westinghouse Hanford Company, Richland, Washington.

I WHC, 1993e, Hanford Site Solid Waste Acceptance Criteria, WHC-EP-0063-4, as updated by page changes May 1996, latest, Westinghouse Hanford Company, Richland, Washington.

WHC, 1994a, Tank Waste Remediation System Facility Configuration Study, WHC-SD-WM-ES-295, Westinghouse Hanford Company, Richland, Washington.

WHC, 1994b, TWRS Process Flowsheet, WHC-SD-WM-TI-613, Rev. 1, Westinghouse Hanford Company, Richland, Washington.

WHC, 1994c, 1993 Borehole Completion Data Package for the Low-Level Burial Grounds, WHC-SD-EN-DP-086, Rev. 0, Westinghouse Hanford Company, Richland, Washington.

WHC, 1995b, Single-Shell and Double-Shell Tank Waste Inventory Data Package for the Tank Waste Remediation System Environmental Impact Statement, WHC-SD-WM-EV-102, Rev. 0, Westinghouse Hanford Company, Richland, Washington.

WHC, 1995c, Fiscal Year 1996 Tank Waste Remediation System Tank Waste Analysis Plan, WHC-SD-WM-PLN-101, Rev. 0, Westinghouse Hanford Company, Richland, Washington. 


\subsection{CODE OF FEDERAL REGULATIONS AND FEDERAL REGISTER}

40 CFR 265, "Interim Status Standards for Owners and Operators of Hazardous Waste Treatment, Storage, and Disposal Facilities," Title 40, Code of Federal Regulations, Part 265, as amended, U.S. Environmental Protection Agency, Washington, D.C.

40 CFR 268, "Land Disposal Restrictions," Title 40, Code of Federal Regulations, Part 268, as amended, U.S. Environmental Protection Agency, Washington, D.C.

40 CFR 761, "Polychlorinated Biphenyls (PCB) Manufacturing, Processing, Distribution in Commerce, and Use Prohibitions," Title 40, Code of Federal Regulations, Part 761, as amended, U.S. Environmental Protection Agency, Washington, D.C.

55 FR 22520, 1990, "Land Disposal Restrictions for Third Scheduled Wastes; Final Rule," Federal Register, Vol. 55, pp. 22627, (June 1, 1990).

57 FR 37194, "Land Disposal Restrictions for Newly Listed Wastes and Hazardous Debris," Federal Register, Vol. 57, pp. 37194.

58 FR 29860, 1993, "Land Disposal Restriction for Ignitable and Corrosive Characteristic Wastes Whose Treatment Standards Were Vacated," Federal Register, Vol. 58, pp. 29860, (May 24, 1993).

59 FR 47982, 1994, "Universal Treatment Standards," Federal Register, Vol. 59, pp. 47982.

60 FR 242, 1994, "Technical Correction to Universal Treatment Standards," Federal Register, Vol. 60, pp. 242.

60 FR 31115, 1995, "Hazardous Waste Management System; Identification and Listing of Hazardous Waste; Final Exclusion," Federal Register, Vol. 60, pp. 31115.

61 FR 15660, 1996, "Land Disposal Restrictions Phase III--Decharacterized Wastewaters, Carbonate Waste, and Spent Potliners," Federal Register, Vol. 61, pp. 15660.

61 FR 15666, 1996, "Land Disposal Restrictions Phase III--Decharacterized Wastewaters, Carbonate Waste, and Spent Potliners," Federal Register, Vol. 61, pp. 15666.

61 FR 18588, "Extension of the Policy on Enforcement of RCRA Sec. 3004(j) Storage Prohibition at Facilities Generating Mixed Radioactive/Hazardous Waste," Federal Register, Vol. 61, pp. 18588.

61 FR 33680, 1996, "Land Disposal Restrictions Phase III--Decharacterized Wastewaters, Carbonate Waste, and Spent Potliners," Federal Register, Vol. 61, pp. 33680. 
61 FR 35419, 1996, "Land Disposal Restrictions Phase III--Decharacterized Wastewaters, Carbonate Waste, and Spent Potliners," Federal Register, Vol. 61, pp. 35419.

61 FR 43924, 1996, "Emergency Revision of the Land Disposal Restrictions (LDR) Phase III Treatment Standards for Listed Hazardous Waste from Carbonate Production; Final Rule," Federal Register, Vol. 61, pp. 43924.

61 FR 60704, 1996, "Termination of Review of Every Petition to EPA for a No-Migration Determination for the Waste Isolation Pilot Plant (WIPP) Under the Resource Conservation and Recovery Act," Federal Register, Vol. 61, pp. 60704.

62 FR 7502, 1997, "Land Disposal Restrictions: Corrections of Tables; Treatment Standards for Hazardous Wastes and Universal Treatment Standards," Federal Register, Vol. 62, pp. 7502.

\subsection{FEDERAL AND STATE ACTS}

Atomic Energy Act of 1954, as amended, 42 USC 2011.

Comprehensive Environmental Response, Compensation, and Liability Act of 1980, as amended, 42 USC 9601 et seq.

Federal Facility Compliance Act of 1992, as amended, 42 USC 6901, et seq.

National Environmental Policy Act of 1969, as amended, 42 USC 4321, et seq.

Resource Conservation and Recovery Act of 1976, as amended, 42 USC 6901, et seq.

State of Washington Hazardous Waste Management Act of 1976, as amended, Revised Code of Washington, Chapter 70.105, Olympia, Washington.

Toxic Substances Control Act of 1976, 15 USC 2601, et seq.

\subsection{WASHINGTON STATE REGULATIONS}

Ecology, Technical Information Memorandum 86-3, Treatment by Generators (revised July, 1993), Hazardous Waste and Toxics Reduction Program, Technical Assistance and Policy Sections, Washington State Department of Ecology, Olympia, Washington.

WAC-173-303, "Dangerous Waste Regulations," Washington Administrative Code, as amended. (WAC 173-303-140 covers land disposal restrictions.) 
DOE/RL-97-14

This page intentionally left blank. 
DOE/RL-97-14

APPENDIX A

DATA IN NATIONAL DATABASES 


\section{DOE/RL-97-14}

This page intentionally left blank.

A-ii 


\section{APPENDIX A}

\section{DATA IN NÁTIONAL DATABASE}

Under the Federal Facility Compliance Act, Hanford is exempt from preparing a site treatment plan. This is because the Hanford Federal Facility Agreement and Consent Order I (Tri-Party Agreement) (Ecology et al. 1996) and specifically the Site Land Disposal Restriction Report fulfill this requirement. Nevertheless, the Hanford Site does support the submission of waste data and information to databases that support both the Mixed Waste Inventory Report and the site treatment plans. The scope and objective of each national database are as follows:

- The Mixed Waste Inventory Report database contains inventories and detailed characteristics of each waste stream. The objective of the report is to determine the required land disposal restrictions treatment technologies for each waste stream.

- The site treatment plan database captures the inventories and required treatment technologiès from the Mixed Waste Inventory Report database. The objective of this database is to establish the national treatment configuration for low-level mixed waste.

A one-to-one correspondence exists between the Mixed Waste Inventory Report and the site treatment plan database for the Hanford Site waste streams.

This appendix summarizes the data that are presented in the October 1996 update to the Mixed Waste Inventory Site Treatment Plan database. This information is presented in two sections: Assumptions and data summary. The assumptions specify the technologies required to meet land disposal restrictions. These assumptions are based on a detailed analysis of each waste stream in the Mixed Waste Inventory Report. The data summary provides inventories and projections of mixed waste, organized by treatment technologies.

\subsection{ASSUMPTIONS FOR THE UPDATE TO THE NATIONAL SITE TREATMENT PLAN DATABASE}

The treatment and processing assumptions for Hanford's update to the Site Treatment Plan database are presented below. As already stated, the U.S. Department of Energy, Headquarters will use the proposed site treatment plan database as a tool to establish the national treatment configuration. as follows:

The assumptions are organized by waste management program or waste-generating facility,
A. Mixed high-level and tank waste
B. Transuranic (TRU) mixed waste
C. Mixed low-level waste (LLW) 

D. Liquid effluents
E. Wastes managed by the generator.

The assumptions for each program or facility are presented separately.

\section{A. OPTIONS FOR MIXED HLW AND TANK WASTE STREAMS}

The technical strategy for treatment and disposal of tank waste is based on the January 1994 amendments to the Tri-Party Agreement. The details of this strategy are summarized in Paragraphs A.1 and A.2.

A.1. Future generation of tank waste at the Hanford Site is based on the following assumptions:

- The fuel reprocessing plant [Plutonium-Uranium Extraction (PUREX) Plant] is not restarted

- The irradiated fuel remains in wet storage

- Pumpable liquids are transferred from single- to double-shell tanks

- Newly generated waste will be stored in double-shell tanks (DSTs)

- Volume reduction of stored DST waste will continue.

A.2. The current technical baseline for disposal of tank waste is to retrieve and process all (more than $99 \%$ ) of the tank waste using a two-phase approach. Most of the required processing capability will be designed, constructed, operated, and financed by private contractors. The demonstration phase (Phase I) facilities for supernatant (liquid) pretreatment and low-activity waste (LAW) and high-level waste (HLW) immobilization are scheduled to begin operation in June 2002 and will process waste through 2011. HLW sludge will be pretreated in-tank using water washing and caustic leaching as appropriate. Up to $13 \%$ of the supernatant and $6 \%$ of the sludge will be processed during Phase I. Full-scale production facilities, including out-of-tank sludge pretreatment, are scheduled to begin operating in 2012. These facilities will be sized to complete immobilization of LAW by 2024 and HLW by 2028 to meet current Tri-Party Agreement milestones.

The pretreatment processes separate the majority of the radioactivity contained in the tank waste volume for LAW immobilization. The current technical baseline uses settling/decanting to separate solids from the liquids, ion exchange to reduce the radioactivity in the supernatants, and caustic leaching to reduce the volume of HLW sludge requiring vitrification. Other pretreatment processes that have been evaluated in the Tank Waste Remediation System Environmental Impact Statement included acid dissolution, advanced separations, and organic destruction. 


\section{B. OPTIONS FOR MIXED TRU WASTE STREAMS}

Mixed TRU waste that meets the Waste Isolation Pilot Plant's (WIPP) Waste Acceptance Criteria can be shipped directly to WIPP without LDR treatment. This is because legislation exists that exempts the WIPP from LDR treatment requirements (61 FR 60704).

B.1 Waste that does not contain ignitable characteristics or Toxic Substances Control Act(TSCA-) regulated polychlorinated biphenals (PCBs) will be repackaged and processed at the Waste Receiving and Processing Facility Module 1 (Option RL-07) to meet the WIPP waste acceptance criteria.

B.2 Waste that contains ignitable characteristics (D001) or TSCA-regulated PCBs (i.e., TRUcontaminated hydraulic fluids) will be treated to remove the ignitable characteristic and destroy the PCBs. The treatment is required to meet the WIPP Waste Acceptance Criteria. A facility for providing this treatment capability has yet to be determined.

\section{OPTIONS FOR CONTAINERIZED MIXED LOW-LEVEL WASTE STREAMS}

\section{C.1 LDR Technology Specification Assumptions}

- Technology needs are driven by matrix and concentration of hazardous contaminants

- When needed, deactivation technology is achieved by thermal treatment or stabilization technologies required because of other contaminants. Need is determined by the Ignitable/Corrosive/Reactive-contaminant parameter.

- Where organics, or PCBs, and metals containing mercury are the contaminants present, thermal treatment technology would serve to both remove and/or destroy organics and remove mercury.

- Technology for metal-contaminated soil is stabilization rather than soil washing, etc.

- Debris matrices may be treated via destruction, immobilization, or removal technologies. Pretreatment via sorting/separation will likely be needed first.

- Homogeneous solids, soils, and labpacks that contain organics and are contaminated with mercury, lead, and/or alpha-bearing PCBs are difficult to treat thermally for the following reasons:

- Mercury, lead, and PCBs are volatile and heating them results in potential hazardous air emissions

- Alpha-bearing contaminants require special waste processing. 
The following waste streams may contain these difficult-to-treat waste types: RL-W021, RL-W124, RL-W054, RL-W051, RL-W132, RL-W144, RL-W044, and RL-W027.

\section{C.2 LDR Technology Specification}

The physical forms of mixed waste at the Hanford Site are as follows:

- Inorganic and organic process homogeneous solids, including particulates and sludges (homogeneous solids)

- Contaminated soils (soils)

- Labpacks

- Debris

- Elemental mercury

- Elemental lead

- Lead acid and cadmium batteries.

The treatment requirements for the first three physical forms are discussed together because the required technologies are similar. The treatment requirements for each of the other four physical forms are presented separately.

\section{C.2.1 Homogeneous Solids, Soils, and Labpacks.}

C.2.1.1. Labpacks containing organic solvents, oils, or PCBs will require, as a minimum, thermal treatment.

C.2.1.2. Organic contaminants are characterized by the following:

- Matrices laden with organics, i.e., absorbed oils and labpacked organics

- Solid or soil matrices contaminated with Resource Conservation and Recovery Act of 1976- (RCRA)-regulated organic contaminants

- TSCA-regulated levels of PCB contamination.

C.2.1.3. Homogeneous solids, soils, and labpacks contaminated with RCRA metals and organic I contaminants will be treated by thermal treatment and stabilization technologies. No volume 
I increase or decrease is expected as a result of treatment. The following waste streams make up this category.

$\begin{array}{llllll} & \text { RL-W021 } & \text { RL-W029 } & \text { RL-W031 } & \text { RL-W042 } & \text { RL-W043 } \\ & \text { RL-W044 } & \text { RL-W045 } & \text { RL-W051 } & \text { RL-W053 } & \text { RL-W054 } \\ & \text { RL-W061 } & \text { RL-W063 } & \text { RL-W064 } & \text { RL-W114 } & \text { RL-W119 } \\ & \text { RL-W122 } & \text { RL-W124 } & \text { RL-W125 } & \text { RL-W132 } & \text { RL-W144 } \\ & \text { RL-W149 } & \text { RL-W153 } & \text { RL-W178 } & \text { RL-W181 } & \text { RL-W191 } \\ & \text { RL-W193 } & \text { RL-W196 } & \text { RL-W201 } & \text { RL-W212 } & \text { RL-W215 } \\ \text { | } & \text { RL-W218 } & \text { RL-W219 } & \text { RL-W220 } & \text { RL-W584 } & \text { RL-W590 } \\ \text { | } & \text { RL-W623 } & \text { RL-W626 } & \text { RL-W638 } & \text { RL-W645 } & \text { RL-W646 } \\ \text { I } & \text { RL-W657 } & \text { RL-W698 } & \text { RL-W701 } & \text { RL-W702 } & \text { RL-W703 } \\ \text { | } & \text { RL-W731 } & \text { RL-W732 } & \text { RL-W751 } & & \end{array}$

C.2.1.4. Homogeneous solids, soils, and labpacks containing organic contaminants but not RCRA metals will be treated by a thermal treatment technology. Stabilization of the residues

I from thermal treatment is not planned. No volume increase or decrease is expected as a result of treatment. The following waste streams make up this category.

$\begin{array}{lllll}\text { RL-W017 } & \text { RL-W027 } & \text { RL-W039 } & \text { RL-W040 } & \text { RL-W046 } \\ \text { RL-W049 } & \text { RL-W050 } & \text { RL-W052 } & \text { RL-W087 } & \text { RL-W065 } \\ \text { RL-W066 } & \text { RL-W067 } & \text { RL-W092 } & \text { RL-W096 } & \text { RL-W099 } \\ \text { RL-W090 } & \text { RL-W100 } & \text { RL-W108 } & \text { RL-W110 } & \text { RL-W123 } \\ \text { RL-W130 } & \text { RL-W140 } & \text { RL-W143 } & \text { RL-W145 } & \text { RL-W148 } \\ \text { RL-W154 } & \text { RL-W170 } & \text { RL-W174 } & \text { RL-W177 } & \text { RL-W180 } \\ \text { RL-W182 } & \text { RL-W194 } & \text { RL-W197 } & \text { RL-W202 } & \text { RL-W206 } \\ \text { RL-W213 } & \text { RL-W216 } & \text { RL-W221 } & \text { RL-W223 } & \text { RL-W230 } \\ \text { RL-W231 } & \text { RL-W580 } & \text { RL-W583 } & \text { RL-W585 } & \text { RL-W586 } \\ \text { RL-W587 } & \text { RL-W588 } & \text { RL-W591 } & \text { RL-W595 } & \text { RL-W610 } \\ \text { RL-W613 } & \text { RL-W624 } & \text { RL-W627 } & \text { RL-W628 } & \text { RL-W632 } \\ \text { RL-W634 } & \text { RL-W641 } & \text { RL-W647 } & \text { RL-W653 } & \text { RL-W654 } \\ \text { RL-W661 } & \text { RL-W682 } & \text { RL-W696 } & \text { RL-W704 } & \text { RL-W705 } \\ \text { RL-W733 } & \text { RL-W734 } & \text { RL-W735 } & \text { RL-W752 } & \end{array}$


C.2.1.5. Homogeneous solids, soils, and labpacks contaminated with RCRA metals, ignitable/corrosive/reactive materials, and/or contaminants that fall under Washington State codes, will be treated by applying stabilization technology. A volume increase of 2:1 is expected I as a result of treatment. The following waste streams make up this category (an asterisk indicates I a remote-handled waste stream).

$\begin{array}{llllll} & \text { RL-W018 } & \text { RL-W019 } & \text { RL-W022 } & \text { RL-W047 } & \text { RL-W048 } \\ & \text { RL-W094 } & \text { RL-W098 } & \text { RL-W089 } & \text { RL-W121 } & \text { RL-W171 } \\ & \text { RL-W173 } & \text { RL-W198 } & \text { RL-W203 } & \text { RL-W205 } & \text { RL-W214 } \\ \text { I } & \text { RL-W222 } & \text { RL-W224 } & \text { RL-W225 } & \text { RL-W229 } & \text { RL-W581 } \\ \text { I } & \text { RL-W589 } & \text { RL-W592 } & \text { RL-W612 } & \text { RL-W614 } & \text { RL-W616 } \\ \text { I } & \text { RL-W629 } & \text { RL-W631 } & \text { RL-W633 } & \text { RL-W635 } & \text { RL-W637 } \\ \text { I } & \text { RL-W640 } & \text { RL-W642 } & \text { RL-W643 } & \text { RL-W648 } & \text { RL-W649 } \\ \text { I } & \text { RL-W650 } & \text { RL-W652 } & \text { RL-W655 } & \text { RL-W656 } & \text { RL-W660 } \\ \text { | } & \text { RL-W662 } & \text { RL-W697 } & \text { RL-W700 } & \text { RL-W706 } & \text { RL-W708 } \\ \text { | } & \text { RL-W709 } & \text { RL-W710 } & \text { RL-W716* } & \text { RL-W728 } & \text { RL-W730 } \\ \text { | } & \text { RL-W736 } & \text { RL-W737 } & \text { RL-W738 } & \text { RL-W739 } & \text { RL-W740 } \\ \text { | } & \text { RL-W753 } & \text { RL-W753 } & & & \end{array}$

C.2.1.6. Inorganic homogeneous solids and soils contaminated with mercury will be treated by stabilization technology. Additional characterization of the waste will be performed to verify that the mercury concentration in the waste matrix is less than the regulated level of $260 \mathrm{mg} / \mathrm{L}$. Waste that is determined to exceed $260 \mathrm{mg} / \mathrm{L}$ will be identified as separate waste streams. A volume increase of $2: 1$ is expected as a result of treatment. The waste will be treated by retorting and/or roasting technologies. The selection of a facility with retorting and roasting capability will be conducted when these waste streams are identified and accumulated. The following waste streams make up this category.

$\begin{array}{llllll} & \text { RL-W020 } & \text { RL-W038 } & \text { RL-W120 } & \text { RL-W172 } & \text { RL-W183 } \\ \text { | } & \text { RL-W195 } & \text { RL-W204 } & \text { RL-W207 } & \text { RL-W593 } & \text { RL-W594 } \\ \text { | } & \text { RL-W596 } & \text { RL-W615 } & \text { RL-W617 } & \text { RL-W618 } & \text { RL-W619 } \\ \text { | } & \text { RL-W620 } & \text { RL-W622 } & \text { RL-W630 } & \text { RL-W658 } & \text { RL-W707 } \\ \text { | } & \text { RL-W729 } & & & & \end{array}$


C.2.1.7. Stabilization will be used to process containerized, dewatered fuel-fabrication sludge from the 183-H dewatering basins. This is a listed waste containing low concentrations of formic acid. A petition to waive the formic acid incineration LDR treatment standard using a 1 demonstration of equivalent treatment is being prepared for this waste. A volume increase of 2:1 I is expected as a result of treatment. The following waste streams make up this category.

\section{RL-W068 RL-W069 RL-W070}

C.2.1.8. Tank farm backlog soils are assumed to meet the best demonstrated available technology (BDAT) concentration levels for organic solvents. Sampling and analysis of the soil is planned to I support a petition to the Washington State Department of Ecology (Ecology) for concurrence I that the waste stream meets the BDAT concentration levels. No volume increase or decrease is I expected as a result of treatment. The waste streams representing this category are the following:

|

$\begin{array}{lll}\text { I } & \text { RL-W184 } & \text { RL-W185 }\end{array}$

1

C.2.2 Debris. Debris waste, including debris contaminated with low concentrations of organic I solvents, oils, or PCBs, and backlog debris will be treated by immobilization technologies. The planned technology is shred-grout immobilization. Debris waste that contains no or low concentrations of these hazardous constituents will be pretreated by screening to remove particulates.

An alternative treatment strategy under evaluation is macroencapsulation using sealed I polyethylene containers. Compaction technology will also be applied. Ecology has concurred I with placing waste in sealed polyethylene containers that meet the BDAT standard for I macroencapsulation. The expected volume increase is $2: 1$.

The following waste streams make up this category (an asterisk indicates a remotehandled waste stream).

$\begin{array}{lllll}\text { RL-W023 } & \text { RL-W024 } & \text { RL-W025 } & \text { RL-W026 } & \text { RL-W028 } \\ \text { RL-W030 } & \text { RL-W032 } & \text { RL-W041 } & \text { RL-W033 } & \text { RL-W036 } \\ \text { RL-W055 } & \text { RL-W056 } & \text { RL-W057 } & \text { RL-W058 } & \text { RL-W059 } \\ \text { RL-W060 } & \text { RL-W093 } & \text { RL-W095 } & \text { RL-W097 } & \text { RL-W113 } \\ \text { RL-W115 } & \text { RL-W116 } & \text { RL-W117 } & \text { RL-W118 } & \text { RL-W126 } \\ \text { RL-W127 } & \text { RL-W128 } & \text { RL-W129 } & \text { RL-W131 } & \text { RL-W141 } \\ \text { RL-W142 } & \text { RL-W146 } & \text { RL-W150 } & \text { RL-W151 } & \text { RL-W175 } \\ \text { RL-W176 } & \text { RL-W186 } & \text { RL-W187 } & \text { RL-W188 } & \text { RL-W189 }\end{array}$


DOE/RL-97-14

\begin{tabular}{|c|c|c|c|c|c|}
\hline & RL-W199 & RL-W200 & RL-W208 & RL-W209 & RL-W210 \\
\hline & RL-W211 & RL-W226 & RL-W227 & RL-W228 & RL-W232 \\
\hline I & RL-W233 & RL-W582 & RL-W597 & RL-W598 & RL-W599 \\
\hline 1 & RL-W600 & RL-W60I & RL-W602 & RL-W603 & RL-W604 \\
\hline 1 & RL-W605 & RL-W606 & RL-W607 & RL-W608 & RL-W609 \\
\hline 1 & RL-W621 & RL-W636 & RL-W666 & RL-W664 & RL-W665 \\
\hline 1 & RL-W666 & RL-W667 & RL-W668 & RL-W669 & RL-W670 \\
\hline I & RL-W671 & RL-W672 & RL-W673 & RL-W674 & RL-W675 \\
\hline 1 & RL-W676 & RL-W677 & RL-W678 & RL-W679 & RL-W680 \\
\hline | & RL-W68I & RL-W683 & RL-W684 & RL-W685 & RL-W686 \\
\hline $\mid$ & RL-W687 & RL-W688 & RL-W689 & RL-W690 & RL-W691 \\
\hline & RL-W692 & RL-W693 & RL-W694 & RL-W695 & RL-W717* \\
\hline & RL-W718* & RL-W719* & RL-W720* & RL-W721* & RL-W722 \\
\hline & RL-W723 & RL-W724 & RL-W725 & RL-W726 & RL-W727 \\
\hline & RL-W742* & RL-W743* & RL-W744* & RL-W754 & \\
\hline
\end{tabular}

C.2.3 Elemental Mercury. Waste containing elemental mercury will be treated by amalgamation followed by encapsulation. A volume increase of $15: 1$ is expected as a result of I applying these technologies. The following waste stream makes up this category.

RL-W037

C.2.4 Elemental Lead. Elemental lead will be macroencapsulated. Decontamination and recycling technologies are not planned. The following waste streams make up this category.

$\begin{array}{llllll}\text { | } & \text { RL-W035 } & \text { RL-W062 } & \text { RL-W152 } & \text { RL-W179 } & \text { RL-W192 } \\ \text { | } & \text { RL-W217 } & \text { RL-W235 } & \text { RL-W61 1 } & \text { RL-W625 } & \text { RL-W639 } \\ \text { | } & \text { RL-W644 } & \text { RL-W711 } & \text { RL-W712 } & \text { RL-W713 } & \text { RL-W741 } \\ \text { | } & \text { RL-W755 } & & & & \end{array}$


C.2.5 Batteries. Lead acid and cadmium batteries will be encapsulated in a shred-grout form. Neutralization may be required. A volume increase of $2: 1$ is expected as a result of treatment. I The following waste streams make up this category.

$$
\text { I RL-W034 RL-W091 } \quad \text { RL-W147 } \quad \text { RL-W714 }
$$

1 C.2.6 Reactive, explosive, and propellant waste will be deactivated by the generator before storage. Additional stabilization treatment is anticipated to meet LDRs.

\section{C.3 Preferred Treatment Option}

I C.3.1 Contact-handled waste streams that require thermal treatment will be processed by a commercial facility (RL-04). The second option is to ship this waste to the planned Idaho Waste I Processing Facility (IN-015) at the Idaho National Engineering Laboratory. The third option is to ship the waste to the planned Oak Ridge treatment facility at Oak Ridge, Tennessee. Under any option, additional treatment will be provided at the facility as required to ensure that the returned waste residues are in an acceptable form for disposal.

I C.3.2 The remaining contact-handled waste streams (those that do not require thermal treatment) will be processed at a commercial facility providing non-thermal mixed waste treatment capability (RL-03), WRAP 1, or T Plant. These services are planned to be available by September 1999.

The present plan is that debris and dewatered fuel fabrication sludge will be processed at a commercial facility. Small-volume waste streams, such as elemental mercury and batteries, will be treated at WRAP 1. A second option is to treat the small-volume waste streams at a single DOE complex-wide facility designed to handle these waste streams. Elemental lead will be treated at T Plant.

C.3.3 Technology development (RL-10) is needed to determine the appropriate treatment technologies for remote-handled waste streams, including long-length equipment from the tanks. One strategy is to initially process this waste at a remote-handled facility with sorting, decontamination, and packaging capabilities. Waste containers would be opened and the contents examined. Contaminated equipment, metal components, and debris would be treated by decontamination to contact-handled levels. Contact-handled waste items would be sorted into streams and packaged for LDR treatment at a facility that accepts contact-handled waste. Any remaining remote-handled waste items would be macroencapsulated. Petitions to waive the thermal treatment requirements for remote-handled organic contaminated residues and soils may be necessary. 


\section{OPTION FOR PROCESS CONDENSATE}

The delisting petition filed with the U.S. Environmental Protection Agency for process condensate from the 242-A Evaporator was approved in June 1995. Therefore, after treatment, the process condensate is not a mixed waste and is not included in the national mixed waste inventory for strategic planning purposes.

\section{E. OPTIONS FOR WASTE STORED BY GENERATOR}

I Some mixed waste is stored by the waste generator. This is waste that, because of radiological, hazardous, or other unique conditions associated with the waste form, is presently I stored by the waste generators. To the extent possible, waste at the Pacific Northwest National I Laboratory (PNNL) will be packaged and shipped to the Central Waste Complex for long-term I storage. These waste streams are the following:

- Hexone

- Waste in the PUREX tunnels and canyon

- Waste at the Radiochemistry Engineering Laboratory.

The treatment plan and technology strategy for each waste stream are presented in I Paragraphs E.1 through E.3.

\section{E.1 Hexone}

I The spent distillate bottoms have been incinerated at an offsite facility. Therefore, for practical purposes, the waste stream no longer exists.

Residues in vessels used in the previous distillation campaign will be packaged and sent to the Central Waste Complex for storage. Therefore, this residue is included in the projections for the mixed LLW streams. The waste is assumed to be debris because glass is present. Therefore, the planned treatment for the waste is stabilization through a commercial treatment service contract (Section C.3.2).

\section{| E.2 Waste in the PUREX Tunnels and Canyon}

| E.2.1. The specified LDR technology for PUREX tunnels silver waste and PUREX canyon/tunnels lead waste is encapsulation. The plan and schedule for implementation of a treatment facility has yet to be determined.

I E.2.2. The specified LDR technology for PUREX tunnels mercury waste is amalgamation. The plan and schedule for construction of the treatment facility have yet to be determined. Treatment 
of waste in the PUREX tunnel is beyond the scope of the PUREX deactivation project to be completed in September 1997. Waste from the tunnels will be handled along with the similar materials currently in the PUREX canyon when the PUREX Plant is dispositioned. The dispositioning of PUREX, along with treatment of the tunnel waste, is contingent on completion of the following:

- The sitewide land use plan

- The sitewide decontamination and decommissioning priority schedule

- The environmental impact statement, closure plans, and public participation.

A basis for the treatment plan of the waste associated with PUREX storage will be developed after these are implemented.

\section{| E.3 Radiochemistry Engineering Laboratory}

Nine mixed waste streams from this facility were identified in Calendar Year 1994. One waste stream is $\mathrm{HLW}$, four are remote-handled TRU waste, three are remote-handled LLW, and one is contact-handled LLW.

To the extent possible, these waste streams will be packaged and shipped to the Central Waste Complex for storage and eventual LDR treatment. The treatment plan and schedule for remaining mixed waste at the laboratory will be negotiated with Ecology.

\section{F. REFERENCES}

I 61 FR 60704, "Final Hanford Site Tank Waste Remediation System Environmental Impact Statement," Federal Register, Washington, D.C.

Ecology, EPA, and DOE, 1996, Hanford Federal Facility Agreement and Consent Order, Washington State Department of Ecology, U.S. Environmental Protection Agency, and U.S. Department of Energy, Olympia, Washington.

ORNL, 1996, Integrated Data Base Report-1995: U.S. Spent Nuclear Fuel and Radioactive Waste Imventories, Projections, and Characteristics, Oak Ridge National Laboratory, Lockheed Martin Energy Research Corp., Oak Ridge, Tennessee.

Resource Conservation and Recovery Act of 1976, 42 U.S.C. 6901, et seq. 


\subsection{HANFORD FACILITY WASTE STREAM SUMMARY}

This section summarizes the HLW, mixed TRU waste, and mixed LAW, as presented in the October 1996 update to the national site treatment plan database. Because the waste types either are managed or will be processed differently, each is discussed separately.

This section uses the terms "inventory" and "projection." Inventory is the volume of waste in storage as of a specified date. The 5-year projection covers the waste generation for the following 5 years.

\section{HIGH-LEVEL WASTE}

At the Hanford Facility, waste in SSTs and DSTs consists of HLW, TRU waste, and mixed I LAW. However, the tanks are managed as if they contain HLW and are reported in the high-level category.

The inventory and 5-year projection for each double-shell tank waste stream are presented as a material balance in Table 1. The projections show that much of the dilute tank waste in I storage will be reduced in volume by evaporation. The data in this table are consistent with the | Integrated Data Base (ORNL 1996).

Table 1. Double-Shell Tank Waste 5-Year Material Balance $\left(\mathrm{m}^{3}\right)$.

\begin{tabular}{|l|c|c|c|c|}
\hline \multicolumn{1}{|c|}{ FFCA waste stream name } & Inventory & \multicolumn{2}{c|}{5 -year transactions } & Projection \\
& $12 / 31 / 94$ & Inputs & Outputs & $12 / 31 / 99$ \\
\hline PUREX aging waste & 7,116 & 0.00 & & 7,116 \\
\hline DST miscellaneous waste & 20,372 & 40,859 & & 61,201 \\
\hline DST double-shell slurry feed & 17,498 & 10,162 & & 27,660 \\
\hline DST dilute complexed & 3,543 & 11,254 & & 14,797 \\
\hline DST concentrated phosphate & 4,156 & 0.00 & & 4,156 \\
\hline DST PFP TRU solids & 269 & 35 & & 304 \\
\hline DST complex concentrate & 10,935 & 0.00 & & 10,935 \\
\hline DST double-shell slurry & 7,790 & 15.00 & & 7,805 \\
\hline DST PUREX CRW solids & 2,498 & 49.00 & & 2,547 \\
\hline Output to Evaporator & & & 57,662 & $\mathbf{- 5 7 , 6 6 2}$ \\
\hline Total & 74,177 & 62,374 & 57,662 & $78,889^{\mathbf{2}}$ \\
\hline
\end{tabular}

"Value is consistent with the Integrated Data Base, Rev. 12

$\mathrm{CRW}=$ (fuel) cladding removal waste, DST $=$ double-shell tank,

FFCAct $=$ Federal Facility Compliance Act, PFP = Plutonium Finishing Plant

TRU $=$ transuranic 


\section{MIXED TRU WASTE}

A volume of designated mixed TRU waste at the Hanford Site is slightly more than 1 percent of the stored TRU waste volume. Designation for mixed waste has occurred only recently and is related to the DOE Byproduct Material Rulemaking of 1987. About one-fourth of the TRU waste generated between January 1, 1987, and December 31, 1995, has been designated as mixed waste.

The total inventory of mixed TRU waste in the proposed site treatment plan database is presented in Table 2. Of this inventory, $77 \mathrm{~m}^{3}$ contain ignitable (D001) characteristics and/or PCB contaminants. To meet the waste acceptance criteria for disposal at the WIPP, the waste will need to be treated to remove the ignitable characteristic and destroy the PCBs. A preferred treatment option has not been determined.

Table 2. Hanford Site Mixed TRU Waste Inventory.

\begin{tabular}{l|lr|}
\cline { 2 - 3 } & Inventory as of December 31, 1994 & $288.2 \mathrm{~m}^{3}$ \\
1 & Revisions/Corrections (+/-) & $0 \mathrm{~m}^{3}$ \\
1 & $7.7 \mathrm{~m}^{3}$ \\
\cline { 2 - 3 } & Annual additions in Calendar Year 1995 & $295.9 \mathrm{~m}^{3}$ \\
\cline { 2 - 3 } &
\end{tabular}

\section{MIXED LOW-LEVEL WASTE}

Mixed LLW at the Hanford Facility encompasses the following:

- Mixed waste in storage under the cognizance of RUST Federal Services of Hanford, Inc. (hereafter referred to as "RUST")

- Small amounts of mixed waste that are stored by generators.

I Waste storage by generator accounts for less than $10 \mathrm{~m}^{3}$ of the mixed LLW inventory. Of the I waste under the cognizance of RUST, only one waste stream is a "direct process"1 waste stream. This waste is solidified, dewatered fuel fabrication sludge and is commonly known as " $183-\mathrm{H}$

I basin waste." The other waste streams under RUST are "less proximate to the physical processes of producing or utilizing special nuclear material,"' therefore they are organized by treatability I groups in the national site treatment plan database. The inventory of mixed LLW is presented in Table 3.

\footnotetext{
${ }^{1}$ Supplemental information to the Byproduct Material Rulemaking, May 1, 1987. The updated reference mixed $L L W$ inventory is presented in Table 5 . The inventory excludes contributions of those waste streams regulated under the Toxic Substance Control Act.
} 
Table 3. Referenced Hanford Site Mixed Low-Level Waste Inventory. $\left(\mathrm{m}^{3}\right)$

Referenced inventory as of December 31, 1994

$6,329.55$

Revisions/corrections (+/-)

$1,074.00$

Annual additions in Calendar year 1995

612.9

Additions from $1 / 1 / 96$ to $6 / 30 / 96$

130.32

Additions from 7/1/96 to $9 / 30 / 96$ treated to an immobilized form

22.77

Referenced inventory as of June 30,1996

$8,169.54$

The revision/correction in Table 3 is the result of the following:

- A volume of $1.065 \mathrm{~m}^{3}$ reflects overpacking of $183-\mathrm{H}$ basin waste into $85-\mathrm{gal}$ containers

- A volume of $20 \mathrm{~m}^{3}$ was added for waste that still has to be characterized by physical and contaminant characteristics

- A volume of $9 \mathrm{~m}^{3}$ was subtracted because one waste stream was eliminated through waste transfer from the Fast Flux Test Facility to RUST.

Low-level mixed waste (LLMW) generation fell in calendar year 1995 because the tank farm backlog generation was completed in 1994. Nevertheless, LLMW generation in calendar year 1995 was slightly higher than non-backlog waste generation in calendar year 1994. Table 4 presents a breakdown of LLMW by source.

Table 4. Sources of LLMW in 1995.

\begin{tabular}{|c|c|}
\hline Source & Annual volume $\left(\mathrm{m}^{3}\right)$ \\
\hline Offsite laboratories & 76.58 \\
\hline Maintenance and operations contractors & 472.90 \\
\hline $\begin{array}{l}\text { Environmental Restoration and } \\
\text { decontamination/decommissioning }\end{array}$ & 26.71 \\
\hline $\begin{array}{l}\text { Research and Development } \\
\text { Pacific Northwest National Laboratory }\end{array}$ & 36.71 \\
\hline Total & 612.90 \\
\hline
\end{tabular}


The distribution of the LLMW inventory and 5 -year projection by physical form is presented in Table 5. As expected, the majority of nonsludge waste is debris. The 5-year projection period for LLMW is from 7/1/96 to $9 / 30 / 2001$.

From a national perspective, the Hanford Site has the fifth largest inventory of LLMW, moving up two places from last year. Even without overpacking of existing waste, the Hanford Site's LLMW inventory is increasing at a higher rate than any other site.

Table 5. Physical Distribution of LLMW.

\begin{tabular}{|c|c|c|c|}
\hline Physical Form & Inventory & $\begin{array}{l}\text { Five-Year } \\
\text { Projection }\end{array}$ & Total \\
\hline Batteries & 1.86 & 2.27 & 4.13 \\
\hline Elementąl hazardous metals & 240.03 & 134.50 & 374.53 \\
\hline Elemental mercury & 1.45 & 0.66 & 2.11 \\
\hline Explosives/propellants & 0.00 & 1.14 & 1.14 \\
\hline Heterogeneous debris & 808.78 & $1,678.04$ & $2,486.82$ \\
\hline Immobilized forms & 86.47 & 0.00 & 86.47 \\
\hline Inorganic debris & 535.31 & $1,145.29$ & $1,680.60$ \\
\hline Inorganic homogeneous solids & $3,779.41$ & 260.65 & $4,040.06$ \\
\hline Lab packs & 291.35 & 123.67 & 415.02 \\
\hline Organic debris & $1,690.50$ & 585.92 & $2,276.42$ \\
\hline Organic liquids & 1.04 & 0.00 & 1.04 \\
\hline Reactive metals & 5.43 & 0.00 & 5.43 \\
\hline Soil/gravel & 476.38 & 244.01 & 720.39 \\
\hline Unknown/other debris & 145.45 & 0.00 & 145.45 \\
\hline Unknown/other homogeneous solids & 25.32 & 24.30 & 49.61 \\
\hline Unknown/other matrix & 60.15 & 0.00 & 60.15 \\
\hline Unknown/other solids & 20.62 & 0.00 & 20.62 \\
\hline Hanford total & $8,169.54$ & $4,200.44$ & $12,369.98$ \\
\hline
\end{tabular}

1 Reference: Integrated Data Base, Rev. 12. 

The list of mixed low-level waste streams in the national Site Treatment Plan database is presented in Table 6 . The waste streams are sorted by the following categories.

- Waste streams targeted to a non-thermal treatment facility (see section C.3.2)

- Waste streams targeted to thermal treatment facility (see section C.3.1)

- Waste streams needing technology development (see section C.3.3)

- Waste streams that have been treated by macroencapsulation.

Subtotals are provided for each category.

The key to each physical matrix code is presented in Table 7. 
Tabie 6. Mixed low-level waste streams by targeted treatment capability (m3) (sheet 1 of 10 ).

\begin{tabular}{|c|c|c|c|c|c|}
\hline WS_id & Waste Stream Name & $\begin{array}{l}\text { Matrix } \\
\text { Code }\end{array}$ & $\begin{array}{l}\text { Inven- } \\
\text { tory }\end{array}$ & $\begin{array}{l}5 \text { yr pro- } \\
\text { jection }\end{array}$ & $\begin{array}{l}\text { Remaining } \\
\text { life-cycle } \\
\text { projection }\end{array}$ \\
\hline RL-W001 & HEXONE & $S 3223$ & 0.00 & 0.00 & 0.00 \\
\hline RL-W003 & ALKALI METAL WASTE (LSA) & $\times 7510$ & 0.00 & 0,00 & 0.00 \\
\hline RL-W018 & TC METAL SLUDGES/DRY PARTICULATE & 53190 & 105.85 & 40.42 & 269.94 \\
\hline RL-W019 & TC METAL SLUDGES/DRY PARTICULATE (AS) & $\$ 3119$ & 0.89 & 0.34 & 2.27 \\
\hline RL-WO20 & TC METAL SLUDGES/DRY PARTICULATE (HG) & $\$ 3129$ & 47.39 & 18,10 & 120.85 \\
\hline RL-WO22 & NON-TC METAL/SOLVENT SLUDGES/DRY PART. & $\$ 3113$ & 15.22 & 5.01 & 38.82 \\
\hline $\mathrm{RL}$ - $\mathrm{WO} 23$ & TC METAL INORGANIC SOLID DEBRIS & $\$ 5490$ & 19.63 & 30.36 & 72.11 \\
\hline $\mathrm{RL}-\mathrm{W0} 24$ & TC METAL INORGANIC SOLID DEBRIS (CA) & $\$ 5420$ & 3.36 & 10.29 & 54.17 \\
\hline $\mathrm{RL}-\mathrm{WO} 25$ & TC METAL INORGANIC SOLID DEBRIS (HG) & $\$ 5420$ & 2.63 & 8.05 & 42.34 \\
\hline $\mathrm{RL}-\mathrm{W0} 26$ & TC METAL INORG. GLASS SOLID DEBRIS (HG) & $\$ 5190$ & 4.73 & 14.48 & 76.23 \\
\hline $\mathrm{RL}-\mathrm{W0} 2 \mathrm{~B}$ & NON-TC METAL/SOLVENT INORG. SOLID DEBRIS & $\$ 5420$ & 3.07 & 9.40 & 49.46 \\
\hline RL-WO 30 & TC METAL ORGANIC SOLID DEERIS (CA) & $\$ 5490$ & 12.72 & 19.67 & 46.73 \\
\hline $\mathrm{RL}-$ WO 32 & TC METAL ORGANIC SOLID DEBRIS (HG) & 55440 & 14.28 & 18.95 & 118.63 \\
\hline $\mathrm{RL}-$ - 033 & NON-TC MET/SOLV. ORG. SOLID DEBRIS (CA, LO & $\$ 5490$ & 1.05 & 1.62 & 3.84 \\
\hline $\mathrm{RL}-$ WO 34 & LEAD ACID BATTERIES & $\times 7410$ & 1.24 & 0.77 & 0.07 \\
\hline RL-wo 35 & RADIOACTIVE LEAD SOLIDS & $\times 7219$ & 165.74 & 97.73 & 131.14 \\
\hline RL-W036 & RADIOACTIVE GLASS LEAD SOLIDS & 55122 & 0.63 & 1.93 & 10.16 \\
\hline RL-W0 37 & RADIOACTIVE ELEMENTAL MERCURY (CA) & $\times 7100$ & 1.45 & 0.15 & 0.09 \\
\hline $\mathrm{RL}-$ w0 38 & MERCURY SLUDGES/DRY PART. (HG>260MG/KG) & 53119 & 0.02 & 0.01 & 0.05 \\
\hline RL-W04 1 & NON-TC METAL/SOLVENT ORG. SOLID DEBRIS & S5440 & 13.19 & 17.50 & 109.55 \\
\hline RL-WO4 7 & TC METAL SOIL & 54200 & 1.95 & 1.28 & 1.93 \\
\hline RL-WO4E & NON-TC METAL/SOLVENT SOIL & $\$ 4200$ & 1.96 & 1.29 & 1.94 \\
\hline RL-w05 5 & SOLVENT INORGANIC SOLID DEERIS & $\$ 5490$ & 55.39 & 85.66 & 203.47 \\
\hline RL-W056 & SOLVENT INORGANIC SOLID DEBRIS (CA) & 55410 & 58.31 & 90.19 & 214.22 \\
\hline RL-W057 & SOLVENT ORGANIC SOLID DEBRIS & 55390 & 227.31 & 301.59 & $1,888.44$ \\
\hline RL-W05日 & SOLVENT/TC METAL ORGANIC SOLID DEBRIS & 55440 & 13.04 & 17.30 & 108.30 \\
\hline RL-W059 & SOLVENT/TC METAL ORG. SOLID DEBRIS (CA) & $\$ 5490$ & 6.48 & 10.02 & 23.80 \\
\hline RL-W0 60 & SOLVENT ORGANIC SOLID DEBRIS (CA) & $\$ 5440$ & 34.01 & 45.12 & 282.54 \\
\hline RL-W062 & SOLVENT RADIOACTIVE LEAD SOLIDS & $\times 7219$ & 2.66 & 1.57 & 2.10 \\
\hline $\mathrm{RL}-$ W068 & 183-H SOLAR BASIN WASTE, TYPE 1 & $\$ 3122$ & 832.65 & 0.00 & 0.00 \\
\hline RL-W069 & 183-H SOLAR BASIN WASTE, TYPE 2 & 53122 & 1.874 .13 & 0.00 & 0.00 \\
\hline RL-W070 & 183-H SOLAR BASIN WASTE, TYPE 3 & 53122 & 669.01 & 0.00 & 0.00 \\
\hline RL-W089 & TC METAL SLUDGES/DRY PARTICULATE (CA) & 53119 & 0.11 & 0.04 & 0.28 \\
\hline RL-W091 & CADMIUM BATTERIES & $\times 7420$ & 0.21 & 0.13 & 0.01 \\
\hline RL-W093 & WA REGULATED TC METAL SLUDGES/DRY PART. & 55490 & 18.89 & 29.21 & 69.39 \\
\hline RL-W094 & WA NON-TC METAL NON-SOLV. SLUDGES/DRY PA & 53190 & 36.76 & 14.04 & 93.74 \\
\hline RL-W095 & WA REGULATED IC METAL INORG. SOLID DEBRI & 55420 & 119.42 & 365.83 & $1,925.28$ \\
\hline RL-W097 & WA NON-TC METAL/SOLV. ORG. SOLID DEBRIS & 55440 & 10.58 & 14.04 & 87.93 \\
\hline RL-W098 & WA REGULATED TC METAL SOIL & 54100 & 9.12 & 6.01 & 9.06 \\
\hline RL-W113 & 183-H BASIN WASTE, TYPE 4 & 55490 & 15.00 & 23.20 & 55.10 \\
\hline RL-W115 & MLLW-ORGANIC DEBRIS-TC MET (AS) & 55440 & 1.24 & 1.65 & 10.30 \\
\hline RL-W116 & MLLW-ORGANIC DEBRIS-TC ORG & 35440 & $1: 82$ & 2.41 & 15.08 \\
\hline RL-W117 & MLLW-ORGANIC DEBRIS-PU LIST (L01)/TC MET & 35440 & 0.20 & 0.27 & 1.66 \\
\hline RL-W1 18 & MLLW-ORGANIC DEBRIS-TC ORG/TC MET (Hg) & 55440 & 1.05 & 1.39 & 8. 72 \\
\hline RL-W1 20 & MLLW-SOIL-TC MET (Hg) & 54200 & 0.20 & 0.13 & 0.20 \\
\hline
\end{tabular}


Table 6. Mixed low-level waste streams by targeted treatment capability (m3) (sheet 2 of 10 ).

\begin{tabular}{|c|c|c|c|c|c|}
\hline WS_id & Waste Stream Name & $\begin{array}{l}\text { Matrix } \\
\text { Code }\end{array}$ & $\begin{array}{l}\text { Inven- } \\
\text { tory }\end{array}$ & $\begin{array}{l}5 \text { yr pro- } \\
\text { Jection }\end{array}$ & $\begin{array}{l}\text { Remaining } \\
\text { life-cycle } \\
\text { projection }\end{array}$ \\
\hline$R L-W 121$ & MLLW-SOIL-TC MET (As) & 54100 & 0.21 & 0.14 & 0.21 \\
\hline RL-W126 & MLIW-ORGANIC DEBRIS-TC MET (Hq)/CA & 55440 & 0.20 & 0.27 & 1.66 \\
\hline RL-W127 & MLLW-ORGANIC DEBRIS-TC ORG/CA & 55440 & 1.04 & 1.38 & 8.65 \\
\hline RL-W1 28 & MLLW-ORGANIC DEBRIS-FU LIST (L01)/TC MET/CA & $\$ 5440$ & 0.21 & 0.28 & 1.73 \\
\hline RL-W1 29 & MLLW-ORGANIC DEBRIS-TC ORG/TC MET/CA & 55490 & 0.83 & 1.29 & 3.06 \\
\hline RL-W1 31 & MLLW-ORGANIC DEBRIS-SOLVENT/PU LIST (L01)/TC MET (Hg)/CA. & 55440 & 0.20 & 0.27 & 1.66 \\
\hline RL-W141 & MLLW-INORGANIC DEBRIS-TC MET (AS) & 55410 & 10.56 & 16.33 & 38.79 \\
\hline$R L-W 142$ & MLLW-INORGANIC DEBRIS-SOLV/TC MET & 55119 & 12.74 & 39.03 & 205.43 \\
\hline RL-W1 46 & MLLW-ORGANIC DEBRIS-PU LIST/ICR & $s 5440$ & 0.42 & 0.56 & 3.47 \\
\hline RL-W1 47 & MLLW-MERCURY BATTERIES & $\times 7430$ & 0.20 & 0.13 & 0.01 \\
\hline RL-W1 50 & MLLW-INORGANIC DEBRIS-TC METAL $(\mathrm{Hg}\} / \mathrm{CA}$ & s5420 & 0.21 & 0.64 & 3.35 \\
\hline RL-W1 51 & MLLW-INORGANIC DEBRIS-TC ORG/TC METAL/CA & 55420 & 0.20 & 0.61 & 3.22 \\
\hline RL-W1 $5 ?$ & MLLW-ELEMENTAL LEAD-SOLVENT/TC METAL/CA & $x 7219$ & 1.11 & 0.66 & 0.88 \\
\hline RL $-W 171$ & Offsite lab inorganic particulates with metals & s3110 & 5.83 & 0.00 & 0.00 \\
\hline RL-W1 72 & offsite lab inorganic particulates with $\mathrm{Hg}$ & 53110 & 0.86 & 0.00 & 0.00 \\
\hline RL-W1 73 & offsite lab inorganic particulates w/o org, $\mathrm{Hg}$, inet & S3110 & 0.21 & 0.00 & 0.00 \\
\hline RL-W1 75 & Offsite lab metal debris & S5110 & 1.25 & 0.00 & 0.00 \\
\hline RL-W1 76 & Offsite lab organic debris & S5300 & 5.83 & 0.00 & 0.00 \\
\hline RL-W1 79 & Offsite lab elemental lead waste & $x>210$ & 17.07 & 0.00 & 0.00 \\
\hline RL-W183 & Tank Farms inorganic particulates with $\mathrm{Hg}$ & $\$ 3110$ & 0.42 & 0.00 & 0.00 \\
\hline $\mathrm{RL}-\mathrm{W} 184$ & Tank Farms soils contaminated with org t met & $\$ 4200$ & 21.82 & 0.00 & 0.00 \\
\hline$R L-W 185$ & Tank Farms soils contaminated with org & $\$ 4200$ & 261.73 & 0.00 & 0.00 \\
\hline RL-W186 & Tank Farms metal debris & 55110 & 121.84 & 0.00 & 0.00 \\
\hline RL-W1 87 & Tank Farms inorganic non-metal debris & 55120 & 26.56 & 0.00 & 0.00 \\
\hline RL-W1 88 & Tank Farms asbestos debris & S5125 & 126.76 & 0.00 & 0.00 \\
\hline RL-W189 & Tank Earms organic debris & $\$ 5300$ & $1,257.53$ & 0.00 & 0.00 \\
\hline RL-W1 90 & Tank Farms heterogeneous debris & 55400 & 63.20 & 0.00 & 0.00 \\
\hline RL-W1 92 & Tank Farms elemental lead waste & $x>210$ & 0.21 & 0.00 & 0.00 \\
\hline RL-W195 & Tank Farms unspecified forms with $\mathrm{Hg}$ & 49999 & 0.42 & 0.00 & 0.00 \\
\hline RL-W1 98 & Euel fab inorganic particulates with met & $\$ 3110$ & 1.46 & 0.00 & 0.00 \\
\hline RL-W199 & Euel fab heterogeneous debris & $\$ 5400$ & 0.42 & 0.00 & 0.00 \\
\hline$R L-W 200$ & Alkall metal storage debris & $\$ 5110$ & 8.25 & 0.00 & 0.00 \\
\hline RL-W203 & Inorganic particulates with met & $\$ 3110$ & 1.48 & 0.00 & 0.00 \\
\hline RL-W204 & Inorganic particulates with $\mathrm{Hg}$ & $\$ 3110$ & 0.21 & 0.00 & 0.00 \\
\hline RL-W205 & Inorganic particulates without org, $\mathrm{Hg}$, or met & $\$ 3110$ & 1.67 & 0.00 & 0.00 \\
\hline RL-W207 & Soils with Hg & $\$ 4200$ & 0.83 & 0.00 & 0.00 \\
\hline $\mathrm{RL}-\mathrm{W} 208$ & Metal debris & $\$ 5110$ & 16.38 & 0.00 & 0.00 \\
\hline RL-W209 & Asbestos debris & 55125 & 0.21 & 0.00 & 0.00 \\
\hline RL-W210 & Organic debris & 55300 & 47.19 & 0.00 & 0.00 \\
\hline$R L-W 211$ & Heterogeneous debris & 55400 & 49.77 & 0.00 & 0.00 \\
\hline RL-W214 & Unspecified forms with met & U9999 & 0.42 & 0.00 & 0.00 \\
\hline$R L=$ W217 & Elemental lead waste & $x 7210$ & 6.45 & 0.00 & 0.00 \\
\hline RL-W222 & State-only offsite lab absorbed liquids & 53150 & 0.97 & 0.00 & 0.00 \\
\hline RL-W2 24 & State-only inorganic particulates & 53110 & 2.29 & 0.00 & 0.00 \\
\hline$R L-W 225$ & State-only soils & 54200 & 0.53 & 0.00 & 0.00 \\
\hline
\end{tabular}


Table 6. Mixed low-level waste streams by targeted treatment capability (m3) (sheet 3 of 10 ).

\begin{tabular}{|c|c|c|c|c|c|}
\hline WS_1d & Waste Stream Name & $\begin{array}{l}\text { Matrix } \\
\text { Code }\end{array}$ & $\begin{array}{l}\text { Inven- } \\
\text { tory }\end{array}$ & $\begin{array}{l}5 \text { yr pro- } \\
\text { jection }\end{array}$ & $\begin{array}{l}\text { Remaining } \\
\text { life-cycle } \\
\text { projection }\end{array}$ \\
\hline RL $-W 226$ & State-only non-metal debris & $\mathbf{5 5 1 2 0}$ & 98.60 & 0.00 & 0.00 \\
\hline RL $-W 227$ & State-only organic debris & 55300 & 0.21 & 0.00 & 0.00 \\
\hline$R L-W 228$ & State-only heterogeneous debris & 55400 & 37.53 & 0.00 & 0.00 \\
\hline RL-W229 & State-only unspecified waste forms & U9999 & 0.21 & 0.00 & 0.00 \\
\hline $\mathrm{RL}-\mathrm{W} 232$ & Mixed TF metal debris & $\mathbf{5 5 1 1 0}$ & 8.70 & 0.00 & 0.00 \\
\hline$R L-W 233$ & Mixed TF organic debris & S5300 & 43.50 & 0.00 & 0.00 \\
\hline $\mathrm{RL}-\mathrm{W} 235$ & 100-C Reactor Pumphouse debris & $x 7210$ & 16.00 & 0.00 & 0.00 \\
\hline $\mathrm{RL}-\mathrm{W} 447$ & Non-prog MLLW/CH inorg non-met debris & $\mathbf{5 5 1 2 9}$ & 0.00 & 0.00 & 0.60 \\
\hline$R L-W 448$ & Non-prog MLLW/CH org debris & 55390 & 0.00 & 0.00 & 3.14 \\
\hline$R L=W 449$ & Non-prog MLLW/CH het debris & S5490 & 0.00 & 0.00 & 66.73 \\
\hline RL-W4 51 & Non-prog MLLW/CH lead & $x 7219$ & 0.00 & 0.00 & 21.14 \\
\hline RL $-W 452$ & Wastewater fac MLLW/CH salt waste w/ met & 53140 & 0.00 & 55.32 & $1,847.70$ \\
\hline RL-W4 53 & Misc equip MLLW/CH metal debris & 55119 & 0.00 & 0.00 & $4,000.00$ \\
\hline RL-W4 54 & RED reactor MLLW/CH explosive/propellants & $\times 7600$ & 0.00 & 0.26 & 0.00 \\
\hline RL $-W 459$ & Sgl-shl tank retrieval MLLW/CH trorg debris & $\$ 5190$ & 0.00 & 0.00 & 892.00 \\
\hline RL-W4 63 & R\&D reactor MLLW/CH org debris & 55390 & 0.00 & 0.26 & 0.00 \\
\hline$R L-W 464$ & Tank farm upgrade project MLIW/CH pred lnorg debris & 55420 & 0.00 & 189.45 & 206.10 \\
\hline $\mathrm{RL}-\mathrm{W} 465$ & Tank farm upgrade project MLLW/CH org debris & 55390 & 0.00 & 143.14 & 155.72 \\
\hline RL-W542 & Env Rest MLLW/CH metal debris & 55119 & 0.00 & 5.90 & 0.00 \\
\hline RL $-W 543$ & Non-ptog MLLW/CH batteries & $\times 7490$ & 0.00 & 0.00 & 4.49 \\
\hline$R L-W 544$ & Non-prog MLLW/CH elemental mercury & $x 7100$ & 0.00 & 0.00 & 0.60 \\
\hline$R L-W 545$ & Non-prog MLLW/CH explosive/propellants & $\times 7600$ & 0.00 & 0.00 & 0.01 \\
\hline RL-W546 & Chem Engr Lab MLLW/Ch metal debris & $\$ 5119$ & 0.00 & 6.80 & 0.00 \\
\hline RL-W54 9 & Wastewater fac MLLW/CH batteries & $\times 7490$ & 0.00 & 0.74 & 24.64 \\
\hline$R L_{1}-W 550$ & Wastewater fac MLLW/CH explosive/propellants & $\times 7600$ & 0.00 & 0.74 & 24.64 \\
\hline RL-W551 & Wastewater fac MLLW/CH pred inorg debris & 55420 & 0.00 & 5.90 & 197.09 \\
\hline RL-W552 & Wastewater fac MLLW/CH org debris & 55390 & 0.00 & 3.69 & 123.18 \\
\hline RL-W554 & Dbl-shl tank retrieval MLLW/CH pred inorg debris & 55420 & 0.00 & 0.00 & 1.423 .71 \\
\hline R.L-W556 & HLW vit fac MLLW/CH inorg debris & $\$ 5190$ & 0.00 & 0.00 & $5,220.00$ \\
\hline$R L-W 557$ & LLW vit fac MLLW/CH inorg debris & S5190 & 0.00 & 0.00 & $5,120.00$ \\
\hline RL-W558 & Sodium test fac MLLW/CH explosive/propellants & $\times 7600$ & 0.00 & 0.15 & 0.00 \\
\hline RL-W560 & Tank farm vent upgrade project MLLW/CH metal debris & $\$ 5119$ & 0.00 & 20.23 & 0.00 \\
\hline$R L-W 562$ & Tank farm vent upgrade project MLLW/CH lead & $\times 7219$ & 0.00 & 8.38 & 0.00 \\
\hline RL-W564 & Init tank retrieval project MLLW/CH pred inorg debris & $\$ 5420$ & 0.00 & 418.74 & 418.74 \\
\hline$R L-W 579$ & Assay fac MLLW/CH het debris & 55490 & 0.00 & 4.63 & 70.93 \\
\hline RL-W581 & CY95 Ann Arbor CH RCRA/LLW Inorg Part Abs w/o org w/o met ign & $\$ 3113$ & 0.21 & 0.00 & 0.00 \\
\hline RL-W582 & CY95 Ann Arbor CH RCRA/LLW Pred Org Debr w/ org w/o met & $\$ 5440$ & 0.42 & 0.00 & 0.00 \\
\hline RL-W589 & CY95 Argonne E CH St/LLW Inarg Part Abs w/o org w/o met & 53113 & 0.21 & 0.00 & 0.00 \\
\hline RL-W592 & CY95 Argonne E CH RCRA/LLW Inorg Sldg w/o org w/ met & $\$ 3129$ & 0.21 & 0.00 & 0.00 \\
\hline RL-W593 & CY95 Argonne E CH RCRA/LLW Inorg sldg w/o org w/ $\mathrm{Hg}$ & $\$ 3129$ & 2.71 & 0.00 & 0.00 \\
\hline RL-W594 & CY95 Argonne E CH RCRA/PCB/LLW Inorg Sidg w/o org $\mathrm{W} / \mathrm{Hg}$ & $\$ 3129$ & 0.42 & 0.00 & 0.00 \\
\hline$R L-W 596$ & CY95 Argonne E CH RCRA/LLW Soli/Debris w/o org w/ $\mathrm{Hg}$ & 54200 & 2.08 & 0.00 & 0.00 \\
\hline RL-W597 & CY95 Argonne E CH RCRA/LLW Met Debr W/ org w/o met & 55119 & 0.42 & 0.00 & 0.00 \\
\hline RL-W598 & CY95 Argonne E CH RCRA/LLW Met Debr w/o org w/ met & $\mathbf{5} 5119$ & 0.42 & 0.00 & 0.00 \\
\hline RL-W599 & CY95 Argonne E CH RCRA/LLW Met Debr w/o org $w / \mathrm{Hg}$ & 55119 & 0.21 & 0.00 & 0.00 \\
\hline
\end{tabular}


Table 6. Mixed low-level waste streams by targeted treatment capability (m3) (sheet 4 of 10 ).

\begin{tabular}{|c|c|c|c|c|c|}
\hline WS_1d & Waste Stream Name & $\begin{array}{l}\text { Matrix } \\
\text { Code }\end{array}$ & $\begin{array}{c}\text { Inven- } \\
\text { tory }\end{array}$ & $\begin{array}{l}5 \text { yr pro- } \\
\text { jection }\end{array}$ & $\begin{array}{l}\text { Remalning } \\
\text { life-cycle } \\
\text { projection }\end{array}$ \\
\hline RL-W600 & CY95 Argonne E CH RCRA/LLW Org Debr w/ org w/o met & $\mathbf{S} 5390$ & 1.46 & 0.00 & 0.00 \\
\hline RL-W601 & CY95 Argonne E CH RCRA/LLW org Debr w/o org w/ met & $\mathbf{S 5 3 9 0}$ & 0.62 & 0.00 & 0.00 \\
\hline RL-W602 & CY95 Argonne E CH RCRA/LLW org Debr $\mathrm{w} / \mathrm{o}$ org $\mathrm{W} / \mathrm{Hg}$ & $\mathbf{5 5 3 9 0}$ & 1.04 & 0.00 & 0.00 \\
\hline RL-W603 & CY95 Argonne E CH RCRA/LLW Pred Inorg debr w/ org $w /$ met & $\$ 5420$ & 0.21 & 0.00 & 0.00 \\
\hline RL-W604 & CY95 Argonne E CH RCRA/LLW Pred Inorg debr w/ org w/ $\mathrm{Hg}$ cor, rea & 55420 & 0.21 & 0.00 & 0.00 \\
\hline RL-W605 & CY95 Argonne E CH RCRA/LLW Pred Inorg debr w/o org w/ met & 55420 & 0.21 & 0.00 & 0.00 \\
\hline RL-W606 & CY95 Argonne E CH RCRA/LLW Pred Org Debr w/ org w/ $\mathrm{Hg}$ & 55440 & 0.21 & 0.00 & 0.00 \\
\hline RL-W607 & CY95 Argonne E CH RCRA/LLW Unk/oth Debris w/ org w/ thet & 55900 & 0.21 & 0.00 & 0.00 \\
\hline RL-w608 & CY95 Argonne E CH RCRA/LIW Unk/oth Debris w/ org $w / o$ met rea & $\mathbf{S 5 9 0 0}$ & 0.21 & 0.00 & 0.00 \\
\hline RL-W609 & CY95 Argonne E CH RCRA/LIW Unk/oth Debris w/o org w/ met & $\mathbf{S 5 9 0 0}$ & 0.21 & 0.00 & 0.00 \\
\hline RL-W611 & CY95 Argonne E CH RCRA/LLW Lead w/o org w/ met & $\mathrm{X} 7219$ & 3.54 & 0.00 & 0.00 \\
\hline RL-W612 & CY95 Bat Col CH RCRA/LLW Ash w/o org w/ met & S3111 & 0.62 & 0.00 & 0.00 \\
\hline RL-W614 & CY95 Bat Col CH RCRA/LLW Inorg Sldg w/o org w/ met & 53129 & 0.42 & 0.00 & 0.00 \\
\hline RL-W615 & CY95 Bat Col CH RCRA/LLW Inorg Sldg w/O org $\mathrm{w} / \mathrm{Hg}$ & $\mathbf{S 3 1 2 9}$ & 0.21 & 0.00 & 0.00 \\
\hline RL-W616 & CY95 Bat Col CH RCRA/PCB/LIW Inorg sldg w/o org w/ met & $\mathbf{5 3 1 2 9}$ & 0.21 & 0.00 & 0.00 \\
\hline RL-W617 & CY95 Bat Col CH RCRA/PCB/LLW Inorg sldg w/o org w/ Hg & $\mathrm{S} 3129$ & 0.42 & 0.00 & 0.00 \\
\hline RL-W618 & CY95 Bat Col CH RCRA/LLW Sump Drainline Clean-out Soils w/o org w/ Hg & 54200 & 1.04 & 0.00 & 0.00 \\
\hline RL-W619 & CY95 Bat Col CH RCRA/LLW Inorg Homo slds w/o org w/ Hg & $\$ 3190$ & 0.21 & 0.00 & 0.00 \\
\hline RL-W620 & CY95 Bat Col CH RCRA/LLW Soil/Debris w/o org w/ $\mathrm{Hg}$ & 54200 & 0.42 & 0.00 & 0.00 \\
\hline RL-W621 & CY95 Bat Col CH RCRA/LLW Pred Org Debr w/o org w/ Hg & $S 5440$ & 0.62 & 0.00 & 0.00 \\
\hline RL-W622 & CY95 Bat Col CH RCRA/LLW Unk/oth Matrix w/o org w/ Hg & 09999 & 0.21 & 0.00 & 0.00 \\
\hline RL-W625 & CY95 Bat Col CH RCRA/LLW Lead w/o org w/ met & $x 7219$ & 0.21 & 0.00 & 0.00 \\
\hline RL-W629 & CY95 UC Davis CH RCRA/LLW Aque Lab Packs w/o org w/o met ign & $\times 6200$ & 3.75 & 0.00 & 0.00 \\
\hline RL-W630 & CY95 Gen Atomics CH RCRA/LLW Inorg Part Abs w/o org w/ Hg & 53113 & 0.21 & 0.00 & 0.00 \\
\hline RL-W631 & CY95 Gen Atomics CH RCRA/LLW Inorg Sldg w/o org w/ met & 53129 & 3.50 & 0.00 & 0.00 \\
\hline RL-W633 & CY95 Gen Atomics CH RCRA/LLW Inorg Chem w/o org $\mathrm{w} /$ met cor & $\$ 3160$ & 2.71 & 0.00 & 0.00 \\
\hline RL-W635 & CY95 Gen Atomics CH RCRA/LLW Soil/Debris w/o org w/ met & 54200 & 0.21 & 0.00 & 0.00 \\
\hline RL-W636 & CY95 Gen Atomics CH St/LlW Composite Filters w/o org w/o met & 55410 & 1.87 & 0.00 & 0.00 \\
\hline RL-W637 & CY95 Gen Atomics CH RCRA/LLW Unk/oth Solids w/o org w/ met & $\mathrm{S} 9000$ & 0.42 & 0.00 & 0.00 \\
\hline RL-W639 & CY95 Gen Atomics CH RCRA/LLW oth Elmtal Haz Met w/o org w/ met & $\times 7290$ & 0.21 & 0.00 & 0.00 \\
\hline RL-W640 0 & CY95 Fermi Nat Lab CH St/LLW Chloride Salts w/o org w/o met & $\$ 3141$ & 1.25 & 0.00 & 0.00 \\
\hline RL-W642 & CY95 Fermi Nat Lab CH RCRA/LLW Aque Lab Packs w/o org w/o met ign & $\times 6200$ & 0.42 & 0.00 & 0.00 \\
\hline RL-W643 & CY95 Ferm1 Nat Lab CH St/LLW Aque Lab Packs w/o org w/o met & $\times 6200$ & 0.42 & 0.00 & 0.00 \\
\hline$R L-W 644$ & CY95 Ferm1 Nat Lab CH RCRA/LLW Lead w/o org $w /$ met & $\times 7219$ & 0.62 & 0.00 & 0.00 \\
\hline RL-W648 & CY95 Hanford M\&O CH RCRA/LLW Inorg Part Abs w/o org w/ met ign & $\$ 3113$ & 0.21 & 0.00 & 0.00 \\
\hline RL-W649 & CY95 Hanford M\&O CH RCRA/LLW Inorg Part Abs w/o org w/ met cor & 53113 & 0.21 & 0.00 & 0.00 \\
\hline RL-w650 & CY95 Hanford M\&O CH RCRA/LLW Inorg Part Abs w/o org w/ met & 53113 & 0.42 & 0.00 & 0.00 \\
\hline RL-W651 & CY95 Hanford MsO CH RCRA/LLW Inorg Part Abs w/o org w/o met cor & 53113 & 0.42 & 0.00 & 0.00 \\
\hline RL-W652 & CY95 Hanford M\&O CH St/LLW Inorg Part Abs $w / 0$ org $w / 0$ met & 53113 & 0.83 & 0.00 & 0.00 \\
\hline$R L=\$ 655$ & CY95 Hanford M\&O CH RCPR/LLW Inorg Part w/o org w/ met & S3119 & 1.29 & 0.00 & 0.00 \\
\hline $\mathrm{RL}-$ W656 & CY95 Hanford M\&O CH RCRA/LLW Inorg Part w/o org w/o met cor & 53119 & 0.21 & 0.00 & 0.00 \\
\hline$R L-W 65 \theta$ & CY95 Hanford MGO CH RCRA/LLW Inorg Sldg w/o org w/ Hg & S3129 & 0.21 & 0.00 & 0.00 \\
\hline RL-W659 & CY95 Hanford MsO CH St/LLW Sulfate Salts w/o org w/o met & S3142 & 0.21 & 0.00 & 0.00 \\
\hline RL-W660 & CY95 Hanford M6O CH St/LLW Salt Waste w/o org w/o met & 53149 & 0.21 & 0.00 & 0.00 \\
\hline RL-W662 & CY95 Hanford MEO CH St/LLW Soil/Debris w/o org w/o met & 54200 & 0.21 & 0.00 & 0.00 \\
\hline
\end{tabular}


Table 6. Mixed low-level waste streams by targeted treatment capability (m3) (sheet 5 of 10 ).

\begin{tabular}{|c|c|c|c|c|c|c|c|c|c|}
\hline WS_id & Waste & e Stream & Name & & & $\begin{array}{l}\text { Matrix } \\
\text { Code }\end{array}$ & $\begin{array}{l}\text { Inven- } \\
\text { tory }\end{array}$ & $\begin{array}{l}5 \text { yr pro- } \\
\text { jection }\end{array}$ & $\begin{array}{l}\text { Remaining } \\
\text { life-cycle } \\
\text { projection }\end{array}$ \\
\hline $\mathrm{RL}-\mathrm{W} 663$ & CY95 & Hanford & $\mathrm{M} \& \mathrm{O} \mathrm{CH}$ & RCRA/LLW & Met Debr w/ org w/o met & 55119 & 6.48 & 0.00 & 0.00 \\
\hline RL-W664 & CY95 & Hanford & $\mathrm{M} 6 \mathrm{O} \mathrm{CH}$ & RCRA/LLW & Met Debr w/o org $w /$ inet & 55119 & 11.37 & 0.00 & 0.00 \\
\hline RL-W665 & CY95 & Han ford & $\mathrm{M} \& \mathrm{O} \mathrm{CH}$ & RCRA/LLW & Met Debr w/o org w/o met lgn, cor, rea & 55119 & B. 41 & 0.00 & 0.00 \\
\hline RL-W666 & CY95 & Hanford & $\mathrm{MrO} \mathrm{CH}$ & RCRA/LIW & Inorg Non-met Debr w/ org w/ met & 55129 & 2.53 & 0.00 & 0.00 \\
\hline RL-W667 & CY95 & Hanford & $\mathrm{M} \notin \mathrm{O} \mathrm{CH}$ & RCRA/LIW & Inorg Debr w/ org w/ met & 55190 & 0.21 & 0.00 & 0.00 \\
\hline RL-W668 & CY95 & Hanford & $\mathrm{MGO} \mathrm{CH}$ & RCRA/LLW & Org Debr w/ org w/ met cor & $\$ 5390$ & 0.21 & 0.00 & 0.00 \\
\hline RL-W669 & CY95 & Hanford & $\mathrm{M \& O} \mathrm{CH}$ & RCRA/LIW & Org Debr w/ org w/ met & S5390 & 5.02 & 0.00 & 0.00 \\
\hline RL-W670 & CY95 & Hanford & $\mathrm{M \& O} \mathrm{CH}$ & RCRA/LLW & Org Debr w/ org w/o met cor & $\$ 5390$ & 0.21 & 0.00 & 0.00 \\
\hline RL-W67I & CY95 & Hanford & $\mathrm{MEO} \mathrm{CH}$ & RCRA/LLW & Org Debr w/ org w/o met & $\$ 5390$ & 36.20 & 0.00 & 0.00 \\
\hline RL-W672 & CY95 & Hanford & $\mathrm{MrO} \mathrm{CH}$ & RCRA/LLW & Org Debr w/o org w/ met & 55390 & 1.87 & 0.00 & 0.00 \\
\hline RL-W673 & CY95 & Hanford & $\mathrm{MGO} \mathrm{CH}$ & RCRA/LIW & Org Debr w/o org $w / H g$ & 55390 & 0.42 & 0.00 & 0.00 \\
\hline RL-W674 & CY95 & Han ford & $\mathrm{MGO} \mathrm{CH}$ & RCRA/LLW & org Debr w/o org w/o met 1 gn & 55390 & 39.30 & 0.00 & 0.00 \\
\hline RL-W675 & CY95 & Han ford & MsO CH & St/LLW Or & Ig Debr w/o org w/o thet & 55390 & 13.51 & 0.00 & 0.00 \\
\hline RL-W676 & CY95 & Han ford & $\mathrm{M} 6 \mathrm{O} \mathrm{CH}$ & RCRA/LI.W & Composite Filters w/ org w/ met & 55410 & 0.21 & 0.00 & 0.00 \\
\hline RL-W677 & CY95 & Hanford & $\mathrm{M} 60 \mathrm{CH}$ & RCRA/LLW & Composite Filters w/ org w/o met & 55410 & 15.63 & 0.00 & 0.00 \\
\hline RE-W678 & CY95 & Hanford & $\mathrm{M} \& \mathrm{OCH}$ & RCPA/LLW & Composite Filters w/o org w/ met & 55410 & 3.01 & 0.00 & 0.00 \\
\hline RL-W679 & CY95 & Hanford & M\&O CH & St/LLW Co & omposite Filters w/o org w/o met & 55410 & 1.15 & 0.00 & 0.00 \\
\hline RL-W680 & CY95 & Hanford & $\mathrm{MEO} \mathrm{CH}$ & RCRA/LLW & Pred Inorg debr w/ org w/ met & $\$ 5420$ & 15.54 & 0.00 & 0.00 \\
\hline RL-W681 & CY95 & Hanford & M\&O CH & RCRA/LLW & Pred Inorg debr $w /$ org $w / 0$ met & $\$ 5420$ & 46.26 & 0.00 & 0.00 \\
\hline RL-W683 & CY95 & Hanford & $\mathrm{MSO} \mathrm{CH}$ & RCRA/LLW & Pred Org Debr w/ org w/ met & 55440 & 16.43 & 0.00 & 0.00 \\
\hline RL-W684 & CY95 & Hanford & $\mathrm{M} \& \mathrm{OCH}$ & RCRA/LLW & Pred Org Debr w/ org w/o met & $\$ 5440$ & 19.73 & 0.00 & 0.00 \\
\hline RL $=$ W685 & CY95 & Hanford & $\mathrm{M} \& \mathrm{CH}$ & RCRA/LLW & Pred org Debr w/o org w/ met cor & 55440 & 0.21 & 0.00 & 0.00 \\
\hline RL-W686 & CY95 & Hanford & $\mathrm{MEO} \mathrm{CH}$ & RCRA/LLW & Pred Org Debr w/o org w/ met & $S 5440$ & 0.62 & 0.00 & 0.00 \\
\hline RL-W687 & CY95 & Hanford & $\mathrm{MEO} \mathrm{CH}$ & RCRA/LLW & Pred Org Debr w/O org w/ Hg cor & 55440 & 0.21 & 0.00 & 0.00 \\
\hline$R L-W 688$ & CY95 & Hanford & $\mathrm{MEO} \mathrm{CH}$ & RCRA/LLW & Pred Org Debr w/O org w/ Hg & 55440 & 0.42 & 0.00 & 0.00 \\
\hline$R L-W 689$ & CY95 & Hanford & $\mathrm{MEO} \mathrm{CH}$ & RCRA/LLW & Pred Org Debr w/o org w/o met ign & 55440 & 0.21 & 0.00 & 0.00 \\
\hline RL-W690 & CY95 & Hanford & $\mathrm{MEO} \mathrm{CH}$ & RCRA/LLW & Pred Org Debr w/o org w/o met cor & 55440 & 0.42 & 0.00 & 0.00 \\
\hline$R L-W 691$ & CY95 & Hanford & $\mathrm{MrO} \mathrm{CH}$ & St/LLW Pr & red Org Debr w/o org w/o met & 55440 & 12.02 & 0.00 & 0.00 \\
\hline RL-W692 & CY95 & Hanford & $\mathrm{M} 5 \mathrm{O} \mathrm{CH}$ & RCRA/LLW & Unk/oth Debris w/ org w/ met & $\$ 5900$ & 25.52 & 0.00 & 0.00 \\
\hline RL - W693 & CY95 & Hanford & MaO CH & RCRA/ LLW & Unk/oth Debris w/ org w/o met & $\$ 5900$ & 1.48 & 0.00 & 0.00 \\
\hline RL-W694 & CY95 & Hanford & $\mathrm{MGO} \mathrm{CH}$ & RCRA/LLW & Unk/oth Debris w/o org w/ met. & 55900 & 0.42 & 0.00 & 0.00 \\
\hline RL-W695 & CY95 & M\&O Lab & CH RCRA & A/LLW Unk/ & /oth Debris w/ Be w/o org w/ $\mathrm{Hg}$ & $\$ 5900$ & 3.12 & 0.00 & 0.00 \\
\hline RL-W697 & CY95 & Hanford & $\mathrm{M \& O} \mathrm{CH}$ & RCRA/LLW & Unk/oth Solids w/o org w/ met & $\$ 9000$ & 0.21 & 0.00 & 0.00 \\
\hline RL-W700 & CY95 & Henford & $\mathrm{MaOCH}$ & St/LLW Un & nk/oth Matrix w/o org w/o met & 09999 & 0.21 & 0.00 & 0.00 \\
\hline RL-W706 & CY95 & Hanford & MGO CH & RCRA/LLW & Aque Lab Packs w/o org w/ met & $x 6200$ & 0.21 & 0.00 & 0.00 \\
\hline$R L-W 707$ & CY95 & Hanford & $\mathrm{MaO} \mathrm{CH}$. & RCRA/LLW & Aque Lab Packs w/o org $\mathrm{w} / \mathrm{Hg}$ & $\mathrm{x} 6200$ & 0.21 & 0.00 & 0.00 \\
\hline$R L-W 708$ & CY95 & Hanford & $\mathrm{MLO} \mathrm{CH}$ & RCRA/LLW & Aque Lab Packs w/o org w/o met ign & $\mathrm{x} 6200$ & 0.62 & 0.00 & 0.00 \\
\hline RL-W709 & CY95 & Hanford & $\mathrm{M} \& \mathrm{O} \mathrm{CH}$ & RCRA/LLW & Aque Lab Packs w/o org w/o met cor & $\mathrm{X} 6200$ & 0.42 & 0.00 & 0.00 \\
\hline RL-W7 10 & CY95 & Hanford & $\mathrm{M} \times \mathrm{OCH}$ & St/LLW Aq & que Lab Packs w/o org w/o met & $\times 6200$ & 0.42 & 0.00 & 0.00 \\
\hline RL-W711 & CY95 & Hanford & $\mathrm{M \& O} \mathrm{CH}$ & RCRA/LLW & Lead w/ org w/ met & $\times 7219$ & 8.72 & 0.00 & 0.00 \\
\hline RL-W712 & CY95 & Hanford & $\mathrm{MGO} \mathrm{CH}$ & RCRA/LEW & Lead w/o org w/ met & $x 7219$ & 4.58 & 0.00 & 0.00 \\
\hline RL-W713 & CY95 & Hanford & M६् CH & RCRA/LLW & Oth Elmtal Haz Met w/o org w/ met & $\times 7290$ & 0.42 & 0.00 & 0.00 \\
\hline RL-W714 & CY95 & Hanford & $\mathrm{MGO} \quad \mathrm{CH}$ & RCRA/LLW & Unk batteries $w / 0$ org $w / \mathrm{Hg}$ & $x 7490$ & 0.21 & 0.00 & 0.00 \\
\hline RL-W715 & CY95 & Hanford & $\mathrm{MsO} \mathrm{CH}$ & RCRA/LLW & Reactive Metals w/o org w/o met ign, cor, rea & $x 7590$ & 5.43 & 0.00 & 0.00 \\
\hline RL-W722 & CY95 & $\mathrm{Pac} \mathrm{N} \mathrm{Na}$ & at Lab C & CH RCRA/LL & LW Org Debr w/ org w/o met & 55390 & 2.86 & 0.00 & 0.00 \\
\hline
\end{tabular}


Table 6. Mixed low-level waste streams by targeted treatment capability (m3) (sheet 6 of 10 ).

\begin{tabular}{|c|c|c|c|c|c|}
\hline WS_id & Waste Stream Name & $\begin{array}{l}\text { Matrix } \\
\text { Code }\end{array}$ & $\begin{array}{l}\text { Inven- } \\
\text { tory }\end{array}$ & $\begin{array}{l}5 \text { yr pro- } \\
\text { jection }\end{array}$ & $\begin{array}{l}\text { Remaining } \\
\text { life-cycle } \\
\text { projection }\end{array}$ \\
\hline$R L-W 723$ & CY95 Pac N Nat Lab CH RCRA/LLW org Debr w/o org w/ met & $\$ 5390$ & 0.32 & 0.00 & 0.00 \\
\hline RL-W724 & CY95 Pac N Nat Lab CH RCRA/LLW Composite Filters $\mathrm{w} / 0$ org $\mathrm{w} /$ met & 55410 & 1.28 & 0.00 & 0.00 \\
\hline$R L-W 725$ & CY95 Pac N Nat Lab CH St/LLW Composite Filters w/o org w/o met & 55410 & 2.88 & 0.00 & 0.00 \\
\hline RL-W726 & CY95 Pac N Nat Lab CH RCRA/LLW Pred Org Debr w/o org w/o met cor & S5440 & 0.21 & 0.00 & 0.00 \\
\hline RL-W727 & CY95 PaC N Nat Lab CH RCRA/LLW Unk/oth Debris w/ org w/o met & $\mathbf{5 5 9 0 0}$ & 0.21 & 0.00 & 0.00 \\
\hline RL $-W 728$ & CY95 Pac N Nat Lab CH RCRA/LLW Unk/Oth Matrix w/o org w/ met & U9999 & 2.29 & 0.00 & 0.00 \\
\hline$R L-W 729$ & CY95 Pac N Nat Lab CH RCRA/LLW Unk/Oth Matrix w/o org w/ $\mathrm{Hg}$ & U9999 & 4.20 & 0.00 & 0.00 \\
\hline$R L-W 730$ & CY95 Pac N Nat Lab CH St/LLW Unk/Oth Matrix w/o org w/o met & U9999 & 0.21 & 0.00 & 0.00 \\
\hline RL-W7 36 & CY95 Pac N Nat Lab CH RCRA/LLW Aque Lab Packs w/o org w/ met cor & $\mathrm{x} 6200$ & 4.37 & 0.00 & 0.00 \\
\hline RL-W737 & CY95 Pac $N$ Nat Lab CH RCRA/LLW Aque Lab Packs $w / 0$ org $w /$ met & $\mathrm{x} 6200$ & 0.62 & 0.00 & 0.00 \\
\hline RL-W738 & CY95 Pac N Nat Lab CH RCRA/LLW Aque Lab Packs w/o org w/o met ign & $\mathrm{x} 6200$ & 1.25 & 0.00 & 0.00 \\
\hline RL-W7 39 & CY95 Pac N Nat Lab CH RCRA/LLW Aque Lab Packs w/o org w/o met ign, cor & $\mathrm{x} 6200$ & 0.42 & 0.00 & 0.00 \\
\hline RL-W740 & CY95 Pac N Nat Lab CH St/LLW Aque Lab Packs w/o org w/o met & $\mathrm{x} 6200$ & 0.62 & 0.00 & 0.00 \\
\hline RL-W74 1 & CY95 Pac N Nat Lab CH RCRA/LLW Lead w/o org $w /$ met & X7219 & 6.55 & 0.00 & 0.00 \\
\hline RL-W753 & CY $96 \mathrm{CH}$-LLW/RCRA liq, part, soils, labpacks, $\mathrm{PCB}{ }^{\circ} \mathrm{s}$ w t met tno $\mathrm{Hg}$ ) (thru $6 / 30$ ) & s3900 & 11.28 & 0.00 & 0.00 \\
\hline RL-W754 & CY 96 CH-LLW/RCRA debris (thru 6/30) & 55900 & 100.06 & 0.00 & 0.00 \\
\hline$R L-W 755$ & CY $96 \mathrm{CH}-L L W / R C R A$ elemental lead (thru $6 / 30$ ) & $x 7290$ & 4.94 & 0.00 & 0.00 \\
\hline RL-W758 & Non-characterized 1987 to 1993 LLMW & S9000 & 20.00 & 0.00 & 0.00 \\
\hline Non-thermal & I treatment total & & $7,201.00$ & $2,233.83$ & $26,243.24$ \\
\hline RL-W017 & IGNITABLE LIQUIDS - TOC $>10 \%$ & $\mathrm{x} 6100$ & 0.42 & 0.24 & 0.40 \\
\hline RL-W021 & TC METAL SLUDGES/DRY PARTICULATE (CA, HG) & 53114 & 0.21 & 0.08 & 0.54 \\
\hline RL-W027 & TC METAL INORGANIC SOLID DEBRIS (HG, PCB) & $\mathbf{S 5 4 2 0}$ & 9.61 & 29.44 & 154.94 \\
\hline RL-W029 & TC METAL ORGANIC SOLID DEBRIS & 53114 & 35.02 & 13.38 & 89.32 \\
\hline RL-Wo 31 & TC METAL ORGANIC SOLID DEBRIS (CA,AS) & $\$ 3114$ & 1.05 & 0.40 & 2.68 \\
\hline RL-W039 & ORGANIC RMW PCB LIQUIDS 50-500 PPM (CA) & $\mathrm{x} 6100$ & 11.34 & 5.59 & 10.82 \\
\hline RL-W040 & ORGANIC RMW PCB LIQUIDS $>500$ PPM (CA) & $\mathrm{x} 6100$ & 22.05 & 12.81 & 21.05 \\
\hline RL-W042 & APPENDIX IV LABPACKS & $\mathrm{x} 6900$ & 7.31 & 4.25 & 6.98 \\
\hline RL-W04 3 & APPENDIX IV LABPACKS $(C A)$ & $\mathrm{x} 6900$ & 12.25 & 7.12 & 11.69 \\
\hline RL-W044 & APPENDIX IV LABPACKS $\{\mathrm{CA}, \mathrm{HG}\}$ & $\mathrm{x} 6900$ & 0.68 & 0.40 & 0.65 \\
\hline RL-W04 5 & APPENDIX IV LABPACKS (CA, PCB) & $\mathrm{x} 6900$ & 0.21 & 0.12 & 0.20 \\
\hline RL-W045 & APPENDIX $V$ LABPACKS & $\mathrm{x} 6100$ & 11.29 & 6.56 & 10.78 \\
\hline RL-W049 & SOLVENT SOILS & $\$ 4200$ & 39.68 & 26.16 & 39.41 \\
\hline RL-W050 & SOLVENT SOILS (CA) & $\$ 4200$ & 1.24 & 0.82 & 1.24 \\
\hline RL-W051 & SOLVENT/TC METAL SOILS (HG) & $\$ 4100$ & 0.21 & 0.14 & 0.21 \\
\hline RL-W052 & SOLVENT SLUDGES/DRY PARTICULATE & 53114 & 3.09 & 1.18 & 7.87 \\
\hline RL-W053 & SOLVENT/TC METAL SLUDGES/DRY PART. (CA) & 53114 & 1.49 & 0.57 & 3.79 \\
\hline RL-W054 & SOLVENT TC METAL SLUDGES/DRY PART (HG) & 53129 & 10.62 & 4.05 & 27.09 \\
\hline RL-W061 & SOLVENT/TC METAL ORG. SOLID DEBRIS CA, Q & 53114 & 1.05 & 0.40 & 2.68 \\
\hline RL-W063 & SOLVENT APPENDIX IV LABPACKS & $\mathrm{x} 6900$ & 3.51 & 2.04 & 3.35 \\
\hline RL-W064 & SOLVENT APPENDIX IV LABPACKS (CA) & $\times 6900$ & 36.59 & 21.26 & 34.92 \\
\hline RL-W065 & SOLVENT APPENDIX $\vee$ LABPACKS & $\times 6900$ & 29.89 & 17.37 & 28.53 \\
\hline RL-W066 & SOLVENT APPENDIX $\vee$ LABPACKS (CA) & $\times 6900$ & 9.68 & 5.63 & 9.24 \\
\hline RL-W067 & SOLVENT APPENDIX V LABPACKS $(C A, Q)$ & $\times 6100$ & 0.21 & 0.12 & 0.20 \\
\hline $\mathrm{RL}-\mathrm{WO} 87$ & SOLVENT SLUDGES/DRY PARTICULATE (CA) & 53119 & 7.14 & 2.73 & 18.22 \\
\hline
\end{tabular}


Table 6. Mixed low-level waste streams by targeted treatment capability (m3) (sheet 7 of 10).

\begin{tabular}{|c|c|c|c|c|c|}
\hline WS id & Waste Stream Name & $\begin{array}{l}\text { Matrix } \\
\text { Code }\end{array}$ & $\begin{array}{l}\text { Inven- } \\
\text { tory }\end{array}$ & $\begin{array}{l}5 \text { yr pro- } \\
\text { Jection }\end{array}$ & $\begin{array}{l}\text { Remaining } \\
\text { life-cycle } \\
\text { projection }\end{array}$ \\
\hline RL-W090 & TC ORGANIC SLUDGES/DRY PARTICULATE & 53119 & 17.43 & 6.66 & 44.44 \\
\hline RL-W092 & WA REGULATED IGNITABLE LIQ. - TOC>108 & $x 6100$ & 0.03 & 0.02 & 0.03 \\
\hline RL-W099 & WA REGULATED ACIDIC AQUEOUS CORROSIVE & $\mathrm{x} 6200$ & 0.63 & 0.37 & 0.60 \\
\hline RL-W100 & WA REGULATED LABPACKS & $\times 6900$ & 33.77 & 19.62 & 32.23 \\
\hline RL-W10B & MLLW-SLUDGE/PARTICULATE-TC ORG/PCB2 & 53119 & 5.60 & 2.14 & 14.28 \\
\hline RL $-W 110$ & MLLW-ORGANIC DEBRIS-TC ORG/PCB 1 & 55390 & 3.40 & 4.51 & 28.25 \\
\hline RL-W114 & MLLW-SLUDGE/PART ICULATE-SOLVENT /TC MET & 53119 & 0.84 & 0.32 & 2.13 \\
\hline RL-W119 & MLLW-SOIL-PU LIST (LIO)/TC MET (AS) & 54200 & 0.20 & 0.13 & 0.20 \\
\hline RL-W122 & MLLW-SOIL-SOLVENT/TC MET & 54200 & 10.70 & 7.06 & 10.63 \\
\hline RL $-W 123$ & MLLW-SOIL-TC ORG & 54200 & 1.24 & 0.82 & 1.23 \\
\hline RL-W124 & MLLW-SLUDGE/PARTICULATE-SOLVENT/TC MET $(\mathrm{Hg}) / \mathrm{CA}$ & 53119 & 1.26 & 0.48 & 3.21 \\
\hline $\mathrm{RL}-W 125$ & MLLW-ORGANIC DEBRIS-SOLVENT/PCB $2 / T C \mathrm{MET} / \mathrm{CA}$ & $\$ 5440$ & 1.05 & 1.39 & 8.72 \\
\hline$R L-W 130$ & MLLW-APPENDIX $\vee$ LABPACKS-CA & $\mathrm{x} 6900$ & 2.85 & 1.66 & 2.72 \\
\hline $\mathrm{RL}-\mathrm{W} 132$ & MLLW-APPENDIX IV LABPACKS $-\mathrm{Hg}$ & $x 6900$ & 1.63 & 0.95 & 1.55 \\
\hline RL-W139 & WA REG. TC METAL ORG. SOLID DEBRIS & 55490 & 48.90 & 75.63 & 179.64 \\
\hline $\mathrm{RI}-\mathrm{W} 140$ & MLLW-SOIL-SOLV/PU LIST (L01, L02, LO3) & 54200 & 67.87 & 44.75 & 67.42 \\
\hline$R L-W 143$ & MLLW-SLUDGE/PARTICULATE-SOLVENT (As) & S3114 & 0.32 & 0.12 & 0.82 \\
\hline RL $-W 144$ & MLLW-APPENDIX IV LABPACKS-SOLVENT $(\mathrm{Hg})$ & $x 6900$ & 0.21 & 0.12 & 0.20 \\
\hline$R L-W 145$ & MLLW-INORGANIC DEBRIS-TC ORGANIC & S3114 & 0.21 & 0.08 & 0.53 \\
\hline $\mathrm{RL}-\mathrm{W1} 48$ & MILW-SLUDGE/FARTICULATE-ICR/CA & 53119 & 0.20 & 0.08 & 0.51 \\
\hline RL-W1 49 & MLEW-SLUDGE/FART-TC ORGANIC/TC METAL/CA & $\$ 3119$ & 0.21 & 0.08 & 0.53 \\
\hline RL $-W 153$ & MLLW-SOIL-SOLVENT/TC METAL/CA & $\$ 4100$ & 0.21 & 0.14 & 0.21 \\
\hline RL-W1 70 & Offsite lab inorganic particulates with org & $\$ 3110$ & 1.46 & 0.00 & 0.00 \\
\hline RL-W1 74 & Offsite lab contaminated soils & S4200 & 5.83 & 0.00 & 0.00 \\
\hline RL-W177 & Offsite lab organic lab packs & $\mathrm{x} 6100$ & 40.01 & 0.00 & 0.00 \\
\hline RL-W1 78 & offsite lab other lab packs & $\mathrm{x} 6900$ & 2.27 & 0.00 & 0.00 \\
\hline $\mathrm{RL}-\mathrm{W} 180$ & Tank Farms oil waste & L2200 & 0.21 & 0.00 & 0.00 \\
\hline $\mathrm{RL}-\mathrm{W} 181$ & Tank Farms inorganic particulates with org, met & S3110 & 0.21 & 0.00 & 0.00 \\
\hline RL-W1 82 & Tank Farms inorganic particulates with org & S3110 & 19.33 & 0.00 & 0.00 \\
\hline RL-W1 91 & Tank Earms lab packs & $x 6900$ & 0.42 & 0.00 & 0.00 \\
\hline RL-W193 & Tank Earms unspecified forms with org \& met & U9999 & 0.42 & 0.00 & 0.00 \\
\hline RL-W1 94 & Tank Farms unspecified forms with org & U9999 & 12.57 & 0.00 & 0.00 \\
\hline RL-W196 & Fuel fab inorganic particulates with org a met & 53110 & 4.07 & 0.00 & 0.00 \\
\hline RL-W197 & Fuel fab inorganic particulates with org & $\$ 3110$ & 7.50 & 0.00 & 0.00 \\
\hline RL-W201 & Inorganic particulates with org \& met & $\$ 3110$ & 1.25 & 0.00 & 0.00 \\
\hline $\mathrm{RL}-\mathrm{W} 2 \mathrm{O} 2$ & Inorganic particulates with org & 53110 & 2.63 & 0.00 & 0.00 \\
\hline $\mathrm{RL}-\mathrm{W} 206$ & Solls with organies & $\$ 4200$ & 1.04 & 0.00 & 0.00 \\
\hline $\mathrm{RL}-\mathrm{W} 212$ & Unspecified forms with org \& met & U9999 & 0.42 & 0.00 & 0.00 \\
\hline RL-W213 & Unspecified forms with org & U9999 & 4.19 & 0.00 & 0.00 \\
\hline $\mathrm{RL}-\mathrm{W} 215$ & Organic lab packs & $\mathrm{x} 6100$ & 17.49 & 0.00 & 0.00 \\
\hline $\mathrm{RL}-W 216$ & Other lab packs & $\times 6900$ & 6.87 & 0.00 & 0.00 \\
\hline $\mathrm{RL}-\mathrm{W} 218$ & Tank Farm organic debris with org \& PCA's & $\mathbf{5} 5300$ & 0.83 & 0.00 & 0.00 \\
\hline $\mathrm{R} L-\mathrm{W} 219$ & Solls with org, met, \& $\mathrm{PCB}^{\circ} \mathrm{s}$ & 54200 & 0.64 & 0.00 & 0.00 \\
\hline RL-W220 & Organic debris with PCB's & S5300 & 1.46 & 0.00 & 0.00 \\
\hline $\mathrm{RL}-\mathrm{W} 221$ & Organic labpacks with PCB's & $\times 6100$ & 0.42 & 0.00 & 0.00 \\
\hline
\end{tabular}


Table 6. Mixed low-level waste streams by targeted treatment capability (m3) (sheet 8 of 10 ).

\begin{tabular}{|c|c|c|c|c|c|}
\hline WS_id & Waste Stream Name & $\begin{array}{l}\text { Matrix } \\
\text { Code }\end{array}$ & $\begin{array}{l}\text { Inven- } \\
\text { tory }\end{array}$ & $\begin{array}{r}5 \text { yr pro- } \\
\text { jection }\end{array}$ & $\begin{array}{l}\text { Remaining } \\
\text { life-cycle } \\
\text { projection }\end{array}$ \\
\hline RL-W223 & State-only offsite lab non-organic lab packs & $\times 6900$ & B. 54 & 0.00 & 0.00 \\
\hline RL-W230 & State-only lab packs & $\times 6900$ & 2.91 & 0.00 & 0.00 \\
\hline RL-W231 & Solls from the Mixed TF & 54200 & 31.86 & 0.00 & 0.00 \\
\hline RL-W445 & Env Rest MLLW/CH soils & 54200 & 0.00 & 4.27 & 0.00 \\
\hline RL-W446 & Non-prog MLLW/CH lab packs & $\times 6100$ & 0.00 & 0.00 & 92.30 \\
\hline RL-W450 & Non-prog MLLW/CH soils & 54200 & 0.00 & 0.00 & 1.49 \\
\hline RL-W4 66 & Tank farm upgrade project MLLW/CH soils & $\$ 4200$ & 0.00 & 88.41 & 96.18 \\
\hline RL -W547 & Chern Engr Lab MLLW/CH lab packs & $\times 6100$ & 0.00 & 13.20 & 0.00 \\
\hline RL $=$ W5 48 & Post-irridiation lab MLLW/CH inorg homo solids & $\$ 3190$ & 0.00 & 1.03 & 0.00 \\
\hline RL-W553 & Wastewater fac MLLW/CH lab packs & $\mathrm{x} 6100$ & 0.00 & 0.74 & 24.64 \\
\hline RL $-W 555$ & Dbl-shl tank retrieval MLLW/CH soils & $\$ 4200$ & 0.00 & 0.00 & 54.61 \\
\hline RL-W559 & Tank farm vent upgrade project MLLW/CH inorg homo solids & $\$ 3190$ & 0.00 & 12.58 & 0.00 \\
\hline RL-W561 & Tank farm vent upgrade project MLLW/CH soils & 54200 & 0.00 & 0.73 & 0.00 \\
\hline RL-W563 & Tank cross site-line project $\mathrm{MLLW} / \mathrm{CH}$ soils & 54200 & 0.00 & 5.14 & 0.00 \\
\hline RL-W565 & Init tank retrieval project $\mathrm{MLLW} / \mathrm{CH}$ soils & 54200 & 0.00 & 16.06 & 16.06 \\
\hline RL-W576 & Wastewater fac MLLW/CH salt waste w/ orgtmet & $\$ 3140$ & 0.00 & 6.64 & 221.72 \\
\hline RL-W580 & CY95 Ann Arbor CH RCRA/LLW Inorg Part Abs w/ org w/o met & 53113 & 0.21 & 0.00 & 0.00 \\
\hline RL-W583 & CY95 Ann Asbor CH RCRA/LLW Unk/oth Matrix w/ org w/o met & U9999 & 0.62 & 0.00 & 0.00 \\
\hline RL-W584 & CY95 Ann Arbor CH RCRA/LLW Org Lab Packs w/ org w/ met & $\times 6100$ & 0.21 & 0.00 & 0.00 \\
\hline RL-W585 & CY95 Ann Arbor CH RCRA/LLW Org Lab Packs $w /$ org w/o thet ign & $\times 6100$ & 0.21 & 0.00 & 0.00 \\
\hline RL $-\$ 586$ & CY95 Ann Arbor CH RCRA/LLW Org Lab Packs w/ org w/o net & $\mathrm{x} 6100$ & 0.42 & 0.00 & 0.00 \\
\hline RL-W587 & CY95 Argonne E CH RCRA/LLW Sandblasting Media w/ org w/o met & $\mathrm{s} 3112$ & 0.21 & 0.00 & 0.00 \\
\hline RL-W588 & CY95 Argonne E CH RCRA/LLW Inorg Part Abs w/ org w/o met & 53113 & 2.50 & 0.00 & 0.00 \\
\hline RL-W590 & CY95 Argonne E CH RCRA/LLW Inorg Sldg w/ org $\mathrm{w} / \mathrm{met}$ & 53129 & 1.46 & 0.00 & 0.00 \\
\hline RL-W591 & CY95 Argonne E CH RCRA/LLW Inorg Sldg w/ org w/o met & 53129 & 0.62 & 0.00 & 0.00 \\
\hline RL-W595 & CY95 Argonne E CH RCRA/LLW Paint Sludges $w /$ org $w / o$ met & 53132 & 1.46 & 0.00 & 0.00 \\
\hline RL-W610 & CY95 Argonne E CH RCRA/LLW Unk/oth Matrix w/ org w/o met & U9999 & 1.04 & 0.00 & 0.00 \\
\hline RL-W613 & CY95 Bat Col CH RCRA/LLW Inorg sldg $w /$ org w/o met & 53129 & 0.21 & 0.00 & 0.00 \\
\hline RL-W623 & CY95 Bat Col CH RCRA/LLW org Lab Packs w/ org w/ met & $\times 6100$ & 0.42 & 0.00 & 0.00 \\
\hline RL-W624 & CY95 Bat Col CH RCRA/LLW Org Lab Packs w/ org w/o met & $\mathrm{K} 6100$ & 2.29 & 0.00 & 0.00 \\
\hline RL-W626 & CY95 Brookhaven NL CH RCRA/LIW Inorg sldg $w /$ org $w /$ met & 53129 & 22.69 & 0.00 & 0.00 \\
\hline RL-W627 & CY95 UC Davis CH RCRA/LLw Org Lab Packs w/ org w/o met ign & $\mathrm{x} 6100$ & 0.21 & 0.00 & 0.00 \\
\hline RL-W628 & CY95 UC Davis CH RCRA/LLW org Lab Packs w/ org w/o met ign, cor & $\mathrm{x} 6100$ & 2.50 & 0.00 & 0.00 \\
\hline RL-W632 & CY95 Gen Atomics CH RCRA/LLW Paint Sludges $\mathrm{w} /$ org w/o met & 53132 & 0.21 & 0.00 & 0.00 \\
\hline RL-W634 & CY95 Gen Atomics CH RCRA/LLW Soli/Debris w/ org w/o met & 59200 & 1.67 & 0.00 & 0.00 \\
\hline $\mathrm{RL}-\mathrm{W} 638$ & CY95 Gen Atomics CH RCRA/LLW Org Lab Packs w/ org w/ met ign & $\mathrm{x} 6100$ & 1.67 & 0.00 & 0.00 \\
\hline RL-W64 1 & CY95 Fermi Nat Lab CH RCRA/LLW org Lab Packs $w /$ org $w / 0$ met & $\times 6100$ & 0.21 & 0.00 & 0.00 \\
\hline RL-W64 5 & CY95 Hanford MsO CH RCRA/LLW oth Org Liq w/O org $\mathrm{w} / \mathrm{met}$ & L2290 & 0.83 & 0.00 & 0.00 \\
\hline RL-W64 6 & CY95 Hanford MGO CH RCRA/LLW Inorg Part Abs $w /$ org $w /$ met & 53113 & 0.21 & 0.00 & 0.00 \\
\hline RL-W64 7 & CY95 Hanford M\&O CH RCRA/LLW. I norg Part Abs w/ org w/o met & 53113 & 0.83 & 0.00 & 0.00 \\
\hline RL-W653 & CY95 Hanford MsO CH RCRA/LLW Inorg Part w/ org w/o met cot & 53119 & 0.32 & 0.00 & 0.00 \\
\hline$R L-W 654$ & CY95 Hanford MaO CH RCPA/LLW Inorg Part W/ org w/o met & S3119 & 0.32 & 0.00 & 0.00 \\
\hline RL-W657 & CY95 Hanford MsO CH RCRA/LLW Inorg Sldg w/ org w/ met & 53129 & 0.42 & 0.00 & 0.00 \\
\hline RL-W661 & CY95 Hanford MbO CH RCRA/LLW SOll/Debris W/ org w/o met & 54200 & 11.70 & 0.00 & 0.00 \\
\hline RL-W682 & CY95 Hanford MsO CH RCRA/PCB/LLW Pred Inorg debr w/o org w/ met & 55420 & 12.10 & 0.00 & 0.00 \\
\hline
\end{tabular}


Table 6. Mixed low-level waste streams by targeted treatment capability (m3) (sheet 9 of 10).

\begin{tabular}{|c|c|c|c|c|c|}
\hline WS id & Waste Stream Name & $\begin{array}{l}\text { Matrix } \\
\text { Code }\end{array}$ & $\begin{array}{l}\text { Inven- } \\
\text { tory }\end{array}$ & $\begin{array}{l}5 \text { yr pro- } \\
\text { jection }\end{array}$ & $\begin{array}{r}\text { Remaining } \\
\text { life-cycle } \\
\text { projection }\end{array}$ \\
\hline RL-W696 & CY95 Hanford MSO CH RCRA/PCE/LLW Unk/oth Debris w/o org w/ met & 55900 & 0.21 & 0.00 & 0.00 \\
\hline RL-W698 & CY95 Hanford MGO CH RCRA/LLW Unk/Oth Matrix w/ org $w /$ met cor & 09999 & 0.62 & 0.00 & 0.00 \\
\hline RL-W699 & CY95 Hanford M\&O CH RCRA/LLW Unk/Oth Matrix w/ org w/o met & U9999 & 32.11 & 0.00 & 0.00 \\
\hline RL-W701 & CY95 Hanford M\&O CH RCRA/LiW Org Lab Packs w/ org w/ met ign & $\mathrm{x} 6100$ & 0.42 & 0.00 & 0.00 \\
\hline RL-W702 & CY95 Hanford M\&O CH RCPA/LLW Org lab Packs w/ org w/ met cor & $\mathrm{x} 6100$ & 0.42 & 0.00 & 0.00 \\
\hline RL-W703 & CY95 Hanford MGO CH RCRA/LLW Org lab Packs $w /$ org w/ met & $x 6100$ & 0.21 & 0.00 & 0.00 \\
\hline RL-W704 & CY95 Hanford M\&O CH RCRA/LLW Org Lab Packs w/ org w/o met ign & $\mathrm{x} 6100$ & 2.08 & 0.00 & 0.00 \\
\hline RL-W705 & CY95 Hanford M\&O CH RCRA/LLW Org Lab Packs w/ org w/o met & $\mathrm{x} 6100$ & 0.21 & 0.00 & 0.00 \\
\hline RL-W731 & CY95 Pac N Nat Lab CH RCRA/LLW org tab Packs w/ org w/ met cor & $\mathrm{x} 6100$ & 0.21 & 0.00 & 0.00 \\
\hline RL-W7 32 & CY95 Pac N Nat Lab CH RCRA/LLW Org Lab Packs w/ org w/ met ign, cor & $\times 6100$ & 0.21 & 0.00 & 0.00 \\
\hline RL-W733 & CY95 PaC N Nat Lab CH RCRA/LLW Org Lab Packs w/ org w/o met ign & $\times 6100$ & 0.42 & 0.00 & 0.00 \\
\hline RL-W734 & CY95 Pac N Nat Lab CH RCRA/LLW Org tab Packs w/ org w/o met cor & $\times 6100$ & 0.83 & 0.00 & 0.00 \\
\hline RL-W735 & CY95 Pac N Nat Lab CH RCRA/LLW Ofg Lab Packs w/ org w/o met & $\times 6100$ & 1.04 & 0.00 & 0.00 \\
\hline RL-w751 & CY $96 \mathrm{CH}$-LLW/RCRA liq,part, soils, labpacks w/org \& thet (thru 6/30) & 53900 & 1.46 & 0.00 & 0.00 \\
\hline RL. $-W 752$ & CY 96 CH-LLW/RCRA liq,part, soils, Labpacks, PCB's w/org thru 6/30) & $\$ 3900$ & 12.58 & 0.00 & 0.00 \\
\hline Thermal & treatment tota 1 & & 750.16 & 479.73 & $1,393.87$ \\
\hline RL-W161 & Radchem Engr Lab Cleaning Material & 53114 & 1.00 & 4.00 & 4.00 \\
\hline RL-W164 & Radchem Engr Lab Liquid Metal Seal & 55110 & 1.00 & 0.00 & 0.00 \\
\hline RL-W165 & Radchem Engr Lab Lead & $\times 7211$ & 1.00 & 0.00 & 0.00 \\
\hline RL-W1 66 & Radchem Engr Lab Oil Absorption Material & 53114 & 1.00 & 0.00 & 0.00 \\
\hline RL-W\$ 74 & Dal-shl tank retrieval MLLW/RH inorg homo solids w/org & $\$ 3190$ & 0.00 & 0.00 & 408.19 \\
\hline $\mathrm{RL}=\mathrm{W} 475$ & Sgl-shl tank retrieval MLLW/RH inorg debris & 55190 & 0.00 & 0.00 & $1,596.89$ \\
\hline RL-W4 77 & Dbl-shl tank retrieval MLLW/RH het debris & 55490 & 0.00 & 0.00 & 100.72 \\
\hline $\mathrm{RL}-\mathrm{W} 480$ & Decontam fac MLLW/RH inorg homo solids & 53190 & 0.00 & 3.02 & 0.00 \\
\hline RL-W481 & Decontam fac MLLW/RH metal debris & 55119 & 0.00 & 15.40 & 0.00 \\
\hline$R L-W 482$ & Decontam fac MLLW/RH org debris & 55390 & 0.00 & 11.20 & 0.00 \\
\hline$R L-W 483$ & Decontam fac MLIW/RH het debris & 55490 & 0.00 & 17.02 & 0.00 \\
\hline RL-W484 & Decontam fac MLLW/RH soils & $\$ 4200$ & 0.00 & 2.52 & 0.00 \\
\hline RL-W485 & Decontam fac MLLW/RH lead & $x 7219$ & 0.00 & 3.30 & 0.00 \\
\hline RL-W498 & Tank long-length equlp MLLW/RH metal debris & $\$ 5119$ & 0.00 & 589.61 & $21,928,35$ \\
\hline RL-W566 & Fuel stab MLLW/RH metal debris & 55119 & 0.00 & 65.80 & 0.00 \\
\hline RL-W567 & Decontam fac MLLW/RH batteries & X7490 & 0.00 & 0.50 & 0.00 \\
\hline$R L-W 568$ & Decontam fac MLLW/RH elemental mercury & $x>100$ & 0.00 & 0.50 & 0.00 \\
\hline$R L+W 569$ & Decontam fac MLLW/RH lab packs & $x 6100$ & 0.00 & 2.52 & 0.00 \\
\hline RL $-\$ 570$ & Inft tank retrieval project MLLW/RH inorg homo soljds w/org & 53190 & 0.00 & 59.91 & $1,269.13$ \\
\hline RL-W571 & Init tank retrieval project MLEW/RH metal debris & $\$ 5119$ & 0.00 & 311,00 & $1,206.72$ \\
\hline RL $-\omega 572$ & Init tank retrieval project MLLW/RH het debris & $\$ 5490$ & 0.00 & 29.63 & 29.63 \\
\hline RL-W573 & Tank farm upgrade project MLIW/RH pred inorg debris & $\$ 5420$ & 0.00 & 81.45 & 88.65 \\
\hline RL-WS74 & Tank farm upgrade project MLLW/RH org debris & 55390 & 0.00 & 61.54 & 66.98 \\
\hline RL-W575 & Tank farm upgrade project MLIW/RH soils & 54200 & 0.00 & 38.01 & 41.37 \\
\hline RL $-W 577$ & Dbl-shl tank retrieval MLLW/RH inorg homo solids w/met & $\$ 3190$ & 0.00 & 0.00 & 45.35 \\
\hline RL-W578 & Init tank retrieval project $M L L W / R H$ inorg homo solids w/met & $\$ 3190$ & 0.00 & 6.66 & 141.01 \\
\hline RL-W716 & CY95 Hanford M\%O RH RCRR/LLW Sandblasting Media w/o org w/ met & $\$ 3112$ & 9.48 & 0.00 & 0.00 \\
\hline RL-W717 & CY95 Hanford Mro RH RCRA/LLW Inorg Debr w/ org w/ met & $\$ 5190$ & 77.62 & 0.00 & 0.00 \\
\hline
\end{tabular}


Table 6. Mixed low-level waste streams by targetted treatment capability (m3) (sheet 10 of 10 ).

\begin{tabular}{|c|c|c|c|c|c|}
\hline WS_id & Waste Stream Name & $\begin{array}{l}\text { Matrix } \\
\text { Code }\end{array}$ & $\begin{array}{l}\text { Inven- } \\
\text { tory }\end{array}$ & $\begin{array}{l}5 \text { yr pro- } \\
\text { jection }\end{array}$ & $\begin{array}{l}\text { Remaining } \\
\text { life-cycle } \\
\text { projection }\end{array}$ \\
\hline$R L-W 718$ & CY95 Hanford M\&O RH RCRA/LLW Composite Filters $w /$ org $w /$ met & 55410 & 6.70 & 0.00 & 0.00 \\
\hline RI-W719 & CY95 Hanford MaO RH RCRA/LLW Pred Inorg debr w/ org w/ met & 55420 & 11.62 & 0.00 & 0.00 \\
\hline$R L-W 720$ & CY95 Hanford MsO RH RCRA/LLW Pred Inorg debr w/ org w/o met & 55420 & 8. 30 & 0.00 & 0.00 \\
\hline RL $-W 721$ & CY95 Hanford MsO RH RCRA/LLW Unk/oth Debris $w /$ org $w /$ met & $\mathbf{5} 5900$ & 8.51 & 0.00 & 0.00 \\
\hline $\mathrm{RL}-\mathrm{W} 742$ & CY95 Pac $\mathrm{N}$ Nat Lab RH RCRA/LLW org Debr $\mathrm{w} /$ org $w /$ met & $\$ 5390$ & 0.21 & 0.00 & 0.00 \\
\hline $\mathrm{RL}-\mathrm{W} 743$ & CY95 Pac $N$ Nat Lab RH RCRA/LIW Pred org Debr w/ org w/ met & 55440 & 0.21 & 0.00 & 0.00 \\
\hline $\mathrm{RL}-\mathrm{W} 744$ & CY95 Pac N Nat Lab RH RCRA/LIW Unk/oth Debris w/o org w/ met & $\$ 5900$ & 5.30 & 0.00 & 0.00 \\
\hline $\mathrm{RL}-\mathrm{W} 745$ & Future routine onsite RH RCRA/LLW Homo Solids & 53900 & 0.00 & 24.30 & 72.54 \\
\hline$R L-W 746$ & Future routine onsite RH RCRA/LLW Metal Debris & 55119 & 0.00 & 44.28 & 209.35 \\
\hline $\mathrm{RL}-\mathrm{W} 747$ & Future routine onsite RH RCRA/LLW Non-fnetal Debris & $\$ 5129$ & 0.00 & 30.82 & 145.69 \\
\hline$R L-W 748$ & Future routine onsite RH RCRA/LLW Org Debris & $\$ 5390$ & 0.00 & 59.99 & 283.63 \\
\hline $\mathrm{RL}-\mathrm{W} 749$ & Future routine onsite RH RCRA/LLW Heter debris & $\$ 5490$ & 0.00 & 1.03 & 4.89 \\
\hline RL-W750 & Future routine onsite RH RCRA/LLW Lead waste & $\times 7290$ & 0.00 & 22.86 & 93.97 \\
\hline Technology & development total & & 131.94 & $1,486.87$ & $27,737.08$ \\
\hline$R L-W 234$ & Knolls Naval Shipyard core basket & 21200 & 21.70 & 0.00 & 0.00 \\
\hline $\mathrm{RL}-\mathrm{W} 756$ & CY $96 \mathrm{CH}$-LLW/RCRA final form macroencapsulated waste (thru $6 / 30$ ) & 21200 & 22.77 & 0.00 & 0.00 \\
\hline$R E-W 75 ?$ & MLLW-INORGANIC DEBRIS-SOLV/TC MET & $\mathrm{Z} 1200$ & 42.00 & 0.00 & 0.00 \\
\hline \multicolumn{2}{|c|}{ Macroencapsulation total } & & 86.47 & 0.00 & 0.00 \\
\hline \multicolumn{2}{|l|}{ Grand Total } & & 8.169 .57 & $4,200.44$ & $55,374.19$ \\
\hline
\end{tabular}




\begin{tabular}{|c|c|}
\hline Matrix code & Physical matrix description \\
\hline L1110 & Acid wastewaters \\
\hline $\mathrm{L} 1220$ & Basic aqueous slurries \\
\hline $\mathbf{L} 2120$ & Aqueous/non-halogenated organic liquids \\
\hline $\mathrm{L} 2200$ & Pure organic liquids \\
\hline L2290 & Non-specified organic liquids \\
\hline$\$ 3110$ & Inorganic particulates \\
\hline S3111 & Ash \\
\hline$\$ 3112$ & Sandblasting media \\
\hline S3113 & Inorganic particulates absorbents \\
\hline$\$ 3114$ & Absorbed organic liquids \\
\hline $\mathbf{S} 3119$ & Non-specified inorganic particulates \\
\hline S3122 & Pond sludges \\
\hline S3129 & Non-specified inorganic sludges \\
\hline S3132 & Paint sludges \\
\hline S3140 w/met & Salt wastes with metals \\
\hline S3140 w/met+org & Salt wastes with metals and organics \\
\hline S3141 & Chloride salts \\
\hline S3142 & Sulfate salts \\
\hline S3143 & Nitrate salts \\
\hline $\mathbf{S} 3149$ & Non-specified salts \\
\hline$\$ 3150$ & Solidified homogeneous solids \\
\hline S3160 & Inorganic chemicals \\
\hline S3190 & Non-specified inorganic homogeneous solids \\
\hline S3190 w/met & Non-specified inorganic homogeneous solids w/met \\
\hline S3190 w/org & Non-specified inorganic homogeneous solids w/ org \\
\hline S3200 & Organic homogeneous solids \\
\hline
\end{tabular}


Table 7. Physical Matrix Code Look-up Table. (3 sheets)

\begin{tabular}{|c|c|}
\hline Matrix code & Physical matrix description \\
\hline S3223 & Non-halogenated organic sludges \\
\hline $\mathbf{S 3 9 0 0}$ & Non-specified homogeneous solids \\
\hline S4100 & Soil \\
\hline $\mathrm{S} 4200$ & Soil/debris \\
\hline S5100 & Inorganic debris \\
\hline$\$ 5110$ & Metal debris \\
\hline S5119 & Non-specified metal debris \\
\hline S5120 & Inorganic non-metal debris \\
\hline S5121 & Concrete debris \\
\hline $\mathbf{S} 5122$ & Glass debris \\
\hline S5125 & Asbestos debris \\
\hline S5129 & Non-specified inorganic non-metal debris \\
\hline S5190 & Non-specified inorganic debris \\
\hline S5300 & Organic debris \\
\hline $\mathbf{S} 5319$ & Non-specified plastic/rubber debris \\
\hline S5340 & Biological debris \\
\hline $\mathbf{S} 5390$ & Non-specified organic debris \\
\hline S5400 & Heterogeneous debris \\
\hline S5410 & Composite filter debris \\
\hline S5420 & Predominantly inorganic debris \\
\hline S5440 & Predominantly organic debris \\
\hline S5490 & Non-specified heterogeneous debris \\
\hline S5900 & Non-specified debris \\
\hline $\mathbf{S} 9000$ & Non-specified solids \\
\hline U9999 & Unknown matrix \\
\hline $\mathrm{X} 6100$ & Organic lab packs \\
\hline
\end{tabular}




\begin{tabular}{|c|c|}
\hline Matrix code & Physical matrix description \\
\hline$\times 6200$ & Inorganic lab packs \\
\hline$\times 6900$ & Non-specified lab packs \\
\hline $\mathrm{X} 7100$ & Elemental mercury \\
\hline $\mathrm{X} 7210$ & Elemental lead \\
\hline $\mathrm{X} 7211$ & Elemental non-activated lead \\
\hline $\mathrm{X} 7219$ & Non-specified elemental lead \\
\hline $\mathrm{X} 7220$ & Elemental cadmium \\
\hline $\mathrm{X} 7290$ & Non-specified elemental hazardous metals \\
\hline X7410 & Lead acid batteries \\
\hline $\mathrm{X} 7420$ & Cadmium batteries \\
\hline $\mathrm{X} 7430$ & Mercury batteries \\
\hline $\mathrm{X} 7490$ & Non-specified batteries \\
\hline $\mathrm{X} 7510$ & Bulk reactive metals \\
\hline X7590 & Non-specified reactive metals \\
\hline $\mathrm{X} 7600$ & Explosives/propellants \\
\hline $\mathrm{Z} 1200$ & Macroencapsulated forms \\
\hline
\end{tabular}

\section{REFERENCES}

RW, 1996, Integrated Data Base Report - 1995: U.S. Spent Nuclear Fuel and Radioactive Waste Inventories, Projections, and Characteristics, DOE/RW-0006, Rev. 12, U.S. Department of Energy, Washington, D.C. 
DOE/RL-97-14

This page intentionally left blank. 


\section{DISTRIBUTION}

\section{Number of Copies}

\section{OFFSITE}

Washington State Department

of Ecology

Kennewick, Washington 99336

$\begin{array}{ll}\text { M. N. Jaraysi (2) B5-18 } & \text { B }\end{array}$

U.S. Environmental Protection Agency 1200 Sixth Ave.

Seattle, Washington 98101

D. Ingemansen

D. Bartus

U.S. Environmental Protection Agency

Richland. Washington 99352

D. R. Sherwood (2) B5-01

U.S. Department of Energy-Headquarters Washington, D.C.
R. A. Martinez
EM-38
P. Bubar
EM-35

\section{Nez Perce Tribe}

Environmental Restoration and

Waste Management Program

P.O. Box 365

Lapwai, Idaho 83540

D. Powaukee, Manager

Confederated Tribes of the Umatilla Indian Reservation Board of Trustees

P.O. Box 638

Pendleton, Oregon 97801

J. R. Wilkinson 


\title{
DISTRIBUTION (cont)
}

\author{
Yakama Indian Nation \\ Environmental Restoration and \\ Waste Management Program \\ P.O. Box 151 \\ Toppenish, Washington 98948 \\ R. Jim, Manager
}

\section{Number of Copies}

ONSITE

14

U.S. Department of Energy, Richland Operations Office

4
K. D. Bazzell
S7-55
R. F. Guercia (11)
S7-55
J. E. Kinzer
S7-50
Public Reading Room
$\mathrm{H} 2-53$

Bechtel Hanford.Incorporated

J. W. Badden

H9-11

R. J. Landon

$\mathrm{H} 0-10$

L. A. Mihalik

H9-01

G. S. Robinson

$\mathrm{H} 0-18$

Pacific Northwest National Laboratory

B. J. Day

P7-28

E. A. Flores

P7-79

M. W. McCoy

P7-79

W. A. Ross

K7-94

M. H. Schlender

K9-18

H. T. Tilden II

P7-79

Technical Files

K1-11 


\section{DISTRIBUTION (cont)}

$\begin{array}{ll}\text { D. Alison } & \text { R1-51 } \\ \text { T. L. Baker (5) } & \text { H6-06 } \\ \text { T. G. Beam } & \text { S4-66 } \\ \text { D. G. Black (25) } & \text { H6-20 } \\ \text { W. R. Brown } & \text { B2-35 } \\ \text { D. J. Carrell } & \text { R1-51 } \\ \text { F. M. Coony } & \text { H6-06 } \\ \text { T. A. Dillhoff } & \text { N2-57 } \\ \text { R. H. Engelmann } & \text { H6-26 } \\ \text { B. G. Erlandson } & \text { R2-36 } \\ \text { D. L. Flyckt } & \text { S6-71 } \\ \text { G. D. Hendricks } & \text { R3-56 } \\ \text { W. S. Josephson } & \text { H6-06 } \\ \text { S. E. Killoy } & \text { S4-66 } \\ \text { O. S. Kramer } & \text { B2-35 } \\ \text { G. J. LeBaron } & \text { S6-19 } \\ \text { S. S. Lowe } & \text { H6-06 } \\ \text { E. E. Mayer } & \text { R2-36 } \\ \text { D. E. McKenney } & \text { H6-06 } \\ \text { A. G. Miskho } & \text { H6-22 } \\ \text { D. E. Nester } & \text { H6-06 } \\ \text { D. H. Nichols } & \text { H6-06 } \\ \text { P. A. Powell (2) } & \text { R1-51 } \\ \text { J. O. Skolrud } & \text { H6-20 } \\ \text { J. N. Strode } & \text { R2-11 } \\ \text { C. N. Villalobos } & \text { S6-19 } \\ \text { P. J. Weaver } & \text { L1-02 } \\ \text { A. G. Weiner } & \text { H6-21 } \\ \text { B. D. Williamson } & \text { B3-15 } \\ \text { M. T. Yasdick } & \text { H6-10 } \\ \text { EPIC (7) } & \text { H6-08 } \\ \text { Central Files } & \text { A3-88 } \\ \text { DPC } & \text { A3-94 }\end{array}$




\section{DOE/RL-97-14}

This page intentionally left blank.

Distr-4 